\title{
TENSOR-BASED TIME-DELAY ESTIMATION TECHNIQUES FOR THIRD GENERATION GLOBAL POSITIONING SYSTEM
}

MATEUS DA ROSA ZANATTA

DISSERTAÇÃO DE MESTRADO EM SISTEMAS MECATRÔNICOS DEPARTAMENTO DE ENGENHARIA MECÂNICA 
UNIVERSIDADE DE BRASÍLIA

FACULDADE DE TECNOLOGIA

DEPARTAMENTO DE ENGENHARIA MECÂNICA

\section{TENSOR-BASED TIME-DELAY ESTIMATION TECHNIQUES FOR THIRD GENERATION GLOBAL POSITIONING SYSTEM}

\section{MATEUS DA ROSA ZANATTA}

Orientador: Prof. Dr.-Ing. João Paulo C. L. da Costa, ENE/UnB

Coorientador: Dr.-Ing Ricardo Kehrle Miranda, PPGEE/UnB

DISSERTAÇÃO DE MESTRADO EM SISTEMAS MECATRÔNICOS

PUBLICAÇÃO PPMEC.DM - 138/2018

BRASÍLIA-DF, 01 DE FEVEREIRO DE 2018. 


\author{
UNIVERSIDADE DE BRASÍLIA \\ FACULDADE DE TECNOLOGIA \\ DEPARTAMENTO DE ENGENHARIA MECÂNICA
}

\title{
TENSOR-BASED TIME-DELAY ESTIMATION TECHNIQUES FOR THIRD GENERATION GLOBAL POSITIONING SYSTEM
}

\section{MATEUS DA ROSA ZANATTA}

DISSERTAÇÃO DE MESTRADO ACADÊMICO SUBMETIDA AO DEPARTAMENTO DE ENGENHARIA MECÂNICA DA FACULDADE DE TECNOLOGIA DA UNIVERSIDADE DE BRASÍLIA, COMO PARTE DOS REQUISITOS NECESSÁRIOS PARA A OBTENÇÃO DO GRAU DE MESTRE EM SISTEMAS MECATRÔNICOS.

APROVADA POR: Prof. Dr.-Ing. João Paulo C. L. da Costa, ENE/UnB
Orientador

Prof. Dr. Ricardo Zelenovsky, ENE/UnB

Examinador interno

Prof. Dr.-Ing. Felix Antreich, DETI/UFC

Examinador externo

BRASÍLIA, 01 DE FEVEREIRO DE 2018. 


\section{FICHA CATALOGRÁFICA}

MATEUS DA ROSA ZANATTA

Tensor-Based Time-Delay Estimation Technique for Third Generation Global Positioning System

2018xv, 131p., 201x297 mm

(ENM/FT/UnB, Mestre, Sistemas Mecatrônicos, 2018)

Dissertação de Mestrado - Universidade de Brasília

Faculdade de Tecnologia - Departamento de Engenharia Mecânica

\section{REFERÊNCIA BIBLIOGRÁFICA}

MATEUS DA ROSA ZANATTA (2018) Tensor-Based Time-Delay Estimation Technique for Third Generation Global Positioning System. Dissertação de Mestrado em Sistemas Mecatrônicos, Publicação 138/2018, Departamento de Engenharia Mecânica, Universidade de Brasília, Brasília, DF, 131p.

\section{CESSÃO DE DIREITOS}

AUTOR: Mateus da Rosa Zanatta

TÍTULO: Tensor-Based Time-Delay Estimation Technique for Third Generation Global Positioning System.

GRAU: Mestre ANO: 2018

É concedida à Universidade de Brasília permissão para reproduzir cópias desta dissertação de Mestrado e para emprestar ou vender tais cópias somente para propósitos acadêmicos e científicos. Ao autor se reserva outros direitos de publicação e nenhuma parte desta dissertação de Mestrado pode ser reproduzida sem a autorização por escrito do autor.

Mateus da Rosa Zanatta

CLN 212 BL B APTO 208 


\section{Agradecimentos}

Gostaria de primeiramente agradecer à meus pais pelo apoio e conselhos dados durante o decorrer deste mestrado. Também, agradecer meu orientador de Mestrado, Dr.-Ing. João Paulo Lustosa, por sua liderança e orientação, além das oportunidades que este me proporcionou ao me indicar como aluno visitante na TU Ilmenau.

Também gostaria de agradecer meu coorientador de Mestrado, Dr.-Ing Ricardo Kehrle Miranda, que dedicou seu tempo à acompanhar meu desenvolvimento como pesquisador juntamente à meu orientador. 


\section{Dedicatória}

Aos meus pais Mario e Luciane, que dignamente me apresentaram à importância da família e ao caminho da honestidade e persistência. 


\section{Abstract}

Global Navigation Satellite Systems (GNSS), such as American GPS, European Galileo, Russian GLONASS, and Chinese BeiDou, are crucial for applications ranging from traditional civilian aviation to missile guiding. Furthermore, the number of GNSS applications have been increasing, for instance, fishing authorities can use GNSS to monitor fishing ships and guarantee the sustainable management of its fishing areas. In addition, GNSS can be utilized for automatic toll system and for autonomous vehicle. Finally, GNSS can be used in precision farming for increasing precision of fertilization and allow the utilization of agriculture vehicles 24 hour a day. Such applications require a very accurate positioning even in complex scenarios, where multipath components are present. Thus, in order to introduce a better separation between transmitted signals, the third generation of GPS adds the L1 civil (L1C) pilot code to operate alongside the standard Coarse Acquisition (C/A). Moreover, the L1C pilot code includes a Time Multiplexed Binary Offset Carrier (TMBOC) in order to have a better performance in multipath scenarios and provide a better spectral separation of the signal which limits intra-system and inter-system interference.

In this thesis, we study the state-of-the-art Higher-Order Singular Value Decomposition (HOSVD) based method to time-delay estimation, and the state-of-the-art Canonical Polyadic Decomposition by Generalized Eigenvalue Decomposition (CPD-GEVD) based timedelay estimation approach. Furthermore, we show that both state-of-the-art approaches can be successfully applied to the third generation GPS L1C pilot code. In addition, we propose a Semi-algebraic Framework for Approximate Canonical Polyadic Decomposition via Simultaneous Matrix Diagonalization (SECSI) with HOSVD based time-delay estimation scheme for second and third generation global positioning system. The SECSI based approach inherits all the advantages of the CPD-GEVD and can be also combined with the L1C signaling and TMBOC modulation. 


\section{CONTENTS}

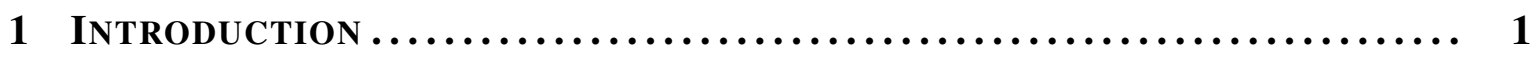

2 Data Model $\ldots \ldots \ldots \ldots \ldots \ldots \ldots \ldots \ldots \ldots \ldots \ldots \ldots \ldots \ldots \ldots \ldots \ldots, \quad 3$

2.1 DATA MODEL SCENARIO ................................... 4

2.2 L1C SIGNAL GENERATION .................................... 5

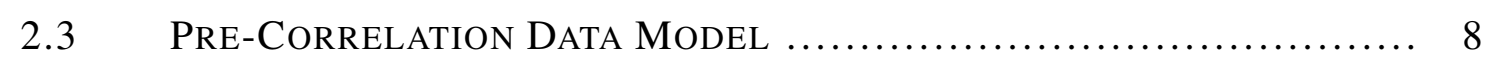

2.4 Post-Correlation Data Model ............................... 9

3 Tensor-Based Time-Delay Estimation and Simulations .......... 10 3.1 State-OF-The-Art Tensor-Based Time-Delay Estimation

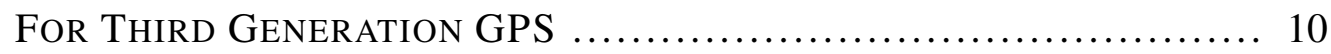

3.1.1 HOSVD BASED TIME-DELAY ESTIMATION ........................ 11

3.1.2 DoA/KrF BASEd TIME-DELAy Estimation ....................... 12

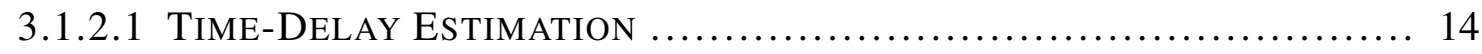

3.1.3 CPD-GEVD BASED TIME-DELAy ESTIMATION...................... 15

3.2 Proposed Semi-Algebraic Framework FOR Approximate Canonical Polyadic Decomposition via Simultaneous MaTRIX Diagonalization BASED FRAMEWORK FOR TIME-DELAY Es-

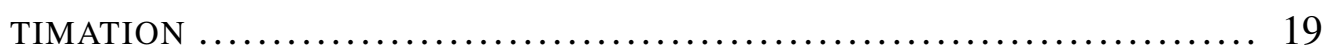

3.3 COMPUTATIONAL COMPLEXITY ................................ 24

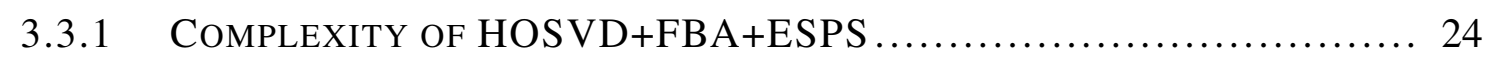

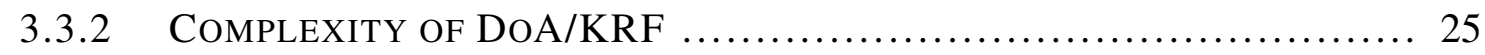

3.3.3 COMPLEXITY OF CPD-GEVD ................................. 26

3.3.4 COMPLEXITY OF HOSVD SECSI ............................. 26

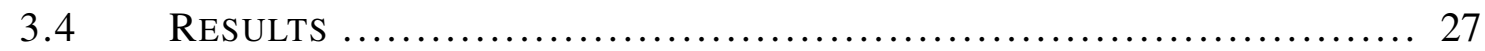

3.4.1 StATE-OF-THE-ART SCEnARIO ................................ 27

3.4.2 PROPOSED SCENARIO .......................................... 29

3.4.2.1 SECOND Generation GPS Time-Delay Estimation ................ 30

3.4.2.2 Third Generation GPS Time-Delay Estimation.................. 37

3.4.2.3 ANTENNA ARRAY WITH ERRORS ................................ 46

3.4.2.4 RECEIVEd Signals With VARYing DoA ......................... 49

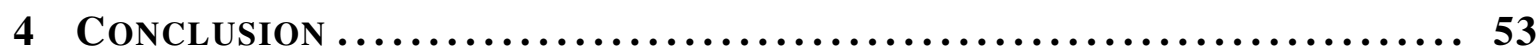


4.1 FUTURE WORKS

BIBLIOGRAPHY $\ldots \ldots \ldots \ldots \ldots \ldots \ldots \ldots \ldots \ldots \ldots \ldots \ldots \ldots \ldots \ldots \ldots \ldots \ldots \ldots \ldots$

Appendix a Tensor Calculus................................ 59

A.1 Notation ....................................................... 59

A.2 Matrix Calculus .............................................. 60

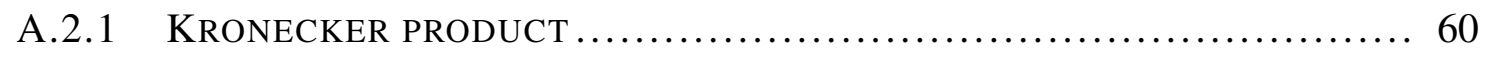

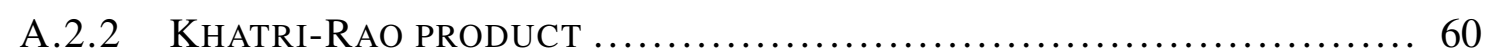

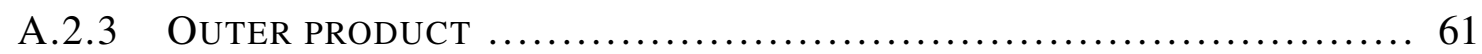

A.2.4 THE vec $\{\cdot\}$ OPERATOR..................................... 61

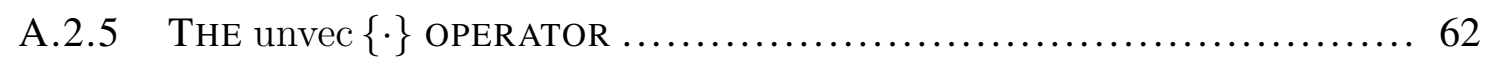

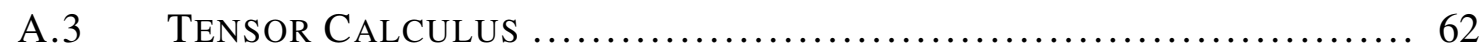

A.3.1 TENSORS .................................................. 62

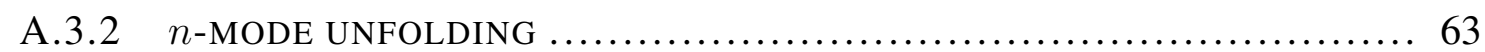

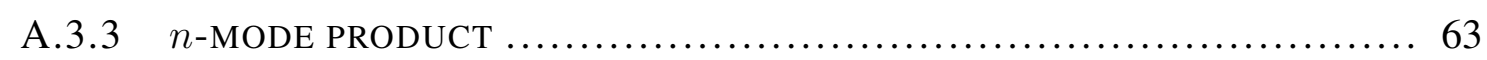

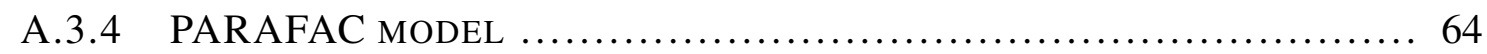

A.3.5 HIGHER-ORDER SVD ............................................. 64

A.3.6 DUAL-SYMMETRIC TENSORS ................................. 65

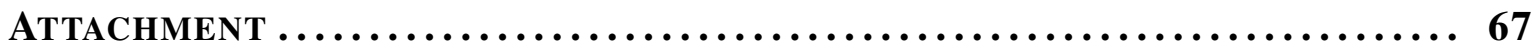

A The Double-Arc Positioner $\ldots \ldots \ldots \ldots \ldots \ldots \ldots \ldots \ldots \ldots \ldots \ldots \ldots \ldots$

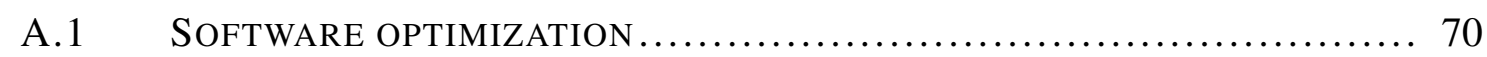

B IntRACRANIAL SignALS $\ldots \ldots \ldots \ldots \ldots \ldots \ldots \ldots \ldots \ldots \ldots \ldots \ldots \ldots \ldots \ldots \ldots \ldots$

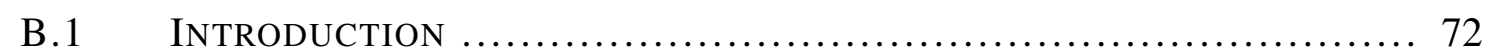

B.2 BiOLOGICAL CONCEPTS ........................................ 73

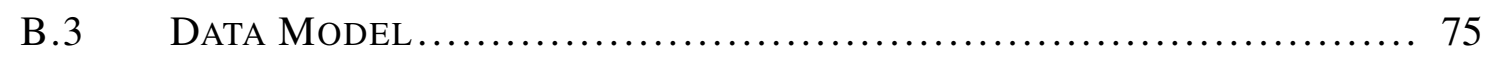

B.4 Time-Scale RePresentation ................................ 76

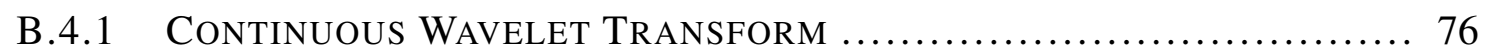

B.5 Proposed technique...................................... 76

B.6 RESULTS .......................................................... 77

B.7 COnClusion ............................................... 81

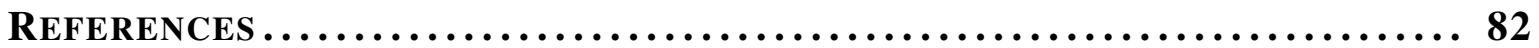




\section{Resumo $^{1}$}

Sistemas de satélites de navegação global, do inglês Global Navigation Satellite System (GNSS), como o GPS Americano, Galileo, GNSS Europeu, GLONASS, GNSS Russo, e o BeiDou, GNSS Chinês, são de extrema importância para aplicações que vão desde a aviação civil à mísseis guiados. Ademais, o número de de aplicações que utilizam GNSS têm crescido consideravelmente, por exemplo, autoridades de pesca podem utilizar GNSS para monitorar barcos pesqueiros e garantir um manejo sustentável às áreas de pesca [1]. Além disso, GNSS pode ser utilizado em sistemas de pedágios automáticos [2] e veículos autônomos [3]. Afinal, GNSS pode ser utilizado em agricultura de precisão para aumentar a precisão na fertilização e permitir o uso de máquinas agrícolas 24 horas por dia [4]. Estas aplicações descritas requerem alta precisão no posicionamento do usuário, mesmo em ambientes complexos onde componentes de multipercurso estão presentes. Por isso, para que se tenha uma melhor separação entre sinais transmitidos, o GPS de terceira geração adicionará o sinal L1 civil (LIC) pilot code para ser transmitido ao lado do padrão Coarse Acquisition (C/A). Adicionalmente, LIC pilot code incluirá a Time Multiplexed Binary Offest Carrier (TMBOC) para que se tenha um melhor desempenho em ambientes com componentes de multipercurso e prover uma melhor separação espectral do sinal para diminuir interferências inter-sistema e intra-sistema [5].

Nesta dissertação estudamos o estado-da-arte do método de estimação de atraso baseado na decomposição de valores singulares de alta ordem, do inglês Higher-Order Singular Value Decomposition (HOSVD), o estado-da-arte do método direção de chegada através da fatorização Khatri-Rao, do inglês Direction of Arrival via Khatri-Rao Factorization(DoA/KRF), e o estado-da-arte do método de estimação de atraso baseado na decomposição poliádica canônica por decomposição de autovalores generalizados, do inglês Canonical Polyadic Decomposition by Generalized Eigenvalue Decomposition (CPD-GEVD). Além disso, mostramos que ambos métodos do estado-da-arte podem ser combinados com o sinal L1C pilot code do GPS de terceira geração. Adicionalmente, propomos a utilização da Estrutura Semi-Algébrica para Decomposição Canonical Polyadic Aproximadas Através de Diagonalizações Simultâneas para Estimação de Atraso, do inglês Semi-algebraic framework for

\footnotetext{
${ }^{1}$ Este resumo visa traduzir o resumo em inglês desta dissertação, bem como, apresentar uma versão traduzida e resumida do conteúdo deste documento. Neste capítulo, apresenta-se a tradução resumida do modelo de dados utilizado, da revisão do estado-da-arte, do método proposto e dos resultados obtidos com a realiação das simulações.
} 
approximate Canonical Polyadic Decompositions via Simultaneous Matrix Diagonalizations (SECSI), com HOSVD para realizar a estimação de atraso para GPS de segunda e terceira geração. O método SECSI herda todas as vantagens do CPD-GEVD e pode ser combinado com o sinal L1C e a modulação TMBOC.

\section{Modelo de dados}

Da mesma forma que [6], o modelo de dados considera um arranjo de antenas para GPS de terceira geração, onde supõe-se que $D$ satélites possuam linha de visada, do inglês lineof-sight (LOS), com o receptor e que o número de componentes de multipercurso $L$ é dado por $L=\sum_{d=1}^{D} L_{d}$. Cada satélite transmite $N$ amostras do L1C pilot code modulado pela TMBOC, onde nos referimos à cada janela de tempo como período. Assim, supomos que, durante $K$ períodos, as respectivas $N$ amostras são aproximadamente constantes.

$$
\mathcal{X}=\mathcal{I}_{3, L} \times{ }_{1} \tilde{\boldsymbol{\Gamma}}^{\mathrm{T}} \times{ }_{2} \tilde{\mathbf{C}}^{\mathrm{T}} \times{ }_{3} \tilde{\mathbf{A}}+\mathcal{N}
$$

onde, $\tilde{\boldsymbol{\Gamma}}^{\mathrm{T}}=\left[\tilde{\gamma}_{1}, \ldots,{\tilde{\gamma_{L}}}\right] \in \mathbb{C}^{K \times L}$ é a matriz que contém as amplitudes complexas dos sinais, $\tilde{\mathbf{C}}=\left[\tilde{\mathbf{c}}\left[\tau_{1}\right], \ldots, \tilde{\mathbf{c}}\left[\tau_{L}\right]\right] \in \mathbb{R}^{N \times L}$ concatena em suas linhas o código pseudo-aleatório do L1C pilot code modulado pela TMBOC e $\tilde{\mathbf{A}}=\left[\tilde{\mathbf{a}}\left(\phi_{1}\right), \ldots, \tilde{\mathbf{a}}\left(\phi_{L}\right)\right] \in \mathbb{C}^{M \times L}$ concatena em suas colunas o vetor de direção dos $L$ sinais.

Para que se possa separar o sinal de cada satélite, um banco de correlatores $\mathrm{Q}=\mathrm{Q}_{\omega} \boldsymbol{\Sigma} \mathbf{V}^{\mathrm{H}}$ é gerado para cada satélite. Utilizando-se do banco de correlatores, [7] mostra que o tensor recebido $\mathcal{X} \in \mathbb{C}^{K \times N \times M}$ pode ser correlacionado com o subespaço à esquerda do banco de correlatores $\mathbf{Q}_{\omega}=\mathrm{Q}\left(\boldsymbol{\Sigma} \mathbf{V}^{\mathrm{H}}\right)^{-1}$ [8] obtido ao aplicar a decomposição de valores singulares econômica à $\mathbf{Q}$. Dessa forma temos o seguinte modelo para calcular a correlação cruzada utilizada para estimação de atraso

$$
\begin{aligned}
\mathcal{Y} & =\mathcal{X} \times{ }_{2} \mathbf{Q}_{\omega}{ }^{\mathrm{T}} \\
& =\mathcal{I}_{3, L} \times{ }_{1} \Gamma^{\mathrm{T}} \times{ }_{2}\left(\mathbf{C Q}_{\omega}\right)^{\mathrm{T}} \times{ }_{3} \mathbf{A}+\mathcal{N}_{\omega} .
\end{aligned}
$$

\section{Estado-da-arte para Estimação de atraso}

As técnicas de estado-da-arte para decomposição tensorial estudadas neste trabalho são a decomposição de valores singulares de alta ordem, do inglês Higher-Order Singular Value Decomposition (HOSVD) [7], que se utiliza de técnicas de pré-processamento; o segundo método utilizado neste estudo é direção de chegada através da fatorização Khatri-Rao, do inglês Direction of Arrival via Khatri-Rao Factorization(DoA/KRF); a terceira técnica utilizada para estudo é a decomposição poliádica canônica por decomposição de autovalores generalizados, do inglês Canonical Polyadic Decomposition by Generalized Eigenvalue De- 
composition (CPD-GEVD) [9].

\section{Estimação de atraso utilizando HOSVD}

A estimação de atraso utilizando a decomposição de valores singulares de alta ordem, do inglês Higher-Order Singular Value Decomposition (HOSVD), primeiramente aplica-se a técnica de pré-processamento de média frente costas, do inglês Forward-Backward Averaging (FBA) [10], [11]

$$
\mathbf{Z}=\left[\begin{array}{llll}
{[\mathcal{Y}]_{3}} & \Pi_{M} & {[\mathcal{Y}]_{3}^{*}} & \Pi_{K Q}
\end{array}\right] \in \mathbb{C}^{M \times 2 K Q}
$$

após isso, aplica-se a suavização espacial expandida, do inglês Expanded Spatial Smoothing (ESPS) [12] e [13], em que o arranjo é dividido em $L_{S}$ sub-arranjos com $M_{S}=M-L_{S}+1$ elementos. Para isso, criasse uma matriz de seleção

$$
\mathbf{J}_{\ell_{S}}=\left[\begin{array}{lll}
0_{M_{S} \times \ell_{S}-1} & \mathbf{I}_{M_{S}} & 0_{M_{S} \times L_{S}-1}
\end{array}\right] \in \mathbb{R}^{M_{S} \times M},
$$

para $\ell_{S}=1, \ldots, L_{S}$. Com isso, ESPS é aplicado à (4)

$$
\mathbf{W}=\left[\begin{array}{lll}
\mathbf{J}_{1} \mathbf{Z} & \cdots & \mathbf{J}_{L_{S}} \mathbf{Z}
\end{array}\right] \in \mathbb{C}^{M_{S} \times 2 L_{S} K Q}
$$

em seguida (5) é dobrado como um tensor de quarta ordem $\mathcal{Z}_{E S P S} \in \mathbb{C}^{2 K \times Q \times M_{S} \times L_{S}}$. Após isso HOSVD é aplicado à $\mathcal{Z}_{E S P S}$

$$
\mathcal{Z}_{E S P S}=\mathcal{R} \times{ }_{1} \mathbf{U}_{1} \times{ }_{2} \mathbf{U}_{2} \times_{3} \mathbf{U}_{3} \times_{4} \mathbf{U}_{4}
$$

em que $\mathcal{R} \in \mathbb{C}^{2 K \times Q \times M_{S} \times L_{S}}$ é o tensor núcleo, $\mathbf{U}_{1} \in \mathbb{C}^{2 K \times 2 K}, \mathbf{U}_{2} \in \mathbb{C}^{Q \times Q}, \mathbf{U}_{3} \in \mathbb{C}^{M_{S} \times M_{S}}$, e $\mathbf{U}_{4} \in \mathbb{C}^{L_{S} \times L_{S}}$ são as matrizes singulares que contêm os vetores singulares contendo os desdobramentos de (2).

Uma vez que supõe-se que o componente LOS possui a maior potência, os vetores singulares dominantes são multiplicados por $\mathcal{Z}_{E S P S}$. Após, o vetor resultante é multiplicado por $\Sigma \mathrm{V}^{\mathrm{H}}$ obtidos após aplicar a decomposição de valores singulares econômica à Q. Então, resultando no vetor $\mathbf{q}_{E S P S}$

$$
\mathbf{q}_{E S P S}=\left[\mathcal{Z}_{E S P S} \times_{1}\left(\mathbf{u}_{1}^{(1)}\right)^{\mathrm{H}} \times_{3}\left(\mathbf{u}_{1}^{(3)}\right)^{\mathrm{H}} \times_{4}\left(\mathbf{u}_{1}^{(4)}\right)^{\mathrm{H}}\right] \boldsymbol{\Sigma} \mathbf{V}^{\mathrm{H}}
$$

onde $\mathbf{q}_{E S P S}$ contém os valores de correlação cruzada que foram multidimensionalmente filtrados. Ao vetor resultante $\mathbf{q}_{E S P S}$ é então aplicada a interpolação de spline cúbico para maximizar a resolução da estimação de atraso. 


\section{Estimação de atraso utilizando DoA/KRF}

Em [14] é proposta uma abordagem de três etapas baseada na estimação de direção de chegada, do inglês direction of arrival estimation (DoA), Fatorização Khatri-Rao, do inglês Khatri-Rao factorization (KRF) e a seleção do componente LOS estimado. O processamento para estimação de DoA utiliza a técnica de estimação de parâmetros de sinais através de invariância rotacional, do inglês Estimation of Signal Parameter via Rotational Invariance Technique (ESPRIT) [15], é aplicada à W para estimar $\hat{\mathbf{A}}$.

Após, reescrever (2) para obter a seguinte equação

$$
[\mathcal{Y}]_{(3)}=\mathbf{A}\left[\Gamma^{\mathrm{T}} \diamond\left(\mathbf{C Q}_{\omega}\right)^{\mathrm{T}}\right]^{\mathrm{T}} \in \mathbb{C}^{M \times K Q}
$$

Uma vez que $\hat{\mathbf{A}}$ foi estimado pela técnica ESPRIT, sua pseudo-inversa pode ser aplicada à (8) de tal forma que

$$
\begin{aligned}
\hat{\mathbf{A}}^{+}[\mathcal{Y}]_{3} & =\hat{\mathbf{A}}^{+} \mathbf{A}\left[\boldsymbol{\Gamma}^{\mathrm{T}} \diamond\left(\mathbf{C} \mathbf{Q}_{\omega}\right)^{\mathrm{T}}\right]^{\mathrm{T}} \\
& \approx\left[\boldsymbol{\Gamma}^{\mathrm{T}} \diamond\left(\mathbf{C Q}_{\omega}\right)^{\mathrm{T}}\right]^{\mathrm{T}} \in \mathbb{C}^{L_{d} \times K Q},
\end{aligned}
$$

onde as matrizes de fatores $\boldsymbol{\Gamma}$ e $\left(\mathbf{C Q}_{\omega}\right)$ podem ser estimada por meio da Fatorização KhatriRao de mínimos quadrado, do inglês Least Square Khatri-Rao factorization (LSKRF) [16, 17].

Logo que $\left(\Gamma^{\mathrm{T}} \diamond\left(\mathbf{C Q}_{\omega}\right)^{\mathrm{T}}\right)^{\mathrm{T}}$ é dado em (9), e considerando que sua $\ell_{d}$-ésima coluna pode ser calculada como um produto Khatri-Rao da $\ell_{d}$-ésima coluna de $\Gamma^{\mathrm{T}}$ e $\left(\mathbf{C Q}_{\omega}\right)^{\mathrm{T}}$ :

$$
\left[\boldsymbol{\Gamma}^{\mathrm{T}} \diamond\left(\mathbf{C Q}_{\omega}\right)^{\mathrm{T}}\right]_{\cdot, \ell_{d}}=\left(\boldsymbol{\Gamma}^{\mathrm{T}}\right)_{\cdot, \ell_{d}} \diamond\left(\mathbf{C Q}_{\omega}\right)_{\cdot, \ell_{d}}
$$

onde cada coluna de $\left(\boldsymbol{\Gamma}^{\mathrm{T}} \diamond\left(\mathrm{CQ}_{\omega}\right)^{\mathrm{T}}\right)_{\cdot, \ell_{d}} \in \mathbb{C}^{K Q}$. Assim, para obter as estimativas de $\boldsymbol{\Gamma} \mathrm{e}$ $\mathrm{CQ}_{\omega}$, remodela-se (10) em um matriz de tamanho $Q \times K$

$$
\underset{Q \times K}{\operatorname{unvec}}\left\{\left[\boldsymbol{\Gamma}^{\mathrm{T}} \diamond\left(\mathbf{C Q}_{\omega}\right)^{\mathrm{T}}\right]_{\cdot, \ell_{d}}\right\}=\left(\mathbf{C Q}_{\omega}\right)_{\cdot, \ell_{d}}^{\mathrm{T}}\left(\boldsymbol{\Gamma}^{\mathrm{T}}\right)_{\cdot, \ell_{d}}^{\mathrm{T}}
$$

Uma vez que (11) é uma matriz de posto unitário, pode-se executar o SVD de

$$
\underset{Q \times K}{\operatorname{unvec}}\left\{\left[\boldsymbol{\Gamma}^{\mathrm{T}} \diamond\left(\mathbf{C Q}_{\omega}\right)^{\mathrm{T}}\right]_{, \ell_{d}}\right\}=\mathbf{U}_{\ell_{d}} \boldsymbol{\Sigma}_{\ell_{d}} \mathbf{V}_{\ell_{d}} .
$$

Portando, as estimativas para $\left(\boldsymbol{\Gamma}^{\mathrm{T}}\right)_{\cdot, \ell_{d}} \mathrm{e}\left(\left(\mathbf{C Q}_{\omega}\right)^{\mathrm{T}}\right)_{\cdot, \ell_{d}}$ são $\sqrt{\sigma_{\ell_{d}, 1}} \mathbf{v}_{\ell_{d}, 1}^{*} \mathrm{e} \sqrt{\sigma_{\ell_{d}, 1}} \mathbf{u}_{\ell_{d}, 1}$, res- 
pectivamente, onde $\sigma_{\ell_{d}, 1}$ é o vetor singular dominante de $\Sigma_{\ell_{d}}$, $\mathbf{v}_{\ell_{d}, 1}^{*}$ é o conjugado do vetor singular dominante à direita de $\mathbf{V}_{\ell_{d}}$ e $\mathbf{u}_{\ell_{d}, 1}$ o vetor singular dominante à esquerda de $\mathbf{U}_{\ell_{d}}$. Isto é repetido para $\ell_{d}=1, \ldots, L_{d}$.

\section{Estimação de atraso utilizando CPD-GEVD}

Outra técnica tensorial a ser utilizada é a decomposição poliádica canônica por decomposição de autovalores generalizados, do inglês Canonical Polyadic Decomposition by Generalized Eigenvalue Decomposition (CPD-GEVD). CPD-GEVD primeiramente aplica o HOSVD à $\mathcal{Y}$ a partir de (2)

$$
\mathcal{Y} \approx \mathcal{S}^{\mathrm{g}} \times{ }_{1} \mathrm{U}_{1}^{\mathrm{g}} \times{ }_{1} \mathrm{U}_{2}^{\mathrm{g}} \times{ }_{3} \mathrm{U}_{3}^{\mathrm{g}}
$$

em que $\mathbf{U}_{1}^{\mathrm{g}} \in \mathbb{C}^{K \times L_{d}}, \mathbf{U}_{2}^{\mathrm{g}} \in \mathbb{C}^{Q \times L_{d}}$, e $\mathbf{U}_{3}^{\mathrm{g}} \in \mathbb{C}^{M \times L_{d}}$, e $\mathcal{S}^{\mathrm{g}} \in \mathbb{C}^{L_{d} \times L_{d} \times L_{d}}$ é o tensor núcleo que pode ser expressado como uma decomposição PARAFAC:

$$
\mathcal{S}^{\mathrm{g}}=\mathcal{I}_{3, L} \times_{1} \mathbf{T}_{1}^{\mathrm{g}} \times_{2} \mathbf{T}_{2}^{\mathrm{g}} \times_{3} \mathbf{T}_{3}^{\mathrm{g}}
$$

onde as primeiras duas fatias frontais de $\mathcal{S}^{\mathrm{g}}$ podem ser escritas como:

$$
\begin{aligned}
& \left(\mathcal{S}^{\mathrm{g}}\right)_{\cdot, \cdot, 1}=\mathbf{T}_{1}^{\mathrm{g}} \operatorname{diag}\left\{\left(\mathbf{T}_{3}^{\mathrm{g}}\right)_{\cdot, 1}\right\}\left(\mathbf{T}_{2}^{\mathrm{g}}\right)^{\mathrm{T}} \\
& \left(\mathcal{S}^{\mathrm{g}}\right)_{\cdot, \cdot, 2}=\mathbf{T}_{1}^{\mathrm{g}} \operatorname{diag}\left\{\left(\mathbf{T}_{3}^{\mathrm{g}}\right)_{\cdot, 2}\right\}\left(\mathbf{T}_{2}^{\mathrm{g}}\right)^{\mathrm{T}} .
\end{aligned}
$$

Assim, o GEVD é calculado à partir das matrizes $\left(\mathcal{S}^{\mathrm{g}}\right)_{\cdot, \cdot, 1}$ e $\left(\mathcal{S}^{\mathrm{g}}\right)_{\cdot,, 2}$ :

$$
\left(\mathcal{S}^{\mathrm{g}}\right)_{\cdot, \cdot, 1}^{\mathrm{T}} \mathbf{E}=\left(\mathcal{S}^{\mathrm{g}}\right)_{\cdot, \cdot, 2}^{\mathrm{T}} \mathbf{E D}
$$

onde $\mathbf{D}$ contém os autovalores em sua diagonal, e E contêm os autovetores. Nota-se que (16) é equivalente à

$$
\begin{aligned}
\left(\mathcal{S}^{\mathrm{g}}\right)_{\cdot, \cdot, 2}^{-\mathrm{T}}\left(\mathcal{S}^{\mathrm{g}}\right)_{\cdot, \cdot, 1}^{\mathrm{T}} & =\mathbf{E D E}^{-1} \\
& =\left(\mathbf{T}_{1}^{\mathrm{g}}\right)^{-\mathrm{T}} \operatorname{diag}\left\{\left(\mathbf{T}_{3}^{\mathrm{g}}\right)_{\cdot, 2}\right\}^{-1} \operatorname{diag}\left\{\left(\mathbf{T}_{3}^{\mathrm{g}}\right)_{\cdot, 1}\right\} \mathbf{T}_{1}^{\mathrm{g}}
\end{aligned}
$$

Dessa forma, a Equação (17) é um problema de diagonalização em que $\mathbf{E}$ fornece uma estimativa de $\left(\mathbf{T}_{1}^{\mathrm{g}}\right)^{-\mathrm{T}}$. Assim, ao combinar $\left(\mathbf{T}_{1}^{\mathrm{g}}\right)^{-\mathrm{T}}$ de (17) e $\mathbf{U}_{1}^{*}$ de (13) a matriz de fatores $\Gamma^{-\mathrm{T}}$ pode ser estimada como

$$
\hat{\boldsymbol{\Gamma}}^{+\mathrm{T}}=\left(\mathbf{U}_{1}^{\mathrm{g}}\right)^{*}\left(\mathbf{T}_{1}^{\mathrm{g}}\right)^{-\mathrm{T}}=\left(\mathbf{U}_{1}^{\mathrm{g}}\right)^{*} \mathbf{E}
$$


Em seguida, de acordo com (2), no caso sem ruído temos

$$
[\mathcal{Y}]_{(1)}^{\mathrm{T}}=\left[\left(\mathbf{C Q}_{\omega}\right) \diamond \mathbf{A}\right] \boldsymbol{\Gamma}
$$

e como $\hat{\Gamma}^{-\mathrm{T}}$ é dado em (18), definimos $\left(\mathbf{F}^{\mathrm{g}(2,3)}\right.$ como

$$
\begin{aligned}
\mathbf{F}^{\mathrm{g}(2,3)} & =[\mathcal{Y}]_{(1)}^{\mathrm{T}}\left(\mathbf{U}_{1}^{\mathrm{g}}\right)^{*} \mathbf{E}=\left[\left(\mathbf{C Q}_{\omega}\right)^{\mathrm{T}} \diamond \mathbf{A}\right] \boldsymbol{\Gamma} \hat{\boldsymbol{\Gamma}}^{+} \\
& \approx\left[\left(\mathbf{C Q}_{\omega}\right)^{\mathrm{T}} \diamond \mathbf{A}\right] \in \mathbb{C}^{Q M \times L} .
\end{aligned}
$$

\section{Técnica Proposta}

Para a realização da estimação de fatores para a realização da estimação de atraso propomos a utilização da Estrutura Semi-Algébrica para Decomposição Canonical Polyadic Aproximadas Através de Diagonalizações Simultâneas para Estimação de Atraso, do inglês Semi-algebraic framework for approximate Canonical Polyadic Decomposition via simultaneous matrix diagonalizations (SECSI) [18, 19, 20] em conjunto com a decomposição de valores singulares de alta ordem, do inglês Higher Order Singular Value Decomposition (HOSVD). Dessa forma o método SECSI aplica o HOSVD ao sinal recebido $\mathcal{Y}$

$$
\mathcal{Y}=\mathcal{S}^{\mathrm{c}} \times{ }_{1} \mathrm{U}_{1}^{\mathrm{c}} \times{ }_{2} \mathrm{U}_{2}^{\mathrm{c}} \times{ }_{3} \mathrm{U}_{3}^{\mathrm{c}}
$$

em que $\mathcal{S}^{\mathrm{c}} \in \mathbb{C}^{L_{d} \times L_{d} \times L_{d}}, \mathbf{U}_{1}^{\mathrm{c}} \in \mathbb{C}^{K \times L_{d}}, \mathbf{U}_{2}^{\mathrm{c}} \in \mathbb{C}^{Q \times L_{d}}$, e $\mathbf{U}_{3}^{\mathrm{c}} \in \mathbb{C}^{M \times L_{d}}$. Logo, $\mathcal{S}^{\mathrm{c}}$ pode ser representado como:

$$
\mathcal{S}^{\mathrm{c}}=\mathcal{I} \times{ }_{1} \mathbf{T}_{1}^{\mathrm{c}} \times_{2} \mathbf{T}_{2}^{\mathrm{c}} \times_{3} \mathbf{T}_{3}^{\mathrm{c}}
$$

onde $\mathbf{T}_{1}^{\mathrm{c}} \in \mathbb{C}^{L_{d} \times L_{d}}, \mathbf{T}_{2}^{\mathrm{c}} \in \mathbb{C}^{L_{d} \times L_{d}}, \mathrm{e} \mathbf{T}_{3}^{\mathrm{c}} \in \mathbb{C}^{L_{d} \times L_{d}}$. Logo

$$
\begin{array}{r}
\mathbf{U}_{1}^{\mathrm{c}} \mathbf{T}_{1}^{\mathrm{c}}=\mathbf{\Gamma}^{\mathrm{T}}, \\
\mathbf{U}_{2}^{\mathrm{c}} \mathbf{T}_{2}^{\mathrm{c}}=\left(\mathbf{C Q}_{\omega}\right)^{\mathrm{T}}, \\
\mathbf{U}_{3}^{\mathrm{c}} \mathbf{T}_{3}^{\mathrm{c}}=\mathbf{A}^{\mathrm{T}} .
\end{array}
$$

Uma vez que ao realizar a diagonalização simultânea do tensor $\mathcal{S}^{\mathrm{c}}$, criamos vários problemas de diagonalização à serem resolvidos, um tensor de terceira ordem produz seis fatores a serem estimados, ou seja, temos duas estimativas para cada dimensão do tensor $\mathcal{S}^{\mathrm{c}}$. Sendo assim, para realizar a diagonalização simultânea primeiramente deve-se calcular as fatias de primeiro-, segundo-, e terceiro-modo da $i$-ésima fatia do $\mathcal{S}^{\mathrm{c}}$. Assim, temos a fatia do 
terceiro-modo do tensor $\mathcal{S}^{\mathrm{c}}$ representada como:

$$
\begin{aligned}
\mathbf{S}_{3, i}^{c} & =\left[\left(\mathcal{S}^{\mathrm{c}} \times{ }_{3} \mathbf{U}_{3}^{\mathrm{c}}\right) \times{ }_{3} \mathbf{e}_{i}^{\mathrm{T}}\right] \\
& =\mathbf{T}_{1}^{\mathrm{c}} \operatorname{diag}\left\{\mathbf{A}^{\mathrm{H}}(:, i)\right\}\left(\mathbf{T}_{2}^{\mathrm{c}}\right)^{\mathrm{T}},
\end{aligned}
$$

a fatia do segundo-modo de $\mathcal{S}^{\mathrm{c}}$

$$
\begin{aligned}
\mathbf{S}_{2, i} & =\left[\left(\mathcal{S}^{\mathrm{c}} \times_{2} \mathbf{U}_{2}^{\mathrm{c}}\right) \times_{n} \mathbf{e}_{i}^{\mathrm{T}}\right] \\
& =\mathbf{T}_{1}^{\mathrm{c}} \operatorname{diag}\left\{\left(\mathbf{C} \mathbf{Q}_{\omega}\right)^{\mathrm{H}}(:, i)\right\}\left(\mathbf{T}_{3}^{\mathrm{c}}\right)^{\mathrm{T}},
\end{aligned}
$$

e, finalmente, temos a fatia do primeiro-modo

$$
\begin{aligned}
\mathbf{S}_{1, i}^{c} & =\left[\left(\mathcal{S}^{\mathrm{c}} \times_{1} \mathbf{U}_{1}^{\mathrm{c}}\right) \times_{1} \mathbf{e}_{i}^{\mathrm{T}}\right] \\
& =\mathbf{T}_{2}^{\mathrm{c}} \operatorname{diag}\left\{\boldsymbol{\Gamma}^{\mathrm{H}}(:, i)\right\}\left(\mathbf{T}_{3}^{\mathrm{c}}\right)^{\mathrm{T}},
\end{aligned}
$$

$\log 0$

$$
\begin{aligned}
& \mathbf{S}_{3, p}^{c}=\mathbf{T}_{1}^{\mathrm{c}} \operatorname{diag}\left\{\mathbf{A}^{\mathrm{H}}(:, p)\right\}\left(\mathbf{T}_{2}^{\mathrm{c}}\right)^{\mathrm{T}}, \\
& \mathbf{S}_{2, p}^{c}=\mathbf{T}_{1}^{\mathrm{c}} \operatorname{diag}\left\{\left(\mathbf{C Q}_{\omega}\right)^{\mathrm{H}}(:, p)\right\}\left(\mathbf{T}_{3}^{\mathrm{c}}\right)^{\mathrm{T}}, \\
& \mathbf{S}_{1, p}^{c}=\mathbf{T}_{2}^{\mathrm{c}} \operatorname{diag}\left\{\boldsymbol{\Gamma}^{\mathrm{H}}(:, p)\right\}\left(\mathbf{T}_{3}^{\mathrm{c}}\right)^{\mathrm{T}},
\end{aligned}
$$

em que $\mathbf{e}_{i}^{\mathrm{T}}$ é um vetor de zeros exceto na $i$-ésima posição, e $p$ é um índice arbitrário entre um e o total de fatias à serem diagonalizadas:

$$
p=\arg \min _{i} \operatorname{cond}\left\{\mathbf{S}_{n, i}^{\mathrm{c}}\right\}
$$

em que cond $\{\cdot\}$ calcula o número condicional da matriz. Quanto menor o número condicional, maior a estabilidade da inversão da matriz. Dessa forma, selecionamos a matriz com o menor número condicional.

Portanto, com as fatias de cada modo definidas, podemos calcular as matrizes à direita e à esquerda correspondentes às fatias de cada modo. Observe que a diagonalização simultânea da fatia do terceiro-modo é similar ao caso GPD-GEVD do estado-da-arte. Uma vez que $p$ é fixado, podemos variar todos os valores de $i$, assim obtendo $N-1$ equações, já que $i \neq p$.

Assim sendo, primeiramente, define-se a matriz à direita para a fatia do terceiro-modo da 
seguinte forma:

$$
\begin{aligned}
\mathbf{S}_{3, i}^{\mathrm{c}, \text { rhs }} & =\mathbf{S}_{3, i}^{\mathrm{c}}\left(\mathbf{S}_{3, p}^{\mathrm{c}}\right)^{-1} \\
& =\mathbf{T}_{1}^{\mathrm{c}} \operatorname{diag}\left\{\mathbf{A}^{\mathrm{H}}(:, i) \mathbf{A}^{\mathrm{H}}(:, p)\right\}\left(\mathbf{T}_{1}^{\mathrm{c}}\right)^{-1} \\
& =\mathbf{T}_{1}^{\mathrm{c}} \mathbf{A}^{\mathrm{H}}\left(\mathbf{T}_{1}^{\mathrm{c}}\right)^{-1}
\end{aligned}
$$

em seguida, defini-se a matriz à esquerda para a fatia do terceiro-modo como:

$$
\begin{aligned}
\mathbf{S}_{3, i}^{\mathrm{c}, \text { lhs }} & =\left(\left(\mathbf{S}_{3, p}^{\mathrm{c}}\right)^{-1} \mathbf{S}_{3, i}^{\mathrm{c}}\right)^{\mathrm{T}}=\left(\mathbf{S}_{3, i}^{\mathrm{c}}\right)^{\mathrm{T}}\left(\mathbf{S}_{3, p}^{\mathrm{c}}\right)^{-T} \\
& =\mathbf{T}_{1}^{\mathrm{c}} \operatorname{diag}\left\{\mathbf{A}^{\mathrm{H}}(:, i) \mathbf{A}^{\mathrm{H}}(:, p)\right\}\left(\mathbf{T}_{2}^{\mathrm{c}}\right)^{-1} \\
& =\mathbf{T}_{2}^{\mathrm{c}} \mathbf{A}^{\mathrm{H}}\left(\mathbf{T}_{2}^{\mathrm{c}}\right)^{-1} .
\end{aligned}
$$

Dessa forma, transformamos a fatia de terceiro modo em dois problemas de diagonalização simultânea. Portanto, é possível obter uma estimativa de $\mathbf{A}$ da diagonalização simultânea de $\mathbf{S}_{3, i}^{\text {c,rrs }}$. Adicionalmente, obtêm-se uma estimativa de $\mathbf{A}$ à partir da diagonalização simultânea de $\mathbf{S}_{3, i}^{\text {cllhs }}$. Além disso, as matrizes que diagonalizam $\mathbf{S}_{3, i}^{\text {c,rhs }}$ e $\mathbf{S}_{3, i}^{\mathrm{c}, \text { lhs }}$ resultam em estimativas para $\mathbf{T}_{1}^{\mathrm{c}}$, e $\mathbf{T}_{2}^{\mathrm{c}}$. Assim, podemos obter estimativas para $\mathbf{U}_{1}^{\mathrm{c}} \mathbf{T}_{1}^{\mathrm{c}}=\hat{\boldsymbol{\Gamma}}^{\mathrm{T}}, \mathrm{e} \mathbf{U}_{2}^{\mathrm{c}} \mathbf{T}_{2}^{\mathrm{c}}=\left(\hat{\mathbf{C}} \mathbf{Q}_{\omega}\right)^{\mathrm{T}}$.

De forma similar, para a fatia do segundo-modo, calcula-se a matriz à direita da seguinte forma:

$$
\begin{aligned}
\mathbf{S}_{2, i}^{\mathrm{c}, \mathrm{rhs}} & =\mathbf{S}_{2, i}^{\mathrm{c}}\left(\mathbf{S}_{2, p}^{\mathrm{c}}\right)^{-1} \\
& =\mathbf{T}_{1}^{\mathrm{c}} \operatorname{diag}\left\{\left(\mathbf{C Q}_{\omega}\right)^{\mathrm{H}}(:, i)\left(\mathbf{C Q}_{\omega}\right)^{\mathrm{H}}(:, p)\right\}\left(\mathbf{T}_{1}^{\mathrm{c}}\right)^{-1} \\
& =\mathbf{T}_{1}^{\mathrm{c}}\left(\mathbf{C} \mathbf{Q}_{\omega}\right)^{\mathrm{H}}\left(\mathbf{T}_{1}^{\mathrm{c}}\right)^{-1}
\end{aligned}
$$

em seguida, define-se a matriz à esquerda da fatia do segundo modo como

$$
\begin{aligned}
\mathbf{S}_{2, i}^{\mathrm{c} l \mathrm{lhs}} & =\left(\left(\mathbf{S}_{2, p}^{\mathrm{c}}\right)^{-1} \mathbf{S}_{2, i}^{\mathrm{c}}\right)^{T}=\left(\mathbf{S}_{2, i}^{\mathrm{c}}\right)^{\mathrm{T}}\left(\mathbf{S}_{2, p}^{\mathrm{c}}\right)^{-T} \\
& =\mathbf{T}_{3}^{\mathrm{c}} \operatorname{diag}\left\{\left(\mathbf{C Q}_{\omega}\right)^{\mathrm{H}}(:, i)\left(\mathbf{C} \mathbf{Q}_{\omega}\right)^{\mathrm{H}}(:, p)\right\}\left(\mathbf{T}_{3}^{\mathrm{c}}\right)^{-1} \\
& =\mathbf{T}_{3}^{\mathrm{c}}\left(\mathbf{C} \mathbf{Q}_{\omega}\right)^{\mathrm{H}}\left(\mathbf{T}_{3}^{\mathrm{c}}\right)^{-1}
\end{aligned}
$$

Novamente, criamos dois problemas de diagonalização simultânea para a fatia do segundo-modo. Portanto, podemos adquirir duas estimativas de $\left(\mathrm{CQ}_{\omega}\right)$ à partir da diagonalização simultânea de $\mathbf{S}_{2, i}^{\text {c,rhs }}$ e $\mathbf{S}_{2, i}^{\text {clhs }}$. Além disso, a diagonalização simultânea produz as estimativas para $\mathbf{T}_{1}^{\mathrm{c}}$ e $\mathbf{T}_{3}^{\mathrm{c}}$. Com isso, podemos estimar as matrizes de fatores $\mathbf{U}_{1}^{\mathrm{c}} \mathbf{T}_{1}^{\mathrm{c}}=\hat{\boldsymbol{\Gamma}}^{\mathrm{T}}$, e $\mathbf{U}_{3}^{c} \mathbf{T}_{3}^{\mathrm{c}}=\hat{\mathbf{A}}^{\mathrm{T}}$.

Finalmente, define-se a matriz à direita e a matriz à esquerda correspondentes à fatia do 
primeiro-modo. Novamente, primeiro define-se a matriz à direita da seguinte forma

$$
\begin{aligned}
\mathbf{S}_{1, i}^{\mathrm{c}, \mathrm{rhs}} & =\mathbf{S}_{1, i}^{\mathrm{c}}\left(\mathbf{S}_{1, p}^{\mathrm{c}}\right)^{-1} \\
& =\mathbf{T}_{2}^{\mathrm{c}} \operatorname{diag}\left\{\boldsymbol{\Gamma}^{\mathrm{H}}(:, i) \boldsymbol{\Gamma}^{\mathrm{H}}(:, p)\right\}\left(\mathbf{T}_{2}^{\mathrm{c}}\right)^{-1} \\
& =\mathbf{T}_{2}^{\mathrm{c}} \boldsymbol{\Gamma}^{\mathrm{H}}\left(\mathbf{T}_{2}^{\mathrm{c}}\right)^{-1},
\end{aligned}
$$

em seguida define-se a matriz à esquerda

$$
\begin{aligned}
\mathbf{S}_{1, i}^{\mathrm{c}, \text { hhs }} & =\left(\left(\mathbf{S}_{1, p}^{\mathbf{c}}\right)^{-1} \mathbf{S}_{1, i}^{\mathrm{c}}\right)^{\mathrm{T}}=\left(\mathbf{S}_{1, i}^{\mathrm{c}}\right)^{\mathrm{T}}\left(\mathbf{S}_{1, p}^{\mathrm{c}}\right)^{-T} \\
& =\mathbf{T}_{3}^{\mathrm{c}} \operatorname{diag}\left\{\boldsymbol{\Gamma}^{\mathrm{H}}(:, i) \boldsymbol{\Gamma}^{\mathrm{H}}(:, p)\right\}\left(\mathbf{T}_{3}^{\mathrm{c}}\right)^{-1} \\
& =\mathbf{T}_{3}^{\mathrm{c}} \boldsymbol{\Gamma}^{\mathrm{H}}\left(\mathbf{T}_{3}^{\mathrm{c}}\right)^{-1}
\end{aligned}
$$

Portanto, temos dois problemas de diagonalização simultânea $\mathbf{S}_{1, i}^{\mathrm{c}, \text { rhs }}$ e $\mathbf{S}_{1, i}^{\mathrm{c}, \text { lhs }}$ que para a primeira fatia do primeiro modo resulta em duas estimativas para $\Gamma$. Adicionalmente, a diagonalização simultânea produz estimativas para $\mathbf{T}_{2}^{\mathrm{c}}$ e $\mathbf{T}_{3}^{\mathrm{c}}$. Assim, pode-se estimar as matrizes de fatores $\mathbf{U}_{2}^{\mathrm{c}} \mathbf{T}_{2}^{\mathrm{c}}=\left(\hat{\mathbf{C}} \mathbf{Q}_{\omega}\right)^{\mathrm{T}}$, e $\mathbf{U}_{3}^{\mathrm{c}} \mathbf{T}_{3}^{\mathrm{c}}=\hat{\mathbf{A}}^{\mathrm{T}}$.

Através da realização de diversas simulações utilizando todas a fatias de todos os modos descritos, descobriu-se que quando se tem um LOS e dois NLOS sinais iluminando o arranjo de antenas a matriz à direita da fatia do terceiro-modo é a que produz o melhor resultado. Portanto, propõe-se utilizar somente a estimativa adquirida pela diagonalização simultânea de $\mathbf{S}_{3, i}^{\text {crrhs }}$. Após obter $\mathbf{S}_{3, i}^{\text {c,rhs }}$ podemos encontrar a matriz $\hat{\mathbf{T}}_{1}^{\mathrm{c}}$ que diagonaliza simultaneamente as $N-1$ equações utilizando [21] e [22]. Então, utilizamos $\mathbf{U}_{1}^{\mathrm{c}}$ de (21) para estimar $\hat{\boldsymbol{\Gamma}}^{\mathrm{T}}$

$$
\mathbf{U}_{1}^{\mathrm{c}} \hat{\mathbf{T}}_{1}^{\mathrm{c}}=\hat{\boldsymbol{\Gamma}}^{\mathrm{T}}
$$

Após isso, de acordo com (2), no caso sem ruído temos

$$
[\mathcal{Y}]_{(1)}^{\mathrm{T}}=\left[\left(\mathbf{C Q}_{\omega}\right) \diamond \mathbf{A}\right] \boldsymbol{\Gamma}
$$

e uma vez que $\hat{\Gamma}^{\mathrm{T}}$ é dado em (39), definimos $\mathbf{F}^{\mathrm{c}(2,3)}$ como

$$
\begin{aligned}
\mathbf{F}^{\mathrm{c}(2,3)} & =[\mathcal{Y}]_{(1)}^{\mathrm{T}} \hat{\boldsymbol{\Gamma}}^{-\mathrm{T}}=\left[\left(\mathbf{C Q}_{\omega}\right)^{\mathrm{T}} \diamond \mathbf{A}\right] \boldsymbol{\Gamma} \hat{\boldsymbol{\Gamma}}^{+\mathrm{T}} \\
& \approx\left[\left(\mathbf{C} \mathbf{Q}_{\omega}\right)^{\mathrm{T}} \diamond \mathbf{A}\right] \in \mathbb{C}^{Q M \times L_{d}}
\end{aligned}
$$

As matrizes de fatores $\left(\hat{\mathbf{C}} \mathbf{Q}_{\omega}\right)^{\mathrm{T}}$ e $\hat{\mathbf{A}}$ podem ser estimadas à partir de (41) utilizando a Fatorização Khatri-Rao de mínimos quadrados, do inglês Least Squares Khatri-Rao Facto- 


\section{Estimação de Atraso}

Uma vez que as matrizes de fatores $\left(\hat{\mathbf{C}} \mathbf{Q}_{\omega}\right)^{\mathrm{T}}, \hat{\mathbf{A}}$, e $\hat{\boldsymbol{\Gamma}}$ foram estimadas estas são normalizadas para seus $\ell_{d}$-ésimo componente:

$$
\begin{aligned}
\left(\hat{\overline{\mathbf{C}}} \mathbf{Q}_{\omega}\right)_{\cdot, \ell_{d}}{ }^{\mathrm{T}} & =\left(\hat{\mathbf{C}} \mathbf{Q}_{\omega}\right)_{\cdot, \ell_{d}}{ }^{\mathrm{T}} /\left\|\left(\hat{\mathbf{C}} \mathbf{Q}_{\omega}\right)_{\cdot, \ell_{d}}{ }^{\mathrm{T}}\right\|_{F} \\
\hat{\overline{\mathbf{A}}}_{\cdot, \ell_{d}} & =\hat{\mathbf{A}}_{\cdot, \ell_{d}} /\left\|\hat{\mathbf{A}}_{\cdot, \ell_{d}}\right\|_{F} \\
\hat{\overline{\boldsymbol{\Gamma}}} & =\hat{\boldsymbol{\Gamma}}_{\cdot, \ell_{d}} /\left\|\hat{\boldsymbol{\Gamma}}_{\cdot, \ell_{d}}\right\|_{F} .
\end{aligned}
$$

Em seguida, constrói-se um tensor $\mathcal{G}_{\ell}$ correspondente à partir $\ell_{d}$-ésimo componente das matrizes de fatores normalizadas em (44)

$$
\mathcal{G}_{\ell_{d}}=\hat{\overline{\boldsymbol{\Gamma}}}_{\cdot, \ell_{d}} \circ\left(\hat{\overline{\mathbf{C}}}_{\mathbf{Q}_{\omega}}\right)_{\cdot, \ell_{d}}^{\mathrm{T}} \circ \hat{\overline{\mathbf{A}}}_{\cdot, \ell_{d}}
$$

onde $\mathcal{G}_{\ell_{d}} \in \mathbb{C}^{K \times Q \times M}$. Em seguida, armazena-se o tensor $\mathcal{G}_{\ell_{d}}$ correspondente ao $\ell_{d}$-ésimo componente em uma matriz

$$
\mathbf{G}_{\cdot, \ell_{d}}=\operatorname{vec}\left\{\mathcal{G}_{\ell_{d}}\right\}
$$

onde $\mathbf{G} \in \mathbb{C}^{K Q M \times L_{d}}$. Assim, pode-se calcular as amplitudes através da multiplicação da pseudo-inversa de $\mathrm{G}$ pelo tensor $\mathcal{Y}$ :

$$
\gamma=\mathrm{G}^{+} \operatorname{vec}\{\mathcal{Y}\}
$$

Em seguida, para encontrar a coluna de $\left(\hat{\overline{\mathbf{C}}} \mathbf{Q}_{\omega}\right)$ que corresponde ao componente de linha de visada, aplica-se arg max à $\gamma$ e escolhe-se a $s_{\text {Los }}$ coluna com a maior amplitude.

$$
s_{\mathrm{LOS}}=\arg \max \gamma
$$

Depois, usa-se $s_{\text {LOS }}$ para selecionar o componente LOS da matriz estimada $\left(\hat{\overline{\mathbf{C}}} \mathbf{Q}_{\omega}\right)^{\mathrm{T}}$ e a multiplica por $\Sigma \mathbf{V}^{\mathrm{H}}$ obtida através da aplicação da decomposição em valores singulares econômica à $\mathrm{Q}$

$$
\mathbf{q}=\left[\left(\hat{\overline{\mathbf{C}}} \mathbf{Q}_{\omega}\right)_{\cdot, s_{\mathrm{LOS}}}{ }^{\mathrm{T}} \boldsymbol{\Sigma} \mathbf{V}^{\mathrm{H}}\right]^{\mathrm{T}}
$$


Finalmente, o vetor q é interpolado utilizando a interpolação de spline cúbica para alcançar maior precisão na estimação de atraso.

\section{Simulação}

Para a realização da simulação é utilizado um arranjo linear uniforme, do inglês Uniform Linear Array (ULA), centro-hermitiano com $M=8$ elementos espaçados por meio comprimento de onda. São considerados a recepção de dois sinais GNSS, o primeiro é um código pseudo-aleatório C/A do GPS enquanto o segundo é um código pseudo-aleatório da L1C do canal piloto do GPS, em que ambos possuem uma portadora de frequência $f_{c}=1575.42$ MHz. O código L1C do GPS possui uma duração total de transmissão $t_{3 \mathrm{rd}}=10 \mathrm{~ms}$, uma largura de banda $B_{3 \mathrm{rd}}=12.276 \mathrm{MHz}$, a maior largura de banda é consequência da modulação TMBOC. Com isso, para se ter uma comparação justa, [6] propôs o aumento da largura de banda do código C/A para $B_{2 \text { nd }}=B_{3 \text { rd }}$. Ambos os códigos são amostrado à cada $K$ ésimo período durante $K=30$ períodos com um período de duração de $\Delta \tau=10 \mathrm{~ms}$. Dessa forma, são coletadas $N=245520$ amostras para ambos códigos C/A e L1C.

Os componentes de LOS e NLOS são gerados com uma diferença de azimute de $\Delta \phi=60^{\circ}$. Adicionalmente, o componente LOS possui um atraso $\tau_{\mathrm{LOS}}$ enquanto o componente NLOS possui um atraso $\tau_{\mathrm{NLOS}}$ tal que $\tau_{\mathrm{NLOS}_{1}}=\tau_{\mathrm{LOS}}+\Delta \tau$ quando $L_{d}=2$ sinais são recebidos e quando temos $L_{d}=3$ sinais sendo recebidos se tem um segundo componente NLOS com um atraso $\tau_{\mathrm{NLOS}_{2}}=\tau_{\mathrm{NLOS}_{1}}+\Delta \tau$. Para ESPS a ULA é dividida em $L_{S}=5$ sub arranjos com $M_{S}=4$ elementos cada. A relação portadora-ruído é $C / N_{0}=48 \mathrm{~dB}-\mathrm{Hz}$, resultando numa razão sinal-ruído pós-correlação de $S N R_{\text {post }_{2 n d}} \approx 25 \mathrm{~dB}$ para GPS de segunda geração e uma razão sinal-ruído de $S N R_{\text {post }_{3 \mathrm{rd}}} \approx 25 \mathrm{~dB}$ para GPS de terceira geração.

Para realizar as simulações foi utilizado o esquema Monte Carlo com 2000 iterações. Dessa forma os resultados obtidos são expressados na forma do erro quadrático médio multiplicado pela velocidade da luz, em metros, para vários $\Delta \tau$. Os resultados são comparados com o caso ideal onde se tem o conhecimento do canal (A e $\tilde{\Gamma}$ ) e a correlação sem ruído do sinal LOS com o banco correlator [23].

\section{Estimação de Atraso Utilizando GPS de segunda geração}

Os resultados das simulações incluem a comparação da técnica CPD-GEVD do estadoda-arte com o método HOSVD SECSI para GPS de segunda geração. Alternativamente, para fins comparativos, durante as simulações foi adicionado a técnica HOOI SECSI que substitui o método de HOSVD. Assim, os resultados das simulações recebendo $L_{d}=2$ sinais e utilizando o primeiro fator estimado podem ser verificados na Figura 1. 


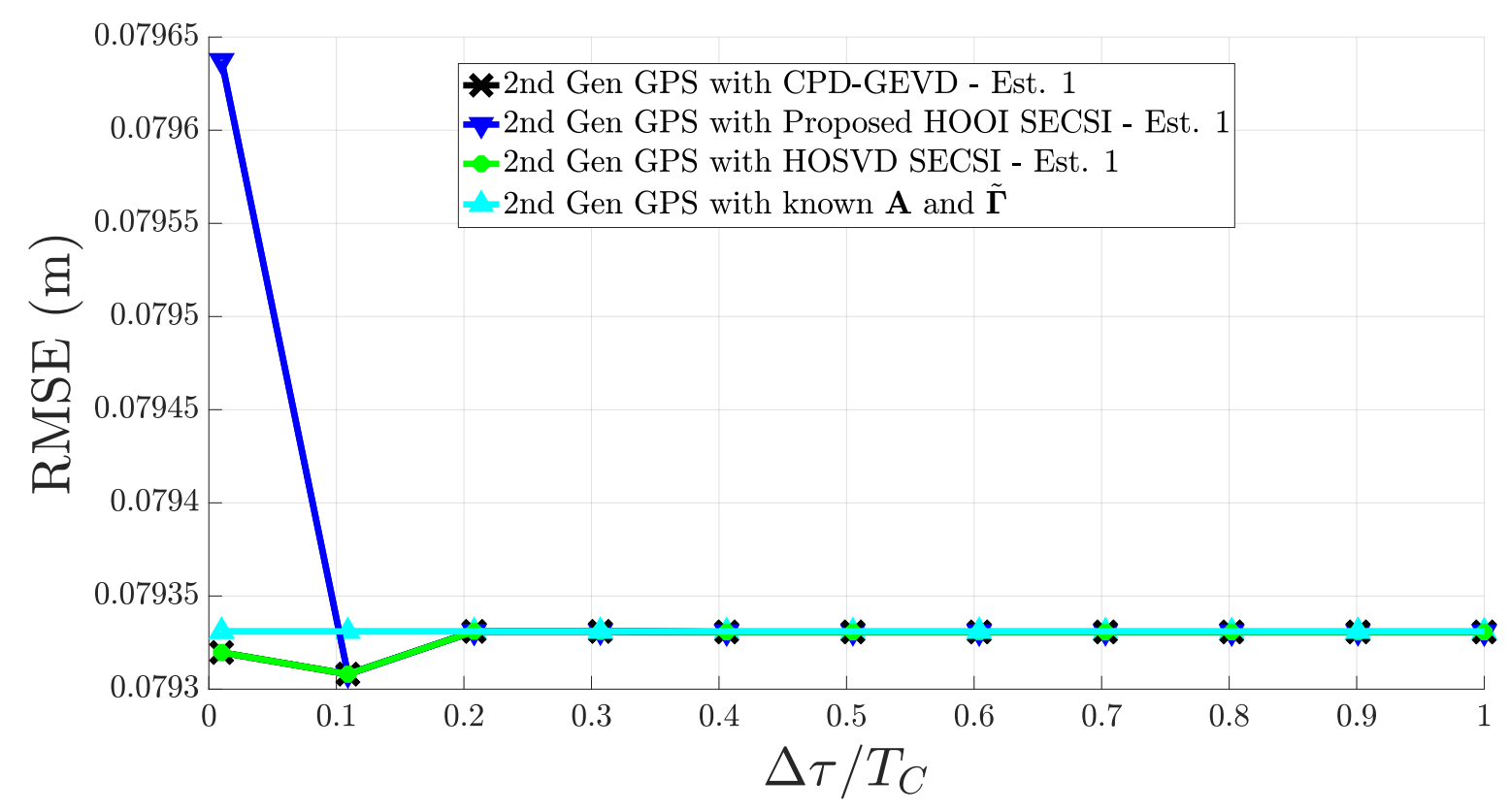

Figure 1: Simulação utilizando o primeiro fator estimado da técnica do estado-da-arte CPDGEVD, método HOSVD SECSI e método SECSI para GPS de segunda geração utilizando cada fator estimado com $M=8$ elementos e $L_{d}=2$ sinais recebidos. Em ambos os casos as amostras são coletadas por $K=30$ períodos que possuem $N=245520$ amostras.

Na Figura 2 é utilizado o segundo fator estimado pelas técnicas CPD-GEVD, HOSVD SECSI e HOOI SECSI.

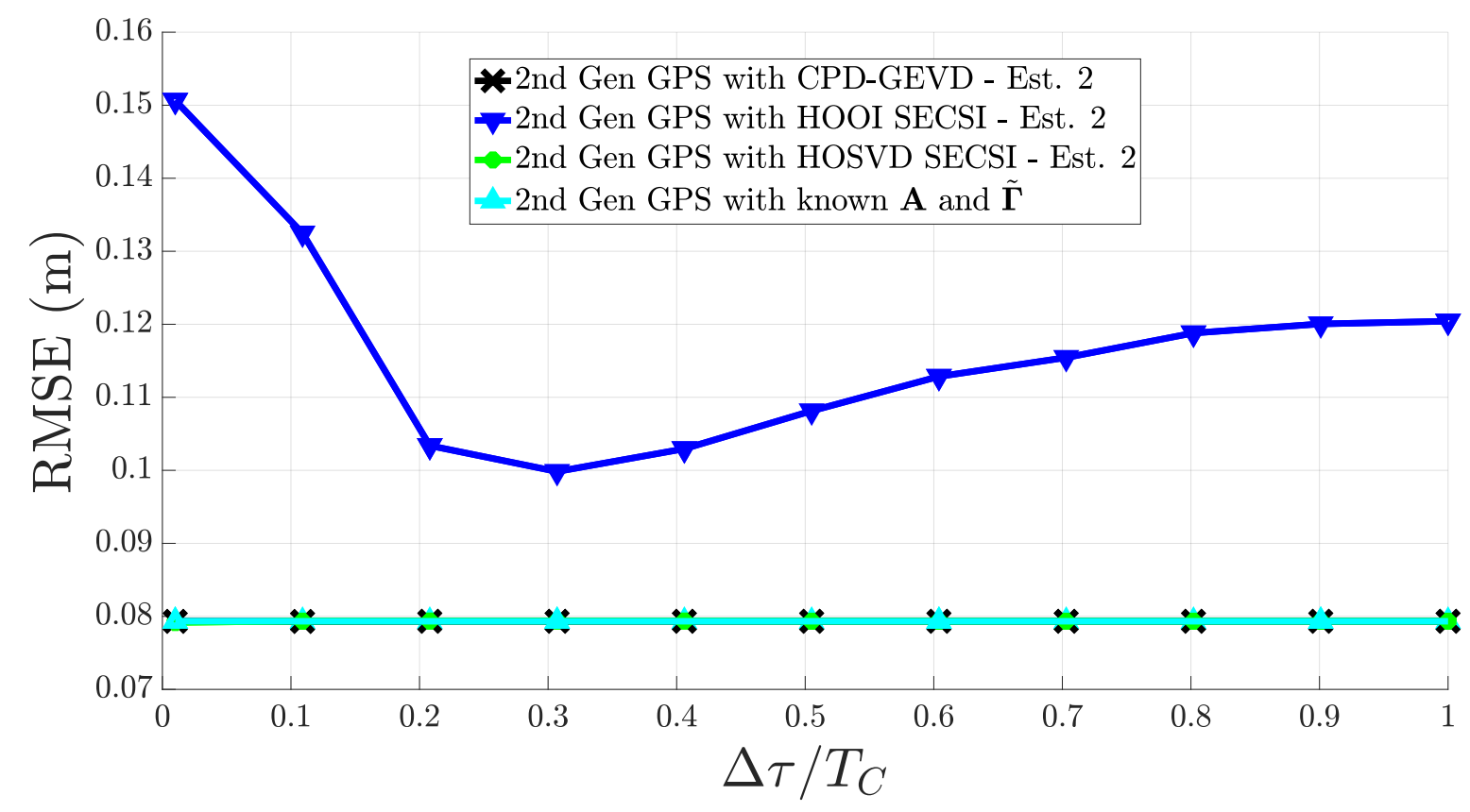

Figure 2: Simulação utilizando o segundo fator estimado da técnica do estado-da-arte CPDGEVD, método HOSVD SECSI e método SECSI para GPS de segunda geração utilizando cada fator estimado com $M=8$ elementos e $L_{d}=2$ sinais recebidos. Em ambos os casos as amostras são coletadas por $K=30$ períodos que possuem $N=245520$ amostras.

Figura 3 realiza a comparação do método CPD-GEVD do estado-da-arte com o método 
HOSVD SECSI e HOOI SECSI utilizando o terceiro fator estimado.

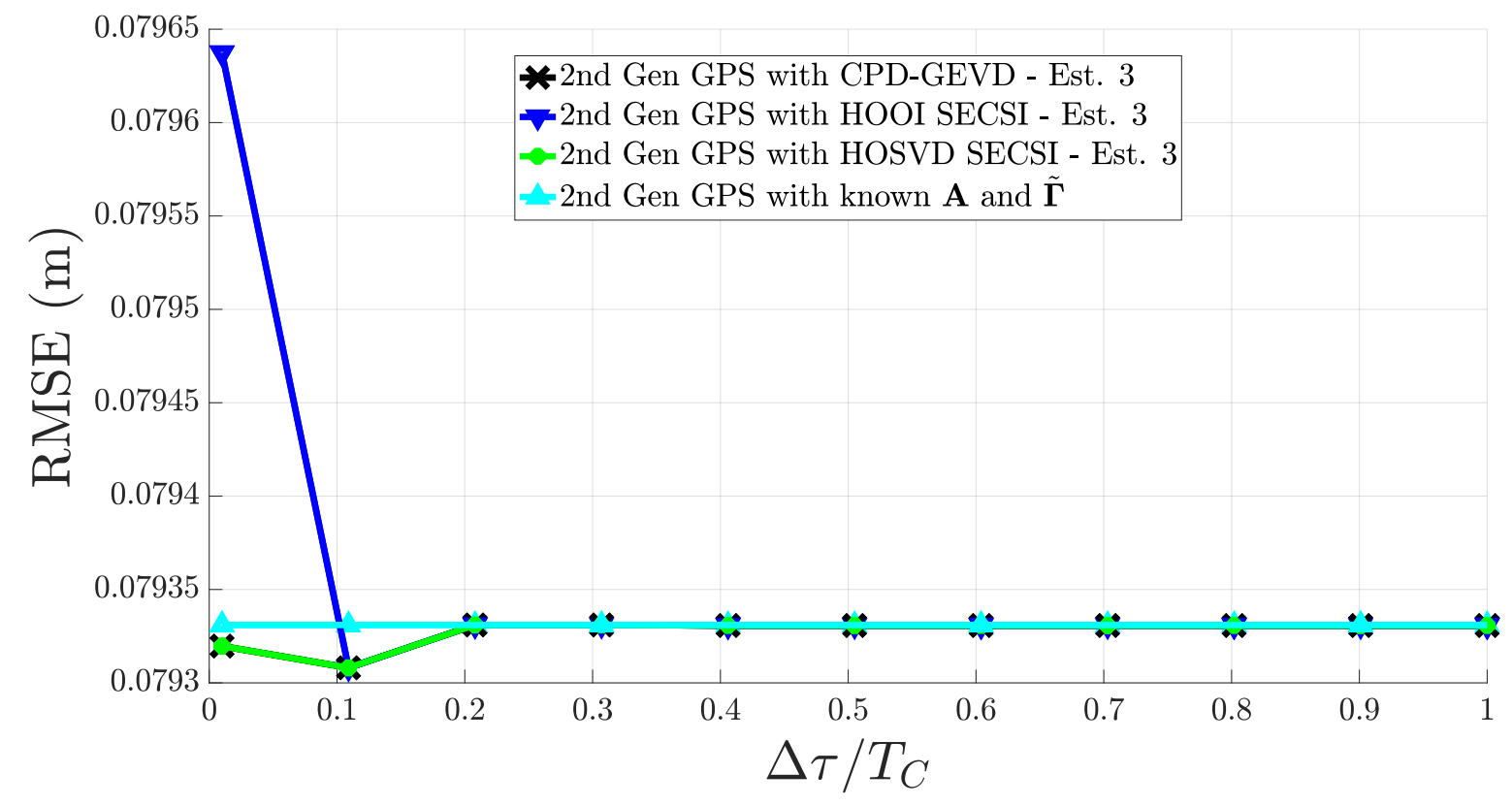

Figure 3: Simulação utilizando o terceiro fator estimado da técnica do estado-da-arte CPDGEVD, método HOSVD SECSI e método SECSI para GPS de segunda geração utilizando cada fator estimado com $M=8$ elementos e $L_{d}=2$ sinais recebidos. Em ambos os casos as amostras são coletadas por $K=30$ períodos que possuem $N=245520$ amostras.

Já na Figura 4 é realizada a comparação do método CPD-GEVD do estado-da-arte com o método HOSVD SECSI e HOOI SECSI utilizando o quarto fator estimado.

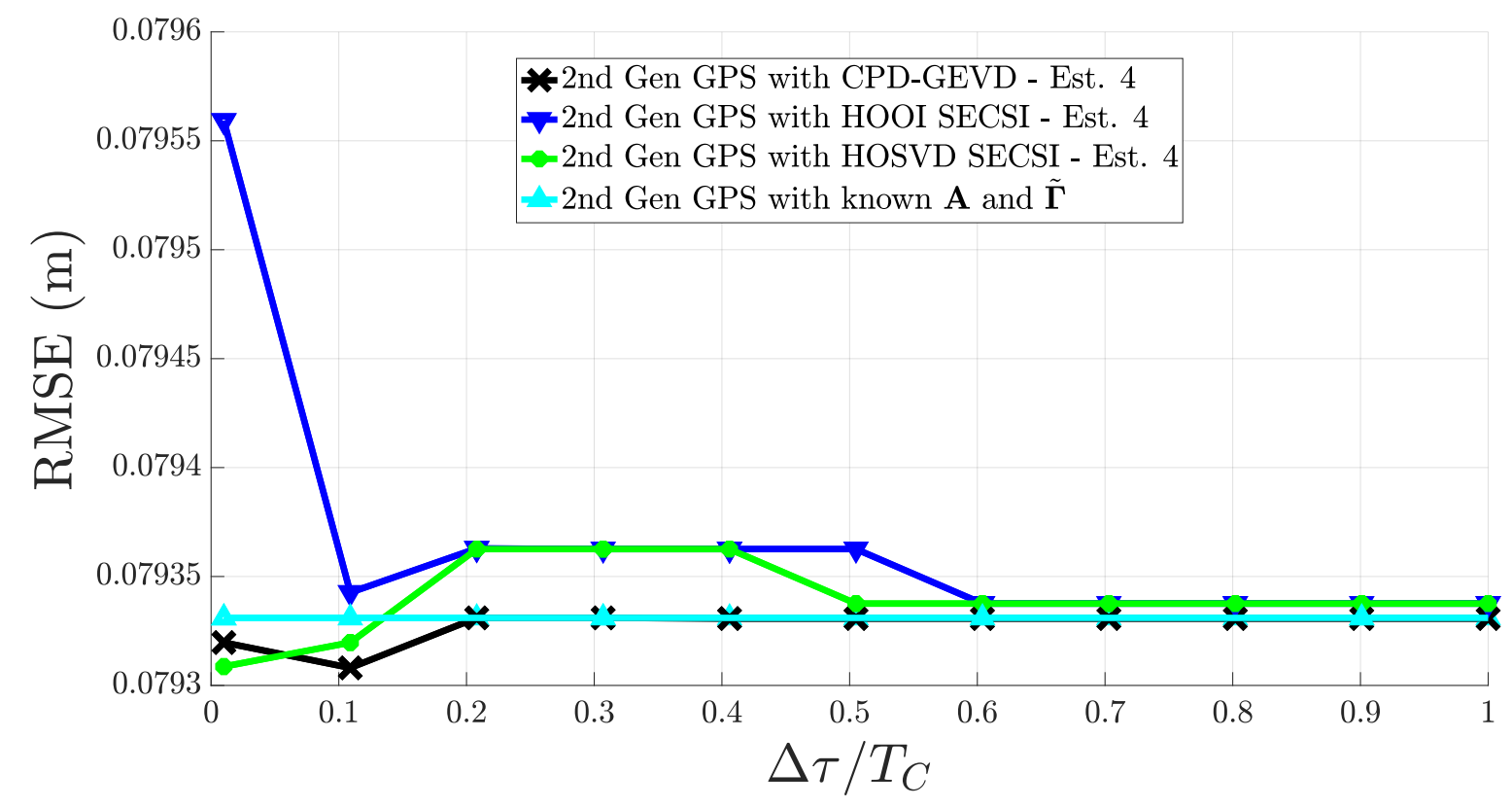

Figure 4: Simulação utilizando o quarto fator estimado da técnica do estado-da-arte CPDGEVD, método HOSVD SECSI e método SECSI para GPS de segunda geração utilizando cada fator estimado com $M=8$ elementos e $L_{d}=2$ sinais recebidos. Em ambos os casos as amostras são coletadas por $K=30$ períodos que possuem $N=245520$ amostras. 


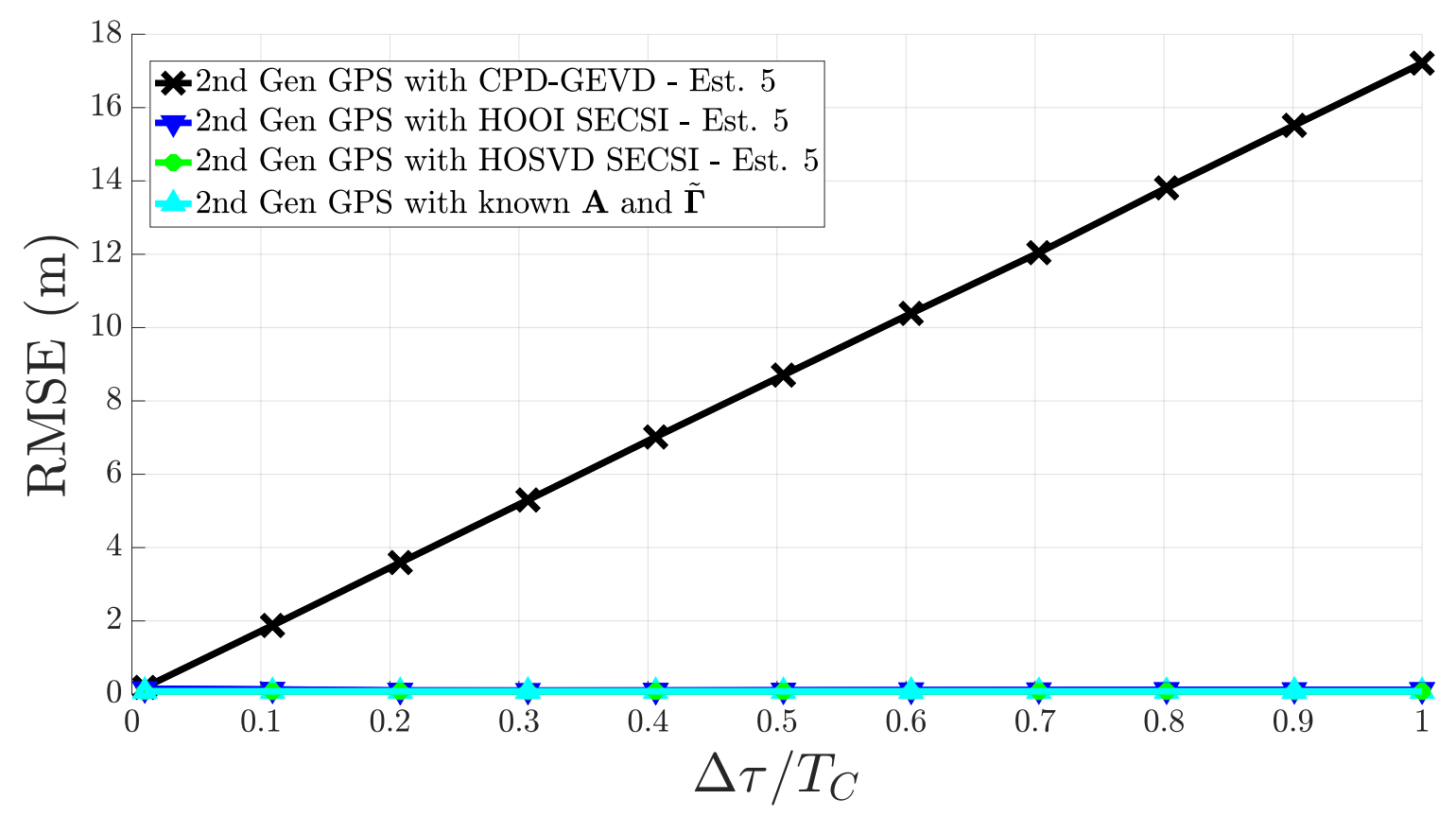

Figure 5: Simulação utilizando o quinto fator estimado da técnica do estado-da-arte CPDGEVD, método HOSVD SECSI e método SECSI para GPS de segunda geração utilizando cada fator estimado com $M=8$ elementos e $L_{d}=2$ sinais recebidos. Em ambos os casos as amostras são coletadas por $K=30$ períodos que possuem $N=245520$ amostras.

Figura 5 realiza a a comparação do método CPD-GEVD do estado-da-arte com o método HOSVD SECSI e HOOI SECSI utilizando o quinto fator estimado. Figura 6 exibe a comparação do método CPD-GEVD do estado-da-arte com o método HOSVD SECSI e HOOI SECSI utilizando o sexto fator estimado. 


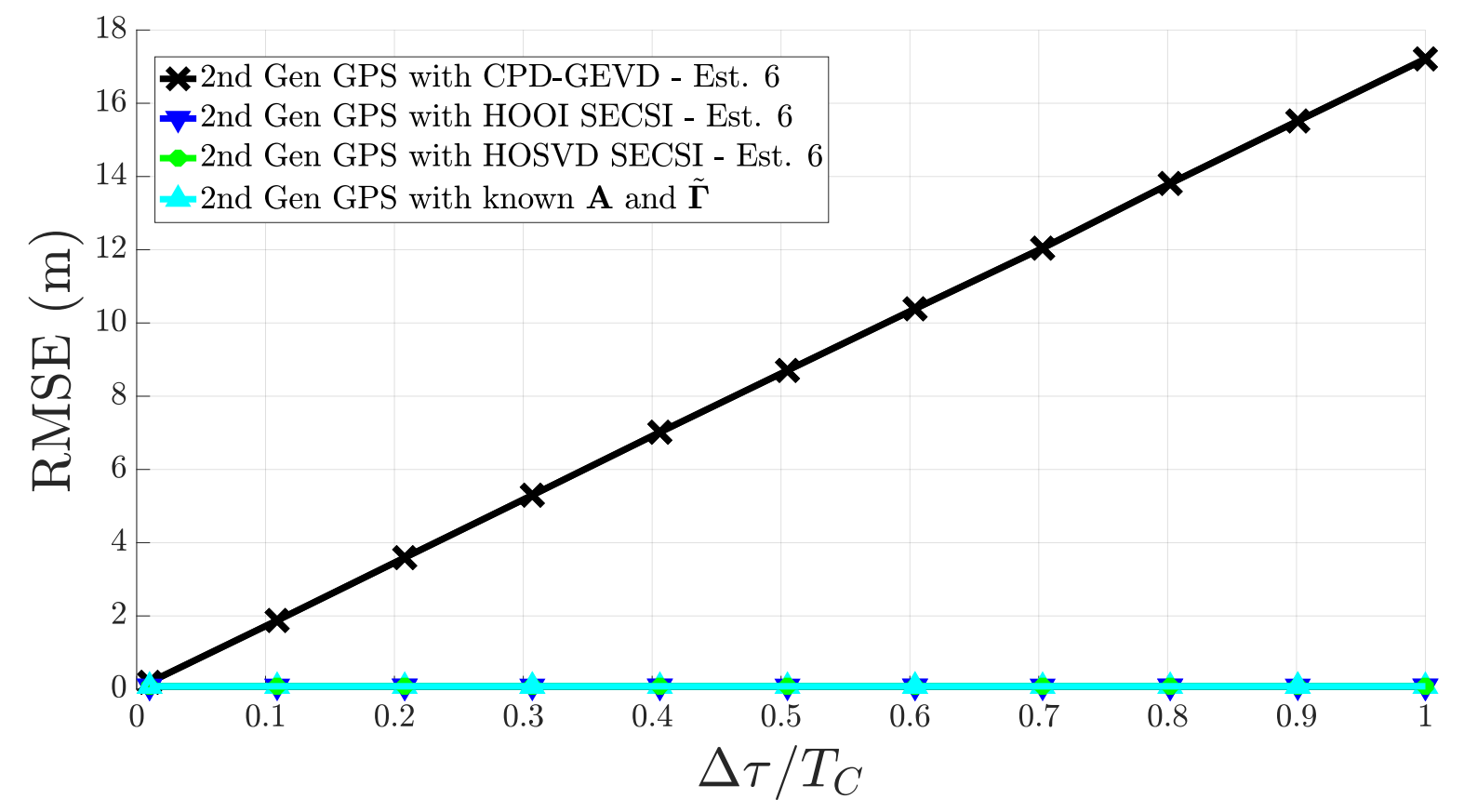

Figure 6: Simulação utilizando o sexto fator estimado da técnica do estado-da-arte CPDGEVD, método HOSVD SECSI e método SECSI para GPS de segunda geração utilizando cada fator estimado com $M=8$ elementos e $L_{d}=2$ sinais recebidos. Em ambos os casos as amostras são coletadas por $K=30$ períodos que possuem $N=245520$ amostras.

Como mostrado nas Figuras 1 à 6 o primeiro e terceiro estimador de fatores para CPDGEVD apresentam os melhores resultado, dessa forma decide-se manter o uso CPD-GEVD do estado da arte. Da mesma forma, é possível notar que o primeiro e terceiro estimador de fatores para SECSI apresentam os melhores resultados, assim o primeiro estimador de fatores foi escolhido para realizar as demais comparações. Na Figura 7 é realizada a comparação das técnicas do estado-da-arte com o método proposto para GPS de segunda geração quando recebendo $L_{d}=2$ sinais. Note que os métodos DoA/KRF, CPD-GEVD e o HOSVD SECSI consideravelmente superam a técnica HOSVD+FBA+ESPS. Onde HOSVD+FBA+ESPS apresenta um erro máximo de aproximadamente $0.28 \mathrm{~m}$ enquanto as demais técnicas apresentam um erro máximo de $0.079 \mathrm{~m}$. 


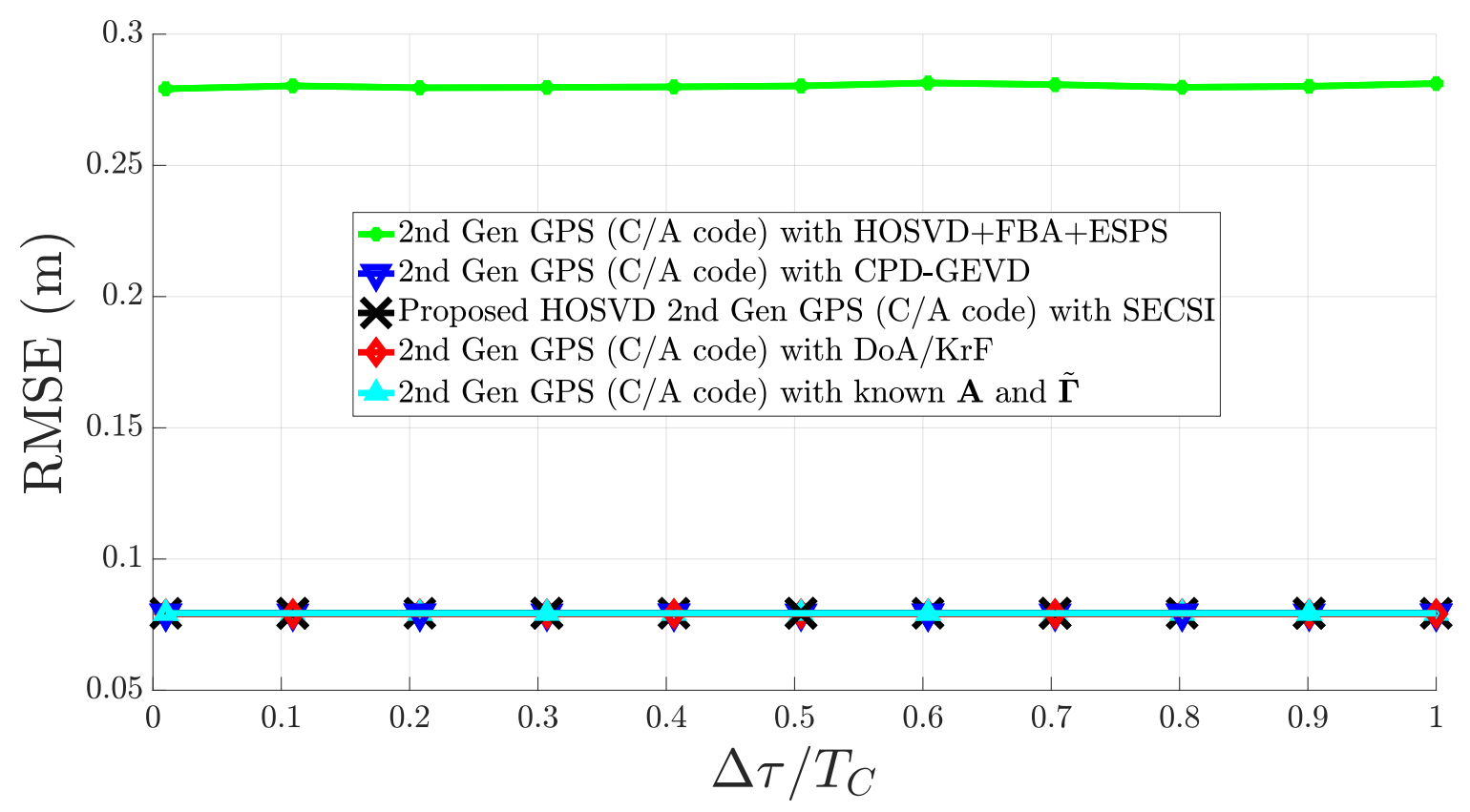

Figure 7: Simulação dos esquemas CPD-GEVD do estado-da-arte e do método SECSI proposto para GPS de segunda geração com $M=8$ elementos e $L_{d}=2$ sinais recebidos. Em ambos os casos as amostras são coletadas por $K=30$ períodos que possuem $N=245520$ amostras.

Já na Figura 8 é feita a comparação do método HOSVD+FBA+ESPS, DoA/KRF, e CPD-GEVD do estado-da-arte com o método SECSI proposto quando $L_{d}=3$. Observe que novamente as técnicas DoA/KRF, CPD-GEVD e o HOSVD SECSI superam o HOSVD+FBA+ESPS que apresenta um erro máximo de $0.28 \mathrm{~m}$. Ademais, note que o método CPD-GEVD apresenta pior performance quando $\Delta \tau<0.2 T_{c}$. Esse erro ocorre pela forte correlação entre as os sinais LOS e NLOS o que ocasiona um tensor de posto deficiente e pode ser solucionado ao utilizar técnicas de estimação de posto [24, 25, 26]. Também, note que a técnica SECSI apresenta um pico quando os sinais estão fortemente correlatados, porém SECSI mostra-se mais tolerante a deficiência do posto e consegue estimar o atraso com sucesso. 


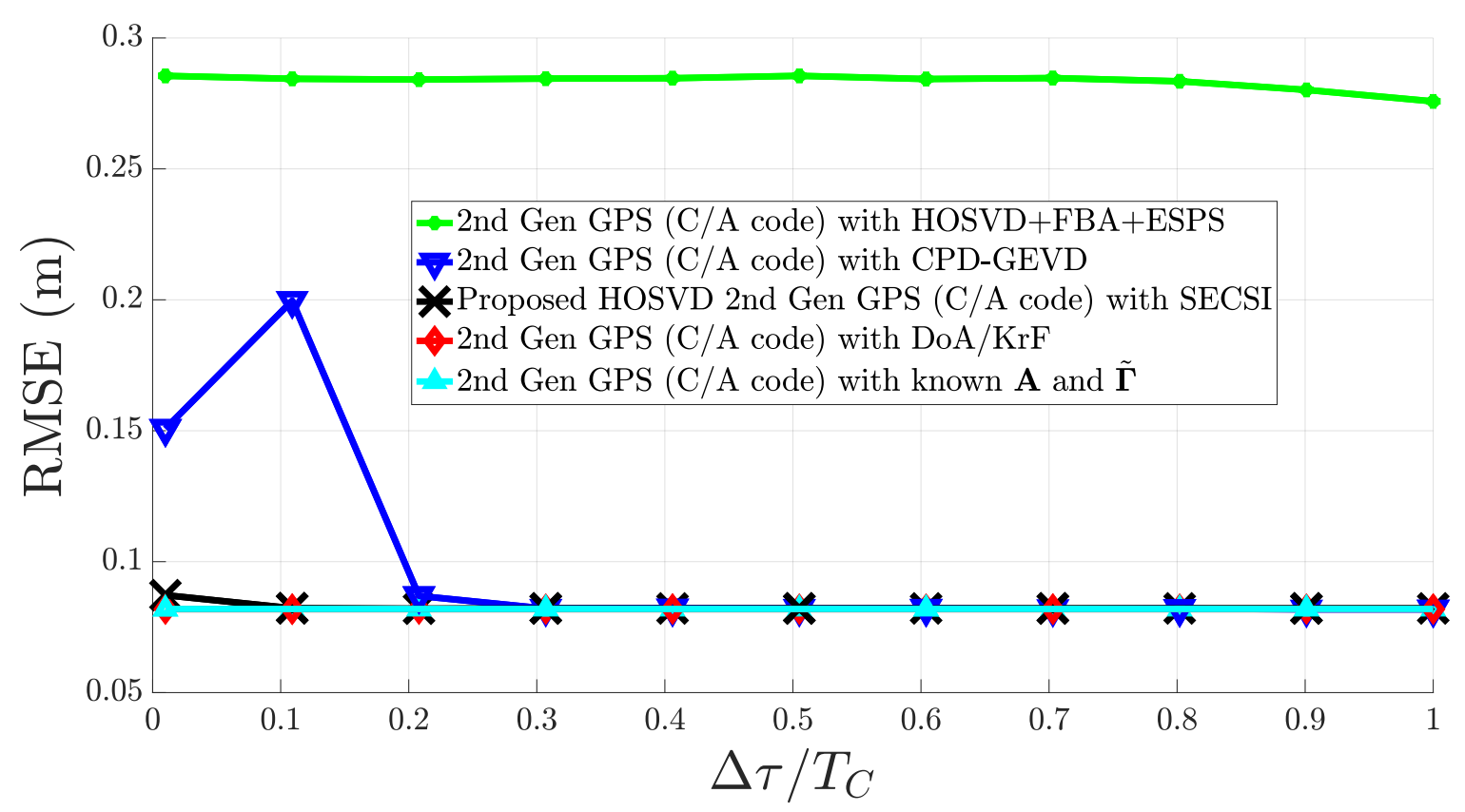

Figure 8: Simulação dos esquemas CPD-GEVD do estado-da-arte e do método SECSI proposto para GPS de segunda geração com $M=8$ elementos e $L_{d}=2$ sinais recebidos. Em ambos os casos as amostras são coletadas por $K=30$ períodos que possuem $N=245520$ amostras.

\section{Estimação de Atraso Utilizando GPS Terceira Geração}

Os resultados das simulações incluem: a comparação da técnica CPD-GEVD do estadoda-arte com o método HOSVD SECSI para o GPS de terceira geração. Novamente, para fins comparativos, durante as simulações foi adicionado a técnica HOOI SECSI que substitui o método HOSVD. Assim, os resultados das simulações recebendo $L_{d}=2$ sinais e utilizando o primeiro fator estimado na Figura 9. 


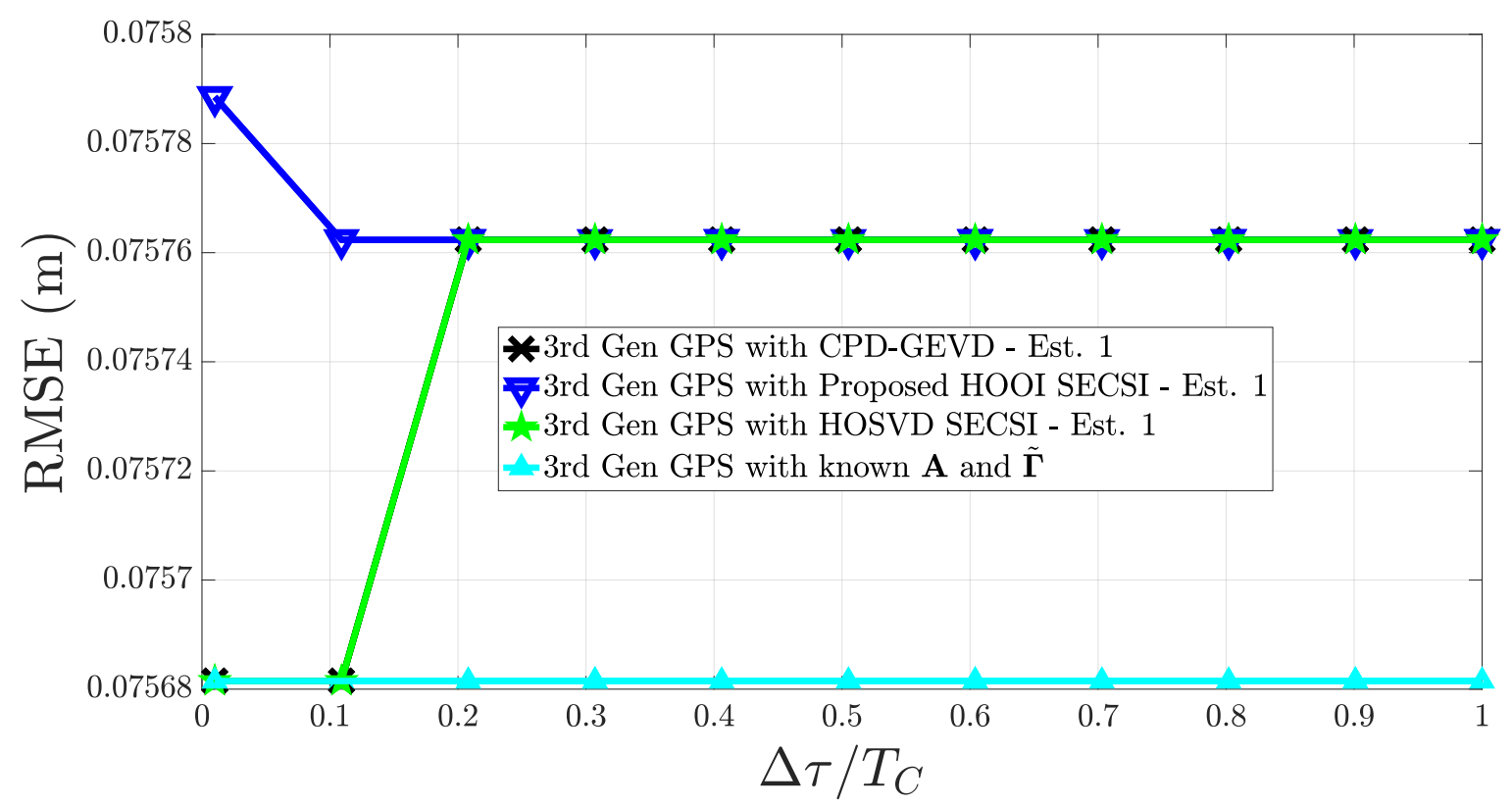

Figure 9: Simulação utilizando primeiro fator estimado da técnica do estado-da-arte CPDGEVD, método HOSVD SECSI e método SECSI para GPS de terceira geração utilizando cada fator estimado com $M=8$ elementos e $L_{d}=2$ sinais recebidos. Em ambos os casos as amostras são coletadas por $K=30$ períodos que possuem $N=245520$ amostras.

Na Figura 10 é utilizado o segundo fator estimado pelas técnicas CPD-GEVD, HOSVD SECSI e HOOI SECSI.

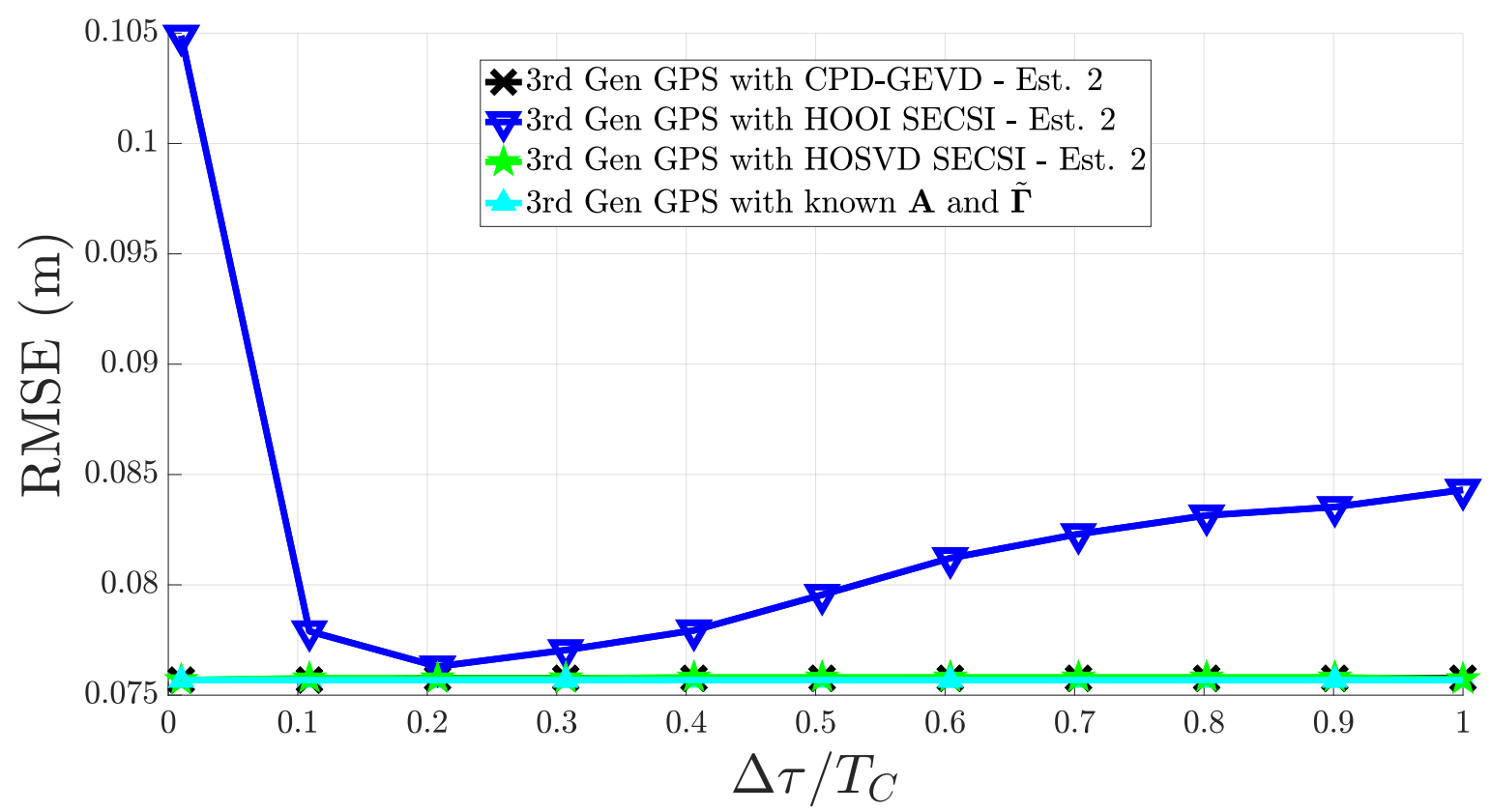

Figure 10: Simulação utilizando segundo fator estimado da técnica do estado-da-arte CPDGEVD, método HOSVD SECSI e método SECSI para GPS de terceira geração utilizando cada fator estimado com $M=8$ elementos e $L_{d}=2$ sinais recebidos. Em ambos os casos as amostras são coletadas por $K=30$ períodos que possuem $N=245520$ amostras.

Figura 11 exibe a comparação do método CPD-GEVD do estado-da-arte com o método 
HOSVD SECSI e HOOI SECSI utilizando o terceiro fator estimado.

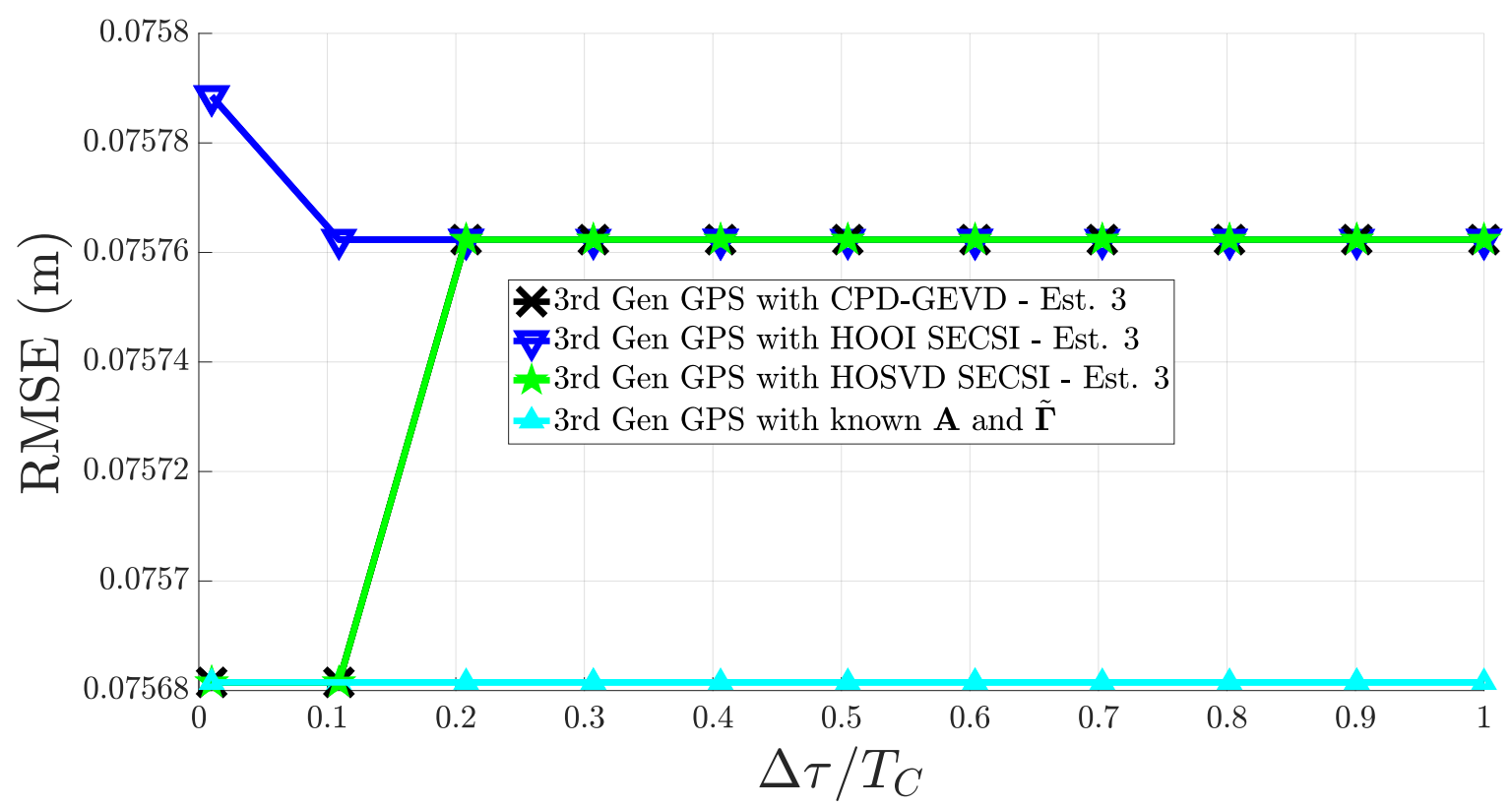

Figure 11: Simulação utilizando terceiro fator estimado da técnica do estado-da-arte CPDGEVD, método HOSVD SECSI e método SECSI para GPS de terceira geração utilizando cada fator estimado com $M=8$ elementos e $L_{d}=2$ sinais recebidos. Em ambos os casos as amostras são coletadas por $K=30$ períodos que possuem $N=245520$ amostras.

Já a comparação do método CPD-GEVD do estado-da-arte com o método HOSVD SECSI e HOOI SECSI utilizando o quarto fator estimado na Figura 12.

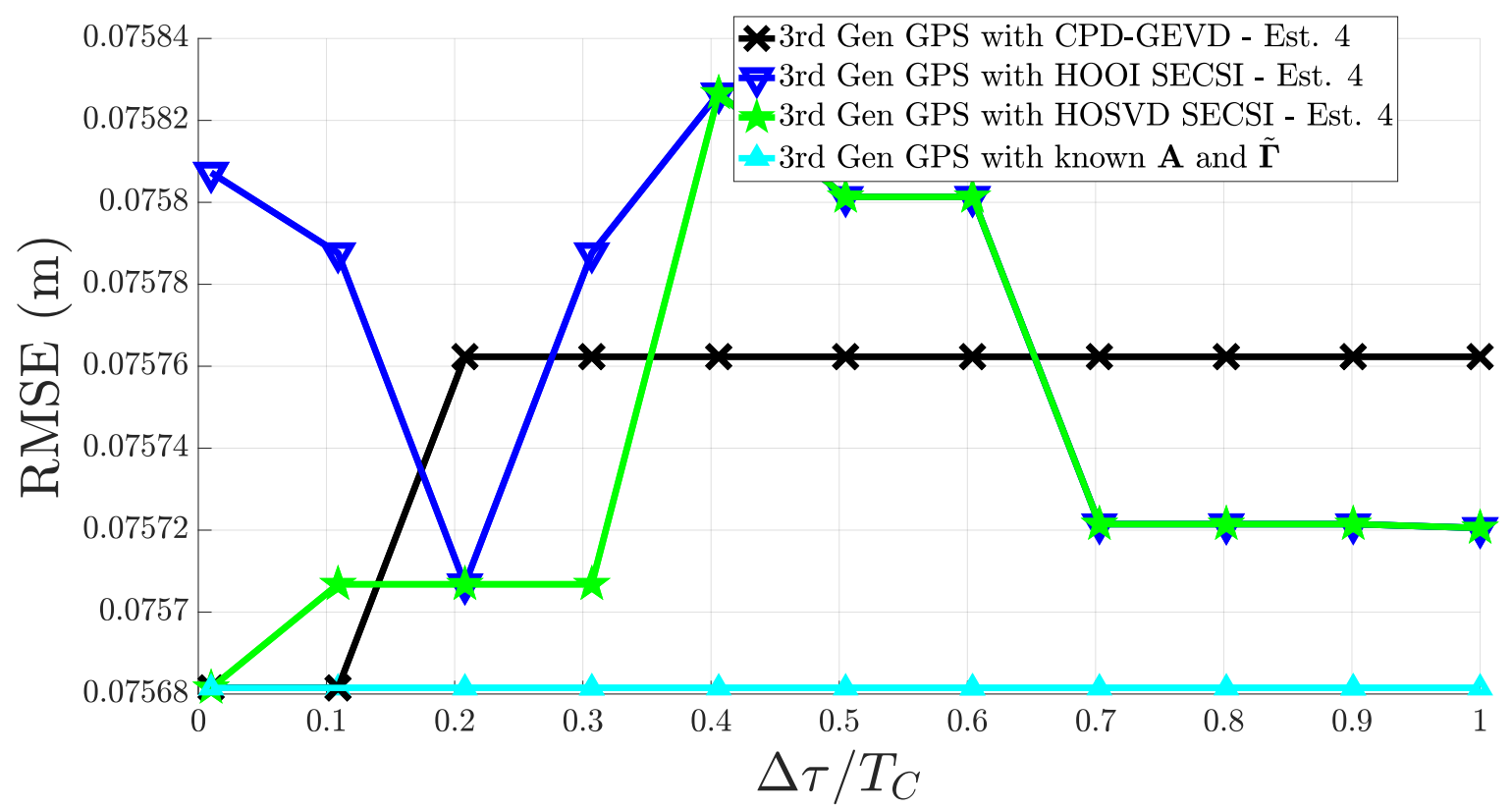

Figure 12: Simulação utilizando quarto fator estimado da técnica do estado-da-arte CPDGEVD, método HOSVD SECSI e método SECSI para GPS de terceira geração utilizando cada fator estimado com $M=8$ elementos e $L_{d}=2$ sinais recebidos. Em ambos os casos as amostras são coletadas por $K=30$ períodos que possuem $N=245520$ amostras. 
Já na Figura 13 é feita a comparação do método CPD-GEVD do estado-da-arte com o método HOSVD SECSI e HOOI SECSI utilizando o quinto fator estimado.

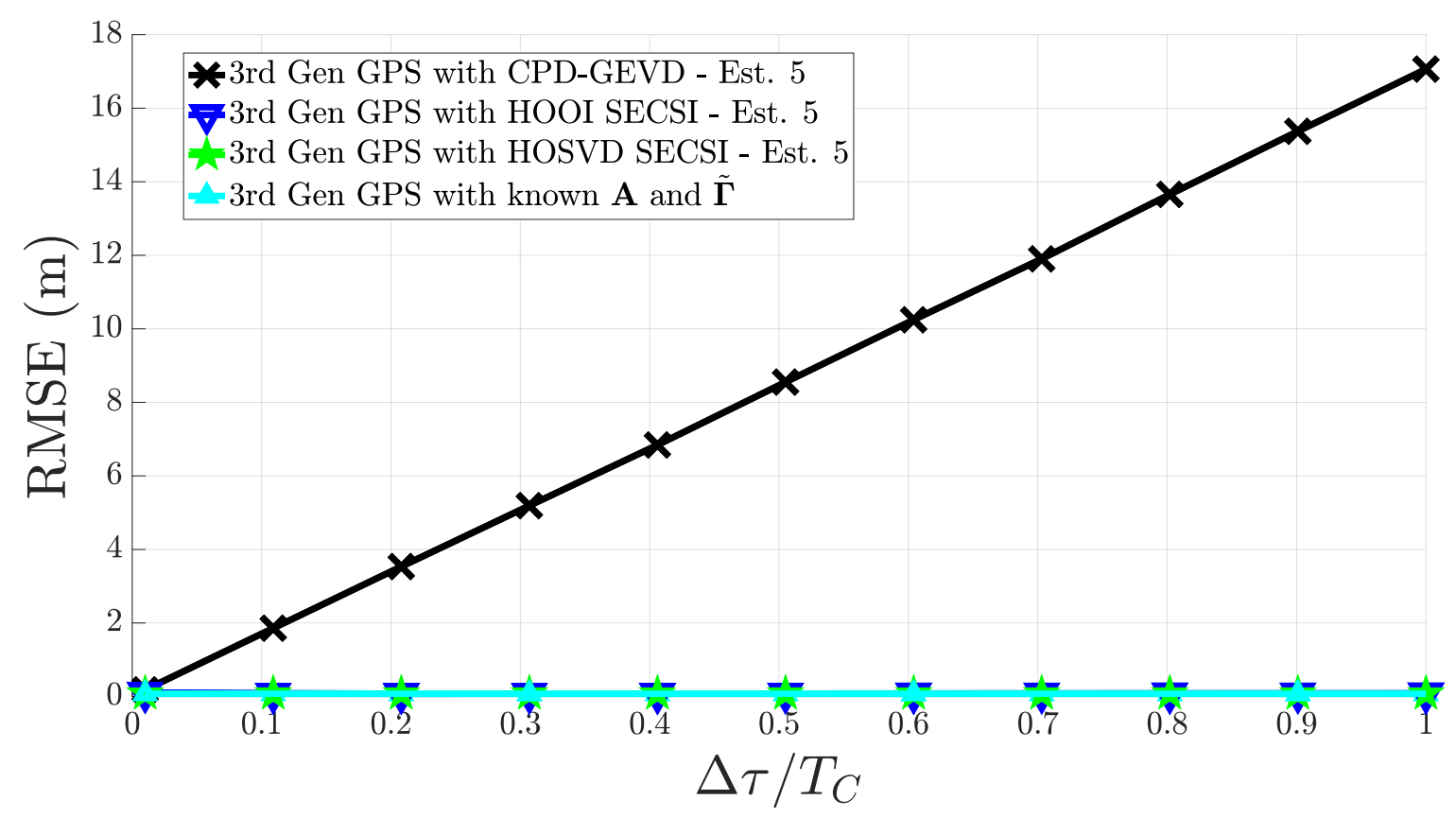

Figure 13: Simulação utilizando quinto fator estimado da técnica do estado-da-arte CPDGEVD, método HOSVD SECSI e método SECSI para GPS de terceira geração utilizando cada fator estimado com $M=8$ elementos e $L_{d}=2$ sinais recebidos. Em ambos os casos as amostras são coletadas por $K=30$ períodos que possuem $N=245520$ amostras.

Figura 14 exibe a comparação do método CPD-GEVD do estado-da-arte com o método HOSVD SECSI e HOOI SECSI utilizando o sexto fator estimado. 


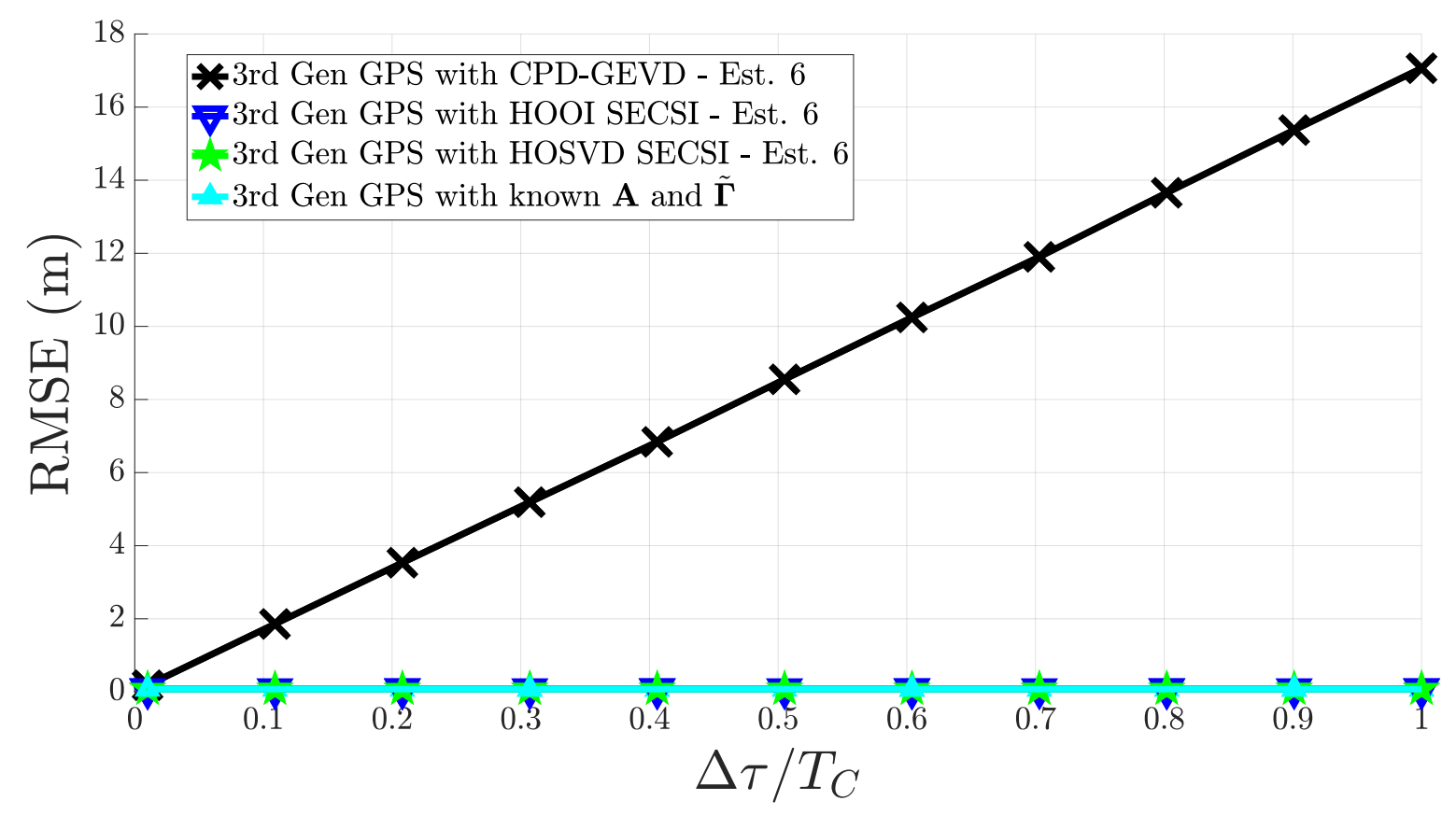

Figure 14: Simulação utilizando sexto fator estimado da técnica do estado-da-arte CPDGEVD, método HOSVD SECSI e método SECSI para GPS de terceira geração utilizando cada fator estimado com $M=8$ elementos e $L_{d}=2$ sinais recebidos. Em ambos os casos as amostras são coletadas por $K=30$ períodos que possuem $N=245520$ amostras.

Como mostrado nas Figuras 9 à 14 o primeiro e terceiro estimador de fatores para CPDGEVD apresentam os melhores resultado, dessa forma decide-se manter o uso CPD-GEVD do estado da arte. Da mesma forma, é possível notar que o primeiro e terceiro estimador de fatores para SECSI apresentam os melhores resultados, assim o primeiro estimador de fatores foi escolhido para realizar as demais comparações. Na Figura 15 é realizada a comparação das técnicas do estado-da-arte com o método proposto para GPS de segunda geração quando recebendo $L_{d}=2$ sinais. Note que os métodos DoA/KRF, CPD-GEVD e o HOSVD SECSI consideravelmente superam a técnica HOSVD+FBA+ESPS. Onde HOSVD+FBA+ESPS apresenta um erro máximo de aproximadamente $0.12 \mathrm{~m}$ enquanto as demais técnicas apresentam um erro de aproximadamente de $0.075 \mathrm{~m}$. 


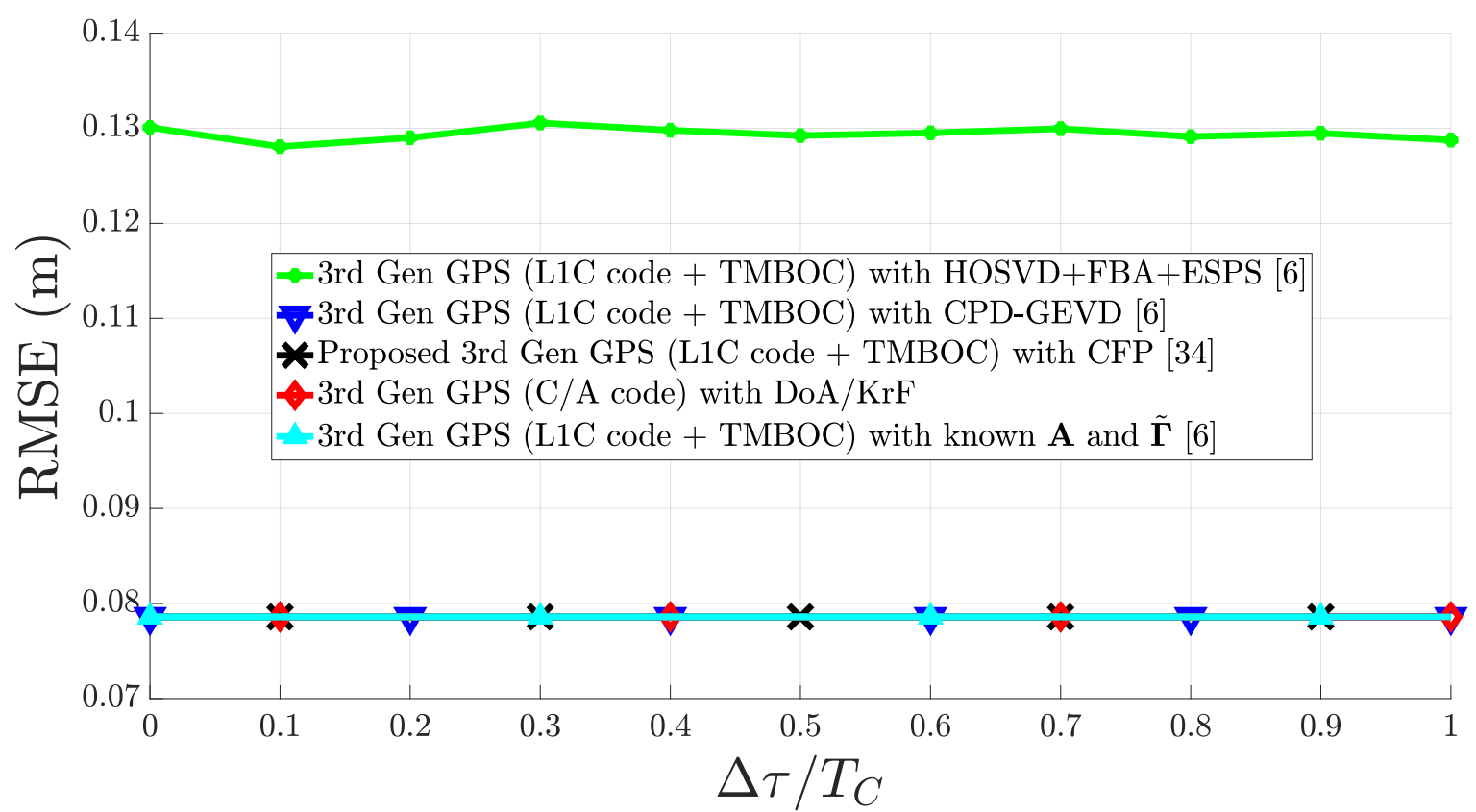

Figure 15: Técnicas do estado-da-arte e método SECSI proposto para GPS de terceira geração com $L_{d}=2$ sinais recebidos com $N=245520$ amostras coletadas durante $K=30$ utilizando $M=8$ antenas.

Já na Figura 16 é feita a comparação do método HOSVD+FBA+ESPS, DoA/KRF, e CPD-GEVD do estado-da-arte com o método SECSI proposto quando $L_{d}=3$. Observe que novamente as técnicas DoA/KRF, CPD-GEVD e o HOSVD SECSI superam o HOSVD+FBA+ESPS que apresenta um erro máximo de $0.13 \mathrm{~m}$. Ademais, note que o método CPD-GEVD apresenta pior performance quando $\Delta \tau<0.1 T_{c}$. Esse erro ocorre pela forte correlação entre as os sinais LOS e NLOS o que ocasiona um tensor de posto deficiente. Também, note que a técnica SECSI apresenta um pico quando os sinais estão fortemente correlatados, porém SECSI mostra-se mais tolerante a deficiência do posto e consegue estimar o atraso com sucesso. Além disso, ainda é possível notar que o método CPD-GEVD obtêm um melhor resultado quando combinado com o GPS de terceira geração. O melhor resultado obtido pelo GPS de terceira geração é atribuído ao uso da modulação TMBOC, uma vez que esta modulação permite melhor performance em cenário com multipercurso e aplica uma melhor separação espacial aos sinais. 


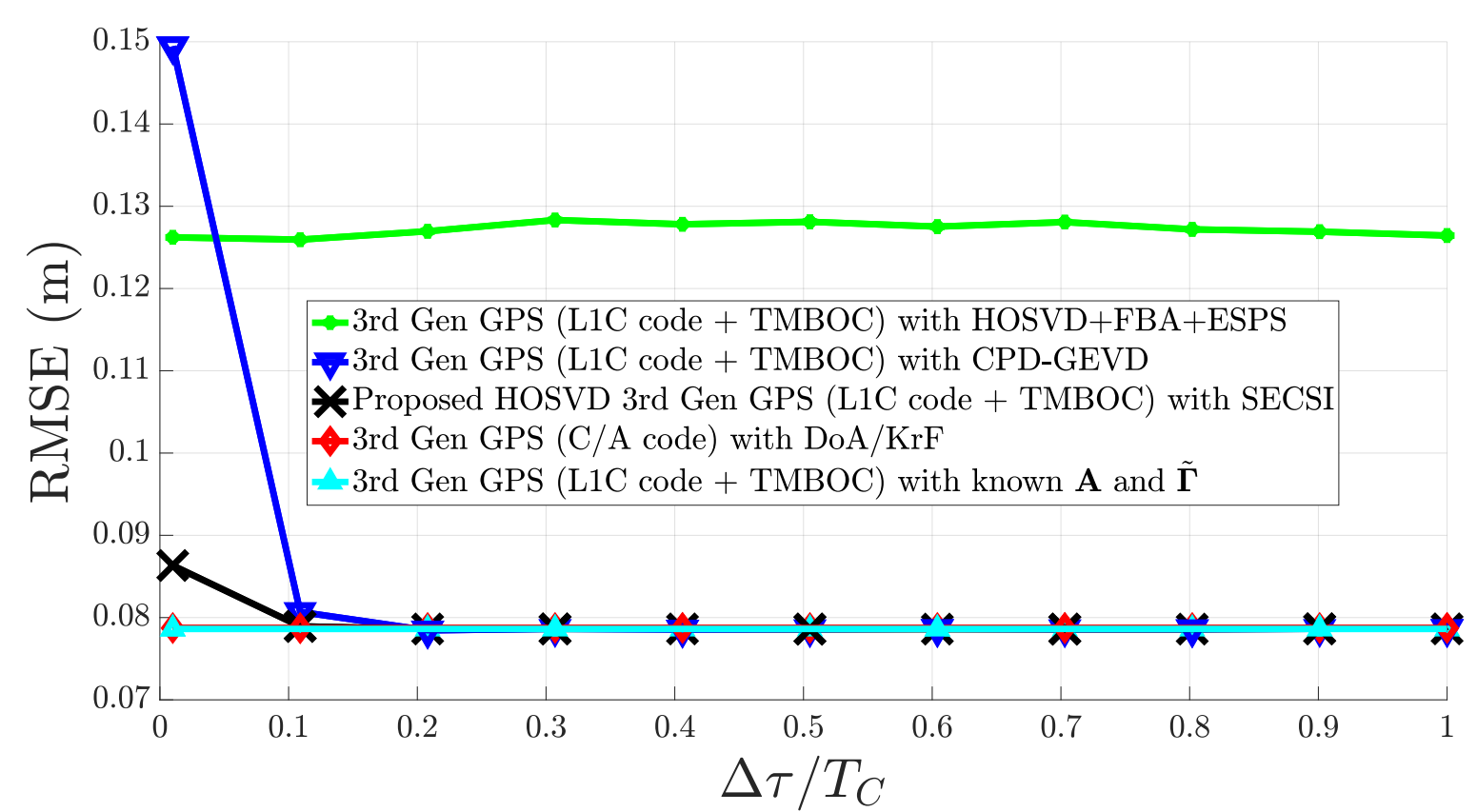

Figure 16: Técnicas do estado-da-arte e método SECSI proposto para GPS de terceira geração com $L_{d}=3$ sinais recebidos com $N=245520$ amostras coletadas durante $K=30$ utilizando $M=8$ antenas.

Na Figure 17 o método SECSI proposta é comparado com a substituição do HOSVD pelo HOOI quando $L_{d}=2$ sinais são recebidos. Percebe-se que tanto o uso de HOSVD como HOOI resultam em um erro muito similar para ambos GPS de segunda e terceira geração. É possível perceber que a segunda geração apresenta um erro máximo de 0.079 enquanto a terceira geração apresenta um erro máximo de 0.075. Ademais, SECSI com HOSVD supera levemente o método SECSI com HOOI quando LOS e NLOS estão correlatadas.

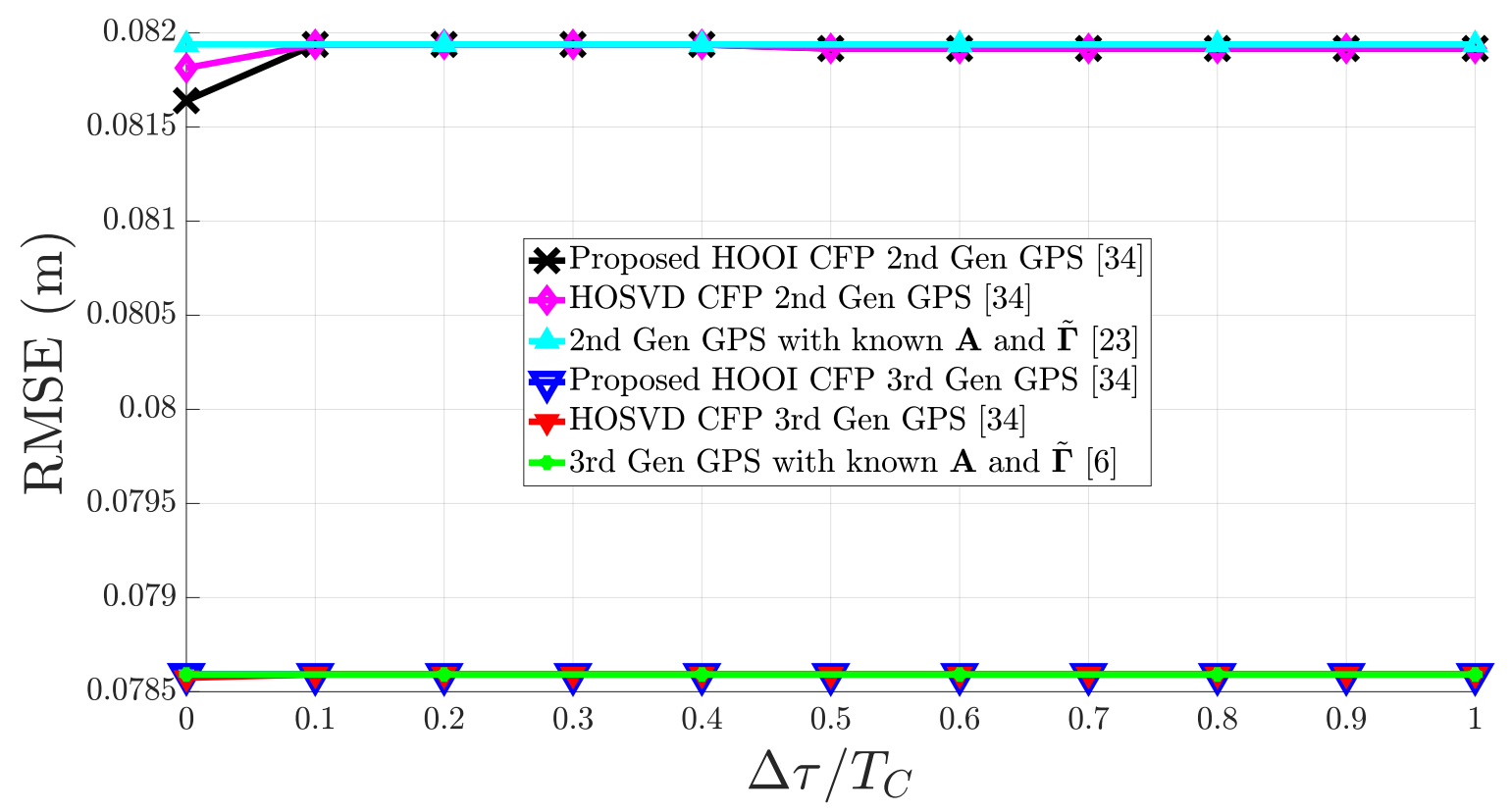

Figure 17: Método SECSI proposto com $L_{d}=2$ sinais ambos da segunda e terceira geração com $N=245520$ amostras coletadas durante $K=30$ utilizando $M=8$ antenas. 
Na Figure 18 o método SECSI proposta é comparado com a substituição da HOSVD pelo HOOI quando $L_{d}=3$ sinais recebidos. Novamente, percebe-se que tanto o uso de HOSVD como HOOI resultam em um erro muito similar para ambos GPS de segunda e terceira geração. É possível perceber que a segunda geração apresenta um erro máximo de 0.09 enquanto a terceira geração apresenta um erro máximo de 0.08. Ademais, SECSI com HOSVD supera levemente o método SECSI com HOOI quando LOS e NLOS estão correlatadas.

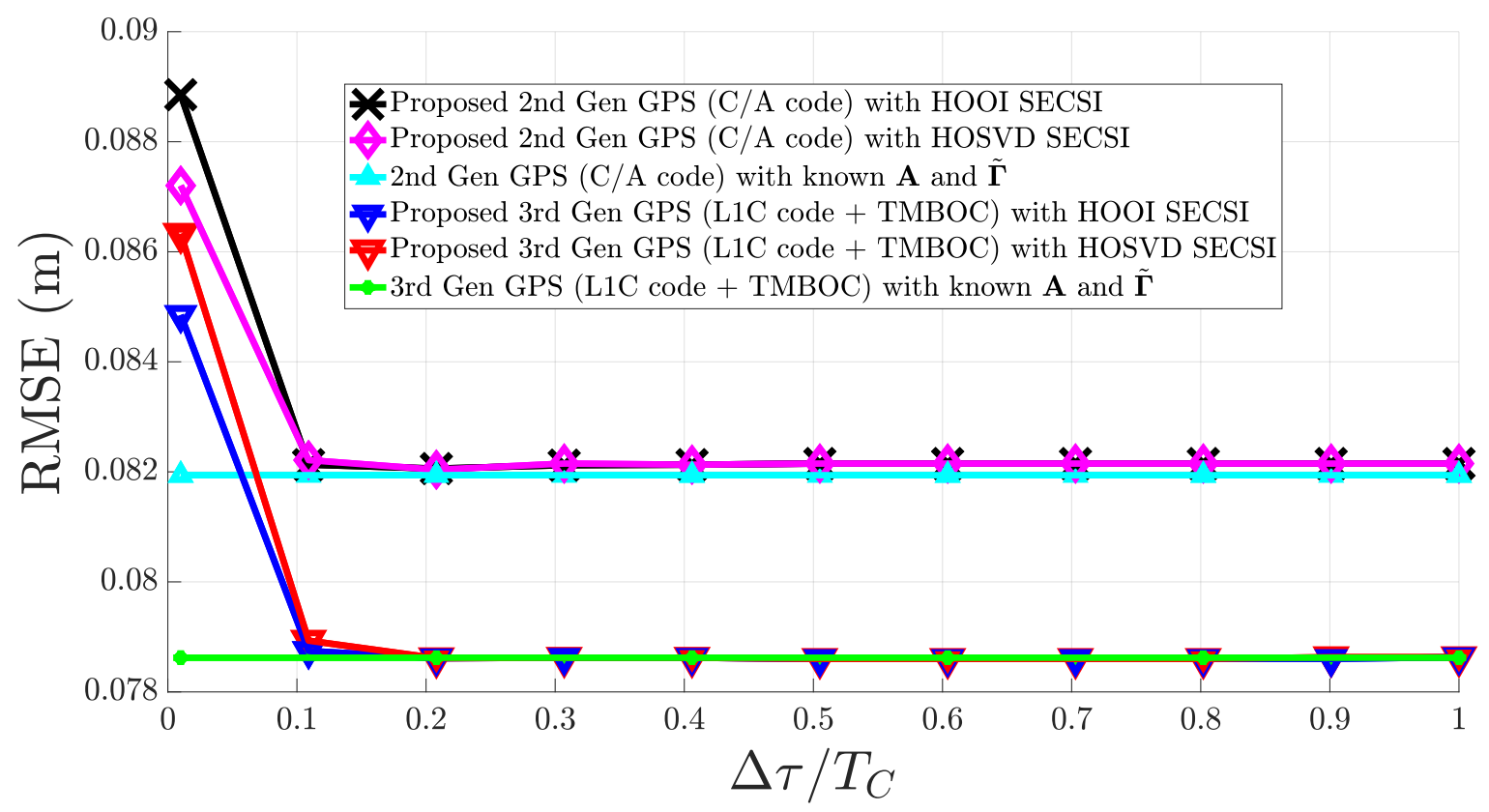

Figure 18: Simulação dos esquemas tensoriais do estado-da-arte e do método SECSI proposto com $L_{d}=3$ sinais ambos da segunda e terceira geração com $N=245520$ amostras coletadas durante $K=30$ utilizando $M=8$ antenas

\section{Arranjo de Antenas com Erro}

Nesta seção apresentamos o resultado das simulações utilizando os métodos de estimação de atraso do estado-da-arte para GPS de segunda e terceira geração e o método proposto HOSVD SECSI e HOOI SECSI. Supõe-se um arranjo de antenas com erros de posicionamento que recebem $L_{d}=2 \mathrm{e} L_{d}=3$ sinais com um atraso relativo fixado em $\Delta \tau=0.5 T_{c}$. Como foi decidido somente utilizar o primeiro fator estimado para os métodos CPD-GEVD e SECSI, neste cenário somente utilizamos este caso.

Na Figura 19 mostramos os resultados para a segunda geração quando o arranjo de antenas com erros recebe $L_{d}=2$ sinais. Nota-se que os métodos do estado-da-arte HOSVD+FBA+ESPS e DoA/KRF são sensíveis as imperfeições adicionadas ao arranjo de antenas. Entretanto, o método CPD-GEVD, o método HOSVD SECSI proposto e o HOOI SECSI mostram performance similar ao caso ideal. 


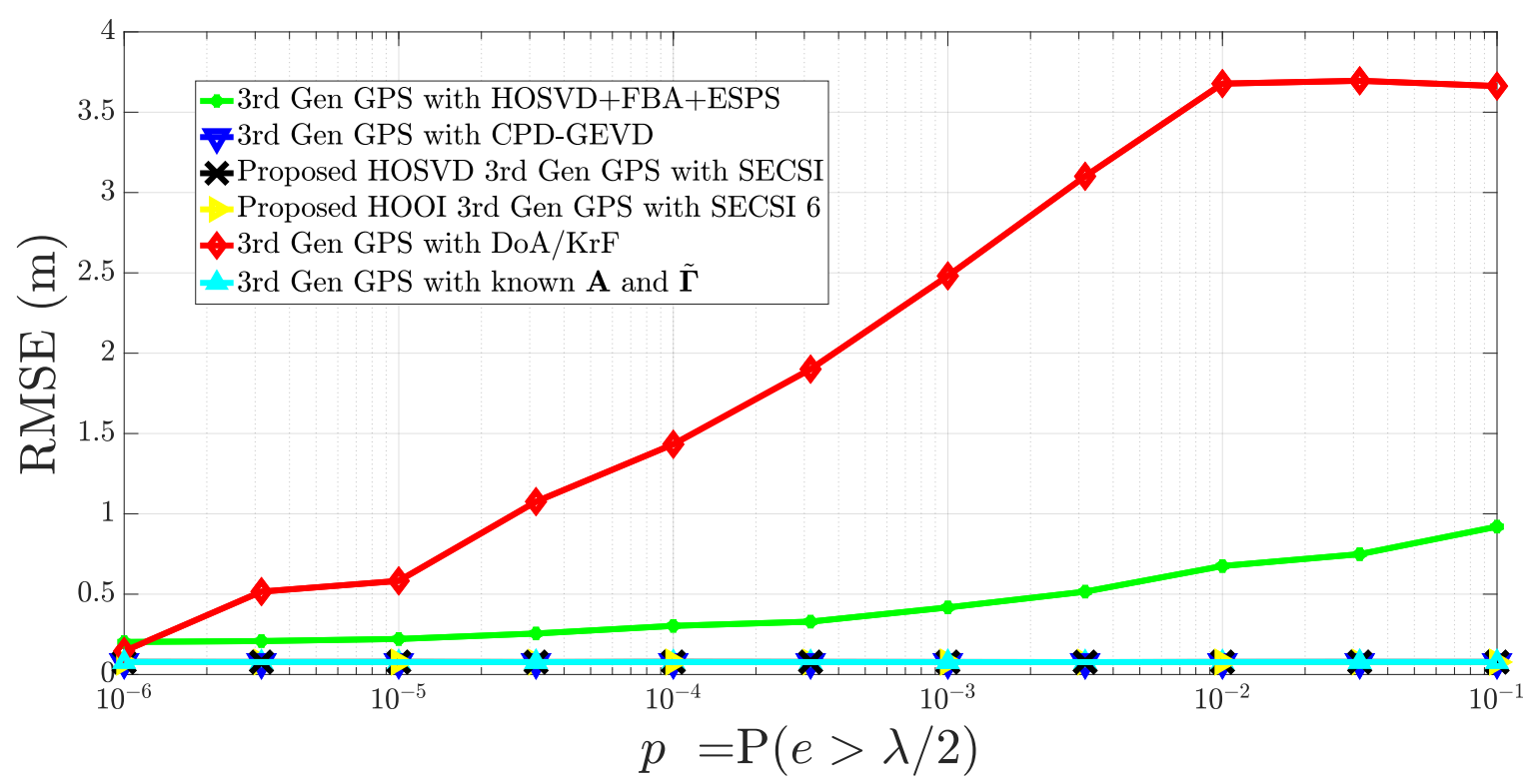

Figure 20: Simulação dos esquemas tensoriais do estado-da-arte e do método SECSI proposto com $L_{d}=2$ sinais da terceira geração com $N=245520$ amostras coletadas durante $K=30$ utilizando $M=8$ antenas

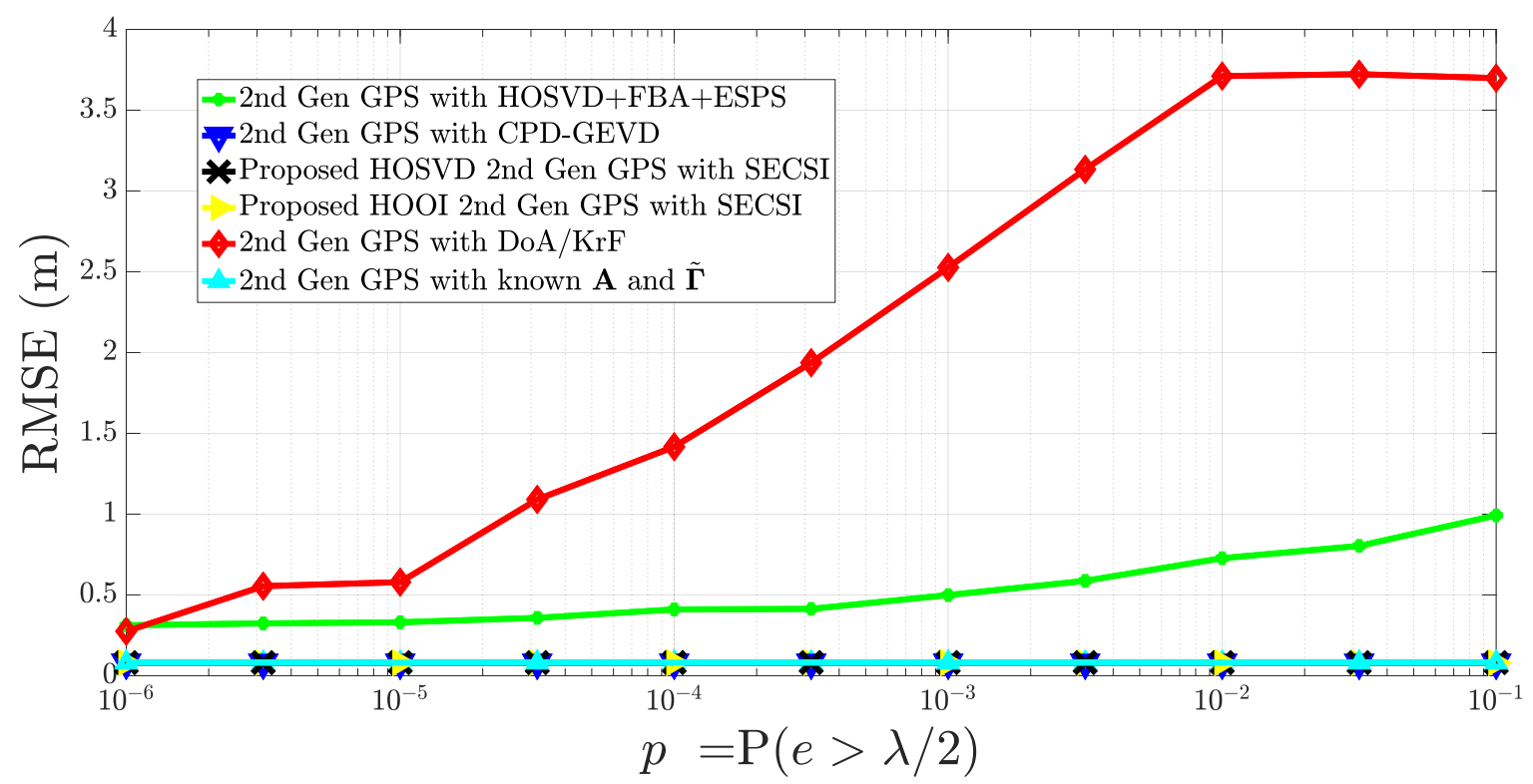

Figure 19: Simulação dos esquemas tensoriais do estado-da-arte e do método SECSI proposto com $L_{d}=2$ sinais da segunda geração com $N=245520$ amostras coletadas durante $K=30$ utilizando $M=8$ antenas

Na Figura 20 mostramos o resultado para GPS de terceira geração quando o arranjo de antenas com erros recebe $L_{d}=2$ sinais. Nesta figura, pode ser visto que mesmo utilizando o GPS de terceira geração os métodos do estado-da-arte HOSVD+FBA+ESPS e DoA/KRF são sensíveis as imperfeições do arranjo de antena. Inclusive, é possível notar que a terceira geração não trás um ganho significativo quando comparada com o GPS de segunda geração. Entretanto, o método CPD-GEVD, o método HOSVD SECSI proposto e o HOOI SECSI tem performance similar ao caso ideal. 


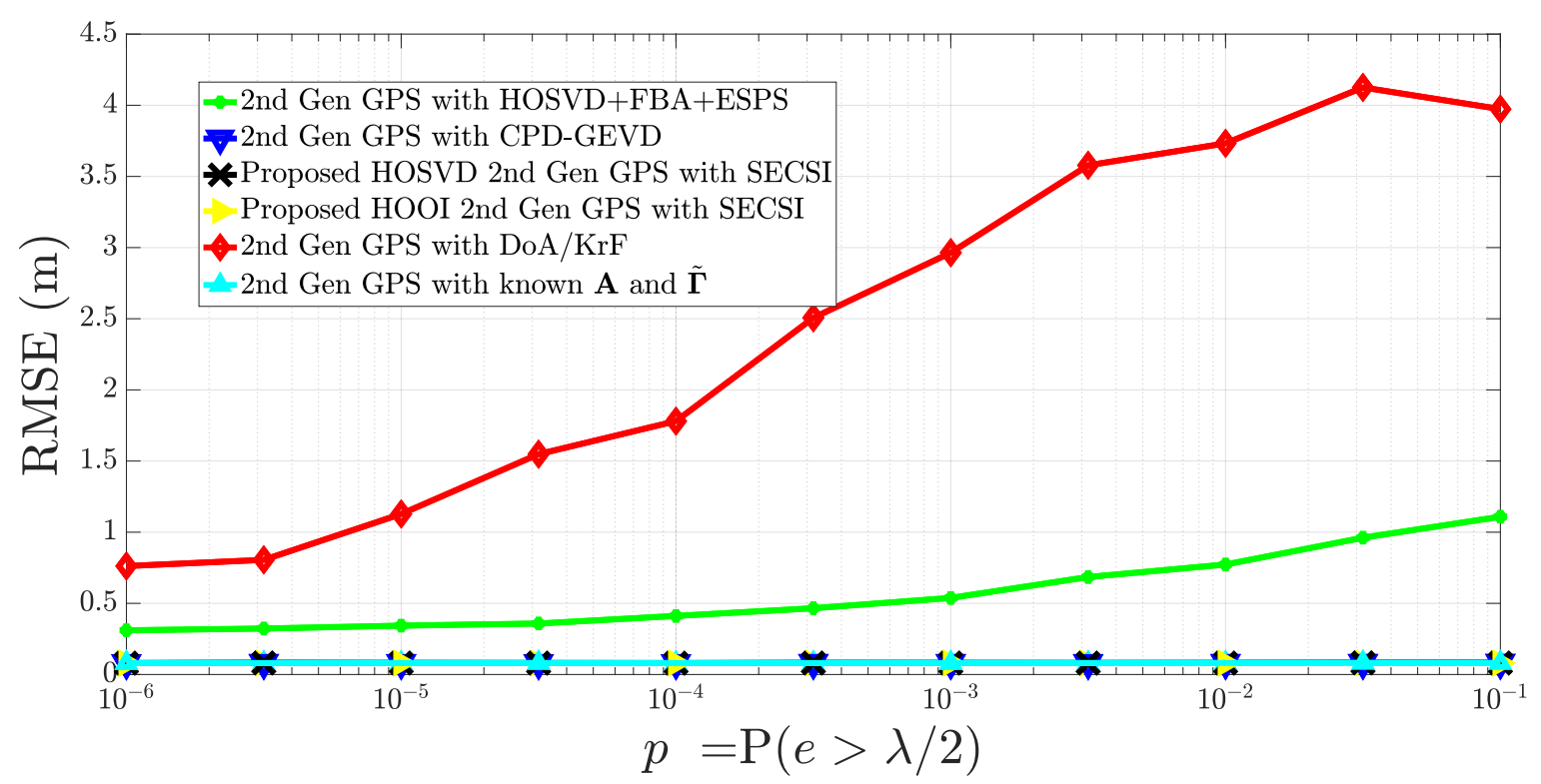

Figure 21: Simulação dos esquemas tensoriais do estado-da-arte e do método SECSI proposto com $L_{d}=3$ sinais da segunda geração com $N=245520$ amostras coletadas durante $K=30$ utilizando $M=8$ antenas.

Na Figura 21 temos o resultado para o GPS de segunda geração ao receber $L_{d}=3$ sinais. Nota-se que ao adicionar um componente NLOS se tem um aumento no erro da estimação do atraso quando utilizando métodos do estado-da-arte HOSVD+FBA+ESPS e DoA/KRF. Adicionalmente, é possível notar que o método CPD-GEVD, o método HOSVD SECSI proposto e o HOOI SECSI tem performance similar ao caso ideal.

Na Figura 22 mostramos os resultados para o GPS de terceira geração quando o arranjo de antenas com erros recebe $L_{d}=3$ sinais. Novamente, podemos ver que ao adicionar mais um componente NLOS se tem um aumento no erro da estimação do atraso quando utilizando estado-da-arte HOSVD+FBA+ESPS e DoA/KRF. Entretanto, pode-se notar que a adição do componente NLOS tem um baixo impacto na estimação do atraso uma vez que o HOSVD+FBA+ESPS tem um aumento de $0.1 \mathrm{~m}$ e o DoA/KRF mantêm quase que o mesmo erro. Mais uma vez, o método CPD-GEVD, o método HOSVD SECSI proposto e o HOOI SECSI tem performance similar ao caso ideal.

\section{Sinais Recebidos com DoA Variável}

Nesta seção apresentamos o resultado de simulações para os métodos do estado-da-arte e para o método HOSVD SECSI proposto para ambos GPS de segunda e terceira geração. Novamente supõe-se dois cenários em que um arranjo de antenas pode receber $L_{d}=2 \mathrm{e}$ $L_{d}=3$ sinais com um atraso relativo fixado em $\Delta \tau=0.5 T_{c}$. Quando temos $L_{d}=2$ sinais recebidos, definimos que o primeiro componente NLOS com um ângulo de chegada $\phi_{\mathrm{NLOS}_{1}}=\phi_{\mathrm{LOS}}+\Delta \phi$ e quando temos $L_{d}=3$ componentes de sinais, definimos o ângulo de chegada para a segunda NLOS como $\phi_{\mathrm{NLOS}_{2}}=\phi_{\mathrm{NLOS}_{1}}+\Delta \phi$. Em ambos os cenários 


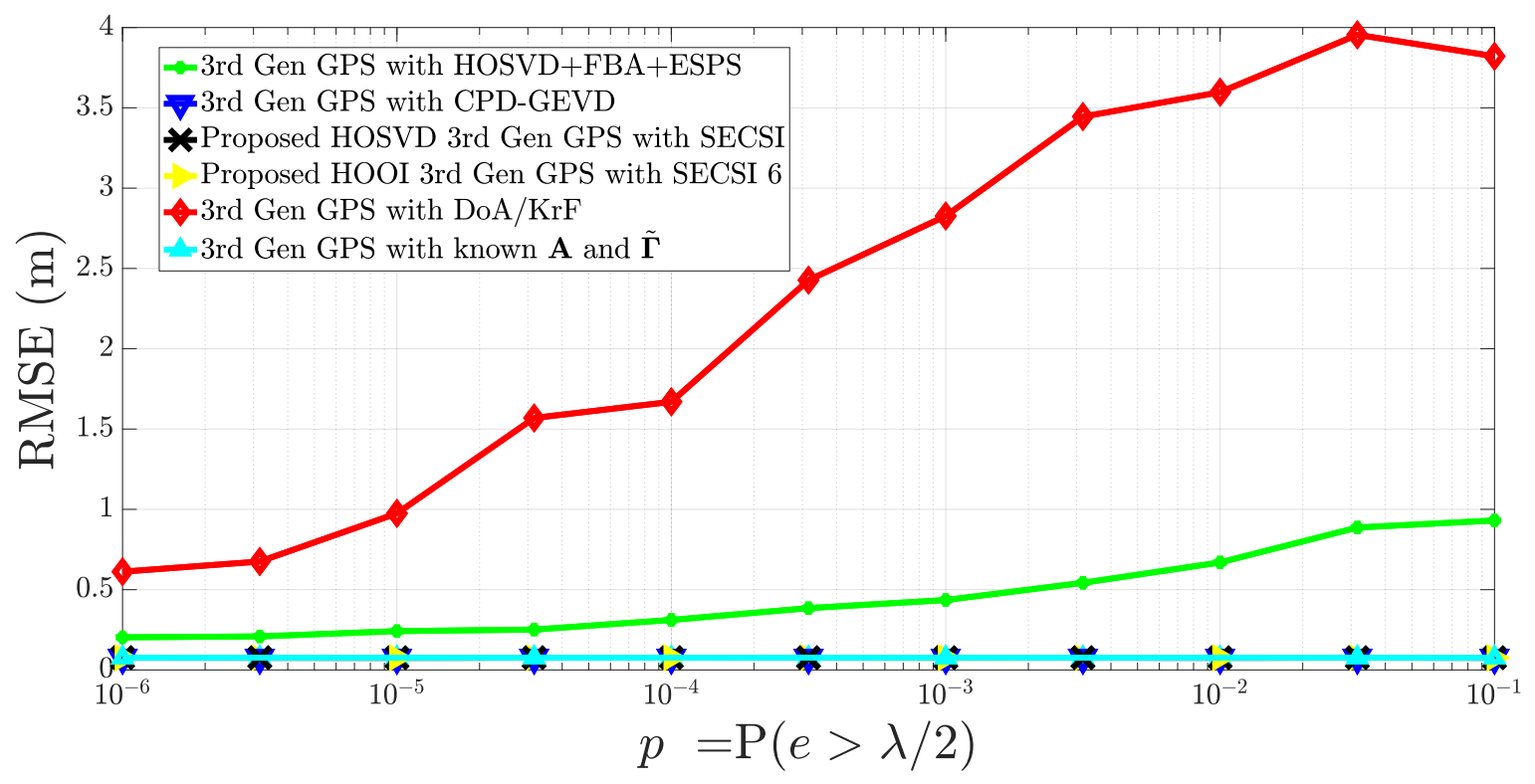

Figure 22: Simulação dos esquemas tensoriais do estado-da-arte e do método SECSI proposto com $L_{d}=3$ sinais da terceira geração com $N=245520$ amostras coletadas durante $K=30$ utilizando $M=8$ antenas

supõe-se que utilizamos ângulos randômicos que variam entre $-0.25 \mathrm{rad}$ e $0.25 \mathrm{rad}$ para o componente LOS. Assim, cada simulação irá variar o ângulo de chegada de cada NLOS baseado no ângulo de chegada do componente LOS. Dessa forma, pode-se identificar a menor diferença de ângulo de chegada de LOS para NLOS.

Na Figura 23 temos os resultado para o GPS de segunda geração para o cenário em que recebemos $L_{d}=2$ sinais e utilizamos o ângulo de chegada de LOS para calcular o ângulo de chegada de NLOS. Pode-se notar que os métodos do estado-da-arte HOSVD+FBA+ESP e o DoA/KRF são sensíveis à diferença de ângulo de chegada. Também, podemos notar que o método estado-da-arte CPD-GEVD e o método proposto HOSVD SECSI tem um erro maior do que o DoA/KRF quando temos $\Delta \phi=0 \times 0.25 \mathrm{rad}$. Entretanto, o método estado-daarte CPD-GEVD e o método proposto HOSVD SECSI tem melhor performance do que o DoA/KRF quando $\Delta \phi=0.0025 \times 0.25 \mathrm{rad}$. 


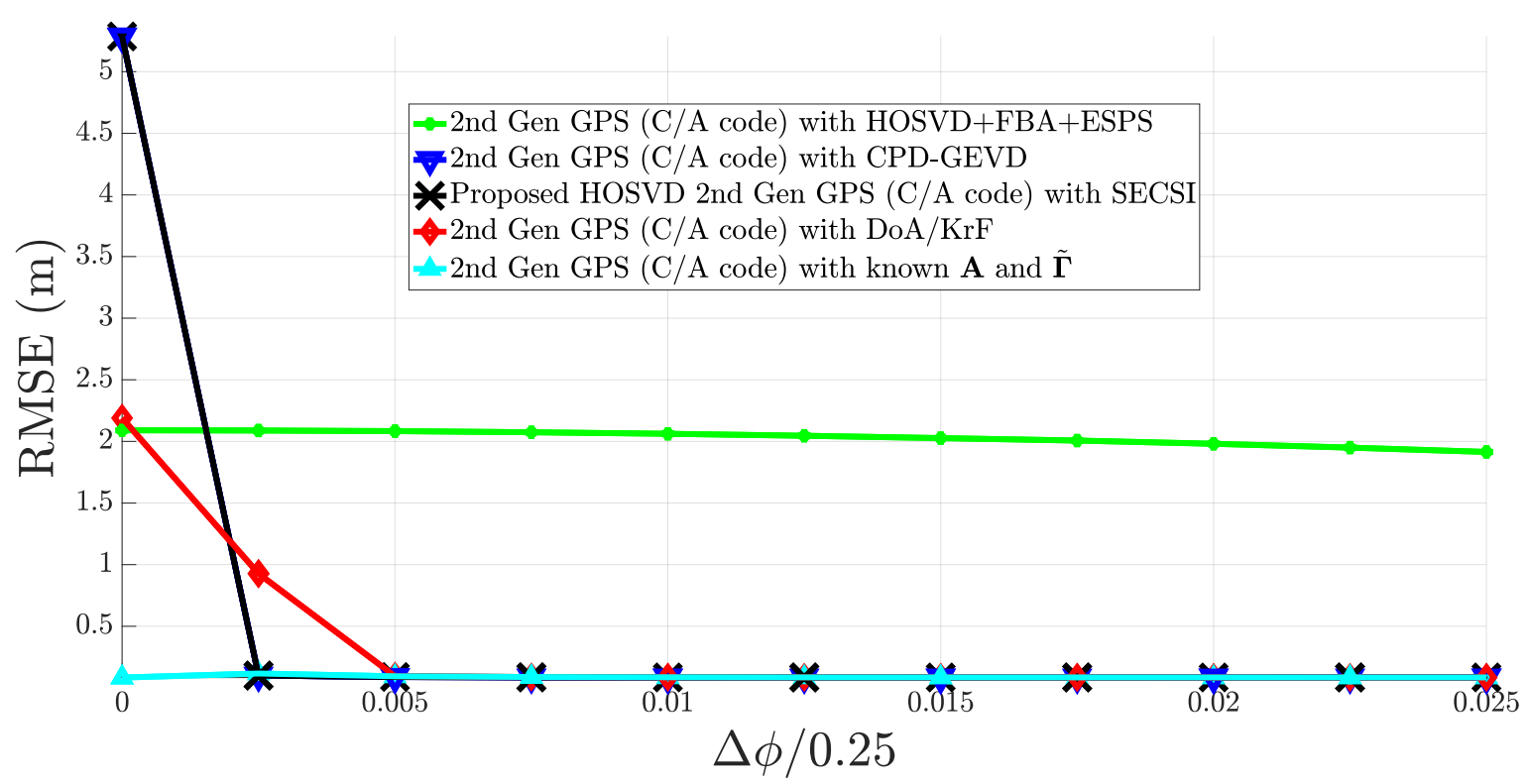

Figure 23: Simulação dos esquemas tensoriais do estado-da-arte e do método SECSI proposto com $L_{d}=2$ sinais da segunda geração com $N=245520$ amostras coletadas durante $K=30$ utilizando $M=8$ antenas

Na Figura 24 mostramos o resultado para GPS de segunda geração quando temos $L_{d}=3$ sinais. Mostramos que os métodos do estado-da-arte HOSVD+FBA+ESP e o DoA/KRF são sensíveis à diferença de ângulo de chegada. Entretanto, podemos notar que o método estado-da-arte CPD-GEVD e o método proposto HOSVD SECSI tem um erro maior do que o DoA/KRF quando temos $\Delta \phi=0 \times 0.25 \mathrm{rad}$. Também é possível notar que método estadoda-arte CPD-GEVD e o método proposto HOSVD SECSI tem melhor performance do que o DoA/KRF quando $\Delta \phi=0.0025 \times 0.25 \mathrm{rad}$. Além disso, o método HOSVD SECSI proposto apresenta melhor performance do que o método CPD-GEVD quando temos somente uma diferença de ângulo de chegada $\Delta \phi=0 \times 0.25 \mathrm{rad}$. 


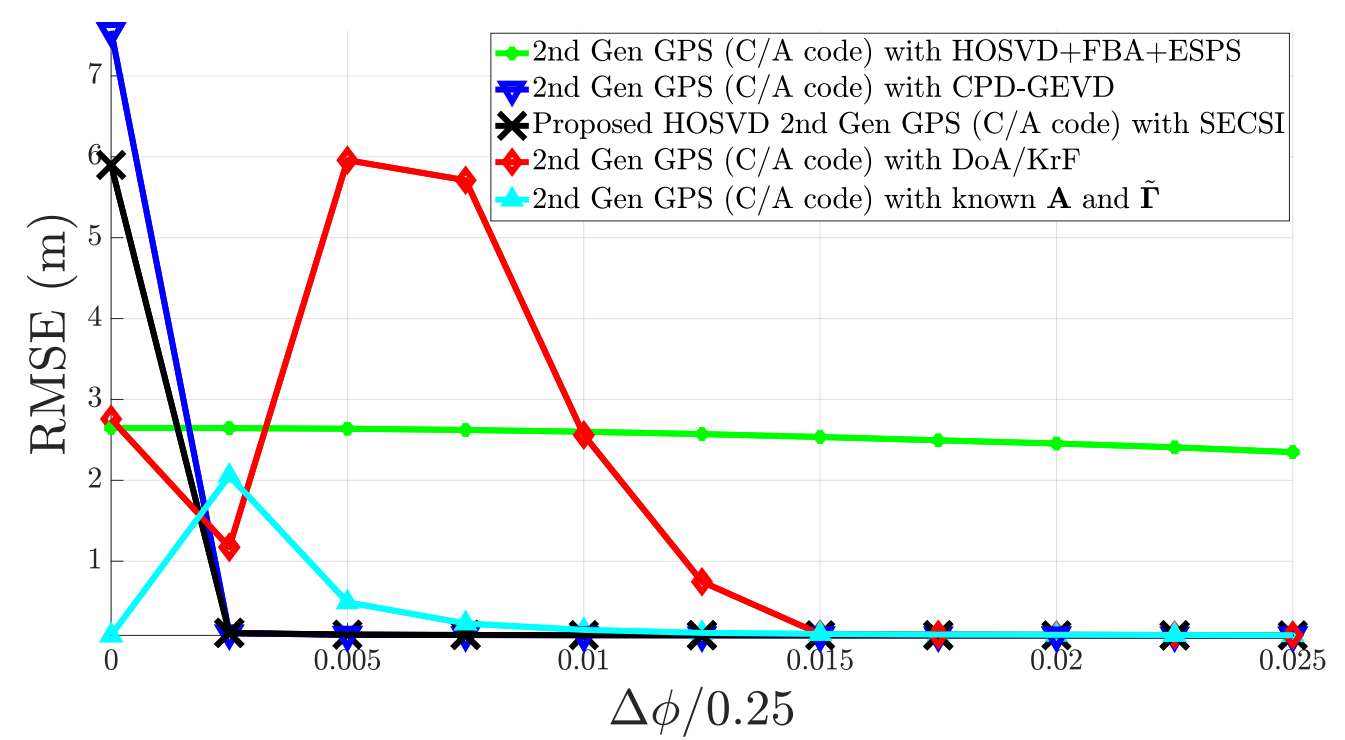

Figure 24: Simulação dos esquemas tensoriais do estado-da-arte e do método SECSI proposto com $L_{d}=3$ sinais da segunda geração com $N=245520$ amostras coletadas durante $K=30$ utilizando $M=8$ antenas

Na Figura 25 temos os resultado para o GPS de terceira geração quando consideramos que o arranjo de antenas recebe $L_{d}=2$ sinais. Novamente mostramos que os métodos do estado-da-arte HOSVD+FBA+ESP e o DoA/KRF são sensíveis à diferença de ângulo de chegada. Mais uma vez pode-se notar que o método estado-da-arte CPD-GEVD e o método proposto HOSVD SECSI apresentam um maior erro do que o DoA/KRF quando $\Delta \phi=$ $0 \times 0.25$ rad. Adicionalmente, nota-se que o GPS de terceira geração apresenta uma pequena melhora na estimação de atraso.

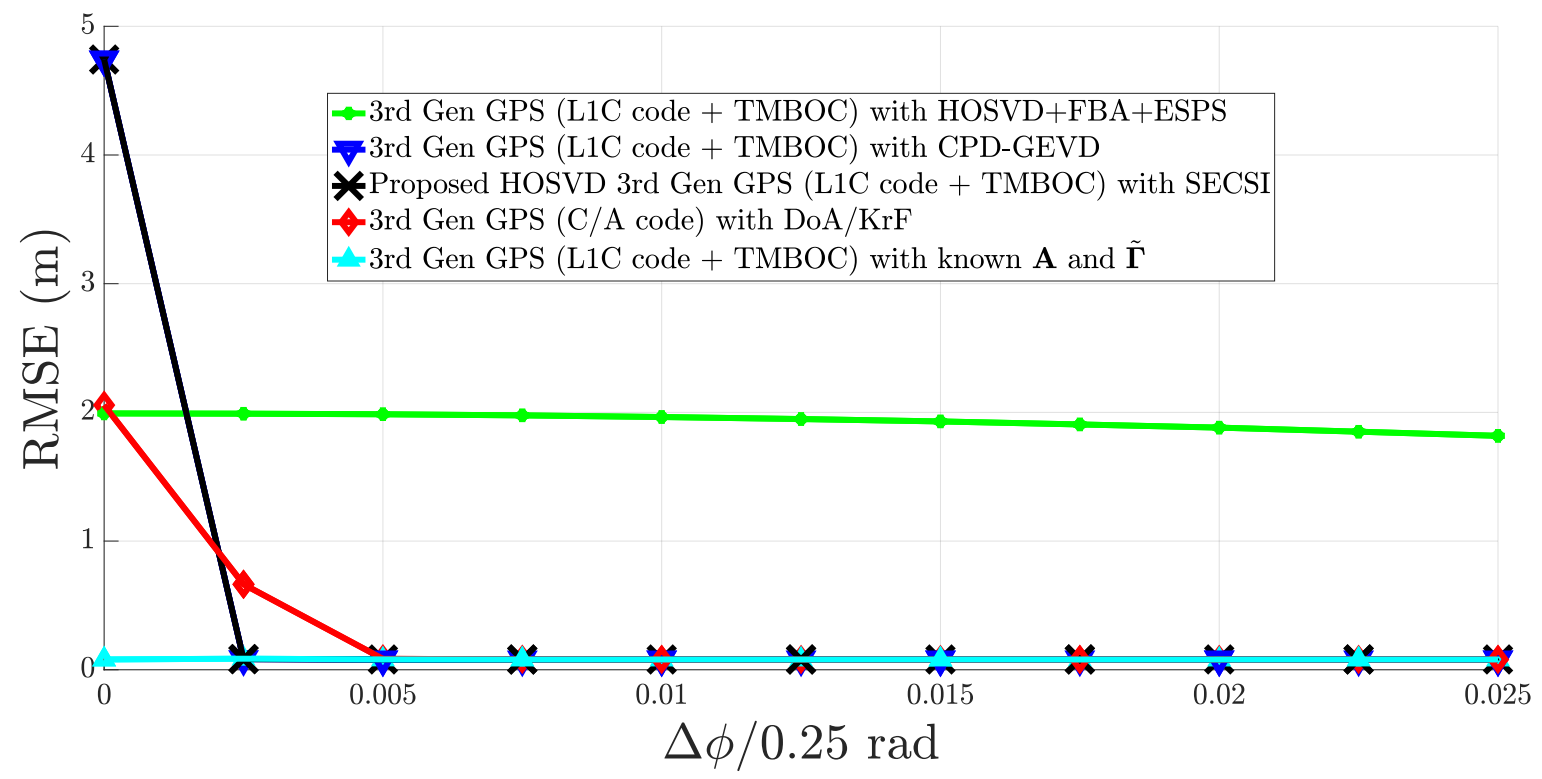

Figure 25: Simulação dos esquemas tensoriais do estado-da-arte e do método SECSI proposto com $L_{d}=2$ sinais da terceira geração com $N=245520$ amostras coletadas durante $K=30$ utilizando $M=8$ antenas 
Na Figura 26 mostramos os resultados da simulação quando recebendo $L_{d}=3$ sinais do GPS de terceira geração. Novamente, nota-se que os métodos do estado-da-arte HOSVD+FBA+ESP e o DoA/KRF são sensíveis à diferença de ângulo de chegada. Mais uma vez pode-se notar que o método estado-da-arte CPD-GEVD e o método proposto HOSVD SECSI apresentam um maior erro do que o DoA/KRF quando $\Delta \phi=0 \times 0.25$ rad. Adicionalmente, é possível notar que o método HOSVD SECSI proposto e que o GPS de terceira geração apresentam um menor erro quando comparados com o GPS de segunda geração.

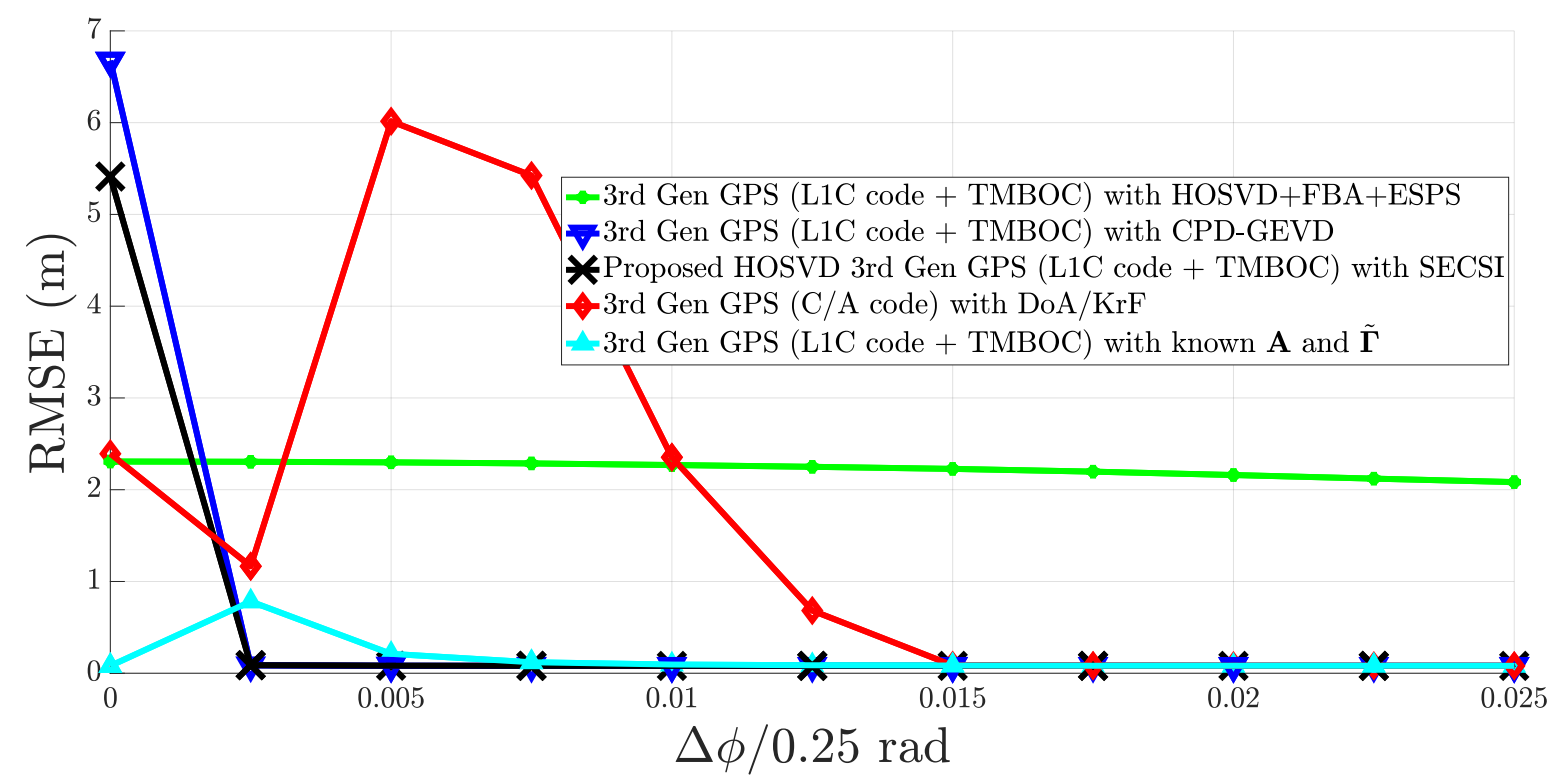

Figure 26: Simulação dos esquemas tensoriais do estado-da-arte e do método SECSI proposto com $L_{d}=3$ sinais da terceira geração com $N=245520$ amostras coletadas durante $K=30$ utilizando $M=8$ antenas 


\section{Sumário}

1 IntrodUÇão................................................ 1

2 Modelo de Dados $\ldots \ldots \ldots \ldots \ldots \ldots \ldots \ldots \ldots \ldots \ldots \ldots \ldots \ldots \ldots \ldots \ldots, 3$

2.1 Cenário do Modelo de Dados .................................... 4

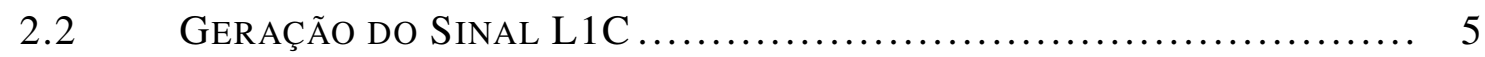

2.3 Modelo de Dados PrÉ-CorrelaÇão................................. 8

2.4 Modelo de Dados Pós-CorrelaÇão............................... 9

3 Estimação de Atraso baseado em Técnicas Tensoriais e SimulaÇõES.................................................. 10

3.1 Estado-DA-ARTe Para EstimaÇã De ATRaso Tensorial Para A GPS DE TERCEIRA GERAÇÃO ..................................... 10

3.1.1 ESTIMAÇÃo de ATRaso Baseado EM HOSVD ...................... 11

3.1.2 ESTIMAÇÃO DE ATRASO BASEAdo EM DoA/KRF .................... 12

3.1.2.1 EstimaÇão de ATRASO ......................................... 14

3.1.3 EstimaÇão de Atraso Baseado EM CPD-GEVD .................. 15

3.2 Proposta de Estrutura Semi-Algébrica para Decomposição Canonical Polyadic Aproximadas Através de DiagonalizaÇões Simultâneas Para EstimaÇão de Atraso .................... 19

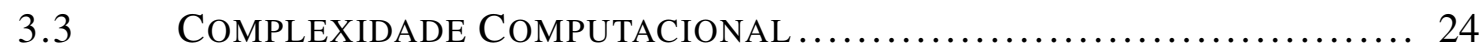

3.3.1 COMPLEXIDADE DO HOSVD+FBA+ESPS ...................... 24

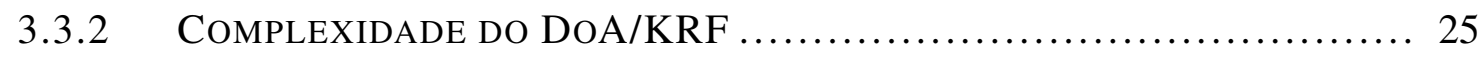

3.3.3 COMPLEXIDADE DO CPD-GEVD ................................ 26

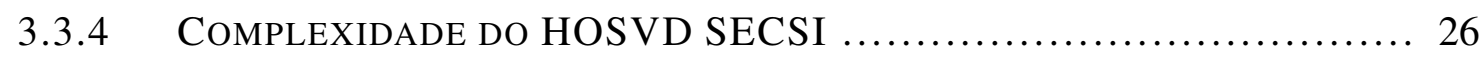

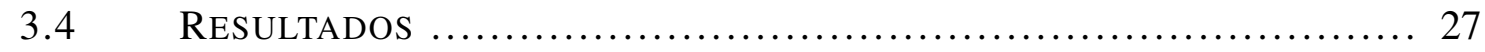

3.4.1 CenÁrio do ESTADO-DA-ARTE ................................... 27

3.4.2 CenÁRio Proposto .................................................. 29

3.4.2.1 EstimaÇão de Atraso Para GPS de Segunda GeraÇão........... 30

3.4.2.2 EstimaÇão de Atraso Para GPS de Terceira GeraÇão .......... 37

3.4.2.3 ARRANJO DE ANTENAS COM ERROS ............................. 46

3.4.2.4 Sinais Recebidos com Direção de Chegada VARiáVEl ........... 49

4 Conclusão.................................................. 53 
4.1 Trabalhos Futuros

4 REFERÊnCIAS Bibliográfichas......................... 54 


\section{LIST OF FIGURES}

1 Simulação utilizando o primeiro fator estimado da técnica do estado-da-arte CPD-GEVD, método HOSVD SECSI e método SECSI para GPS de segunda geração utilizando cada fator estimado com $M=8$ elementos e $L_{d}=2$ sinais recebidos. Em ambos os casos as amostras são coletadas por $K=30$ períodos que possuem $N=245520$ amostras. .................................xvii

2 Simulação utilizando o segundo fator estimado da técnica do estado-da-arte CPD-GEVD, método HOSVD SECSI e método SECSI para GPS de segunda geração utilizando cada fator estimado com $M=8$ elementos e $L_{d}=2$ sinais recebidos. Em ambos os casos as amostras são coletadas por $K=30$ períodos que possuem $N=245520$ amostras.

3 Simulação utilizando o terceiro fator estimado da técnica do estado-da-arte CPD-GEVD, método HOSVD SECSI e método SECSI para GPS de segunda geração utilizando cada fator estimado com $M=8$ elementos e $L_{d}=2$ sinais recebidos. Em ambos os casos as amostras são coletadas por $K=30$ períodos que possuem $N=245520$ amostras.

4 Simulação utilizando o quarto fator estimado da técnica do estado-da-arte CPD-GEVD, método HOSVD SECSI e método SECSI para GPS de segunda geração utilizando cada fator estimado com $M=8$ elementos e $L_{d}=2$ sinais recebidos. Em ambos os casos as amostras são coletadas por $K=30$ períodos que possuem $N=245520$ amostras.

5 Simulação utilizando o quinto fator estimado da técnica do estado-da-arte CPD-GEVD, método HOSVD SECSI e método SECSI para GPS de segunda geração utilizando cada fator estimado com $M=8$ elementos e $L_{d}=2$ sinais recebidos. Em ambos os casos as amostras são coletadas por $K=30$ períodos que possuem $N=245520$ amostras.

6 Simulação utilizando o sexto fator estimado da técnica do estado-da-arte CPD-GEVD, método HOSVD SECSI e método SECSI para GPS de segunda geração utilizando cada fator estimado com $M=8$ elementos e $L_{d}=2$ sinais recebidos. Em ambos os casos as amostras são coletadas por $K=30$ períodos que possuem $N=245520$ amostras. 
9 Simulação utilizando primeiro fator estimado da técnica do estado-da-arte CPD-GEVD, método HOSVD SECSI e método SECSI para GPS de terceira geração utilizando cada fator estimado com $M=8$ elementos e $L_{d}=2$ sinais recebidos. Em ambos os casos as amostras são coletadas por $K=30$ períodos que possuem $N=245520$ amostras.

10 Simulação utilizando segundo fator estimado da técnica do estado-da-arte CPD-GEVD, método HOSVD SECSI e método SECSI para GPS de terceira geração utilizando cada fator estimado com $M=8$ elementos e $L_{d}=2$ sinais recebidos. Em ambos os casos as amostras são coletadas por $K=30$ períodos que possuem $N=245520$ amostras.

11 Simulação utilizando terceiro fator estimado da técnica do estado-da-arte CPD-GEVD, método HOSVD SECSI e método SECSI para GPS de terceira geração utilizando cada fator estimado com $M=8$ elementos e $L_{d}=2$ sinais recebidos. Em ambos os casos as amostras são coletadas por $K=30$ períodos que possuem $N=245520$ amostras.

12 Simulação utilizando quarto fator estimado da técnica do estado-da-arte CPD-GEVD, método HOSVD SECSI e método SECSI para GPS de terceira geração utilizando cada fator estimado com $M=8$ elementos e $L_{d}=2$ sinais recebidos. Em ambos os casos as amostras são coletadas por $K=30$ períodos que possuem $N=245520$ amostras.

13 Simulação utilizando quinto fator estimado da técnica do estado-da-arte CPD-GEVD, método HOSVD SECSI e método SECSI para GPS de terceira geração utilizando cada fator estimado com $M=8$ elementos e $L_{d}=2$ sinais recebidos. Em ambos os casos as amostras são coletadas por $K=30$ períodos que possuem $N=245520$ amostras.

14 Simulação utilizando sexto fator estimado da técnica do estado-da-arte CPDGEVD, método HOSVD SECSI e método SECSI para GPS de terceira geração utilizando cada fator estimado com $M=8$ elementos e $L_{d}=2$ sinais recebidos. Em ambos os casos as amostras são coletadas por $K=30$ períodos que possuem $N=245520$ amostras.

15 Técnicas do estado-da-arte e método SECSI proposto para GPS de terceira geração com $L_{d}=2$ sinais recebidos com $N=245520$ amostras coletadas durante $K=30$ utilizando $M=8$ antenas. xxvii

16 Técnicas do estado-da-arte e método SECSI proposto para GPS de terceira geração com $L_{d}=3$ sinais recebidos com $N=245520$ amostras coletadas durante $K=30$ utilizando $M=8$ antenas. XXViii

20 Simulação dos esquemas tensoriais do estado-da-arte e do método SECSI proposto com $L_{d}=2$ sinais da terceira geração com $N=245520$ amostras coletadas durante $K=30$ utilizando $M=8$ antenas 
19 Simulação dos esquemas tensoriais do estado-da-arte e do método SECSI proposto com $L_{d}=2$ sinais da segunda geração com $N=245520$ amostras coletadas durante $K=30$ utilizando $M=8$ antenas

21 Simulação dos esquemas tensoriais do estado-da-arte e do método SECSI proposto com $L_{d}=3$ sinais da segunda geração com $N=245520$ amostras coletadas durante $K=30$ utilizando $M=8$ antenas.

22 Simulação dos esquemas tensoriais do estado-da-arte e do método SECSI proposto com $L_{d}=3$ sinais da terceira geração com $N=245520$ amostras coletadas durante $K=30$ utilizando $M=8$ antenas Xxxii

23 Simulação dos esquemas tensoriais do estado-da-arte e do método SECSI proposto com $L_{d}=2$ sinais da segunda geração com $N=245520$ amostras coletadas durante $K=30$ utilizando $M=8$ antenas XXXiii

24 Simulação dos esquemas tensoriais do estado-da-arte e do método SECSI proposto com $L_{d}=3$ sinais da segunda geração com $N=245520$ amostras coletadas durante $K=30$ utilizando $M=8$ antenas xxxiv

25 Simulação dos esquemas tensoriais do estado-da-arte e do método SECSI proposto com $L_{d}=2$ sinais da terceira geração com $N=245520$ amostras coletadas durante $K=30$ utilizando $M=8$ antenas xxxiv

26 Simulação dos esquemas tensoriais do estado-da-arte e do método SECSI proposto com $L_{d}=3$ sinais da terceira geração com $N=245520$ amostras coletadas durante $K=30$ utilizando $M=8$ antenas XXXV

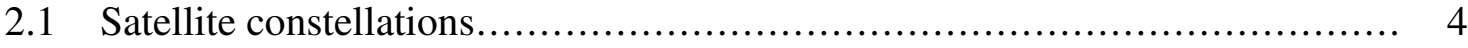

2.2 Receiver receiving one LOS e $L_{d}$ NLOS signals ............................. 4

2.3 Generation of the $\mathrm{L} 1 \mathrm{C}$ pilot code ........................................... 5

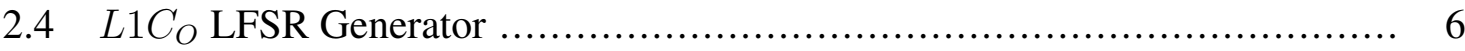

2.5 L1C pilot code and $L 1 C_{O}$ code time relationship ............................ 7

2.6 L1C pilot with TMBOC modulation......................................... 8

3.1 HOSVD Time-Delay Estimation block diagram............................ 12

$3.2 \mathrm{DoA} / \mathrm{KrF}$ Time-delay estimation block ................................... 14

3.3 CPD-GEVD Time-Delay Estimation block diagram ........................... 18

3.4 Proposed SECSI based time-delay estimation block diagram using the righthand matrix from the second dimension of $\mathcal{S}^{\mathrm{c}}$

3.5 Simulation with $\left(L_{d}=2\right)$ signals for state-of-the-art 2nd Generation with $N=2046$ samples. Code samples are collected during $K=30$ epochs with $M=8$ antenna.

3.6 Simulation with $\left(L_{d}=3\right)$ signals for state-of-the-art 2 nd Generation with $N=2046$ samples. Code samples are collected during $K=30$ epochs with $M=8$ antenna. 
3.7 State-of-the-art CPD-GEVD, HOSVD SECSI, HOOI SECSI, and proposed SECSI method simulations using the first factor estimate with $M=8$ antennas and $L_{d}=2$ impinging signal. In both cases code samples are collected during $K=30$ epochs, and have $N=245520$ samples.

3.8 State-of-the-art CPD-GEVD, HOSVD SECSI, HOOI SECSI, and proposed SECSI method simulations using the second factor estimate with $M=8$ antennas and $L_{d}=2$ impinging signal. In both cases code samples are collected during $K=30$ epochs, and have $N=245520$ samples

3.9 State-of-the-art CPD-GEVD, HOSVD SECSI, HOOI SECSI, and proposed SECSI method simulations using the third factor estimate with $M=8$ antennas and $L_{d}=2$ impinging signal. In both cases code samples are collected during $K=30$ epochs, and have $N=245520$ samples.

3.10 State-of-the-art CPD-GEVD, HOSVD SECSI, HOOI SECSI, and proposed SECSI method simulations using the fourth factor estimate with $M=8$ antennas and $L_{d}=2$ impinging signal. In both cases code samples are collected during $K=30$ epochs, and have $N=245520$ samples.

3.11 State-of-the-art CPD-GEVD, HOSVD SECSI, HOOI SECSI, and proposed SECSI method simulations using the fifth factor estimate with $M=8$ antennas and $L_{d}=2$ impinging signal. In both cases code samples are collected during $K=30$ epochs, and have $N=245520$ samples.

3.12 State-of-the-art CPD-GEVD, HOSVD SECSI, HOOI SECSI, and proposed SECSI method simulations using the sixth factor estimate with $M=8$ antennas and $L_{d}=2$ impinging signal. In both cases code samples are collected during $K=30$ epochs, and have $N=245520$ samples.

3.13 State-of-the-art techniques and proposed SECSI method simulation with $M=8$ antennas and $L_{d}=2$ impinging signal. In both cases code samples are collected during $K=30$ epochs, and have $N=245520$ samples.

3.14 State-of-the-art techniques and proposed SECSI method simulation with $N=245520$ samples and $L_{d}=3$. In both cases code samples are collected during $K=30$ epochs with $M=8$ antenna.

3.15 State-of-the-art CPD-GEVD, HOSVD SECSI, HOOI SECSI, and proposed SECSI method simulations using the first factor estimate with $M=8$ antennas and $L_{d}=2$ impinging signal. In both cases code samples are collected during $K=30$ epochs, and have $N=245520$ samples.

3.16 State-of-the-art CPD-GEVD, HOSVD SECSI, HOOI SECSI, and proposed SECSI method simulations using the second factor estimate with $M=8$ antennas and $L_{d}=2$ impinging signal. In both cases code samples are collected during $K=30$ epochs, and have $N=245520$ samples 
3.17 State-of-the-art CPD-GEVD, HOSVD SECSI, HOOI SECSI, and proposed SECSI method simulations using the third factor estimate with $M=8$ antennas and $L_{d}=2$ impinging signal. In both cases code samples are collected during $K=30$ epochs, and have $N=245520$ samples.

3.18 State-of-the-art CPD-GEVD, HOSVD SECSI, HOOI SECSI, and proposed SECSI method simulations using the fourth factor estimate with $M=8$ antennas and $L_{d}=2$ impinging signal. In both cases code samples are collected during $K=30$ epochs, and have $N=245520$ samples

3.19 State-of-the-art CPD-GEVD, HOSVD SECSI, HOOI SECSI, and proposed SECSI method simulations using the fifth factor estimate with $M=8$ antennas and $L_{d}=2$ impinging signal. In both cases code samples are collected during $K=30$ epochs, and have $N=245520$ samples.

3.20 State-of-the-art CPD-GEVD, HOSVD SECSI, HOOI SECSI, and proposed SECSI method simulations using the sixth factor estimate with $M=8$ antennas and $L_{d}=2$ impinging signal. In both cases code samples are collected during $K=30$ epochs, and have $N=245520$ samples.

3.21 State-of-the-art techniques and proposed SECSI method simulation with $N=245520$ samples and $L_{d}=2$. In both cases code samples are collected during $K=30$ epochs with $M=8$ antenna....

3.22 State-of-the-art techniques and proposed SECSI method simulation with $N=245520$ samples and $L_{d}=3$. In both cases code samples are collected during $K=30$ epochs with $M=8$ antenna...

3.23 Proposed SECSI simulation with $L_{d}=2$ signals both 2 nd and 3rd with $N=$ 245520 samples. In both cases code samples are collected during $K=30$ epochs with $M=8$ antenna.

3.24 Proposed SECSI simulation with $L_{d}=3$ signals both 2 nd and 3rd with $N=$ 245520 samples. In both cases code samples are collected during $K=30$ epochs with $M=8$ antenna.

3.25 State-of-the-art techniques and Proposed SECSI simulation with $L_{d}=2 \mathrm{sig}$ nals for 2nd generation with $N=245520$ samples. In both cases code samples are collected during $K=30$ epochs with $M=8$ antenna.

3.26 State-of-the-art techniques and Proposed SECSI simulation with $L_{d}=2$ signals for 3rd Gen with $N=245520$ samples. In both cases code samples are collected during $K=30$ epochs with $M=8$ antenna.

3.27 State-of-the-art techniques and Proposed SECSI simulation with $L_{d}=3$ signals for 2 nd Gen with $N=245520$ samples. In both cases code samples are collected during $K=30$ epochs with $M=8$ antenna.

3.28 State-of-the-art techniques and Proposed SECSI simulation with $L_{d}=3 \mathrm{sig}$ nals for 3rd with $N=245520$ samples. In both cases code samples are collected during $K=30$ epochs with $M=8$ antenna. 
3.29 State-of-the-art techniques and Proposed SECSI simulation with $L_{d}=2$ signals for 2 nd Gen with $N=245520$ samples. In both cases code samples are collected during $K=30$ epochs with $M=8$ antenna.

3.30 State-of-the-art techniques and Proposed SECSI simulation with $L_{d}=3$ signals for 2 nd Gen with $N=245520$ samples. In both cases code samples are collected during $K=30$ epochs with $M=8$ antenna.

3.31 State-of-the-art techniques and Proposed SECSI simulation with $L_{d}=2$ signals for 2 nd Gen with $N=245520$ samples. In both cases code samples are collected during $K=30$ epochs with $M=8$ antenna.

3.32 State-of-the-art techniques and Proposed SECSI simulation with $L_{d}=3 \mathrm{sig}$ nals for 2 nd Gen with $N=245520$ samples. In both cases code samples are collected during $K=30$ epochs with $M=8$ antenna

A.1 Double-Arc positioner illustration

A.2 Double-Arc positioner final stage ............................................ 69

A.3 Double-Arc controller scheme ............................................. 70

A.4 Double-Arc web user interface ............................................... 71

B.1 To the left, perspective view of the brain of a mouse, with the shaded area corresponding to the somatosensory cortex. In the middle, cross section of this same brain. To the right, part of the somatosensory cortex is zoomed, showing a probe with several electrodes inserted for LFP recordings.

B.2 Experimental setting in which a mouse receives sensory stimulus every few seconds. To isolate the evoked potential in one electrode, one must average over several realizations of the stimulus. This procedure drastically increases the signal to noise ratio compared to a single realization.

B.3 Four CSD analyses: first CSD analysis of the original measurements, second CSD analysis of the first component with greatest power obtained with stICA, third CSD analysis of the second component with greatest power obtained with stICA, and fourth CSD analysis with the sum of the two components obtained with stICA

B.4 Four CSD analyses: first CSD analysis of the original measurements, second CSD analysis of the first component with greatest power, third CSD analysis of the second component with greatest power, and fourth CSD analysis with the sum of the two components obtained with real-valued PARAFAC approximation

B.5 Four CSD analyses using the proposed approach: first CSD analysis of the original measurements, second CSD analysis of the first component with greatest power, second CSD analysis of the second component with greatest power, and third CSD analysis with the sum of the two components obtained from the real-valued PARAFAC approximation with CWT.... 
B.6 Six CSD analyses using the proposed approach: first CSD analysis of the original measurements, second CSD analysis of the first component with greatest power, second CSD analysis of the second component with greatest power, third CSD analysis of the second component with greatest power, fourth CSD analysis of the second component with greatest power, fifth CSD analysis of the second component with greatest power, and sixth CSD analysis with the sum of the two components obtained from the real-valued PARAFAC approximation with CWT 


\section{Chapter 1}

\section{Introduction}

Global Navigation Satellite Systems (GNSS), such the American GPS, European Galileo, Russian GLONASS, and Chinese BeiDou, have been present in several critical applications such civilian aviation, autonomous driving [3], defense, and timing and synchronization of critical networks. Furthermore, GNSS can be used for automatic toll systems [2], and in precision farming for accurate application of fertilization and to enable constant usage of machinery 24 hours a day [4].

Aiming to compute the receiver position on the earth's surface, the GNSS receiver make use of line-of-sight (LOS) components from at least four satellites. Yet, as a consequence of the propagation environment, LOS reflections caused by, for instance, trees, poles, lamps, and buildings can occur generating multipath components, known as non-light-ofsight (NLOS) components. Thus, the NLOS components interferes on the LOS components. Once LOS signals can be corrupted by NLOS components, the time-delay Estimation and thus the receiver position can be severely deteriorated when using the state-of-the-art GNSS receivers [27, 28, 29].

In order to provide safety-critical applications (SCA) or liability critical applications (LCA), multi-antenna systems raised the focus of methods development for multipath mitigation for SCA and LCA applications [30, 31]. Currently there are few state-of-the-art tensorbased multipath mitigation methods applied to time delay-estimation. Firstly, [7] introduced a solution based on HOSVD low-rank approximation with Forward-Backward Averaging (FBA) [10, 11], and Expanded Spatial Smoothing (ESPS) [12, 13]. Then, [14] proposed a solution based on using Direction of Arrival (DoA) estimation and Khatri-Rao Factorization (KRF). Finally, [23] uses the Canonical-Polyadic Decomposition by Generalized Eigenvalue Decomposition (CPD-GEVD) for time-delay estimation.

In this thesis we propose to utilize the Semi-algebraic framework for approximate Canonical Polyadic Decomposition via Simultaneous Matrix Diagonalization (SECSI) [18, 19, 20]. The SECSI starts by applying the Higher Order Singular Value Decomposition (HOSVD) low-rank approximation [32] on the post-correlated received tensor signals. Then, several 
joint matrix diagonalization $[21,22]$ on the second dimension of the core tensor obtained from HOSVD in order to estimate complex amplitude related factor matrix. Next, the remaining factor matrices are estimated by using Least Squares Khatri-Rao Factorization (LSKRF) $[16,17]$. Thus, given the estimated factor matrices corresponding to the postcorrelated tensor signal, time-delay estimation can be computed for each LOS and NLOS components. In addition, we propose to use the SECSI based time-delay estimation with the second generation GPS system and the next third generation GPS system. Additionally, we explore the possibility to substitute the HOSVD low-rank approximation by the Higher Order Orthogonal Iteration (HOOI) low-rank approximation.

This thesis is structured as follows: Chapter 2 presents the pre- and post-correlation data model. Chapter 3 presents the tensor time-delay estimation approaches: in Section 3.1 both state-of-the-art HOSVD, DoA/KRF, and CPD-GEVD approaches are described; in Section 3.2, we present the proposed time-delay estimation method based on the SECSI decomposition; and Section 3.4 presents the results of Monte Carlo (MC) simulations. Chapter 4 draws the conclusions. Furthermore, in the Appendix section we introduce important concepts needed to understand this thesis as well as parallel works developed during master program course. Therefore, in Appendix A we introduce the notation used in this thesis, and review the matrix and tensor calculus used in the thesis. 


\section{Chapter 2}

\section{Data Model}

GNSS uses satellite constellations distributed in a known pattern, this pattern is maintained by a terrestrial segment that oversee each satellite's position, as well as issues corrections, and upload ephemeris data of each satellite needed to calculate the users' position on earth.

The GPS uses Code Division Multiple Access (CDMA) in order to give a unique identification to each satellite. In addition, CDMA spreads the signal over a wider bandwidth. This is known as spreading spectrum. Basically, the spreading occurs when the transmitted data sequence is multiplied by a higher bandwidth periodic chip code. This periodic chip code, known as pseudo-random sequence (PRS), increases the signal bandwidth while diminish its spectral power density. Thus, once CDMA gives unique PRS to each satellite, the spreading allows satellites to operate over the same frequency. This is possible because the spreading step spreads the signal over a wider bandwidth, making it more robust to interference. However, the spreading process decreases the signal to noise ratio (SNR).

Once the signal is spread over a spreading sequence, the recovery of each satellite's signal is performed by a de-spreading process. In order to de-spread the signal, we correlate the received signal by each known PRS. This process yield a processing gain of around $30 \mathrm{~dB}$, thus each satellite signal can be recovered.

In this chapter we firstly introduce the scenario considered in this thesis in Section 2.1. Then, in Section 2.2 we introduce the third generation GPS by presenting an overview of the process of generating the necessary codes for creating the new L1C pilot channel. Firstly, we describe how to generate the base sequence that is used to generate the unique codes of each satellite. Then, we explain how to generate the overlay and L1C pilot code, and we show how to generate the Time Multiplexed Binary Offset Carrier (TMBOC). Finally, in Sections 2.3 and 2.4 we describe how the signal tensor is constructed, and how the the post-correlation data model is constructed using the L1C pilot channel created after TMBOC modulation. 


\subsection{Data model scenario}

Similarly to [6], let us consider an antenna array based receiver with $M$ elements for a third generation GPS system. As shown in Figure 2.1, we assume that $D$ satellites have line of sight (LOS) with the GPS receiver and, for the $d$-th satellite, there are $L_{d}$ multipath components. Therefore, the total amount of multipath components $L$ is given by $L=\sum_{d=1}^{D} L_{d}$.

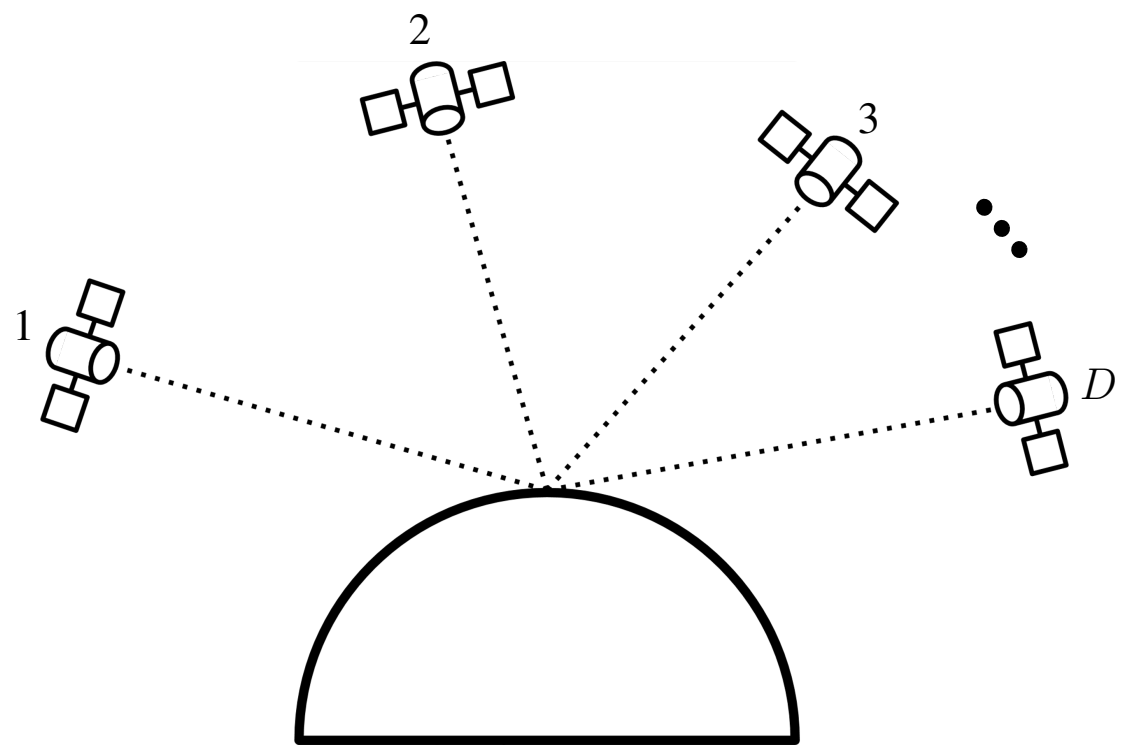

Figure 2.1: Satellite constellations

As illustrated in Figure 2.2, we assume that $\tau_{1}^{(d)}$ is the time delay of the LOS component of the $d$-satellite, while $\tau_{2}^{(d)}, \ldots, \tau_{L_{d}}^{(d)}$ are the time delays of the $\left(L_{d}-1\right)$ non-LOS (NLOS) components. Each satellite transmits $N$ symbols corresponding to the L1C pilot code modulated with TMBOC. We refer to the time window containing $N$ symbols as an epoch. Moreover, we assume that, during $K$ epochs, their respective $N$ symbols are approximately constant.

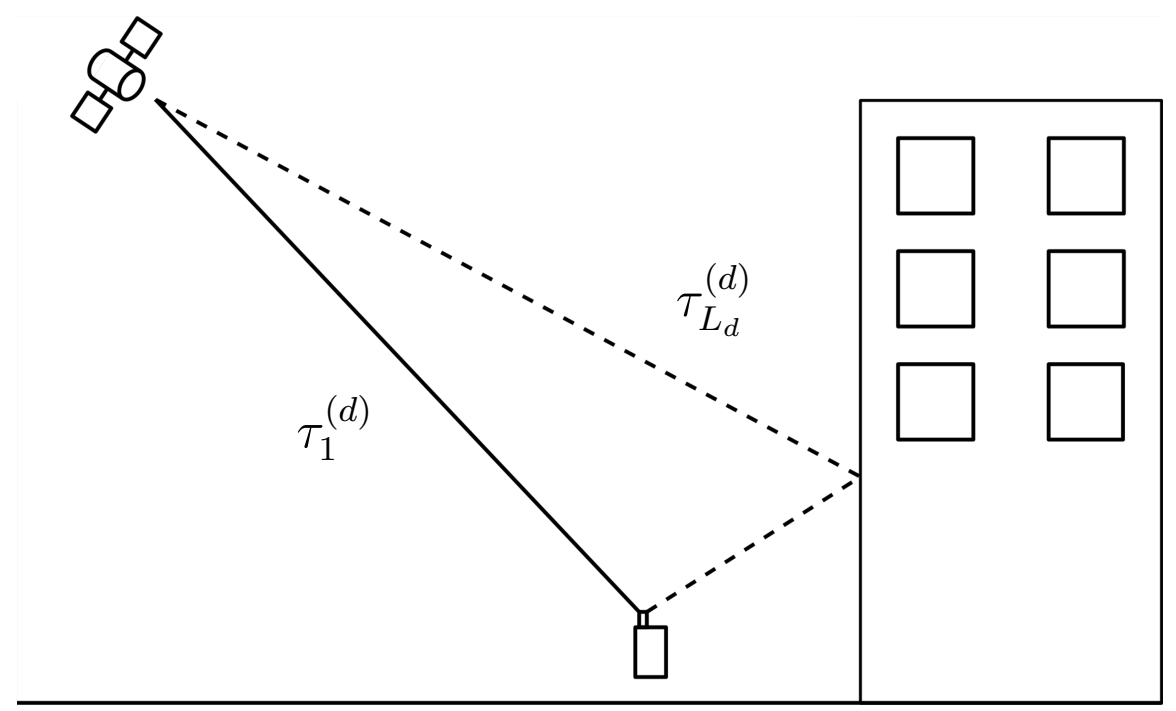

Figure 2.2: Receiver receiving one LOS e $L_{d}$ NLOS signals 


\subsection{L1C signal generation}

According to the specification IS-GPS-800D [33], each Pseudo-Random Sequence (PRS) of the L1C pilot code is composed of a unique spreading code and a unique overlay code called $L 1 C_{O}$. Both $\mathrm{L} 1 \mathrm{C}$ pilot and $L 1 C_{O}$ are independent and time synchronized. The L1C pilot code is generated with a chipping rate of $1.023 \mathrm{Mbps}$ and has a $10 \mathrm{~ms}$ duration, while the $L 1 C_{O}$ is generated with a $100 \mathrm{bps}$ rate, contains $1800 \mathrm{bits}$, and has $18 \mathrm{~s}$ duration. This $L 1 C_{O}$ is utilized to improve the correlation properties of the L1C pilot code, decrease spectral lines, and enables synchronization with the data message [34].

The L1C pilot code is generated by firstly creating a common 10223-bit Legendre Sequence $L_{\mathrm{S}}(t)$, for $t=0, \ldots, 10222$, assim construída

$$
\begin{aligned}
& L_{\mathrm{S}}(0)=0 \\
& L_{\mathrm{S}}(t)=1, \text { if there exists an integer } x \text { such that } t \text { is congruent to } x^{2} \text { modulo } 10223 \\
& L_{\mathbf{S}}(t)=0, \text { if there exists no integer } x \text { such that } t \text { is congruent to } x^{2} \text { modulo } 10223 .
\end{aligned}
$$

$L_{\mathrm{S}}(t)$ is then used to generate a Weil-Code $W_{i}(t ; w)$ by performing the exclusive-or (XOR) of $L_{\mathrm{S}}(t)$ and a shift of $L_{\mathrm{S}}(t)$. The shift of $L_{\mathrm{S}}(t)$ is specified by the Weil index $w$ ranging from 1 to 5111, and is defined as:

$$
W_{i}(t ; w)=L_{\mathrm{S}}(t) \oplus L_{\mathrm{S}}((t+w) \quad \bmod 10223),
$$

where $i$ is the PRN signal number, $\oplus$ denotes the XOR operation, mod is the modulo-2 operation, and $t=0 \ldots 10222$. Then, the L1C pilot codes are constructed by inserting a 7-bit fixed expansion sequence 0110100 into the Weil-code in the insertion point specified by the insertion index $p=1,2, \ldots, 10223$. This expansion sequence is inserted before the $p$ th value of the Weil-code, as illustrated in Figure 2.3.

Fixed Length-10223 Legendre Sequence

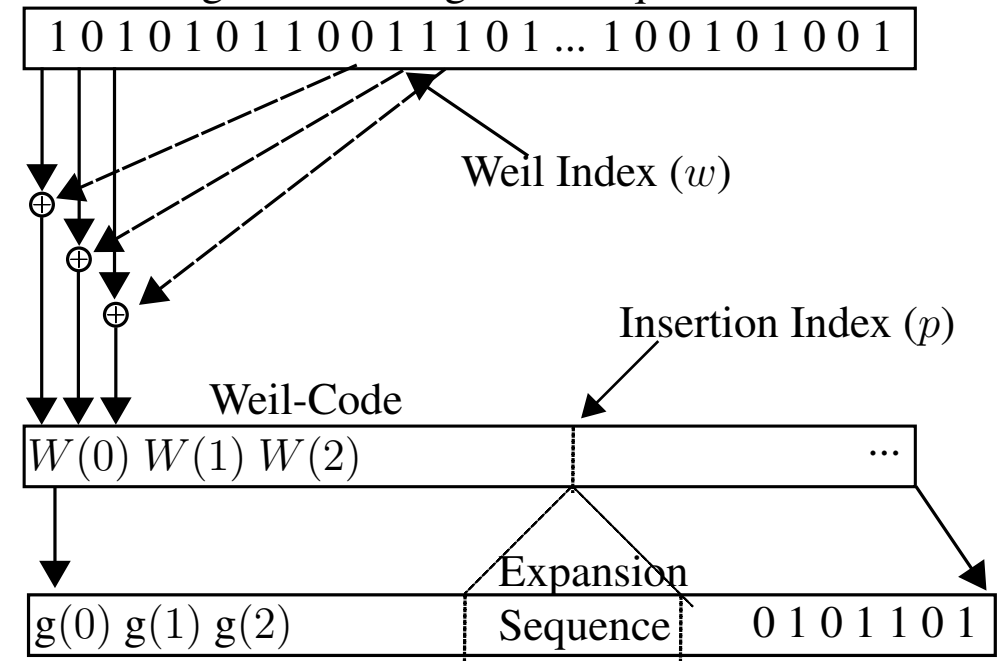

Figure 2.3: Generation of the L1C pilot code 
Next, the $L 1 C_{O}$ is constructed using an 11-stage Linear Feedback Shift Register (LFSR), as shown in Figure 2.4. The generated code, derived from a XOR of sequences S1 and S2, is 2047 bit long and truncated to a 1800 bit long sequence. Note that for sequence $\mathrm{S} 1$ each output of each tap is used to compute the feedback value while only taps 9 and 11 are used to calculate the feedback value of S2. Furthermore, from PRN 1 to 63 only sequence S1 is used whereas to all others PRN numbers the sequence S2 is introduced.

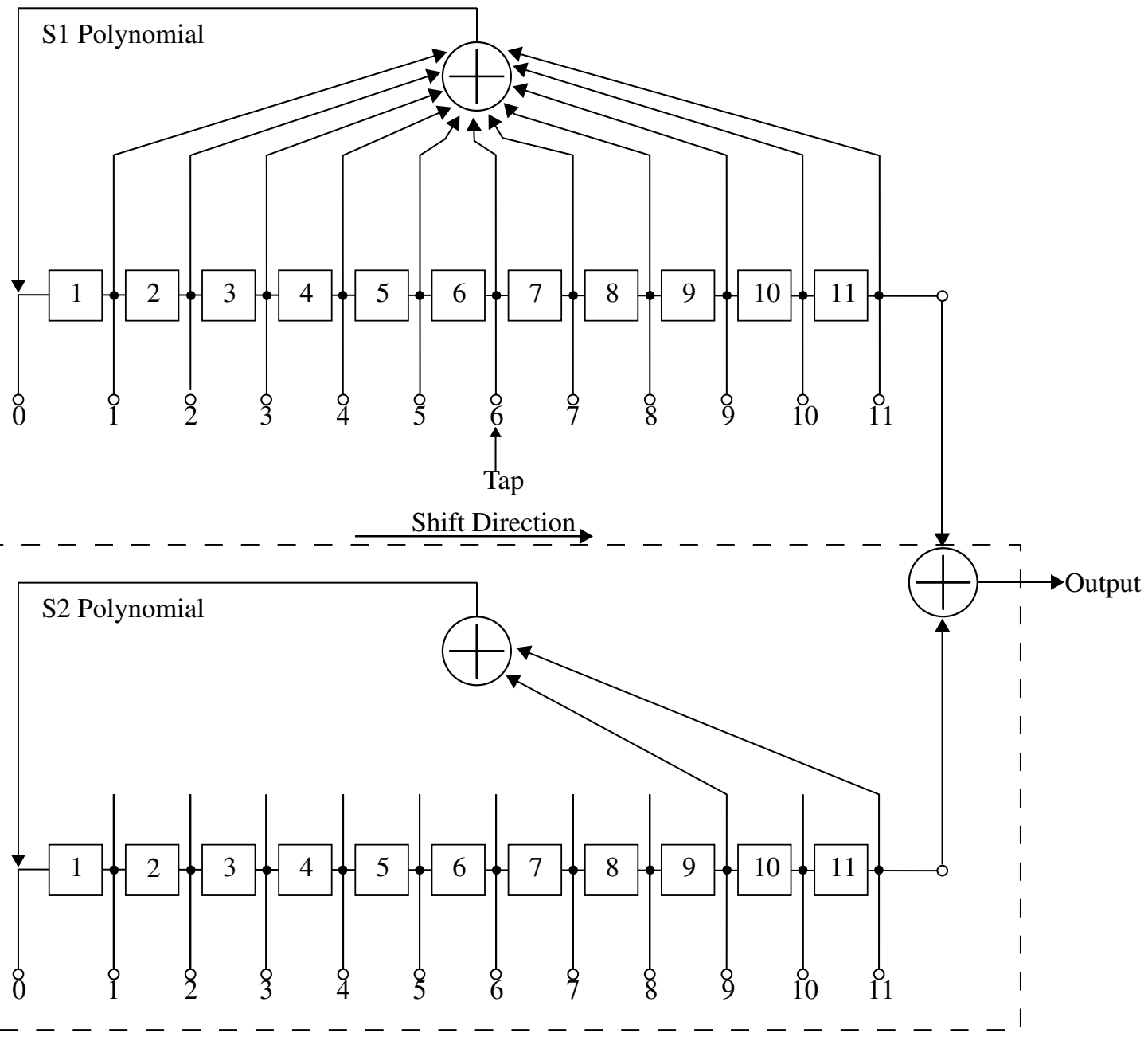

Figure 2.4: $L 1 C_{O}$ LFSR Generator

Then, the $L 1 C_{O}$ code is modulated into the $\mathrm{L} 1 \mathrm{C}$ pilot code. Since the $L 1 C_{O}$ code and the L1C pilot code have different duration, Figure 2.5 illustrates the time relationship between both codes. Observe that the GPS 12 week has a total duration of 18 seconds which corresponds to the time length of $L 1 C_{O}$ code. Therefore, each bit of $L 1 C_{O}$ is 10 milliseconds long which is equivalent to the duration of one period of L1C pilot code. 


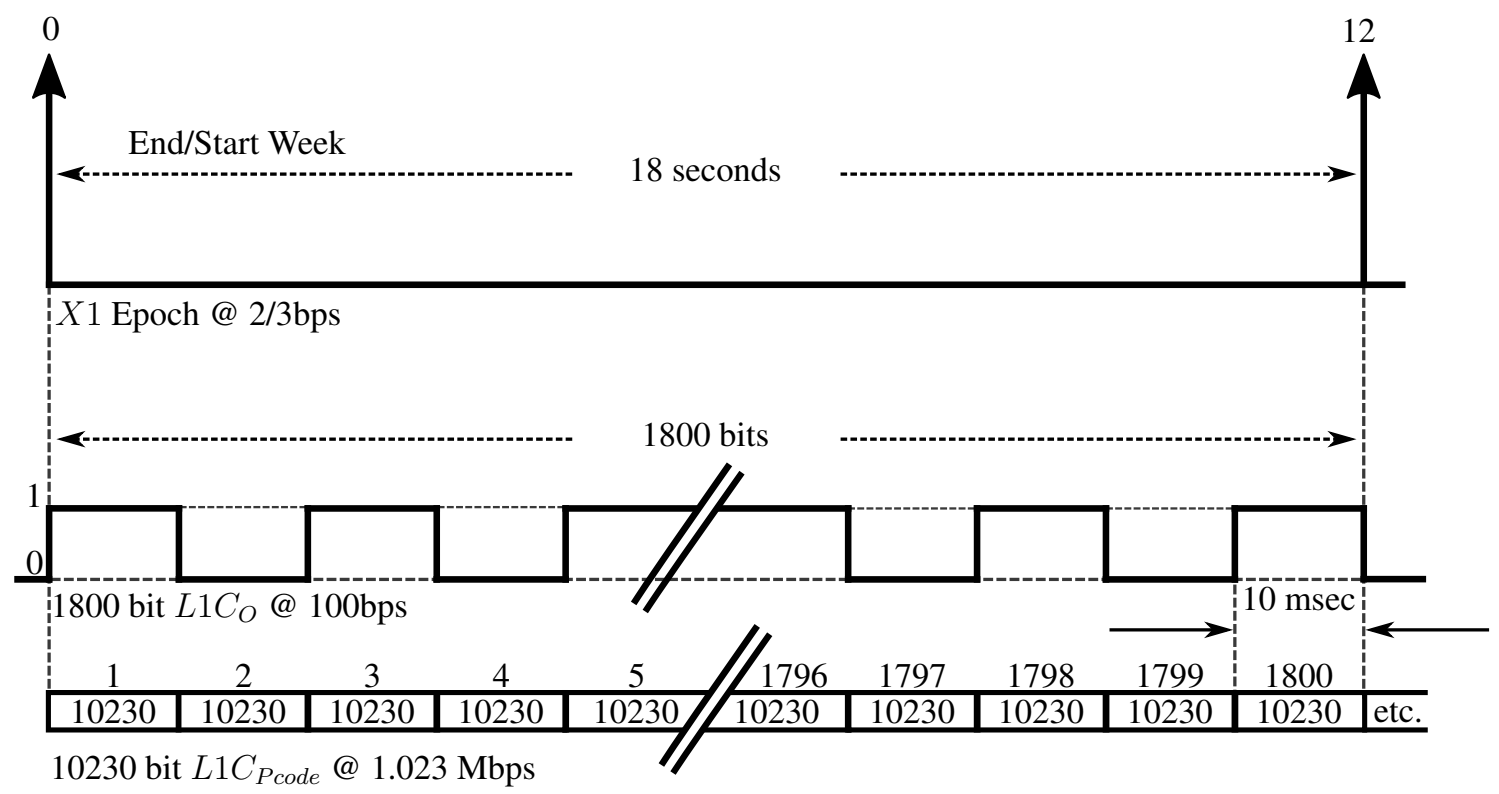

Figure 2.5: L1C pilot code and $L 1 C_{O}$ code time relationship

Finally, the L1C pilot code is modulated by a TMBOC subcarrier. The TMBOC is a mixture of $\operatorname{BOC}(1,1)$ subcarrier and $\operatorname{BOC}(6,1)$ subcarrier used to modulate the L1C pilot code into L1 carrier. $\mathrm{BOC}(1,1)$ has subcarrier frequency of $1.023 \mathrm{MHz}$ and a chipping rate of $1.023 \mathrm{Mbps}$ while $\mathrm{BOC}(6,1)$ consists of 6 cycles of a 6 x $1.023 \mathrm{MHz}$ squarewave, thus each squarewave is defined as:

$$
\mathbf{s}_{\mathrm{TMBOC}(6,1,4 / 33)}(t)= \begin{cases}\mathbf{s}_{\mathrm{BOC}(1,1)}(t) & t \in P_{1} \\ \mathbf{s}_{\mathrm{BOC}(6,1)}(t) & t \in P_{2},\end{cases}
$$

where $P_{1}$ is the set of chips where we apply $\operatorname{BOC}(1,1)$ and $P_{2}$ is the set of chips where we apply $\mathrm{BOC}(6,1), 4 / 33$ means 4 out of 33 chips of the spreading code are modulated by BOC $(6,1)$ subcarrier while the remaining 29 chips are modulated by $\mathrm{BOC}(1,1)$ subcarrier. Finally, in (2.4) the L1C pilot code is modulated using TMBOC, thus resulting in the L1C pilot channel.

$$
\tilde{\mathbf{c}}(t)=\mathbf{g}(t) \mathbf{s}_{\operatorname{TMBOC}(6,1,4 / 33)}(t),
$$

where $\mathrm{g}(t)$ is the L1C pilot code. Figure 2.6 illustrates the TMBOC modulation where each chip of the $\mathrm{L} 1 \mathrm{C}$ pilot code is multiplied by the resulting TMBOC squarewave thus resulting in the modulated L1C pilot channel. 

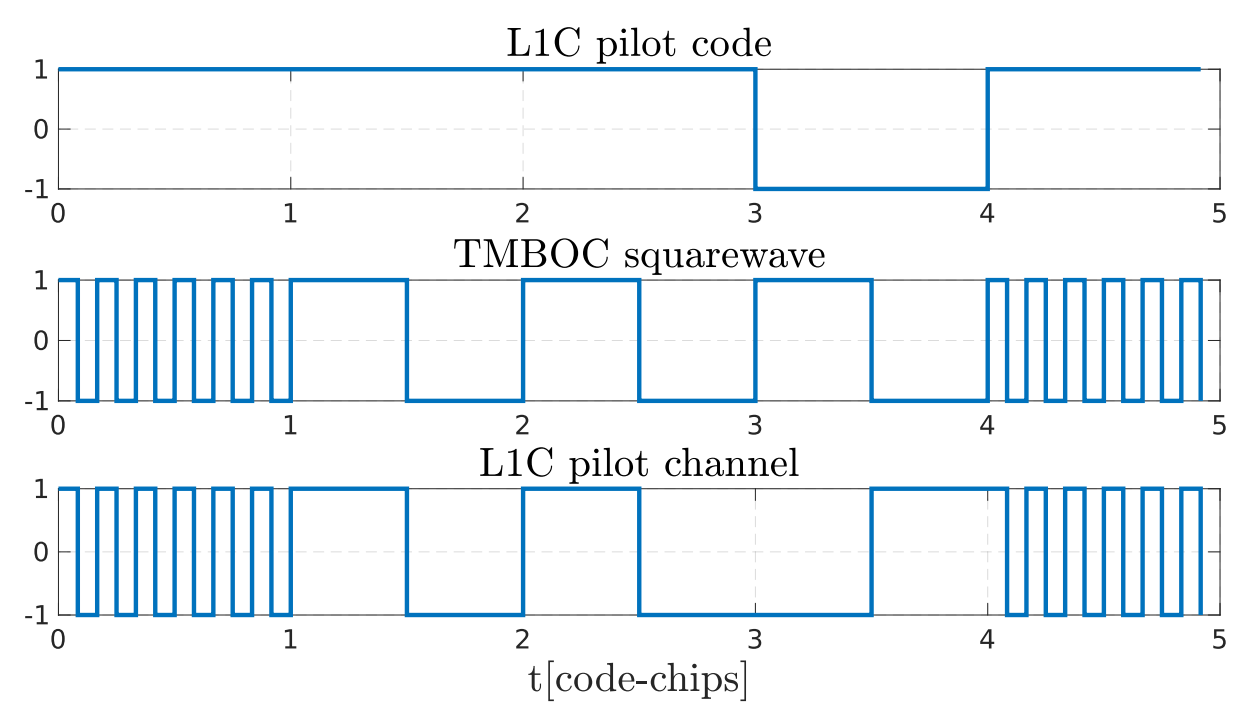

Figure 2.6: L1C pilot with TMBOC modulation

\subsection{Pre-Correlation Data Model}

Assuming a uniform linear antenna array (ULA) composed of $M$ elements and assuming $D$ satellites with $L$ impinging signals composed of 1 LOS and $L-1$ NLOS components, the received signal can be modeled in the following fashion

$$
\mathbf{s}_{d, \ell_{d}}(t)=\tilde{\mathbf{a}}\left(\phi_{d, \ell_{d}}\right) \tilde{\gamma}_{\ell_{d}} \tilde{\mathbf{c}}_{d}\left(t-\tau_{d, \ell_{d}}\right),
$$

where $\mathbf{s}_{d, \ell_{d}}(t)$ holds the desired LOS signal for $\ell_{d}=1$ along with the NLOS components for $\ell_{d}=2, \ldots, L_{d}$. The signal replica in (2.5) has its steering vector $\tilde{\mathbf{a}}\left(\phi_{d, \ell_{d}}\right)$, complex amplitude $\tilde{\gamma}_{\ell_{d}}$, and PRS $\tilde{\mathbf{c}}_{d}\left(t-\tau_{d, \ell_{d}}\right)$ with delay $\tau_{d, \ell_{d}}$, and the time index $t=1, \ldots, N$.

Considering that each antenna element captures $N$ samples along $K$ epochs the received signal tensor can be expressed in the following fashion:

$$
\mathcal{X}=\mathcal{I}_{3, L} \times{ }_{1} \tilde{\boldsymbol{\Gamma}}^{\mathrm{T}} \times{ }_{2} \tilde{\mathbf{C}}^{\mathrm{T}} \times{ }_{3} \tilde{\mathbf{A}}+\mathcal{N}
$$

where $\tilde{\Gamma}^{\mathrm{T}}=\left[\tilde{\gamma_{1}}, \ldots, \tilde{\gamma_{L}}\right] \in \mathbb{C}^{K \times L}$ gathers the complex amplitudes of the LOS and NLOS components in its columns, $\tilde{\mathbf{C}}=\left[\tilde{\mathbf{c}}\left[\tau_{1}\right], \ldots, \tilde{\mathbf{c}}\left[\tau_{L}\right]\right] \in \mathbb{R}^{N \times L}$ gathers the L1C pilot channel's PRS, $\tilde{\mathbf{A}}=\left[\tilde{\mathbf{a}}\left(\phi_{1}\right), \ldots, \tilde{\mathbf{a}}\left(\phi_{L}\right)\right] \in \mathbb{C}^{M \times L}$ collects the array responses, and $\mathcal{N}$ is a white Gaussian noise tensor.

The tensor in (2.6) is composed of three dimensions, the first dimensions of size $K$ is related to each epoch, the second dimensions of size $N$ is associated to the collected samples in each epoch, and the third dimension of size $M$ corresponds to the spatial diversity of the receive ULA. 


\subsection{Post-Correlation Data Model}

In order to separate each satellite, there are $D$ compressed correlator banks $\mathbf{Q}$ and each of them correspond to one satellite. Therefore, we define the $d$-th correlator bank as

$$
\mathbf{Q}=\left[\begin{array}{lll}
\mathbf{c}\left[\tau_{1}\right] & \cdots & \mathbf{c}\left[\tau_{Q}\right]
\end{array}\right] \in \mathbb{R}^{N \times Q} .
$$

Then, a Fisher information-preserving compression [8] is applied to the correlator bank by using the economy-size Singular Value Decomposition (SVD):

$$
\mathbf{Q}=\mathbf{U} \Sigma \mathbf{V}^{\mathrm{H}}
$$

with $\mathbf{U} \in \mathbb{C}^{N \times Q}, \boldsymbol{\Sigma} \in \mathbb{C}^{Q \times Q}$, and $\mathbf{V}^{\mathrm{H}} \in \mathbb{C}^{Q \times Q}$. Thus, since $\mathbf{U}$ preserves the statistics properties of the noise [35], we can define the compressed correlator bank as $\mathbf{Q}_{\omega}=\mathbf{U}$.

Thus, according to [7], a received signal tensor $\mathcal{X} \in \mathbb{C}^{K \times N \times M}$ can be correlated with the left subspace of the correlator bank $\mathbf{Q}_{\omega}=\mathrm{Q}\left(\Sigma \mathbf{V}^{\mathrm{H}}\right)^{-1}$ [8], where $\mathrm{Q}$ is a collection of $Q$ taps of the PRS, $\boldsymbol{\Sigma}$ and $\mathrm{V}^{\mathrm{H}}$ are, respectively, a diagonal matrix with the singular values and the right singular vector of the thin SVD of $\mathrm{Q}$. Thus, in order to compute the cross-correlation to estimate the time-delay of the LOS component, we have:

$$
\begin{aligned}
\mathcal{Y} & =\mathcal{X} \times{ }_{2} \mathrm{Q}_{\omega}{ }^{\mathrm{T}} \\
& =\mathcal{I}_{3, L} \times{ }_{1} \Gamma^{\mathrm{T}} \times_{2}\left(\mathrm{CQ}_{\omega}\right)^{\mathrm{T}} \times_{3} \mathrm{~A}+\mathcal{N} \times{ }_{2} \mathrm{Q}_{\omega} \\
& =\mathcal{I}_{3, L} \times{ }_{1} \Gamma^{\mathrm{T}} \times_{2}\left(\mathrm{CQ}_{\omega}\right)^{\mathrm{T}} \times_{3} \mathrm{~A}+\mathcal{N}_{\omega} .
\end{aligned}
$$

Thus, it results in the tensor $\mathcal{Y} \in \mathbb{C}^{K \times Q \times M}$, where $\Gamma^{\mathrm{T}} \in \mathbb{C}^{K \times L_{d}},\left(\mathbf{C Q}_{\omega}\right)^{\mathrm{T}} \in \mathbb{R}^{Q \times L_{d}}$, $\mathrm{A} \in \mathbb{C}^{M \times L_{d}}$, and $\mathcal{N}_{\omega}$ is a white Gaussian noise tensor. 


\section{Chapter 3}

\section{Tensor-Based Time-Delay Estimation and Simulations}

In this chapter we present three tensor-based time-delay solutions. Firstly the state-of-the-art tensor-based time-delay estimation HOSVD, DoA/KRF, and CPDGEVD from [7] and [23] are overviewed in Section 3.1. Still, in Subsection 3.1.2.1 we describe the time-delay estimation procedure by normalizing the estimated factors and estimating the tensor amplitudes. Then, a novel approach for time-delay estimation is presented in Section 3.2. This approach is based on [18, 19, 20] which uses a Semi-algebraic solution for the Canonical Polyadic (CP) model by performing several joint matrix diagonalizations. Thus, this solution simultaneously estimates the complex amplitude factor matrix. Then, we use the Least Square Khatri-Rao Factorization (LSKRF) to estimate the steering and code factor matrices. Section 3.3 presents the computational complexity of the state-of-the-art and proposed methods. Finally, In Section 3.4 we present the result for the Monte Carlo simulations performed using the state-of-the-art techniques and the proposed technique.

\subsection{State-Of-The-Art Tensor-Based Time-Delay Estima- tion For Third Generation GPS}

In this section we overview the state-of-the-art tensor-based time-delay estimation approaches. Firstly, in Subsection 3.1.1 we introduce an HOSVD based eigenfilter with Forward Backward Averaging (FBA) and Expanded Spatial Smoothing (ESPS). Then, in Subsection 3.1.2 we describe the Direction of Arrival (DoA) estimation and Khatri-Rao factorization approach. Finally, in Subsection 3.1.3 we present the Canonical Polyadic Decomposition by Generalized Eigenvalue Decomposition (CPD-GEVD). 


\subsubsection{HOSVD based Time-Delay Estimation}

As shown in Figure 3.1, in order to perform the Higher-Order Eigenfilter decomposition [7], first the incoming signal $\mathcal{Y}$ is pre-processed to incorporate the Forward-Backward Averaging (FBA) [10], [11] and Expanded Spatial Smoothing (ESPS) [12], [13].

Similarly to [10], the tensor-based FBA uses flipped identity matrices in order to duplicate the number of samples. Thus, the left-hand identity matrix $\Pi_{M} \in \mathbb{R}^{M \times M}$ is of size $M$ flipped along its vertical axis. Moreover, the right-hand identity matrix $\Pi_{K Q} \in \mathbb{R}^{K Q \times K Q}$ is of size $K Q$ flipped along its vertical axis. Then, the identity matrices are applied to the third-mode unfolding of the received signal tensor:

$$
\mathbf{Z}=\left[\begin{array}{ll}
{[\mathcal{Y}]_{3}} & \boldsymbol{\Pi}_{M}[\mathcal{Y}]_{3}^{*} \Pi_{K Q}
\end{array}\right] \in \mathbb{C}^{M \times 2 K Q}
$$

Then, similarly to [12], the tensor-based ESPS uses selection matrices that separate the antenna array into $L_{S}$ subarrays with $M_{S}=M-L_{S}+1$ elements. Therefore, we define the selection matrices as follows:

$$
\mathbf{J}_{\ell_{S}}=\left[\begin{array}{lll}
0_{M_{S} \times \ell_{S}-1} & \mathbf{I}_{M_{S}} & 0_{M_{S} \times L_{S}-1}
\end{array}\right] \in \mathbb{R}^{M_{S} \times M}
$$

where $\ell_{S}=1, \ldots, L_{S}$. Thus, we use the selection matrices to apply the spatial smoothing to the FBA unfolding of the received signal tensor from (3.1)

$$
\mathbf{W}=\left[\begin{array}{lll}
\mathbf{J}_{1} \mathbf{Z} & \cdots & \mathbf{J}_{L_{S}} \mathbf{Z}
\end{array}\right] \in \mathbb{C}^{M_{S} \times 2 L_{S} K Q}
$$

where $\mathbf{W}$ is folded back using the third-mode unfolding thus resulting in a forward-backward averaged spatially-smoothed fourth-order tensor $\mathcal{Z}_{E S P S} \in \mathbb{C}^{2 K \times Q \times M_{S} \times L_{S}}$.

Next, the resulting tensor $\mathcal{Z}_{E S P S}$ is used to perform the HOSVD rank-one approximation on the space and epoch dimensions.

$$
\mathcal{Z}_{E S P S}=\mathcal{R} \times{ }_{1} \mathbf{U}_{1} \times_{2} \mathbf{U}_{2} \times_{3} \mathbf{U}_{3} \times_{4} \mathbf{U}_{4}
$$

where $\mathcal{R} \in \mathbb{C}^{2 K \times Q \times M_{S} \times L_{S}}$ is the core tensor, $\mathbf{U}_{1} \in \mathbb{C}^{2 K \times 2 K}, \mathbf{U}_{2} \in \mathbb{C}^{Q \times Q}, \mathbf{U}_{3} \in \mathbb{C}^{M_{S} \times M_{S}}$, and $\mathbf{U}_{4} \in \mathbb{C}^{L_{S} \times L_{S}}$ are unitary matrices collecting singular vectors of each mode's unfolding [36] from (2.9).

Afterwards, once we assume the LOS component has the greatest power, the dominant singular vectors are multiplied by $\mathcal{Z}_{E S P S}$. Then, the resulting vector is multiplied by the $\Sigma \mathbf{V}^{\mathrm{H}}$ from the thin SVD of $\mathbf{Q}$. Thus, resulting in the $\mathbf{q}_{E S P S}$ vector.

$$
\mathbf{q}_{E S P S}=\left[\mathcal{Z}_{E S P S} \times{ }_{1}\left(\mathbf{u}_{1}^{(1)}\right)^{\mathrm{H}} \times_{3}\left(\mathbf{u}_{1}^{(3)}\right)^{\mathrm{H}} \times_{4}\left(\mathbf{u}_{1}^{(4)}\right)^{\mathrm{H}}\right] \boldsymbol{\Sigma} \mathbf{V}^{\mathrm{H}}
$$


where $\mathbf{q}_{E S P S}$ contains the multi-dimensionally filtered cross-correlation values at each tap of the correlator bank. The resulting vector in the correlator dimension is then interpolated using a cubic spline so that higher accuracy can be achieved.

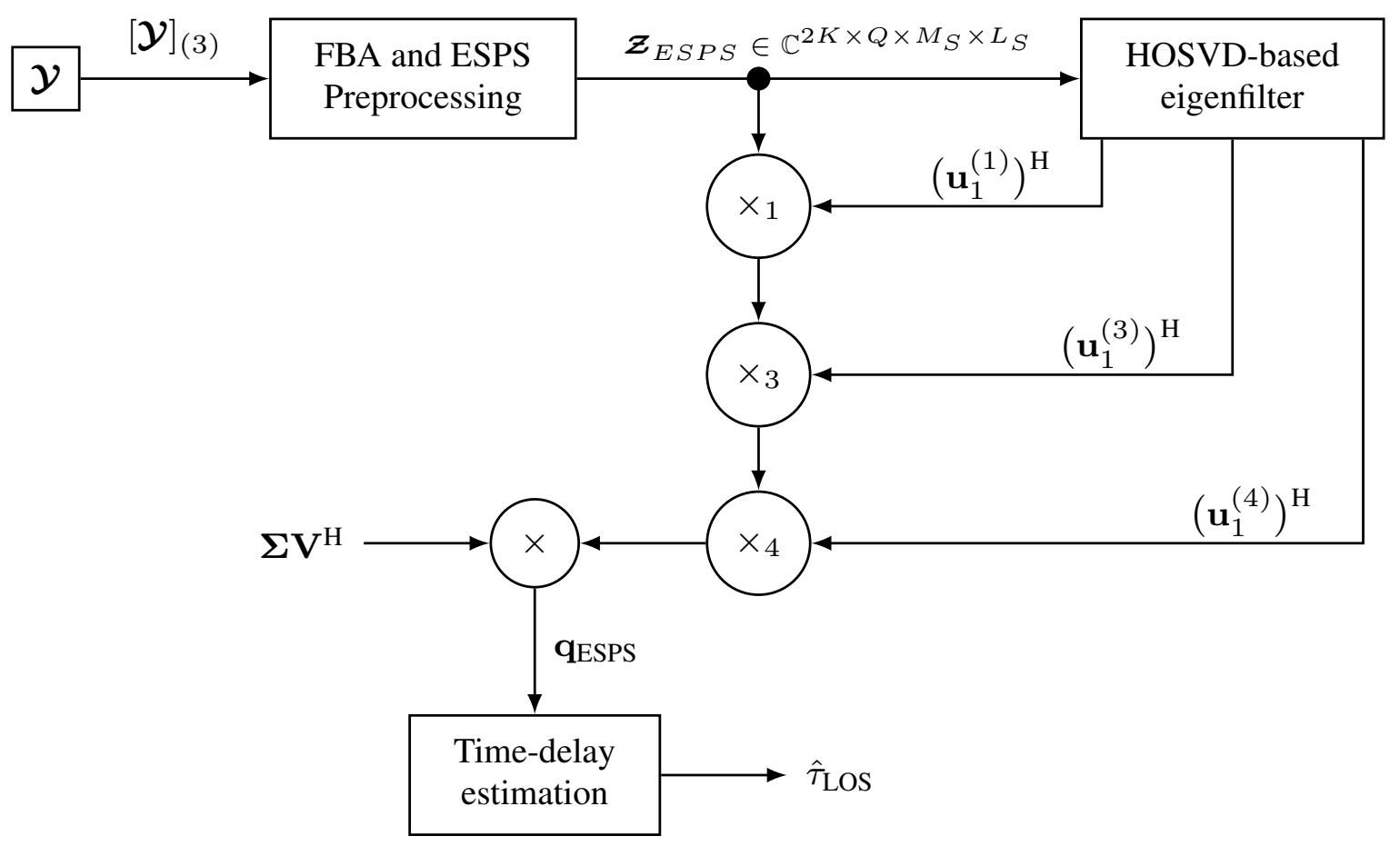

Figure 3.1: HOSVD Time-Delay Estimation block diagram

\subsubsection{DoA/KrF based Time-Delay Estimation}

A three step approach based on direction of arrival (DoA) estimation, the Khatri-Rao factorization (KRF), and the selection of the estimated LOS component was proposed by [14]. In order to perform DoA estimation, firstly the received signal tensor pre-processed using FBA and ESPS, thus we use the forward-backward averaged spatially smoothed signal matrix $\mathbf{W}$ from (3.3). As shown in Figure 3.2 the Estimation of Signal Parameter via Rotational Invariance Technique (ESPRIT) [15] is applied to $\mathbf{W}$ in order to estimate $\hat{\mathbf{A}}$.

Firstly we rewrite (2.9) to obtain the following equation

$$
[\boldsymbol{Y}]_{(3)}=\mathbf{A}\left[\Gamma^{\mathrm{T}} \diamond\left(\mathbf{C Q}_{\omega}\right)^{\mathrm{T}}\right]^{\mathrm{T}} \in \mathbb{C}^{M \times K Q}
$$

Once the matrix $\hat{\mathbf{A}}$ was estimated by the ESPRIT technique, its pseudo-inverse can be applied to (3.6) such that

$$
\begin{aligned}
\hat{\mathbf{A}}^{+}[\mathcal{Y}]_{3} & =\hat{\mathbf{A}}^{+} \mathbf{A}\left[\boldsymbol{\Gamma}^{\mathrm{T}} \diamond\left(\mathbf{C} \mathbf{Q}_{\omega}\right)^{\mathrm{T}}\right]^{\mathrm{T}} \\
& \approx\left[\boldsymbol{\Gamma}^{\mathrm{T}} \diamond\left(\mathbf{C Q}_{\omega}\right)^{\mathrm{T}}\right]^{\mathrm{T}} \in \mathbb{C}^{L_{d} \times K Q},
\end{aligned}
$$


where the factor matrices $\Gamma$ and $\left(\mathbf{C Q}_{\omega}\right)$ can be estimated by Least Square Khatri-Rao factorization (LSKRF) [16, 17].

Once $\left(\Gamma^{\mathrm{T}} \diamond\left(\mathbf{C Q}_{\omega}\right)^{\mathrm{T}}\right)^{\mathrm{T}}$ is given in (3.7), and considering that it's $\ell_{d}$-th column can be computed as the Khatri-Rao product of the $\ell_{d^{-}}$th column of $\Gamma^{\mathrm{T}}$ and $\left(\mathrm{CQ}_{\omega}\right)^{\mathrm{T}}$ :

$$
\left[\Gamma^{\mathrm{T}} \diamond\left(\mathbf{C Q}_{\omega}\right)^{\mathrm{T}}\right]\left(:, \ell_{d}\right)=\left(\Gamma^{\mathrm{T}}\right)\left(:, \ell_{d}\right) \diamond\left(\mathbf{C Q}_{\omega}\right)\left(:, \ell_{d}\right)
$$

where each column $\left(\boldsymbol{\Gamma}^{\mathrm{T}} \diamond\left(\mathbf{C Q}_{\omega}\right)^{\mathrm{T}}\right)\left(:, \ell_{d}\right) \in \mathbb{C}^{K Q}$. Thus, in order to solve the estimates of $\boldsymbol{\Gamma}$ and $\mathrm{CQ}_{\omega}$, we reshape (3.8) into a matrix of size $Q \times K$

$$
\underset{Q \times K}{\operatorname{unvec}}\left\{\left[\Gamma^{\mathrm{T}} \diamond\left(\mathbf{C Q}_{\omega}\right)^{\mathrm{T}}\right]\left(:, \ell_{d}\right)\right\}=\left(\mathbf{C Q}_{\omega}\right)^{\mathrm{T}}\left(:, \ell_{d}\right)\left(\boldsymbol{\Gamma}^{\mathrm{T}}\right)^{\mathrm{T}}\left(:, \ell_{d}\right)
$$

Once (3.9) is a rank-one matrix, we can perform the SVD-based rank-one approximation

$$
\underset{Q \times K}{\operatorname{unvec}}\left\{\left[\boldsymbol{\Gamma}^{\mathrm{T}} \diamond\left(\mathbf{C Q}_{\omega}\right)^{\mathrm{T}}\right]\left(:, \ell_{d}\right)\right\}=\mathbf{U}_{\ell_{d}} \boldsymbol{\Sigma}_{\ell_{d}} \mathbf{V}_{\ell_{d}}
$$

Therefore, the estimates for $\left(\boldsymbol{\Gamma}^{\mathrm{T}}\right)\left(:, \ell_{d}\right)$ and $\left(\left(\mathbf{C Q}_{\omega}\right)^{\mathrm{T}}\right)\left(:, \ell_{d}\right)$ are $\sqrt{\sigma_{\ell_{d}, 1}} \mathbf{v}_{\ell_{d}, 1}^{*}$ and $\sqrt{\sigma_{\ell_{d}, 1}} \mathbf{u}_{\ell_{d}, 1}$, respectively, where $\sigma_{\ell_{d}, 1}$ is the dominant singular value of $\Sigma_{\ell_{d}}, \mathbf{v}_{\ell_{d}, 1}^{*}$ is the conjugate of the dominant right singular vector of $\mathbf{V}_{\ell_{d}}$, and $\mathbf{u}_{\ell_{d}, 1}$ is the dominant left singular vector of $\mathbf{U}_{\ell_{d}}$. This is repeated for $\ell_{d}=1, \ldots, L_{d}$. 


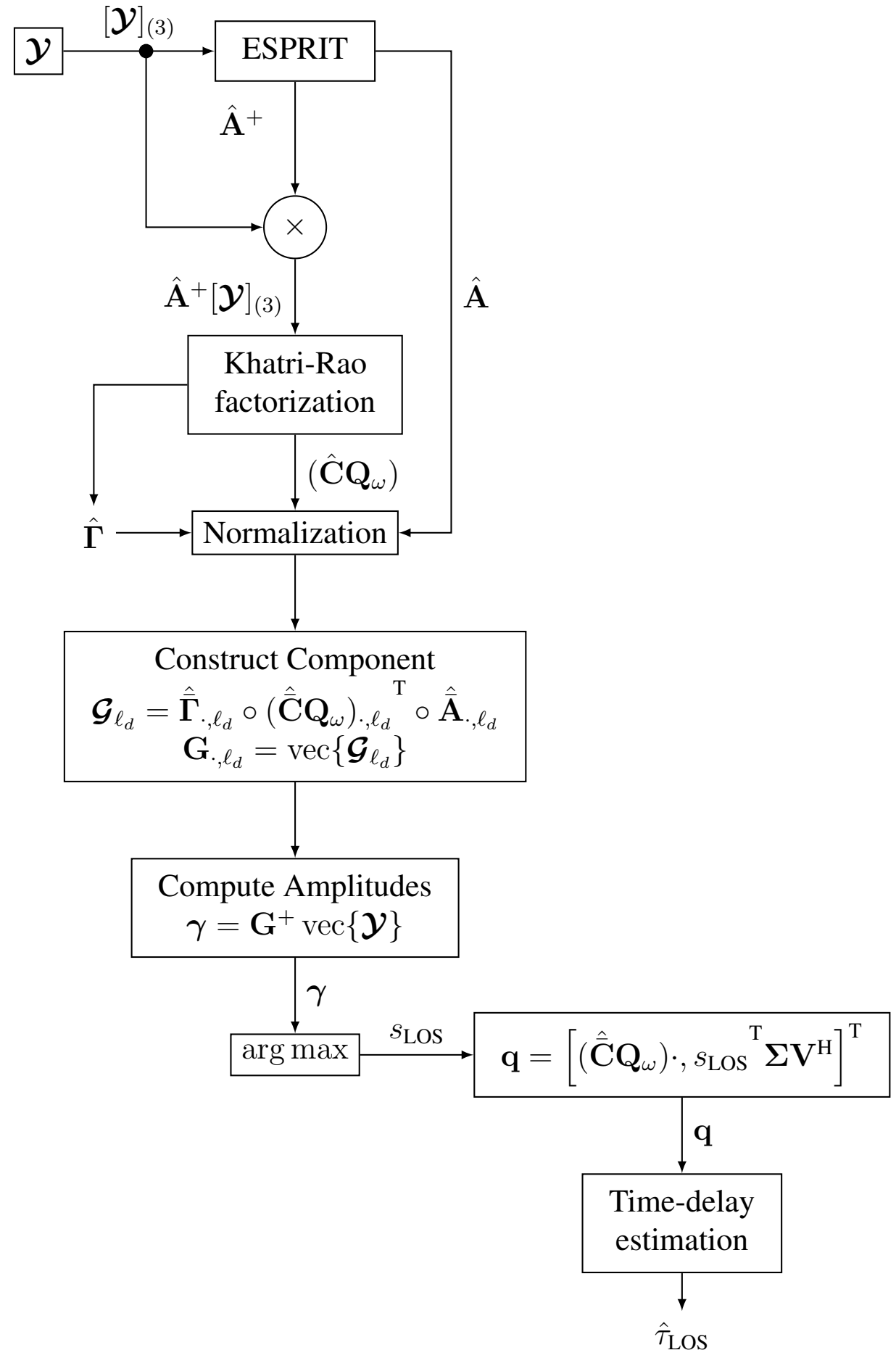

Figure 3.2: DoA/KrF Time-delay estimation block

\subsubsection{Time-Delay Estimation}

In this Subsection we describe the method used to perform the time-delay estimation after estimating the factor matrices. As shown in Figure 3.2, the resulting estimated factor matrices $\left(\hat{\mathbf{C}} \mathbf{Q}_{\omega}\right)^{\mathrm{T}}, \hat{\mathbf{A}}$, and $\hat{\boldsymbol{\Gamma}}$ are normalized to unit norm for the $\ell_{d^{-}}$th component 


$$
\begin{aligned}
\left(\hat{\overline{\mathbf{C}}} \mathbf{Q}_{\omega}\right)_{\cdot \ell_{d}}{ }^{\mathrm{T}} & =\left(\hat{\mathbf{C}} \mathbf{Q}_{\omega}\right)_{\cdot \ell_{d}}{ }^{\mathrm{T}} /\left\|\left(\hat{\mathbf{C}} \mathbf{Q}_{\omega}\right)_{\cdot, \ell_{d}}{ }^{\mathrm{T}}\right\|_{F} \\
\hat{\overline{\mathbf{A}}}_{\cdot \ell_{d}} & =\hat{\mathbf{A}}_{\cdot \ell_{d}} /\left\|\hat{\mathbf{A}}_{\cdot, \ell_{d}}\right\|_{F} \\
\hat{\overline{\boldsymbol{\Gamma}}}_{\cdot, \ell_{d}} & =\hat{\boldsymbol{\Gamma}}_{\cdot, \ell_{d}} /\left\|\hat{\boldsymbol{\Gamma}}_{\cdot, \ell_{d}}\right\|_{F} .
\end{aligned}
$$

Next, we construct the tensor $\mathcal{G}_{\ell_{d}}$ for the $\ell_{d}$-th normalized component of the estimated factor matrices from 3.13:

$$
\mathcal{G}_{\ell_{d}}=\hat{\overline{\boldsymbol{\Gamma}}}_{\cdot, \ell_{d}} \circ\left(\hat{\overline{\mathbf{C}}}_{\mathbf{Q}_{\omega}}\right)_{\cdot, \ell_{d}}^{\mathrm{T}} \circ \hat{\overline{\mathbf{A}}}_{\cdot, \ell_{d}}
$$

where $\mathcal{G}_{\ell_{d}} \in \mathbb{C}^{K \times Q \times M}$. Then, we store the tensor $\mathcal{G}_{\ell}$ corresponding to the $\ell_{d}$-th component in a matrix

$$
\mathbf{G}_{\cdot \ell_{d}}=\operatorname{vec}\left\{\mathcal{G}_{\ell_{d}}\right\}
$$

where $\mathbf{G} \in \mathbb{C}^{K Q M \times L_{d}}$. Thus, we can compute the tensor amplitudes by multiplying the pseudoinverse of $\mathrm{G}$ by the received signal tensor $\mathcal{Y}$ :

$$
\gamma=\mathrm{G}^{+} \operatorname{vec}\{\mathcal{Y}\}
$$

Then, in order to find the column of $\left(\hat{\overline{\mathbf{C}}} \mathbf{Q}_{\omega}\right)$ that corresponds to the line of sight component, we apply the arg max operator to the $\gamma$, and pick the $s_{\text {LOS }}$ column with greatest amplitude.

$$
s_{\mathrm{LOS}}=\arg \max \gamma
$$

Afterwards, we use the $s_{\text {LOS }}$ to select the LOS component from the estimated $\left(\hat{\overline{\mathbf{C}}} \mathbf{Q}_{\omega}\right)^{\mathrm{T}}$ and multiply it by $\Sigma \mathrm{V}^{\mathrm{H}}$ from the thin SVD of $\mathrm{Q}$

$$
\mathbf{q}=\left[\left(\hat{\overline{\mathbf{C}}} \mathbf{Q}_{\omega}\right)_{\cdot, s_{\mathrm{LOS}}}{ }^{\mathrm{T}} \boldsymbol{\Sigma} \mathbf{V}^{\mathrm{H}}\right]^{\mathrm{T}}
$$

Finally, the resulting vector $\mathbf{q} \in \mathbb{C}^{1 \times Q}$ is interpolated using a cubic spline in order to achieve a higher accuracy for the estimation of the time delay.

\subsubsection{CPD-GEVD based Time-Delay Estimation}

A more accurate tensor-based scheme has been proposed for time delay estimation was proposed in [9]. This method computes the Canonical Polyadic Decomposition, also known as Candecomp and PARAFAC, by Generalized Eigenvalue Decomposition. In Figure 3.3 we present a more accurate block diagram yet equivalent to the one presented in [23]. As illus- 
trated in Figure 3.3, the CPD-GEVD firstly computes the HOSVD low-rank approximation of the incoming signal $\mathcal{Y}$.

$$
\mathcal{Y} \approx \mathcal{S}^{\mathrm{g}} \times_{1} \mathrm{U}_{1}^{\mathrm{g}} \times_{1} \mathrm{U}_{2}^{\mathrm{g}} \times_{3} \mathrm{U}_{3}^{\mathrm{g}}
$$

where $\mathbf{U}_{1}^{\mathrm{g}} \in \mathbb{C}^{K \times L_{d}}, \mathbf{U}_{2}^{\mathrm{g}} \in \mathbb{C}^{Q \times L_{d}}$, and $\mathbf{U}_{3}^{\mathrm{g}} \in \mathbb{C}^{M \times L_{d}}$, and $\mathcal{S}^{\mathrm{g}} \in \mathbb{C}^{L_{d} \times L_{d} \times L_{d}}$ is the core tensor and can be expressed as a PARAFAC decomposition:

$$
\mathcal{S}^{\mathrm{g}}=\mathcal{I}_{3, L} \times_{1} \mathbf{T}_{1}^{\mathrm{g}} \times_{2} \mathbf{T}_{2}^{\mathrm{g}} \times_{3} \mathbf{T}_{3}^{\mathrm{g}},
$$

where the first two frontal slices of $\mathcal{S}^{\mathrm{g}}$ can be expressed as:

$$
\begin{aligned}
& \left(\mathcal{S}^{\mathrm{g}}\right)_{\cdot, \cdot, 1}=\mathbf{T}_{1}^{\mathrm{g}} \operatorname{diag}\left\{\left(\mathbf{T}_{3}^{\mathrm{g}}\right)_{\cdot, 1}\right\}\left(\mathbf{T}_{2}^{\mathrm{g}}\right)^{\mathrm{T}} \\
& \left(\mathcal{S}^{\mathrm{g}}\right)_{\cdot, \cdot, 2}=\mathbf{T}_{1}^{\mathrm{g}} \operatorname{diag}\left\{\left(\mathbf{T}_{3}^{\mathrm{g}}\right)_{\cdot, 2}\right\}\left(\mathbf{T}_{2}^{\mathrm{g}}\right)^{\mathrm{T}} .
\end{aligned}
$$

As shown in (3.22), the CPD-GEVD uses the eigenvectors, E, from the GEVD of the matrix pencil formed by $\left(\mathcal{S}^{\mathrm{g}}\right)_{,,, 1}$ and $\left(\mathcal{S}^{\mathrm{g}}\right)_{\cdot,, 2}$ :

$$
\left(\mathcal{S}^{\mathrm{g}}\right)_{,,, 1}^{\mathrm{T}} \mathbf{E}=\left(\mathcal{S}^{\mathrm{g}}\right)_{{ }_{r,, 2}^{\mathrm{T}}}^{\mathrm{T}} \mathbf{E D}
$$

where $\mathbf{D}$ the eigenvalues in its diagonal. Note that (3.22) is equivalent to

$$
\begin{aligned}
\left(\mathcal{S}^{\mathrm{g}}\right)_{\cdot, \cdot, 2}^{-\mathrm{T}}\left(\mathcal{S}^{\mathrm{g}}\right)_{\cdot, \cdot, 1}^{\mathrm{T}} & =\mathbf{E D E}^{-1} \\
& =\left(\mathbf{T}_{1}^{\mathrm{g}}\right)^{-\mathrm{T}} \operatorname{diag}\left\{\left(\mathbf{T}_{3}^{\mathrm{g}}\right)_{\cdot, 2}\right\}^{-1} \operatorname{diag}\left\{\left(\mathbf{T}_{3}^{\mathrm{g}}\right)_{\cdot, 1}\right\} \mathbf{T}_{1}^{\mathrm{g}} .
\end{aligned}
$$

Equation (3.23) is a diagonalization problem in which $\mathbf{E}$ provides an estimate of $\left(\mathbf{T}_{1}^{\mathrm{g}}\right)^{-\mathrm{T}}$. Therefore, by combining $\left(\mathbf{T}_{1}^{\mathrm{g}}\right)^{-\mathrm{T}}$ from (3.23) and $\mathbf{U}_{1}^{*}$ from (3.19) the factor matrix $\boldsymbol{\Gamma}^{-\mathrm{T}}$ can be computed as

$$
\hat{\boldsymbol{\Gamma}}^{+\mathrm{T}}=\left(\mathbf{U}_{1}^{\mathrm{g}}\right)^{*}\left(\mathbf{T}_{1}^{\mathrm{g}}\right)^{-\mathrm{T}}=\left(\mathbf{U}_{1}^{\mathrm{g}}\right)^{*} \mathbf{E} .
$$

Next, since in the noiseless case, according to (2.9),

$$
[\mathcal{Y}]_{(1)}^{\mathrm{T}}=\left[\left(\mathbf{C Q}_{\omega}\right) \diamond \mathbf{A}\right] \Gamma^{\mathrm{T}}
$$


and since $\hat{\boldsymbol{\Gamma}}^{-\mathrm{T}}$ is given in (3.24), we define $\mathbf{F}^{\mathrm{g}(2,3)}$ as

$$
\begin{aligned}
\mathbf{F}^{\mathrm{g}(2,3)} & =[\mathcal{Y}]_{(1)}^{\mathrm{T}}\left(\mathbf{U}_{1}^{\mathrm{g}}\right)^{*} \mathbf{E}=\left[\left(\mathbf{C Q}_{\omega}\right)^{\mathrm{T}} \diamond \mathbf{A}\right] \boldsymbol{\Gamma} \hat{\boldsymbol{\Gamma}}^{+} \\
& \approx\left[\left(\mathbf{C Q}_{\omega}\right)^{\mathrm{T}} \diamond \mathbf{A}\right] \in \mathbb{C}^{Q M \times L} .
\end{aligned}
$$

Then by performing the Least Squares Khatri-Rao Factorization (LSKRF)[16, 17], we can estimate the factor matrices $\left(\hat{\mathbf{C}} \mathbf{Q}_{\omega}\right)^{\mathrm{T}}$ and $\hat{\mathbf{A}}$. Furthermore, we use the estimated factor matrices to perform the time-delay estimation technique described in Subsection ?? 


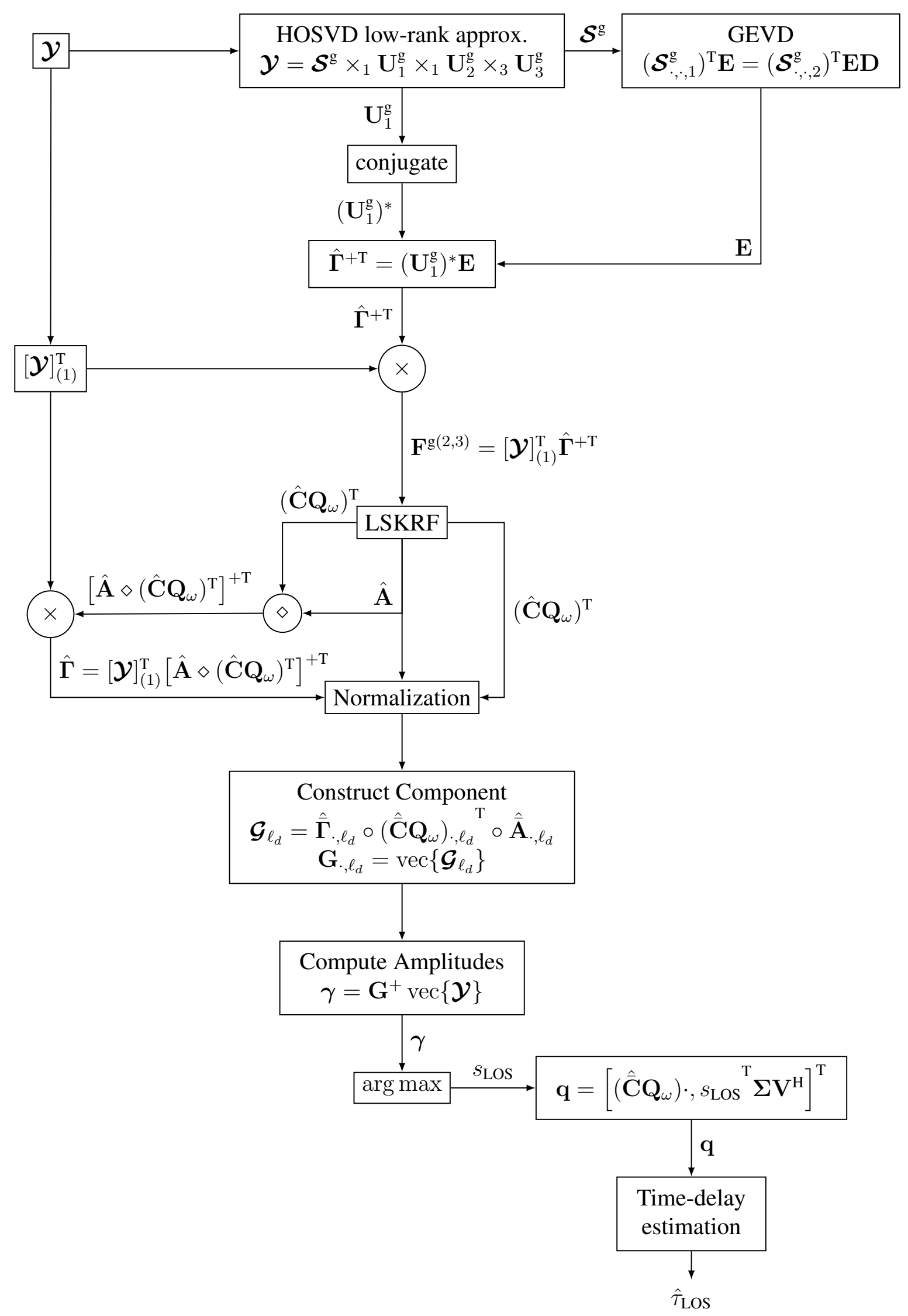

Figure 3.3: CPD-GEVD Time-Delay Estimation block diagram 


\subsection{Proposed Semi-algebraic Framework for Approximate Canonical Polyadic Decomposition via Simultaneous Matrix Diagonalization Based Framework for Time- Delay Estimation}

In this section, we propose to utilize the Semi-algebraic framework for approximate Canonical Polyadic Decomposition via simultaneous matrix diagonalization (SECSI) from $[18,19,20]$ to transforming our factor estimation problem into several redundant simultaneous matrix diagonalization problems. Therefore, we propose a SECSI based framework for time-delay estimation. Thus, firstly, as illustrated in Figure 3.4, the SECSI approach computes the Higher Order Singular Value Decomposition (HOSVD) low-rank approximation, which computes the thin-SVD of the incoming signal $\mathcal{Y}$ from Equation (2.9) is:

$$
\mathcal{Y}=\mathcal{S}^{\mathrm{c}} \times{ }_{1} \mathrm{U}_{1}^{\mathrm{c}} \times{ }_{2} \mathrm{U}_{2}^{\mathrm{c}} \times{ }_{3} \mathrm{U}_{3}^{\mathrm{c}}
$$

where $\mathcal{S}^{\mathrm{c}} \in \mathbb{C}^{L_{d} \times L_{d} \times L_{d}}$ is the compressed core tensor, and $\mathbf{U}_{1}^{\mathrm{c}} \in \mathbb{C}^{K \times L_{d}}, \mathbf{U}_{2}^{\mathrm{c}} \in \mathbb{C}^{Q \times L_{d}}$, $\mathbf{U}_{3}^{\mathrm{c}} \in \mathbb{C}^{M \times L_{d}}$ are the singular matrices. Thus, we can represent the tensor $\mathcal{S}$ as follows

$$
\mathcal{S}^{\mathrm{c}}=\mathcal{I} \times{ }_{1} \mathbf{T}_{1}^{\mathrm{c}} \times{ }_{2} \mathbf{T}_{2}^{\mathrm{c}} \times{ }_{3} \mathbf{T}_{3}^{\mathrm{c}},
$$

where $\mathbf{T}_{1}^{\mathrm{c}} \in \mathbb{C}^{L_{d} \times L_{d}}, \mathbf{T}_{2}^{\mathrm{c}} \in \mathbb{C}^{L_{d} \times L_{d}}$, and $\mathbf{T}_{3}^{\mathrm{c}} \in \mathbb{C}^{L_{d} \times L_{d}}$. Thus

$$
\begin{array}{r}
\mathbf{U}_{1}^{\mathrm{c}} \mathbf{T}_{1}^{\mathrm{c}}=\boldsymbol{\Gamma}^{\mathrm{T}}, \\
\mathbf{U}_{2}^{\mathrm{c}} \mathbf{T}_{2}^{\mathrm{c}}=\left(\mathbf{C Q}_{\omega}\right)^{\mathrm{T}}, \\
\mathbf{U}_{3}^{\mathrm{c}} \mathbf{T}_{3}^{\mathrm{c}}=\mathbf{A}^{\mathrm{T}},
\end{array}
$$

Once we perform joint matrix diagonalization on our tensor $\mathcal{S}^{\mathrm{c}}$, we have several diagonalization problems to be solved. Because we have a third-order tensor, we can have six different estimates which means we have two estimates for each dimension of tensor $\mathcal{S}^{\mathrm{c}}$. Thus, in order to compute the joint matrix diagonalization we firstly compute the first-, second-, and third-mode slice of the $i$-th slice of tensor $\mathcal{S}^{\mathrm{c}}$. Thus, we have the third-mode slice of $\mathcal{S}^{\mathrm{c}}$ represented as

$$
\begin{aligned}
\mathbf{S}_{3, i}^{c} & =\left[\left(\mathcal{S}^{\mathrm{c}} \times{ }_{3} \mathbf{U}_{3}^{\mathrm{c}}\right) \times{ }_{3} \mathbf{e}_{i}^{\mathrm{T}}\right] \\
& =\mathbf{T}_{1}^{\mathrm{c}} \operatorname{diag}\left\{\mathbf{A}^{\mathrm{H}}(:, i)\right\}\left(\mathbf{T}_{2}^{\mathrm{c}}\right)^{\mathrm{T}},
\end{aligned}
$$


second-mode slice of $\mathcal{S}^{\mathrm{c}}$ as

$$
\begin{aligned}
\mathbf{S}_{2, i}^{c} & =\left[\left(\mathcal{S}^{\mathrm{c}} \times{ }_{2} \mathbf{U}_{2}^{\mathrm{c}}\right) \times_{2} \mathbf{e}_{i}^{\mathrm{T}}\right] \\
& =\mathbf{T}_{1}^{\mathrm{c}} \operatorname{diag}\left\{\left(\mathbf{C} \mathbf{Q}_{\omega}\right)^{\mathrm{H}}(:, i)\right\}\left(\mathbf{T}_{3}^{\mathrm{c}}\right)^{\mathrm{T}},
\end{aligned}
$$

and, finally, we have the first-mode slice

$$
\begin{aligned}
\mathbf{S}_{1, i}^{c} & =\left[\left(\mathcal{S}^{\mathrm{c}} \times{ }_{1} \mathbf{U}_{1}^{\mathrm{c}}\right) \times{ }_{1} \mathbf{e}_{i}^{\mathrm{T}}\right] \\
& =\mathbf{T}_{2}^{\mathrm{c}} \operatorname{diag}\left\{\boldsymbol{\Gamma}^{\mathrm{H}}(:, i)\right\}\left(\mathbf{T}_{3}^{\mathrm{c}}\right)^{\mathrm{T}},
\end{aligned}
$$

then

$$
\begin{aligned}
& \mathbf{S}_{3, p}^{c}=\mathbf{T}_{1}^{\mathrm{c}} \operatorname{diag}\left\{\mathbf{A}^{\mathrm{H}}(:, p)\right\}\left(\mathbf{T}_{2}^{\mathrm{c}}\right)^{\mathrm{T}}, \\
& \mathbf{S}_{2, p}^{c}=\mathbf{T}_{1}^{\mathrm{c}} \operatorname{diag}\left\{\left(\mathbf{C Q}_{\omega}\right)^{\mathrm{H}}(:, p)\right\}\left(\mathbf{T}_{3}^{\mathrm{c}}\right)^{\mathrm{T}}, \\
& \mathbf{S}_{1, p}^{c}=\mathbf{T}_{2}^{\mathrm{c}} \operatorname{diag}\left\{\boldsymbol{\Gamma}^{\mathrm{H}}(:, p)\right\}\left(\mathbf{T}_{3}^{\mathrm{c}}\right)^{\mathrm{T}},
\end{aligned}
$$

where $\mathbf{e}_{i}^{\mathrm{T}}$ is vector with zeros in all positions except in the $i$-th position, and $p$ is an arbitrary index between one and the $n$-th mode slice to be diagonalized:

$$
p=\arg \min _{i} \operatorname{cond}\left\{\mathbf{S}_{n, i}^{\mathrm{c}}\right\}
$$

where $\operatorname{cond}\{\cdot\}$ computes the condition number of a matrix. The smaller the condition number, the more stable is the matrix inversion. Therefore, we select the matrix with the smallest condition number.

Therefore, with each mode slice defined, we can compute the right-hand and left-hand matrices of each mode slice. Note that the right-hand matrix simultaneous diagonalization for the third-mode slice is similar to the state-of-the-art CPD-GEVD described in Subsection 3.1.3. Since $p$ is fixed, we can vary all possible values of $i$, thus obtaining $N-1$ equations for each mode slice, since $i \neq p$.

Thus, firstly, we define the right-hand matrix for the third-mode slice in the following fashion:

$$
\begin{aligned}
\mathbf{S}_{3, i}^{\mathrm{c}, \mathrm{rhs}} & =\mathbf{S}_{3, i}^{\mathrm{c}}\left(\mathbf{S}_{3, p}^{\mathrm{c}}\right)^{-1} \\
& =\mathbf{T}_{1}^{\mathrm{c}} \operatorname{diag}\left\{\mathbf{A}^{\mathrm{H}}(:, i) \mathbf{A}^{\mathrm{H}}(:, p)\right\}\left(\mathbf{T}_{1}^{\mathrm{c}}\right)^{-1} \\
& =\mathbf{T}_{1}^{\mathrm{c}} \mathbf{A}^{\mathrm{H}}\left(\mathbf{T}_{1}^{\mathrm{c}}\right)^{-1}
\end{aligned}
$$


then, we define the left-hand matrix for the third-mode slice as follows:

$$
\begin{aligned}
\mathbf{S}_{3, i}^{\mathrm{c}, \text { lhs }} & =\left(\left(\mathbf{S}_{3, p}^{\mathrm{c}}\right)^{-1} \mathbf{S}_{3, i}^{\mathrm{c}}\right)^{\mathrm{T}}=\left(\mathbf{S}_{3, i}^{\mathrm{c}}\right)^{\mathrm{T}}\left(\mathbf{S}_{3, p}^{\mathrm{c}}\right)^{-T} \\
& =\mathbf{T}_{1}^{\mathrm{c}} \operatorname{diag}\left\{\mathbf{A}^{\mathrm{H}}(:, i) \mathbf{A}^{\mathrm{H}}(:, p)\right\}\left(\mathbf{T}_{2}^{\mathrm{c}}\right)^{-1} \\
& =\mathbf{T}_{2}^{\mathrm{c}} \mathbf{A}^{\mathrm{H}}\left(\mathbf{T}_{2}^{\mathrm{c}}\right)^{-1} .
\end{aligned}
$$

This way, we transformed the third-mode slice onto two joint matrix diagonalization problems. Thus, we can obtain an estimate of $\mathbf{A}$ from the joint matrix diagonalization of $\mathbf{S}_{3, i}^{\mathrm{c}, \text { rhs }}$. Additionally, we can obtain an estimate of $\mathbf{A}$ from the joint diagonalization of $\mathbf{S}_{3, i}^{\mathrm{c}, \mathrm{lhs}}$. Furthermore, the matrices that diagonalize $\mathbf{S}_{3, i}^{\mathrm{c}, \mathrm{rhs}}$ and $\mathbf{S}_{3, i}^{\mathrm{c}, \mathrm{lhs}}$ outputs estimates for $\mathbf{T}_{1}^{\mathrm{c}}$, and $\mathbf{T}_{2}^{\mathrm{c}}$. Thus, we can obtain the estimates for $\mathbf{U}_{1}^{\mathrm{c}} \mathbf{T}_{1}^{\mathrm{c}}=\hat{\boldsymbol{\Gamma}}^{\mathrm{T}}$, and $\mathbf{U}_{2}^{\mathrm{c}} \mathbf{T}_{2}^{\mathrm{c}}=\left(\hat{\mathbf{C}} \mathbf{Q}_{\omega}\right)^{\mathrm{T}}$.

Similarly, for the second-mode slice, we compute the right-hand matrix in the following fashion:

$$
\begin{aligned}
\mathbf{S}_{2, i}^{\mathrm{c}, \text { rhs }} & =\mathbf{S}_{2, i}^{\mathrm{c}}\left(\mathbf{S}_{2, p}^{\mathrm{c}}\right)^{-1} \\
& =\mathbf{T}_{1}^{\mathrm{c}} \operatorname{diag}\left\{\left(\mathbf{C Q}_{\omega}\right)^{\mathrm{H}}(:, i)\left(\mathbf{C Q}_{\omega}\right)^{\mathrm{H}}(:, p)\right\}\left(\mathbf{T}_{1}^{\mathrm{c}}\right)^{-1} \\
& =\mathbf{T}_{1}^{\mathrm{c}}\left(\mathbf{C Q}_{\omega}\right)^{\mathrm{H}}\left(\mathbf{T}_{1}^{\mathrm{c}}\right)^{-1}
\end{aligned}
$$

then we define left-hand matrix of the second-mode slice

$$
\begin{aligned}
\mathbf{S}_{2, i}^{\mathrm{c}, \text { lhs }} & =\left(\left(\mathbf{S}_{2, p}^{\mathrm{c}}\right)^{-1} \mathbf{S}_{2, i}^{\mathrm{c}}\right)^{T}=\left(\mathbf{S}_{2, i}^{\mathrm{c}}\right)^{\mathrm{T}}\left(\mathbf{S}_{2, p}^{\mathrm{c}}\right)^{-T} \\
& =\mathbf{T}_{3}^{\mathrm{c}} \operatorname{diag}\left\{\left(\mathbf{C Q}_{\omega}\right)^{\mathrm{H}}(:, i)\left(\mathbf{C Q}_{\omega}\right)^{\mathrm{H}}(:, p)\right\}\left(\mathbf{T}_{3}^{\mathrm{c}}\right)^{-1} \\
& =\mathbf{T}_{3}^{\mathrm{c}}\left(\mathbf{C} \mathbf{Q}_{\omega}\right)^{\mathrm{H}}\left(\mathbf{T}_{3}^{\mathrm{c}}\right)^{-1}
\end{aligned}
$$

Again, we created two joint matrix diagonalization problems for the second-mode slice. Therefore, we can acquire two estimates of $\left(\mathbf{C Q}_{\omega}\right)$ from the joint diagonalization of $\mathbf{S}_{2, i}^{\mathrm{c}, \mathrm{rhs}}$ and $\mathbf{S}_{2, i}^{\mathrm{c}, \mathrm{lhs}}$. Additionally, these joint diagonalization yield estimates for $\mathbf{T}_{1}^{\mathrm{c}}$ and $\mathbf{T}_{3}^{\mathrm{c}}$. Therefore, we can estimate the factor matrices $\mathbf{U}_{1}^{\mathrm{c}} \mathbf{T}_{1}^{\mathrm{c}}=\hat{\boldsymbol{\Gamma}}^{\mathrm{T}}$, and $\mathbf{U}_{3}^{\mathrm{c}} \mathbf{T}_{3}^{\mathrm{c}}=\hat{\mathbf{A}}^{\mathrm{T}}$.

Finally, we have to define the right-hand and left-hand matrices for the first-mode slice. Again, we firstly define the right-hand matrix in the following fashion:

$$
\begin{aligned}
\mathbf{S}_{1, i}^{\mathrm{c}, \mathrm{rhs}} & =\mathbf{S}_{1, i}^{\mathrm{c}}\left(\mathbf{S}_{1, p}^{\mathrm{c}}\right)^{-1} \\
& =\mathbf{T}_{2}^{\mathrm{c}} \operatorname{diag}\left\{\boldsymbol{\Gamma}^{\mathrm{H}}(:, i) \boldsymbol{\Gamma}^{\mathrm{H}}(:, p)\right\}\left(\mathbf{T}_{2}^{\mathrm{c}}\right)^{-1} \\
& =\mathbf{T}_{2}^{\mathrm{c}} \boldsymbol{\Gamma}^{\mathrm{H}}\left(\mathbf{T}_{2}^{\mathrm{c}}\right)^{-1},
\end{aligned}
$$


then we define the left-hand matrix

$$
\begin{aligned}
\mathbf{S}_{1, i}^{\mathrm{c}, \text { lhs }} & =\left(\left(\mathbf{S}_{1, p}^{\mathrm{c}}\right)^{-1} \mathbf{S}_{1, i}^{\mathrm{c}}\right)^{\mathrm{T}}=\left(\mathbf{S}_{1, i}^{\mathrm{c}}\right)^{\mathrm{T}}\left(\mathbf{S}_{1, p}^{\mathrm{c}}\right)^{-T} \\
& =\mathbf{T}_{3}^{\mathrm{c}} \operatorname{diag}\left\{\boldsymbol{\Gamma}^{\mathrm{H}}(:, i) \boldsymbol{\Gamma}^{\mathrm{H}}(:, p)\right\}\left(\mathbf{T}_{3}^{\mathrm{c}}\right)^{-1} \\
& =\mathbf{T}_{3}^{\mathrm{c}} \boldsymbol{\Gamma}^{\mathrm{H}}\left(\mathbf{T}_{3}^{\mathrm{c}}\right)^{-1}
\end{aligned}
$$

Thus, the joint diagonalization problems $\mathbf{S}_{1, i}^{\mathrm{c}, \text { rhs }}$ and $\mathbf{S}_{1, i}^{\mathrm{c}, \text { lhs }}$ for the first-mode slice yield two estimates for $\Gamma$. Furthermore, the joint diagonalization results in estimates for $\mathbf{T}_{2}^{\mathrm{c}}$ and $\mathbf{T}_{3}^{\mathrm{c}}$. Thus, we can estimate the factor matrices $\mathbf{U}_{2}^{\mathrm{c}} \mathbf{T}_{2}^{\mathrm{c}}=\left(\hat{\mathbf{C}} \mathbf{Q}_{\omega}\right)^{\mathrm{T}}$, and $\mathbf{U}_{3}^{\mathrm{c}} \mathbf{T}_{3}^{\mathrm{c}}=\hat{\mathbf{A}}^{\mathrm{T}}$.

Through performing various simulations using all mode slices previously described, we discovered that the right-hand matrices and left-hand matrices of each mode slice yielded similar time-delay estimation to the state-of-the-art CPD-GEVD method for a scenario with one LOS and one NLOS. However, we too discovered that when we have one impinging LOS and two NLOS the right-hand matrix of the third-mode slice have the best performance. Thus, we propose to only use the estimate provided by the joint diagonalization of $\mathbf{S}_{3, i}^{\text {c,rrs }}$. Therefore, our goal is to find $\hat{\mathbf{T}}_{1}^{\mathrm{c}}$ that simultaneously diagonalizes the $N-1$ equations for the right-hand matrix of the second-mode slice. We refer here to the techniques in [21] and [22]. Then, we use $\mathbf{U}_{1}^{c}$ from (3.27) to estimate $\hat{\Gamma}^{\mathrm{T}}$

$$
\mathbf{U}_{1}^{\mathrm{c}} \hat{\mathbf{T}}_{1}^{\mathrm{c}}=\hat{\boldsymbol{\Gamma}}^{\mathrm{T}}
$$

Next, since the noiseless case, according to (2.9) the first-mode unfolding of $\mathcal{Y}$ can be described as

$$
[\mathcal{Y}]_{(1)}^{\mathrm{T}}=\left[\left(\mathbf{C Q}_{\omega}\right) \diamond \mathbf{A}\right] \boldsymbol{\Gamma}
$$

and once $\hat{\boldsymbol{\Gamma}}^{\mathrm{T}}$ is given in (3.45), we define $\mathbf{F}^{\mathrm{c}(2,3)}$ as

$$
\begin{aligned}
\mathbf{F}^{\mathrm{c}(2,3)} & =[\mathcal{Y}]_{(1)}^{\mathrm{T}} \hat{\boldsymbol{\Gamma}}^{+\mathrm{T}}=\left[\left(\mathbf{C} \mathbf{Q}_{\omega}\right)^{\mathrm{T}} \diamond \mathbf{A}\right] \boldsymbol{\Gamma} \hat{\boldsymbol{\Gamma}}^{+\mathrm{T}} \\
& \approx\left[\left(\mathbf{C} \mathbf{Q}_{\omega}\right)^{\mathrm{T}} \diamond \mathbf{A}\right] \in \mathbb{C}^{Q M \times L_{d}} .
\end{aligned}
$$




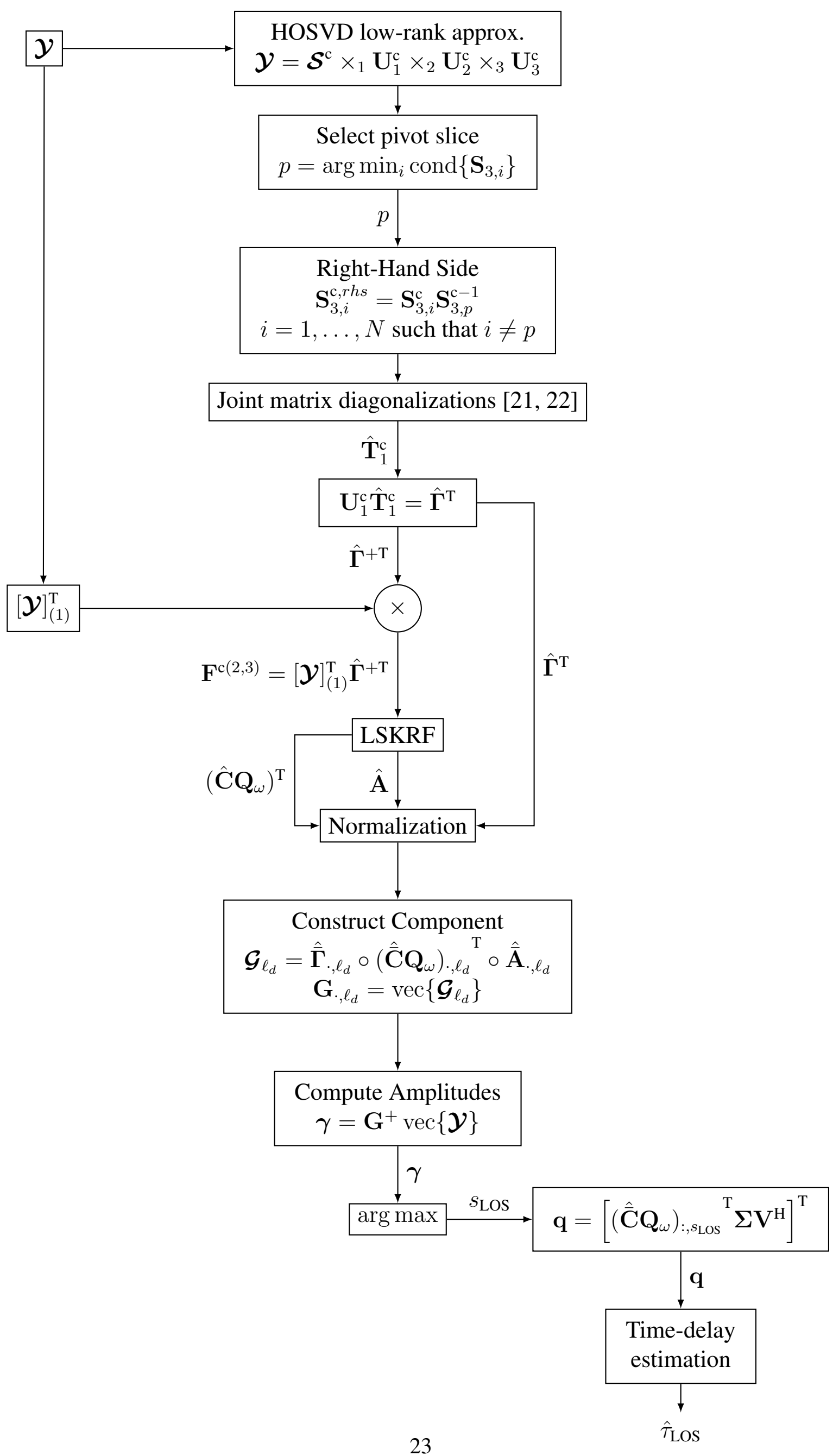

Figure 3.4: Proposed SECSI based time-delay estimation block diagram using the right-hand 
The factor matrices $\left(\hat{\mathbf{C}} \mathbf{Q}_{\omega}\right)^{\mathrm{T}}$ and $\hat{\mathbf{A}}$ can be estimated from (3.47) by applying the Least Squares Khatri-Rao Factorization (LSKRF) [16, 17]. Furthermore, we use the estimated factor matrices to perform the time-delay estimation technique described in Subsection ??

\subsection{Computational Complexity}

In this section, the state-of-the-art HOSVD+FBA+ESPS, DoA/KRF, and CPD-GEVD as well as the proposed HOSVD SECSI method computational complexity is discussed.

The computational complexity is computed in term of FLoating point OPeration (flop) counts. For instance, the computational complexity of a matrix multiplication between two complex matrices, $\mathbf{A} \in \mathbb{C}^{M \times N}$ and $\mathbf{B} \in \mathbb{C}^{N \times L}$ is denoted as $\mathcal{O}(A B)=2 M N L$ [37]. Since unfolding, inverse-unfolding are functions about data representation, we are not considering this operations in the computational complexity. In Subsection 3.3.1 we show the computational complexity of the state-of-the-art HOSVD+FBA+ESPS. Subsection 3.3.2 show the computational complexity of the state-of-the-art DoA/KRF method. Subsection 3.3.3 show the computational complexity of the state-of-the-art CPD-GEVD method. In Subsection 3.3.4 we show the computational complexity of the proposed HOSVD SECSI.

\subsubsection{Complexity of HOSVD+FBA+ESPS}

Since we want to compute $\mathrm{q}_{\mathrm{ESPS}} \in \mathbb{C}^{Q \times 1}$, firstly we compute the FBA of the received tensor $\mathcal{Y}$. The FBA pre-processing step results in the following complexity:

$$
\mathcal{O}(\mathrm{FBA})=2 M^{2} K Q+2(K Q)^{2} M
$$

then, after computing the FBA we compute the ESPS, which yields the following complexity:

$$
\mathcal{O}(\mathrm{ESPS})=[2 M(2 K Q)] L_{s}
$$

afterwards we use the resulting tensor $\mathcal{Z}_{\text {ESPS }}$ to perform the HOSVD rank-one operation. Therefore, the HOSVD complexity is given by:

$$
\begin{aligned}
\mathcal{O}(\text { HOSVD }) & =\left[4 K^{3}+I_{K}\left(8 K^{2}+10 K\right)\right]+\left[4 Q^{3}+I_{Q}\left(8 Q^{2}+10 Q\right)\right] \\
& +\left[4 L_{S}^{3}+I_{L_{S}}\left(8 L_{S}^{2}+10 L_{S}\right)\right]+\left[4 M_{S}^{3}+I_{M_{S}}\left(8 M_{S}^{2}+10 M_{S}\right)\right]
\end{aligned}
$$

where $I_{K}$ is the number of SVD power operations performed when using the first-mode unfolding of tensor $\mathcal{Z}_{\mathrm{ESPS}}, I_{Q}$ is the number of SVD power operations performed when using the second-mode unfolding, $I_{M_{S}}$ is the number of SVD power operations performed when using the third-mode unfolding, $I_{L_{S}}$ is the number of SVD power operations performed 
when using the fourth-mode unfolding.

Then, in order to compute the vector $\mathrm{q}_{\mathrm{ESPS}}$, we have the following complexity:

$$
\mathcal{O}\left(\mathbf{q}_{\mathrm{ESPS}}\right)=4 K Q L_{S} M_{S}+4 L_{S} K Q M_{S}+4 L_{S} K Q M_{S}+4 L_{S} K Q M_{S}
$$

Moreover, we can define the total computational complexity of the state-of-the-art HOSVD+FBA+ESPS methods as:

$$
\mathcal{O}\left(\mathrm{HOSVD}+\mathrm{FBA}+\mathrm{ESPS}=\mathcal{O}(\mathrm{FBA})+\mathcal{O}(\mathrm{ESPS})+\mathcal{O}(\mathrm{HOSVD})+\mathcal{O}\left(\mathrm{q}_{\mathrm{ESPS}}\right)\right.
$$

\subsubsection{Complexity of DoA/KRF}

Additionally, we compute the computational complexity of the state-of-the-art DoA/KRF. Therefore, once the first step performs the ESPRIT operation, we define the ESPRIT computational complexity as follows:

$$
\begin{aligned}
\mathcal{O}(\text { ESPRIT }) & =2 K Q M^{2}+2 K Q^{2} M+I\left(4 K Q^{2}+5 K Q+4 M^{2}+5 M\right) \\
& +2\left[2(M-1) L_{d}^{2}\right]+\frac{5}{3}(M-1)^{3}+L_{d}^{3}
\end{aligned},
$$

where $I$ is the number of SVD power operations. Afterwards, the Khatri-Rao Factorization computational complexity can be described as:

$$
\mathcal{O}(\mathrm{KRF})=2 L_{d} K Q^{2}+2 L_{d} K^{2} Q+L_{d} I\left(4 K^{2}+5 K+4 Q^{2}+5 Q\right) .
$$

Then, the DoA/KRF method normalizes the estimated factor matrices, and computes the amplitudes. Therefore, the normalization and amplitude estimation has a computational complexity:

$$
\mathcal{O}(\mathrm{NORM}+\mathrm{AMP})=L_{d}(K Q+K Q M)+2 L_{d}^{2} K Q M+\frac{5}{3} L_{d}^{3}+2 L_{d}^{2} K Q M .
$$

Furthermore, we can define the total computational complexity by summing the computed complexities with other minor operations used in the the DoA/KRF method:

$$
\begin{aligned}
\mathcal{O}(\mathrm{DoA} / \mathrm{KRF}) & =\mathcal{O}(\mathrm{ESPRIT})+\mathcal{O}(\mathrm{KRF})+\mathcal{O}(\mathrm{NORM}+\mathrm{AMP}) \\
& +2 L_{d}^{2} M+\frac{5}{3} L_{d}^{3}+2 L_{d}^{2} M+2 L_{d} M Q+2 Q+L_{d}^{3}
\end{aligned}
$$




\subsubsection{Complexity of CPD-GEVD}

Similarly to the HOSVD+FBA+ESPS, the state-of-the-art CPD-GEVD show the same computational complexity as (3.50) when performing the HOSVD step. Furthermore, we compute the LSKRF computational complexity as follows:

$$
\mathcal{O}(\mathrm{LSKRF})=2 L_{d} M Q^{2}+2 L_{d} M^{2} Q+L_{d} I\left(4 M^{2}+5 M+4 Q^{2}+5 Q\right) .
$$

Then, we can define the final computational complexity by summing the computed complexities with the steps that compute the GEVD, factor matrix $\hat{\Gamma}$, factor matrix $\mathbf{F}^{(2,3)}$, and least square as follows:

$$
\begin{aligned}
\mathcal{O}(\mathrm{CPD}-\mathrm{GEVD}) & =\mathcal{O}(\mathrm{HOSVD})+\mathcal{O}(\mathrm{LSKRF})+\mathcal{O}(\mathrm{NORM}+\mathrm{AMP}) \\
& +L_{d}^{3}+2 K L_{d}^{2}+2 Q M K L_{d}+2 L_{d} K Q+4 K M Q
\end{aligned}
$$

\subsubsection{Complexity of HOSVD SECSI}

Since we decided to use only the right-hand matrix of the third-mode unfolding to perform time-delay estimation, we only calculated the computational complexity of HOSVD SECSI for the first factor estimate. Similarly to the state-of-the-art CPD-GEVD, the HOSVD low-rank approximation step of proposed HOSVD SECSI shows the same computational complexity as Equation (3.50). Moreover, after HOSVD low-rank approximation we compute the computational complexity of constructing the third-mode slice as:

$$
\mathcal{O}(3-\mathrm{MODE})=M L_{d}^{3}
$$

Then, we define the complexity of the conditional operation as:

$$
\mathcal{O}(\mathrm{COND})=4 L_{d}^{3}+I\left(8 L_{d}^{2}+10 L_{d}\right)
$$

where $I$ is the number of SVD power operations. Then, we define the computational complexity of computing the right-hand matrix as:

$$
\mathcal{O}(\text { RIGHT-HAND })=N L_{d}^{3}
$$

where $N$ is the number of slices. Furthermore, we define the joint diagonalization computa- 
tion complexity as:

$$
\mathcal{O}(\text { JOINTDIAG })=J\left(4 L_{d}^{2}+16 L_{d}^{2}+\frac{10}{3} L_{d}\right)
$$

where $J$ is the number of iterations. Moreover, the LSKRF has the same computational complexity as (3.57). Finally, we compute the total computational complexity by combining the computed complexities with inverse, pseudo-inverse, and factor matrices estimation computational complexities as follows:

$$
\begin{aligned}
\mathcal{O}(\text { SECSI }) & =\mathcal{O}(\text { HOSVD })+\mathcal{O}(3-\text { MODE })+\mathcal{O}(\text { LSKRF })+\mathcal{O}(\text { COND }) \\
& +\mathcal{O}(\text { RIGHT-HAND })+\mathcal{O}(\text { JOINTDIAG })+\mathcal{O}(\text { NORM }+ \text { AMP }) \\
& +\frac{10}{3} L_{d}^{3}+6 K L_{d}^{2}+2 L_{d} K Q
\end{aligned}
$$

\subsection{Results}

In this section we present the time-delay estimation results obtained through several simulations. In Subsection 3.4.1 we present simulations results using the state-of-the-art scenario used by [23]. Subsection 3.4.2 presents our proposed scenario and describes the simulations results for the proposed scenario.

\subsubsection{State-of-the-art Scenario}

The simulation scenario consists of a left centro-hermitian uniform linear array with $M=8$ elements and half-wavelength $\Delta=\lambda / 2$ spacing, as proposed by [23]. The /A code is transmitted from the satellite with $P R N=17$ with a carrier frequency $f_{s}=1575.42 \mathrm{MHz}$. the C/A code has a bandwidth $B=1.023 \mathrm{MHz}$ with $N=2046$ samples collected every $K$-th epoch during $K=30$ epochs. Moreover, LOS and NLOS components are generated with an azimuth angle difference $\Delta \phi=60^{\circ}$. Furthermore, when we have $L_{d}=2$ impinging signals we have a NLOS time-delay $\tau_{\mathrm{NLOS}_{1}}=\tau_{\mathrm{LOS}}+\Delta \tau$ and when we have $L_{d}=3$ impinging signals we have a second NLOS time-delay $\tau_{\mathrm{NLOS}_{2}}=$ $\tau_{\mathrm{NLOS}_{1}}+\Delta \tau$. In order to perform the SPS/ESPS the antenna array is separated into $L_{S}=5$ subarrays with $M_{S}=4$ elements each. The carrier-to-noise ratio is $C / N_{0}=48 \mathrm{~dB}-\mathrm{Hz}$, resulting in a pre-correlation $S N R=C / N_{0}-10 \log _{10}(2 B) \approx-15 \mathrm{~dB}$. Given the processing gain $G=10 \log _{10}(B t) \approx 30 \mathrm{~dB}$, we have a post-correlation $S N R=S N R+G \approx 15 \mathrm{~dB}$. Moreover, we have a signal to multipath ratio $\mathrm{SMR}=5 \mathrm{~dB}$ for the scenario where we have only one NLOS. Additionally, when we have two NLOS we have two different signal to multipath ratio for each NLOS thus we have a $\mathrm{SMR}_{1}=5 \mathrm{~dB}$ for the first NLOS and a $\mathrm{SMR}_{2}=10 \mathrm{~dB}$ for the second NLOS. 
In this section, we performed 2000 Monte Carlo (MC) simulations to compare all approaches in terms of the Root Mean-Squared Error (RMSE) of the time-delay estimation of the LOS components considering the state-of-the-art HOSVD, DoA/KRF and CPD-GEVD based approaches, the proposed HOSVD SECSI approach, and the ideal case, filtering supposing known $\tilde{\Gamma}$ and $\mathbf{A}$.

In Figure 3.5 we show the results to the state-of-the-art scenario where we performed $2000 \mathrm{MC}$ for the state-of-the-art tensor-based time-delay estimation methods and for the proposed HOSVD SECSI. Observe that the HOSVD+FBA+ESPS shows the worst performance by having a peak error of about $0.83 \mathrm{~m}$ at $\Delta \tau=0.5 T_{c}$. Note that the state-of-the-art DoA/KRF and CPD-GEVD methods, and the proposed SECSI method have similar performance. However, the SECSI method displays a worst performance than the state-of-the-art CPD-GEVD when signals are strongly correlated

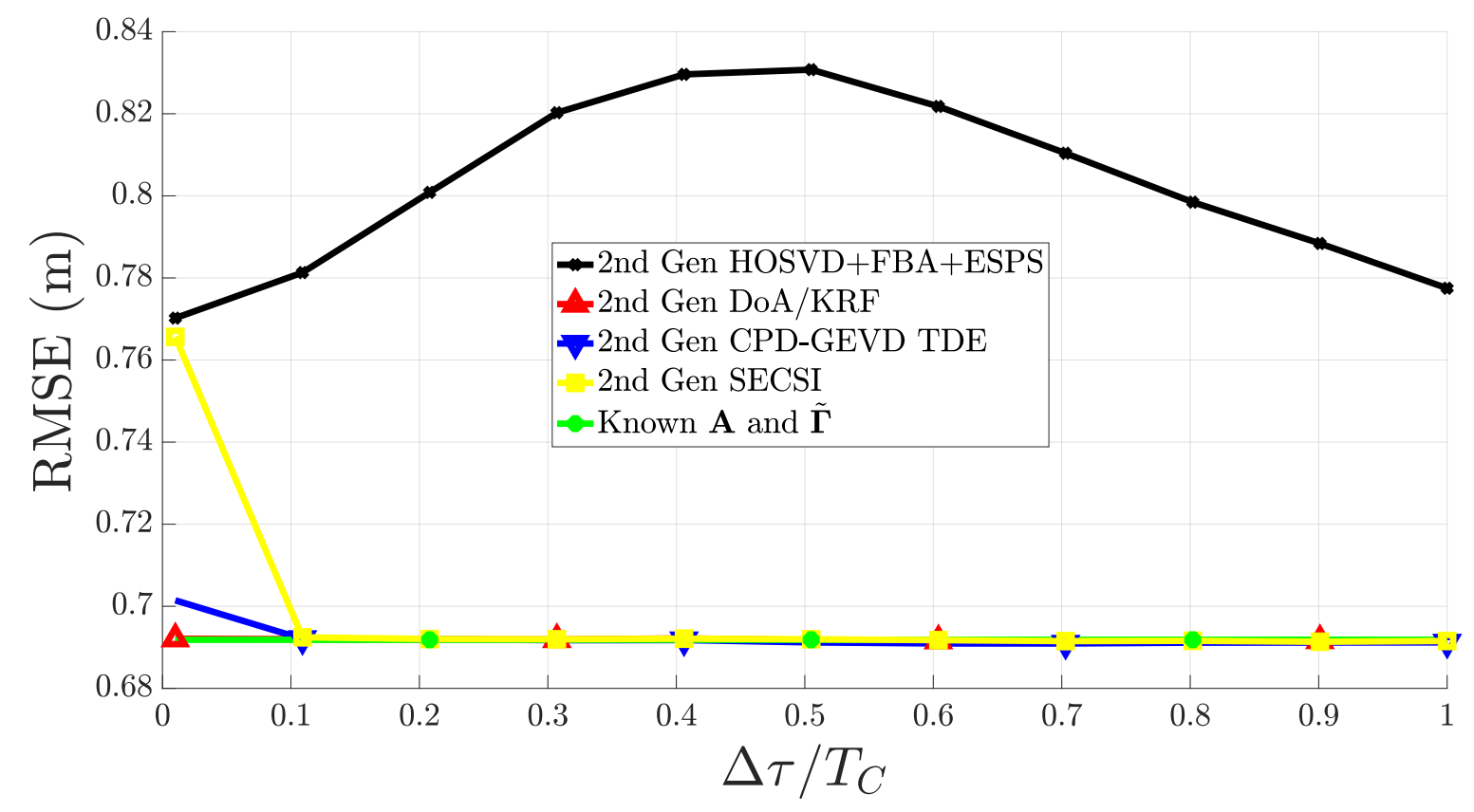

Figure 3.5: Simulation with $\left(L_{d}=2\right)$ signals for state-of-the-art 2nd Generation with $N=$ 2046 samples. Code samples are collected during $K=30$ epochs with $M=8$ antenna.

In Figure 3.6 we show the results to the state-of-the-art scenario where we performed 2000 MC for the state-of-the-art tensor-based time-delay estimation methods and for the proposed HOSVD SECSI. Observe that the CPD-GEVD shows the worst performance by having a peak error of about $7 \mathrm{~m}$ at $\Delta \tau=0.1 T_{c}$. Note that the state-of-the-art DoA/KRF and the proposed SECSI method have similar performance. However, the SECSI method displays a worst performance than the state-of-the-art DoA/KRF when signals are strongly correlated. 


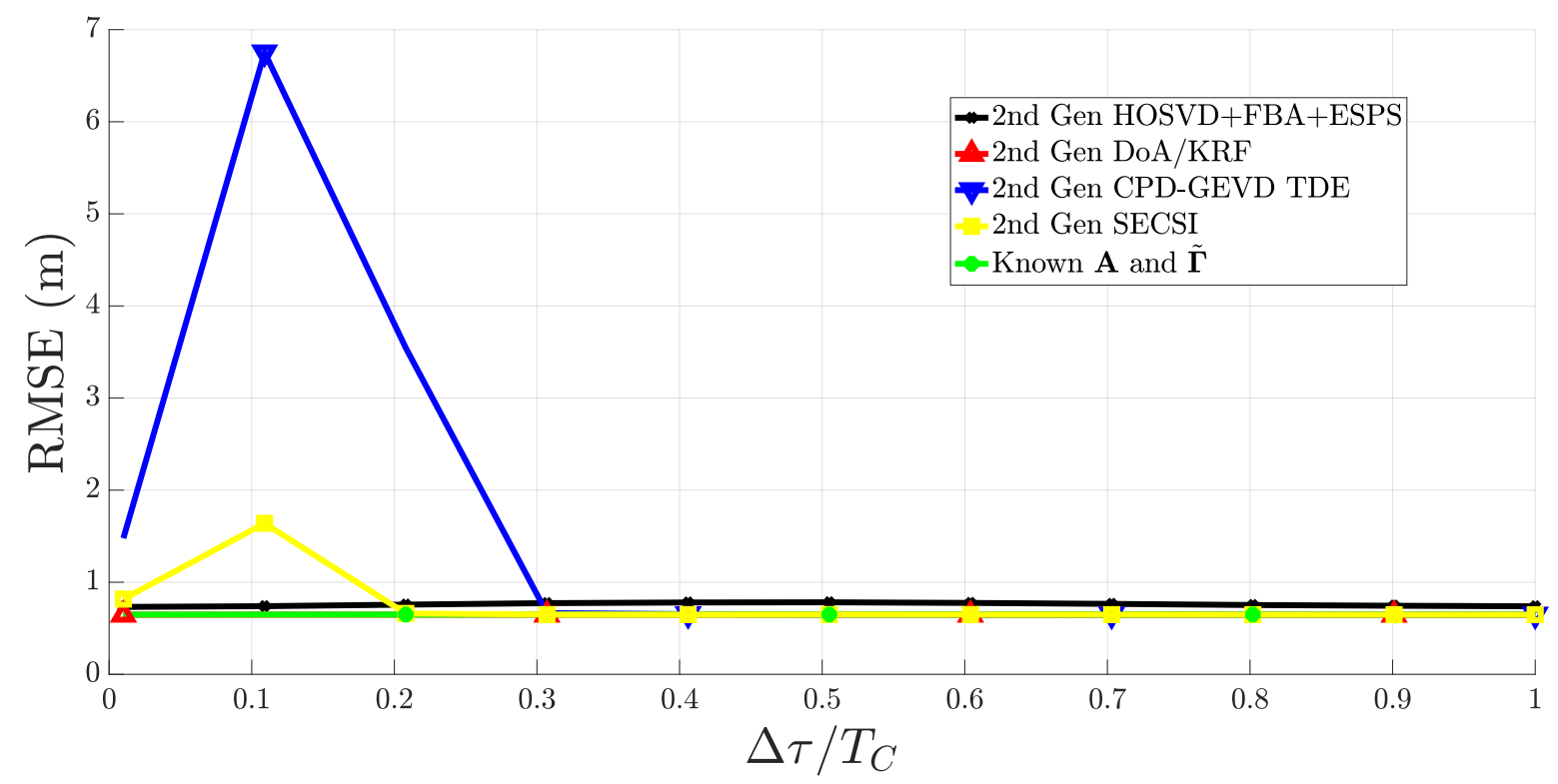

Figure 3.6: Simulation with $\left(L_{d}=3\right)$ signals for state-of-the-art 2nd Generation with $N=$ 2046 samples. Code samples are collected during $K=30$ epochs with $M=8$ antenna.

\subsubsection{Proposed scenario}

Similarly to [6], the simulation scenario consists of a left centro-hermitian uniform linear array with $M=8$ elements and half-wavelength $\Delta=\lambda / 2$ spacing. Both L1C pilot channel and C/A code are transmitted from the satellite with $P R N=17$ with a carrier frequency $f_{s}=1575.42 \mathrm{MHz}$. The simulation considers using the modulated L1C pilot channel with a total period $t_{3 \mathrm{rd}}=10 \mathrm{~ms}$ with a bandwidth $B_{3 \mathrm{rd}}=12.276 \mathrm{MHz}$, the higher bandwidth is due to TMBOC modulation [6]. In addition, to perform a fair comparison, [6] proposed an increasing in the C/A code bandwidth to $B_{2 n d}=B_{3 \mathrm{rd}}$. Both L1C pilot channel and C/A code samples are collected every $K$-th epoch during $K=30$ epochs with each epoch during $10 \mathrm{~ms}$ [6]. Thus, once C/A code has a total period of $t_{2 \mathrm{nd}}=1 \mathrm{~ms}$, each epoch of C/A code collects $10 \mathrm{~ms}$ of data. Therefore, $N=245520$ samples were collected of both L1C pilot and C/A code per epoch.

Furthermore, the LOS and NLOS components are generated with an azimuth angle difference $\Delta \phi=60^{\circ}$. In similar fashion to the state-of-the-art scenario, when we have $L_{d}=2$ signal components we define the first NLOS time-delay as $\tau_{\mathrm{NLOS}_{1}}=\tau_{\mathrm{LOS}}+\Delta \tau$ and when we have $L_{d}=3$ signal components we define a second NLOS time-delay $\tau_{\mathrm{NLOS}_{2}}=\tau_{\mathrm{NLOS}_{1}}+\Delta \tau$. In the same fashion as [6], in order to perform the SPS/ESPS the antenna array is separated into $L_{S}=5$ subarrays with $M_{S}=4$ elements each. The carrier-to-noise ratio is $C / N_{0}=48$ $\mathrm{dB}-\mathrm{Hz}$, resulting in a pre-correlation $S N R_{\text {pre }_{\text {2nd }}}=C / N_{0}-10 \log _{10}\left(2 B_{2 \text { nd }}\right) \approx-25.10 \mathrm{~dB}$ for the second generation GPS and a $S N R_{\text {pre }_{3 \mathrm{rd}}}=C / N_{0}-10 \log _{10}\left(2 B_{3 \mathrm{rd}}\right) \approx-25.10 \mathrm{~dB}$ for third generation GPS. Given the processing gain $G_{3 \mathrm{rd}}=10 \log _{10}\left(B_{3 \mathrm{rd}} t_{3 \mathrm{rd}}\right) \approx 50.9 \mathrm{~dB}$ for third generation GPS and, once the C/A code is collected during $10 \mathrm{~ms}$, the second generation GPS has a processing gain $G_{2 \text { nd }}=10 \log _{10}\left(B_{2 \text { nd }} t_{3 \mathrm{rd}}\right) \approx 50.9 \mathrm{~dB}$, thus we have a 
post-correlation $S N R_{\text {post }_{\text {nd }}}=S N R_{\text {pre }}+G_{2 \text { nd }} \approx 25 \mathrm{~dB}$ for the second generation GPS and a post-correlation $S N R_{\text {post }_{3 \mathrm{rd}}}=S N R_{\text {pre }}+G_{3 \mathrm{rd}} \approx 25 \mathrm{~dB}$ for the third generation GPS. Furthermore, we have a signal to multipath ratio SMR $=5 \mathrm{~dB}$ both GPS generations for the scenario that we have only one NLOS. Additionally, when we have two NLOS we have two different signal to multipath ratio for each NLOS thus we have a $\mathrm{SMR}_{1}=5 \mathrm{~dB}$ for the first NLOS and $\mathrm{SMR}_{2}=10 \mathrm{~dB}$ for the second NLOS.

Additionally, similarly to [23], besides the simulation using a perfect array we added errors in the antenna array geometry in order to distort the array $x$ and $y$ positions by using a normal distribution $\sim \mathcal{N}\left(0, \sigma^{2}\right)$. The standard deviation is computed in terms of the probability $p=\mathrm{P}(e>\lambda / 2)$ where the error exceeds a half wavelength. Furthermore, we fix the relative delay $\Delta \tau$ at $0.5 T_{c}$ while varying the error probability $p$ from $10^{-6}$ to $10^{-1}$.

Herein we performed 2000 Monte Carlo simulations to compare all approaches in terms of the Root Mean-Squared Error (RMSE) of the time-delay estimation of the LOS components considering the state-of-the-art HOSVD, DoA/KRF and CPD-GEVD based approaches, the proposed HOSVD SECSI approach, and the ideal case, filtering supposing known $\tilde{\Gamma}$ and A. Additionally, we compare the HOSVD SECSI method with the HOOI SECSI method. The HOOI SECSI method simply substitutes the HOSVD low-rankapproximation step by a HOOI low-rank approximation. The results for the MC simulation for the second generation GPS are presented in Subsection 3.4.2.1 while in Subsection 3.4.2.2 we present the results of the MC simulation for the third generation GPS. In Subsection 3.4.2.3 we present the results for the MC simulations when position errors are added to the antenna array. Subsection 3.4.2.4 presents the results for MC simulations when varying the NLOS DoA.

\subsubsection{Second Generation GPS Time-Delay Estimation}

In this section we present simulation results for the state-of-the-art second generation GPS and the proposed second generation GPS using the proposed HOSVD SECSI based time-delay estimation method. Hereby we show the results of two scenarios. In the first scenario both state-of-the-art techniques and SECSI methods suppose an antenna array with $L_{d}=2$ impinging signals while in the second scenario an antenna array with $L_{d}=3$ impinging signals is considered.

Once, similarly to the SECSI decomposition, the CPD-GEVD can produce six different factor estimates for different combinations of the GEVD step, we compare each estimate generated by the CPD-GEVD method with the estimates generated by the SECSI method. Furthermore, in addition to the proposed SECSI method, we performed simulations using the HOOI low-rank approximation instead of the HOSVD low-rank approximation. Thus, in Figure 3.7 we show the result for the state-of-the-art CPD-GEVD based time-delay estimation, which displays an error of about $0.0793 \mathrm{~m}$ when $\Delta \tau>0.1 T_{c}$. Still, in Figure 3.7 we show that the first factor estimate from the SECSI methods match the state-of-the-art 
performance by having an error of about $0.793 \mathrm{~m}$. Note that both both CPD-GEVD and HOSVD SECSI fail to estimate the time-delay error when $\Delta \tau<0.2 T_{c}$ while the HOOI SECSI outputs an error at of approximately $0.796 \mathrm{~m} \Delta \tau=0.01 T_{c}$ and matches the error of CPD-GEVD and HOSVD SECSI when $\Delta \tau=0.2 T_{c}$. The error when having a small delay difference occurs due to the high correlation between LOS and NLOS.

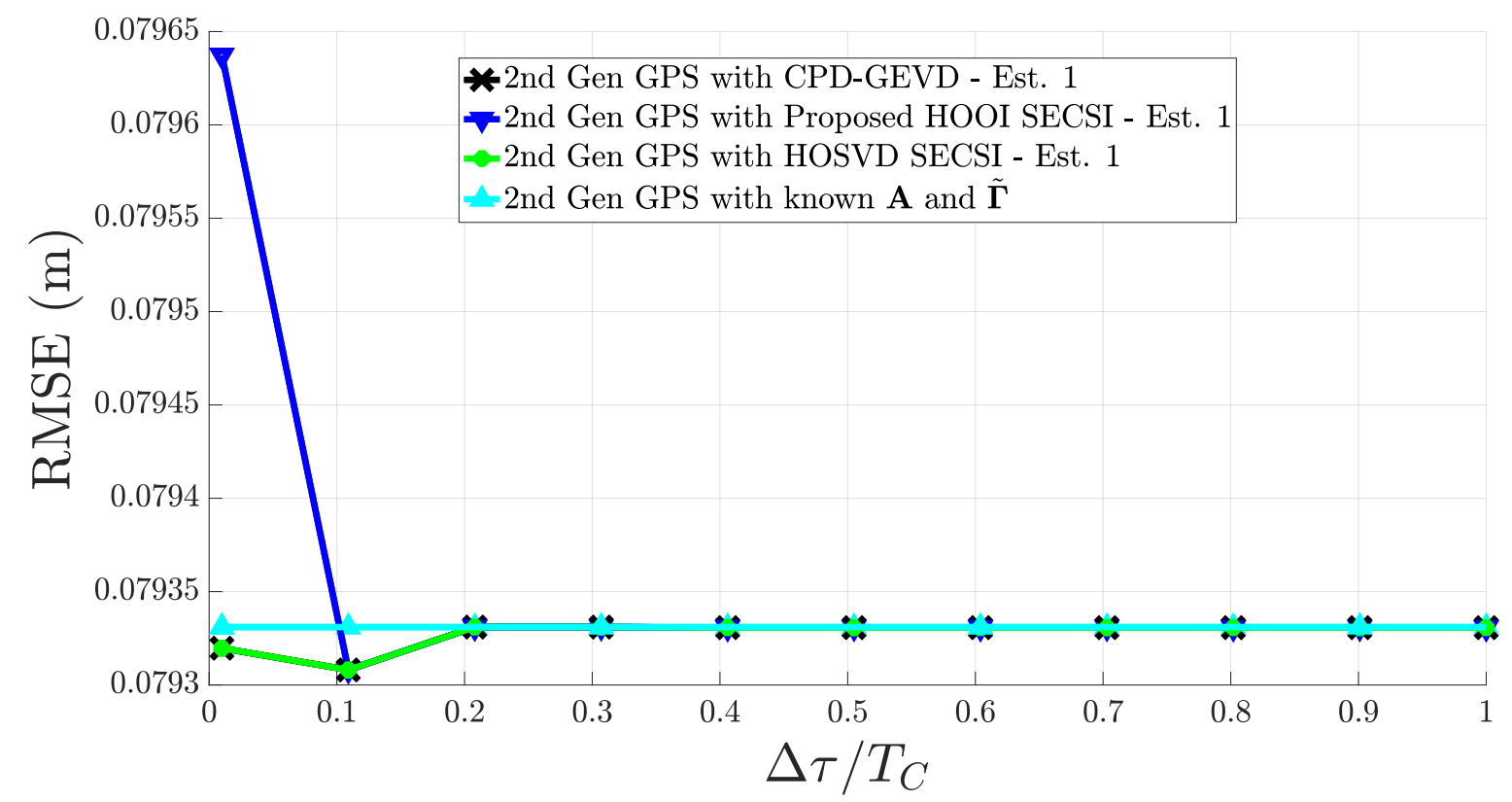

Figure 3.7: State-of-the-art CPD-GEVD, HOSVD SECSI, HOOI SECSI, and proposed SECSI method simulations using the first factor estimate with $M=8$ antennas and $L_{d}=2$ impinging signal. In both cases code samples are collected during $K=30$ epochs, and have $N=245520$ samples.

In Figure 3.8 we use the second factor estimate from both CPD-GEVD, HOSVD SECSI, and HOOI SECSI methods. Note that while the CPD-GEVD perform the time-delay estimation with a peak error of about $0.15 \mathrm{~m}$ at $\Delta \tau=0.01 T_{c}$ and and keeps the error below $0.13 \mathrm{~m}$ when $\Delta \tau>0.1 T_{c}$, the HOSVD SECSI and HOOI SECSI methods present a error of approximately $0.0793 \mathrm{~m}$. 


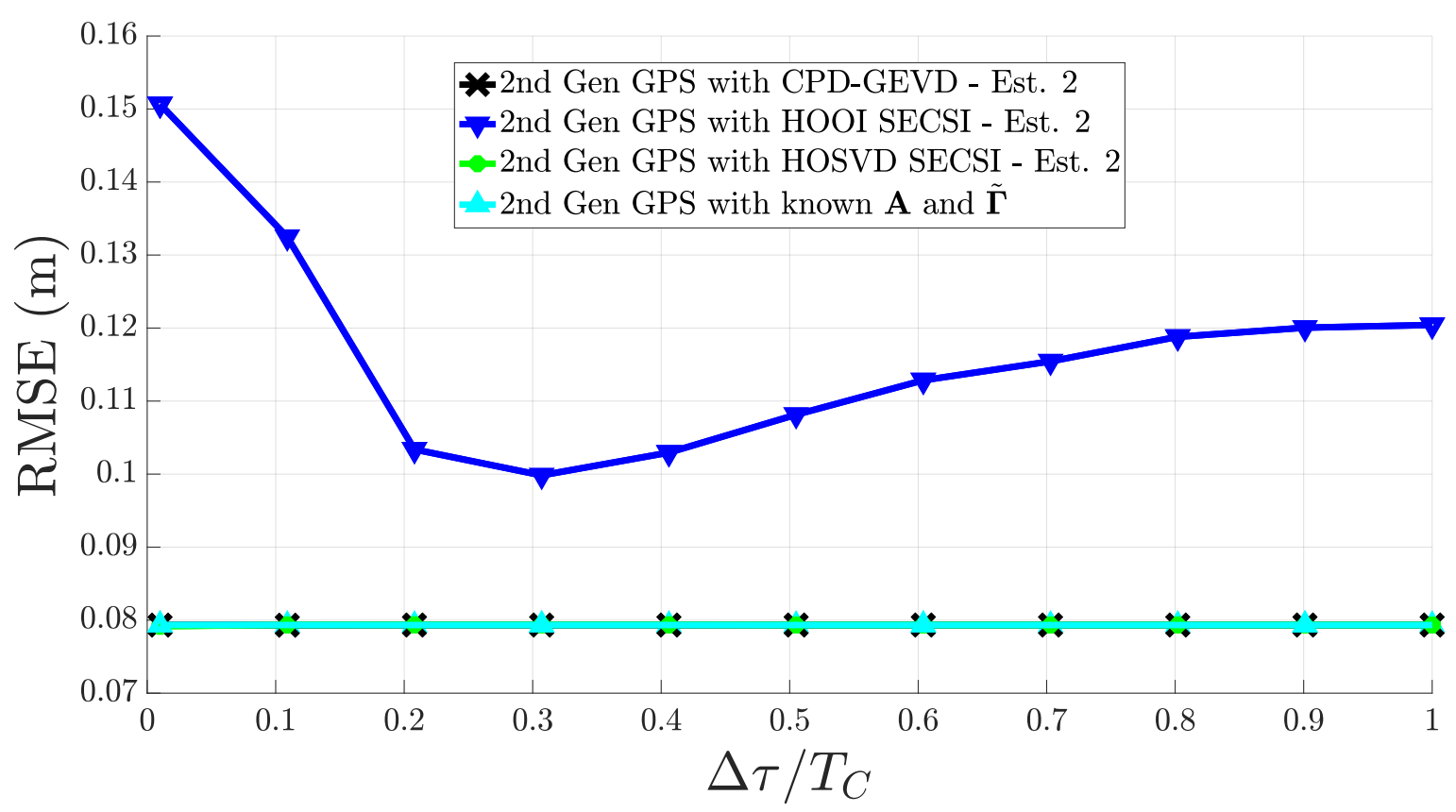

Figure 3.8: State-of-the-art CPD-GEVD, HOSVD SECSI, HOOI SECSI, and proposed SECSI method simulations using the second factor estimate with $M=8$ antennas and $L_{d}=2$ impinging signal. In both cases code samples are collected during $K=30$ epochs, and have $N=245520$ samples.

In Figure 3.9 we use the third factor estimate from both CPD-GEVD, HOOI SECSI, and HOSVD methods. Observe that the third factor estimate matches the first factor estimate show in Figure 3.7 with both state-of-the-art CPD-GEVD, HOSVD SECSI, and HOOI SECSI only successfully estimating the time-delay when $\Delta \tau>0.1 T_{c}$.

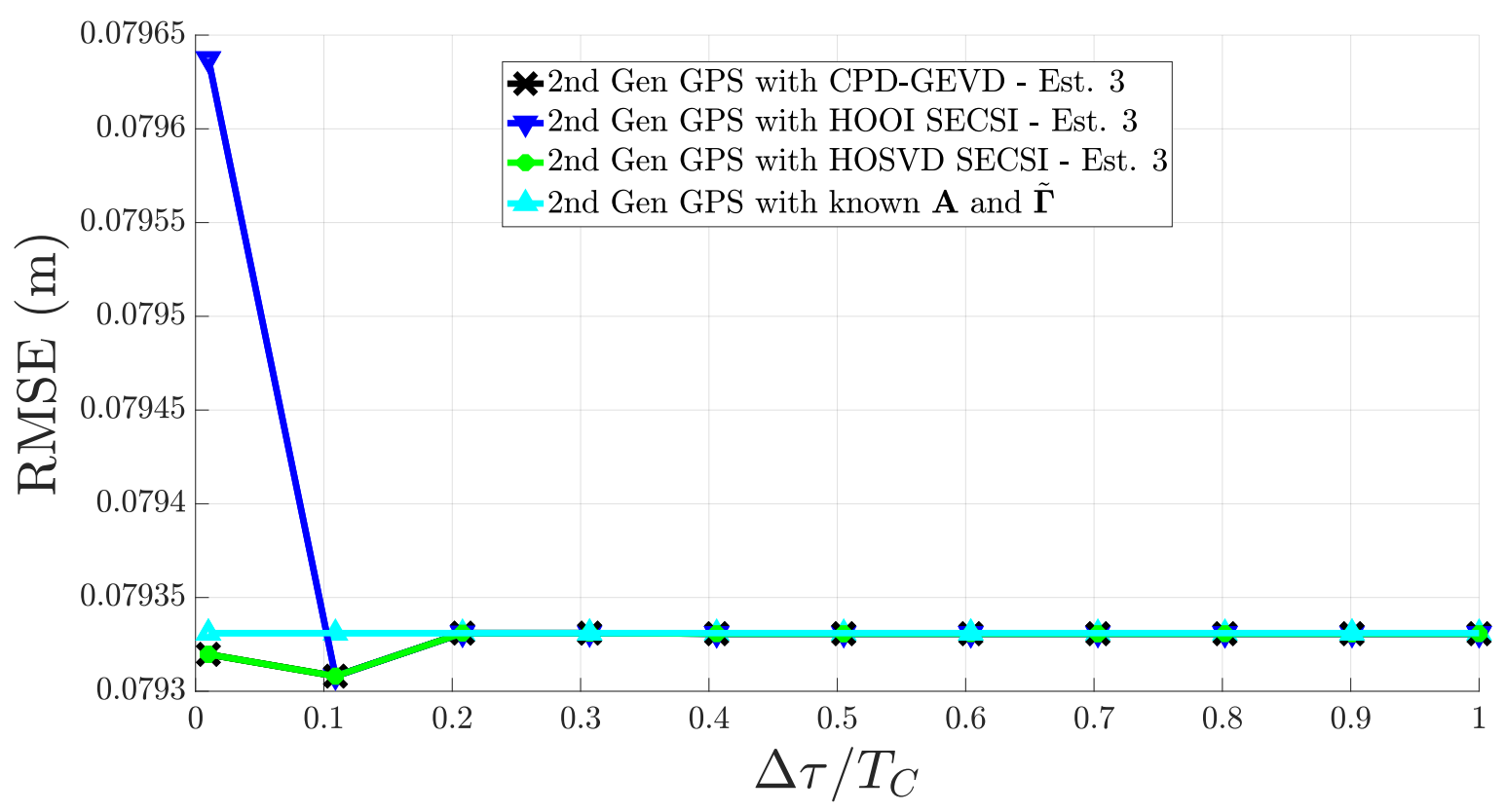

Figure 3.9: State-of-the-art CPD-GEVD, HOSVD SECSI, HOOI SECSI, and proposed SECSI method simulations using the third factor estimate with $M=8$ antennas and $L_{d}=2$ impinging signal. In both cases code samples are collected during $K=30$ epochs, and have $N=245520$ samples. 
Figure 3.10 displays the simulation results when using the fourth factor estimate from CPD-GEVD, HOOI SECSI, and HOSVD SECSI. Note that both HOSVD SECSI, HOOI SECSI, and CPD-GEVD have a larger error when the LOS and NLOS are more correlated. However, when using the forth factor estimate the HOOI SECSI presents a better result when $\Delta \tau<0.1 T_{c}$. Furthermore, the HOOI SECSI presents a worst result when compared with CPD-GEVD at $\Delta \tau>0.1 T_{c}$.

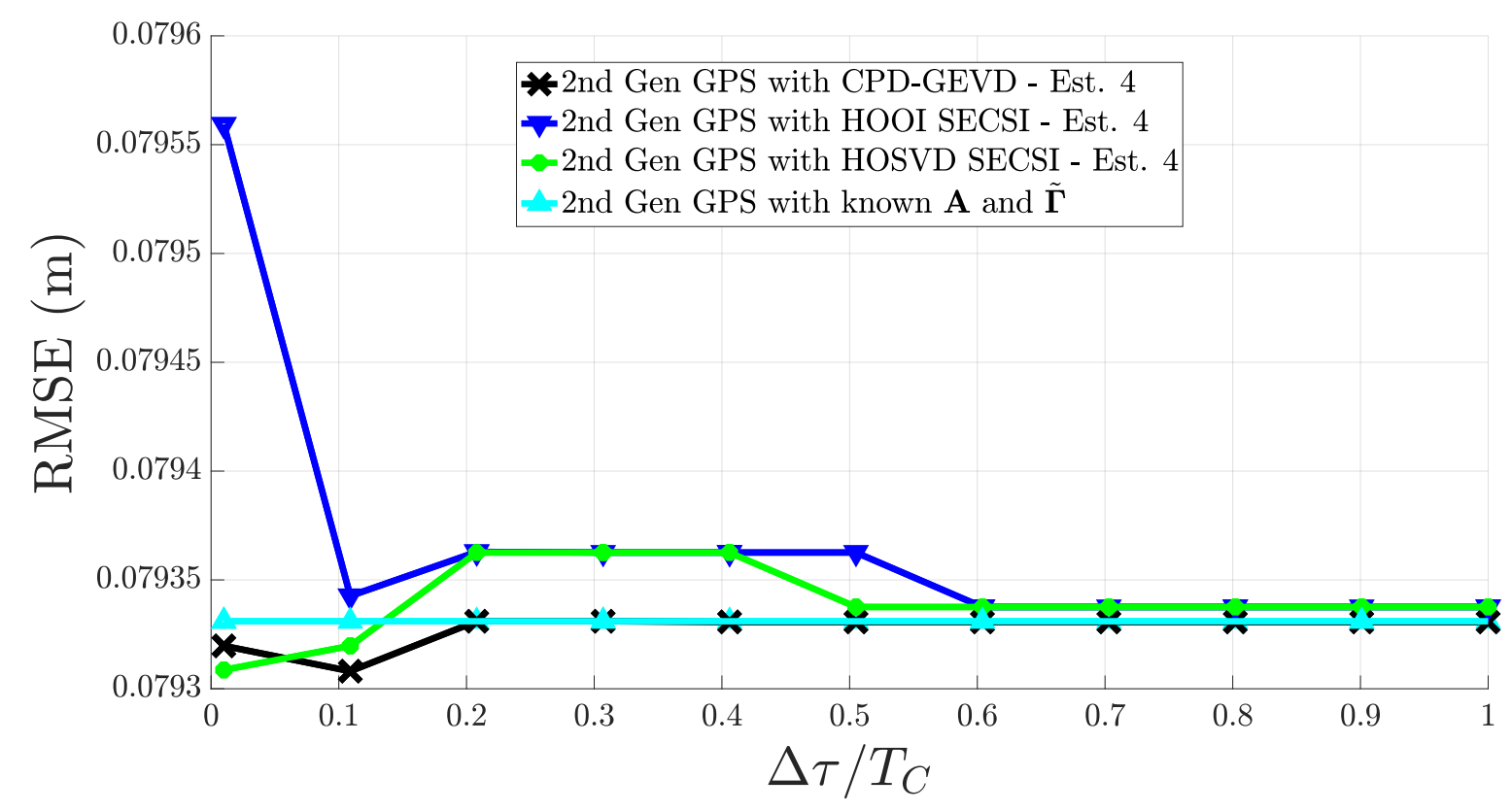

Figure 3.10: State-of-the-art CPD-GEVD, HOSVD SECSI, HOOI SECSI, and proposed SECSI method simulations using the fourth factor estimate with $M=8$ antennas and $L_{d}=2$ impinging signal. In both cases code samples are collected during $K=30$ epochs, and have $N=245520$ samples.

Figure 3.11 show the simulation results when using the fifth factor estimates from CPDGEVD, HOOI SECSI, and HOSVD SECSI. Observe that the CPD-GEVD completely fails on performing time-delay estimation and displays an seemingly linear error. Note that both HOSVD SECSI and HOOI SECSI have similar performance by achieving an overall error of approximately $0.0793 \mathrm{~m}$. 


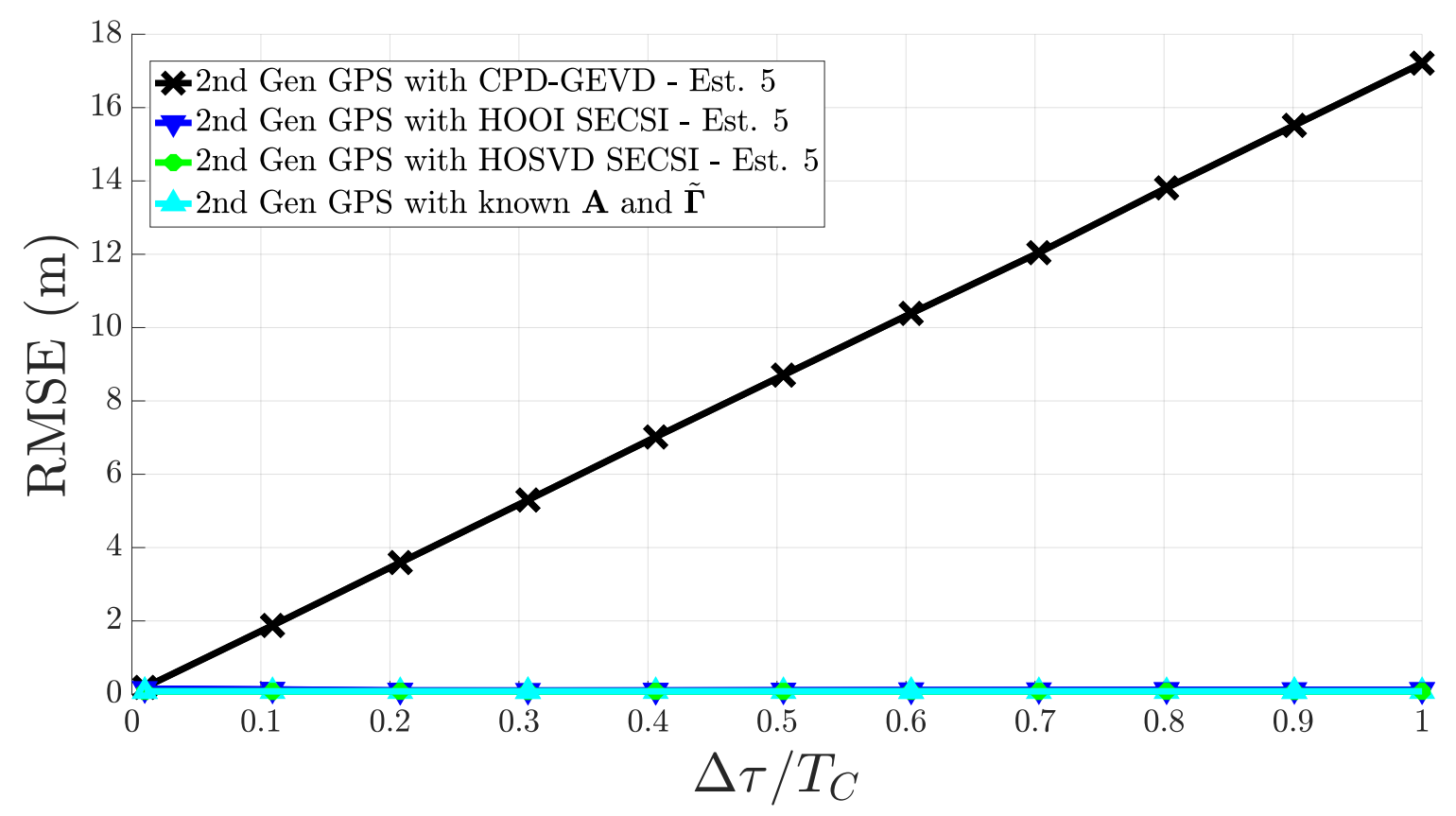

Figure 3.11: State-of-the-art CPD-GEVD, HOSVD SECSI, HOOI SECSI, and proposed SECSI method simulations using the fifth factor estimate with $M=8$ antennas and $L_{d}=2$ impinging signal. In both cases code samples are collected during $K=30$ epochs, and have $N=245520$ samples.

Finally, Figure 3.12 use the sixth factor estimates. Note that the CPD-GEVD completely fails on performing time-delay estimation and displays a linear error. Furthermore, the HOOI SECSI, and HOSVD SECSI methods, have similar performance by achieving an error of about $0.0793 \mathrm{~m}$. 


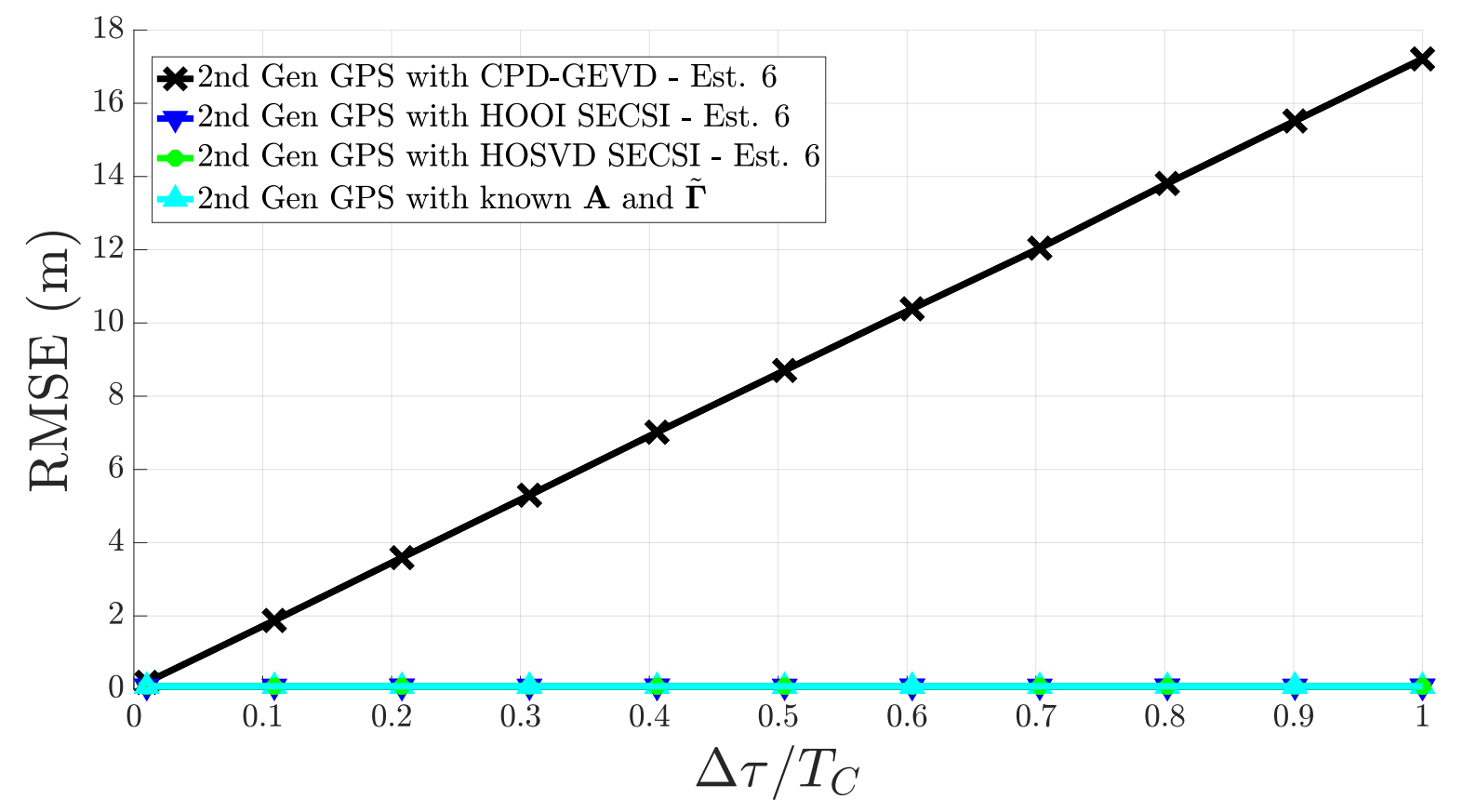

Figure 3.12: State-of-the-art CPD-GEVD, HOSVD SECSI, HOOI SECSI, and proposed SECSI method simulations using the sixth factor estimate with $M=8$ antennas and $L_{d}=2$ impinging signal. In both cases code samples are collected during $K=30$ epochs, and have $N=245520$ samples.

Once we show from Figures 3.7 through 3.12 that the the CPD-GEVD presents the best results when using the first and third factor estimates, we decide to keep the standard CPDGEVD decomposition by using the first factor estimate. Once we show that the all SECSI estimate display the similar performance and the process is similar to the state-of-the-art CPD-GEVD, we decided to pick the first factor estimate from the SECSI in order to perform time-delay estimation. Therefore, in Figure 3.13 the state-of-the-art HOSVD+FBA+ESPS, DoA/KRF, and CPD-GEVD are compared with the SECSI based solution using the first factor estimate. Both state-of-the-art methods and the SECSI method are equipped with an antenna array. Note that the state-of-the-art HOSVD+FBA+ESPS presents the worst result with a peak error of about $0.28 \mathrm{~m}$ at $\Delta \tau=0.6 T_{c}$. Thus, we show that the state-of-the-art DoA/KRF and CPD-GEVD and the SECSI method considerably outperform the state-ofthe-art HOSVD+FBA+ESPS by having an approximately constant error of around $0.079 \mathrm{~m}$. 


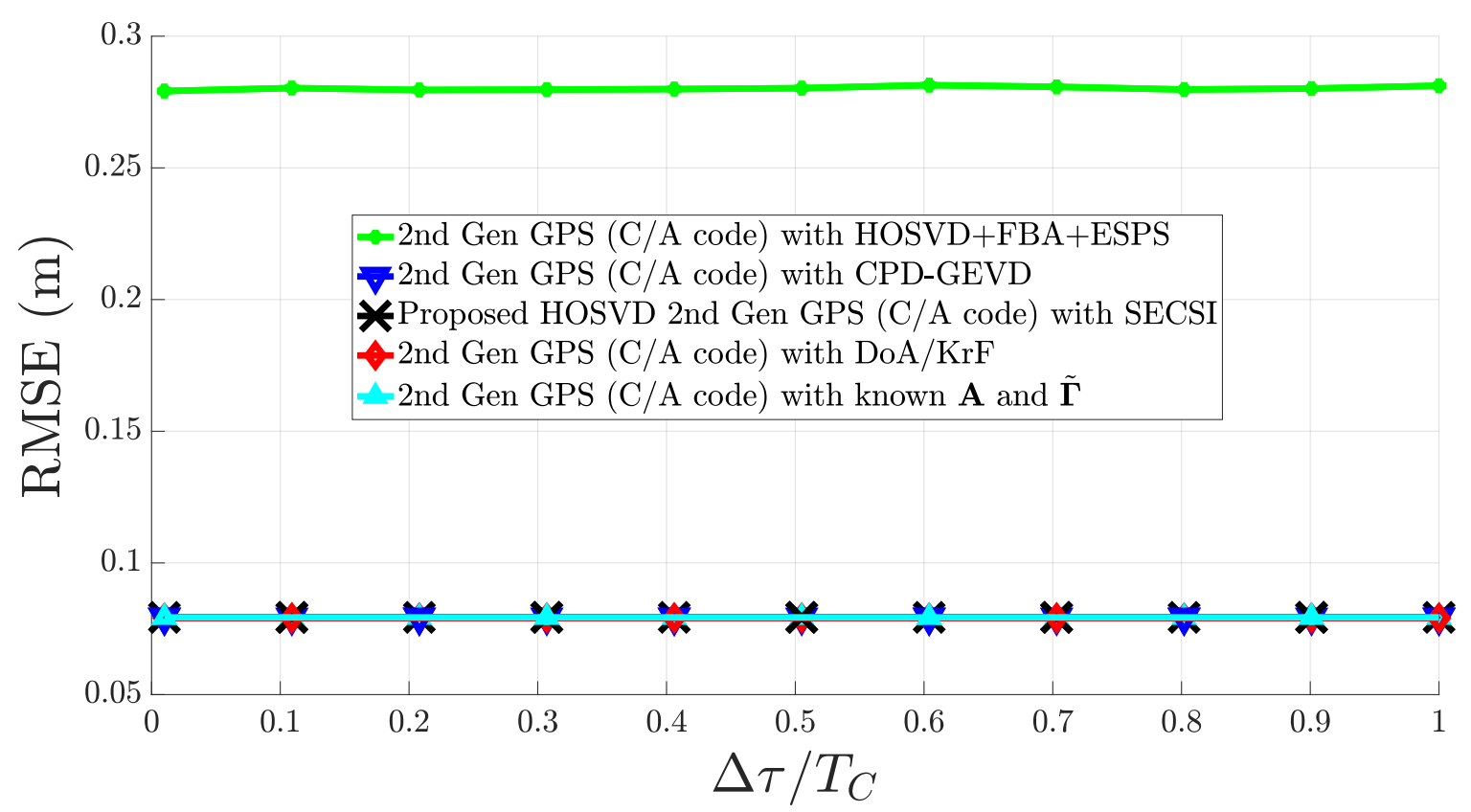

Figure 3.13: State-of-the-art techniques and proposed SECSI method simulation with $M=8$ antennas and $L_{d}=2$ impinging signal. In both cases code samples are collected during $K=30$ epochs, and have $N=245520$ samples.

Once the state-of-the-art CPD-GEVD method and the SECSI method have previously shown the best results and have similar processing steps, we, additionally, performed 2000 MC simulations considering one NLOS and two NLOS. Thus, in Figure 3.14 the state-of-theart techniques are compared with the proposed SECSI based solution when having $L_{d}=3$ impinging signals. Note that the HOSVD+FBA+ESPS shows an almost constant error of approximately $0.28 \mathrm{~m}$ at $\Delta \tau=0.5 T_{c}$. Additionally, observe that the the state-of-the-art, DoA/KRF, and the proposed HOSVD SECSI solution achieve an overall error of approximately $0.082 \mathrm{~m}$. However, when having two NLOS the proposed HOSVD SECSI shows a larger error when $\Delta \tau<0.1 T_{c}$ and the CPD-GEVD have a larger error when $\Delta \tau<0.2 T_{c}$. This larger error is due to the highly correlated NLOS signals when $\Delta \tau<0.2 T_{c}$ which results in a rank deficient received tensor. This issue may be solved by properly estimating the model order before performing the CPD-GEVD. Therefore, we refer to [24, 25, 26]. Still, we note that the proposed HOSVD SECSI have a better performance when under highly correlated LOS and NLOS than the state-of-the-art CPD-GEVD. 


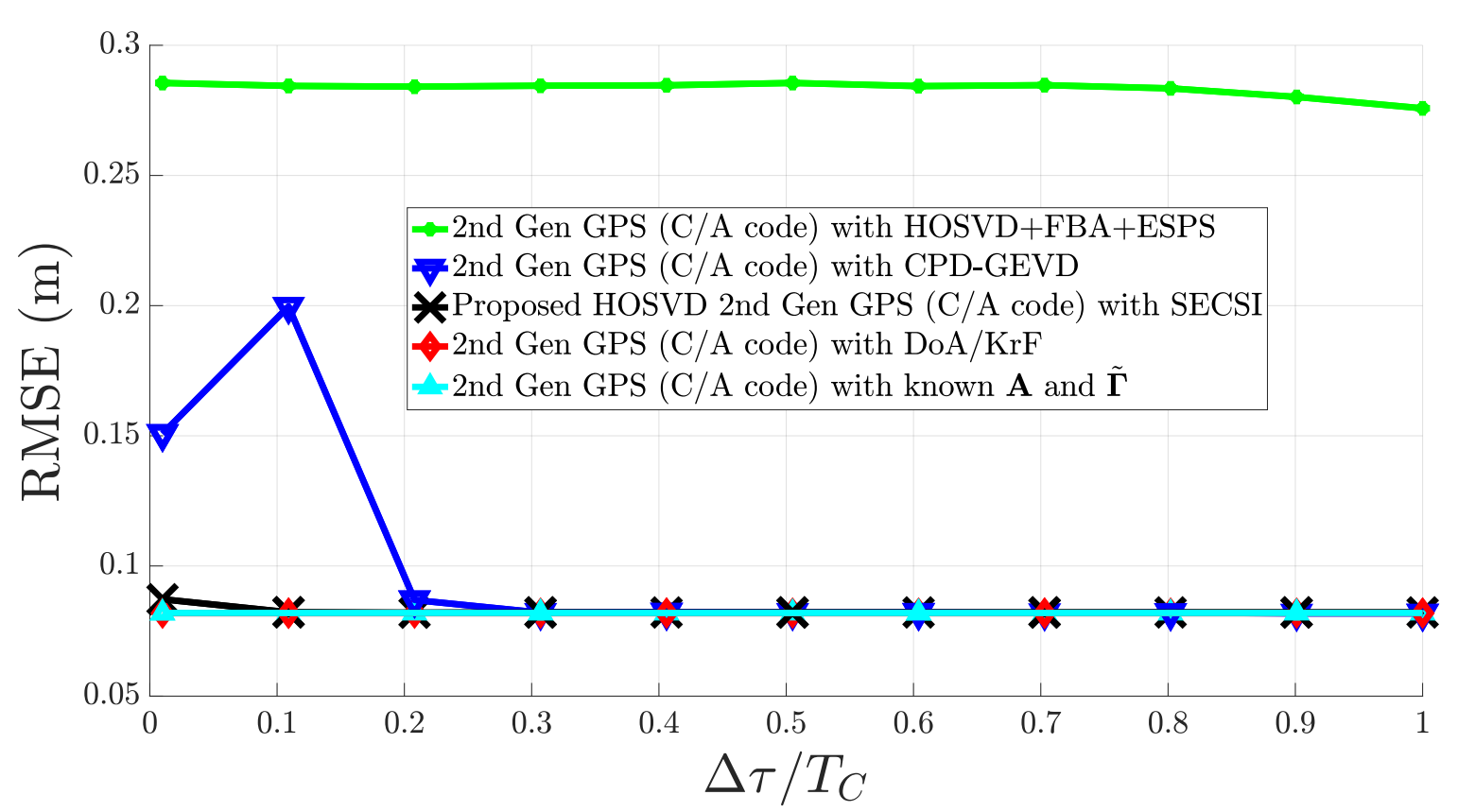

Figure 3.14: State-of-the-art techniques and proposed SECSI method simulation with $N=$ 245520 samples and $L_{d}=3$. In both cases code samples are collected during $K=30$ epochs with $M=8$ antenna.

\subsubsection{Third Generation GPS Time-Delay Estimation}

In this section we present simulation results for the state-of-the-art second generation GPS and the proposed third generation GPS using the proposed HOOI SECSI based timedelay estimation method. In this scenario both state-of-the-art techniques and SECSI method suppose an antenna array with $L_{d}=2$ impinging signals. Additionally, a scenario with $L_{d}=3$ impinging signals is considered. Once the SECSI method performs various matrix diagonalization, this method generates six factor estimates for our 3-way model. Similarly to the SECSI method, the CPD-GEVD can be modified to generate six factor estimates for our model. Lastly, we show that when using the the third generation GPS both state-of-the-art tensor based methods and the proposed SECSI method outperform the second generation GPS.

Similarly to the SECSI decomposition, the CPD-GEVD can produce six different factor estimates for different combinations of the GEVD step. Additionally to the proposed SECSI method, we performed simulations using the HOOI low-rank approximation instead of the HOSVD low-rank approximation. Therefore, Figure 3.15 shows the result for the state-ofthe-art CPD-GEVD based time-delay estimation, which displays an error of about 0.075 m. Still, in Figure 3.15 we show that the first factor estimate from the HOSVD SECSI methods match the state-of-the-art CPD-GEVD performance by having an error of about $0.75 \mathrm{~m}$. However, note that the HOOI SECSI displays a worst performance when the LOS and NLOS signal are most correlated. Still, by comparing with Figure 3.7 we notice that the third generation GPS outperforms the second generation GPS when $\Delta \tau<0.2 T_{c}$ by 
matching the ideal case.

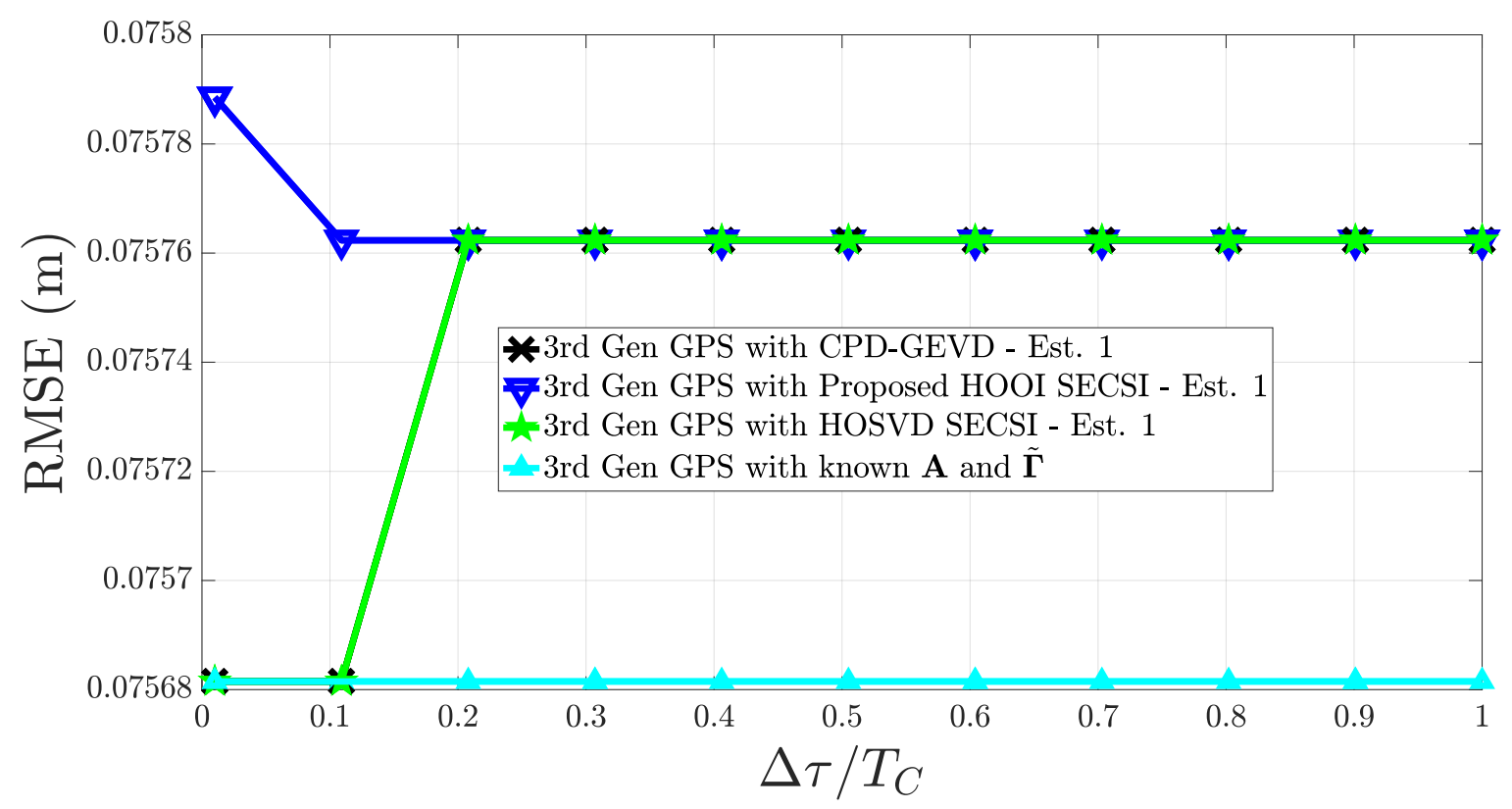

Figure 3.15: State-of-the-art CPD-GEVD, HOSVD SECSI, HOOI SECSI, and proposed SECSI method simulations using the first factor estimate with $M=8$ antennas and $L_{d}=2$ impinging signal. In both cases code samples are collected during $K=30$ epochs, and have $N=245520$ samples.

In Figure 3.16 we use the second estimate from both CPD-GEVD, HOOI SECSI, and HOSVD SECSI methods. Note that while the CPD-GEVD shows a large error when LOS and NLOS are highly correlated the SECSI, and HOOI SECSI perform the time-delay estimation with an overall error of about $0.075 \mathrm{~m}$ 


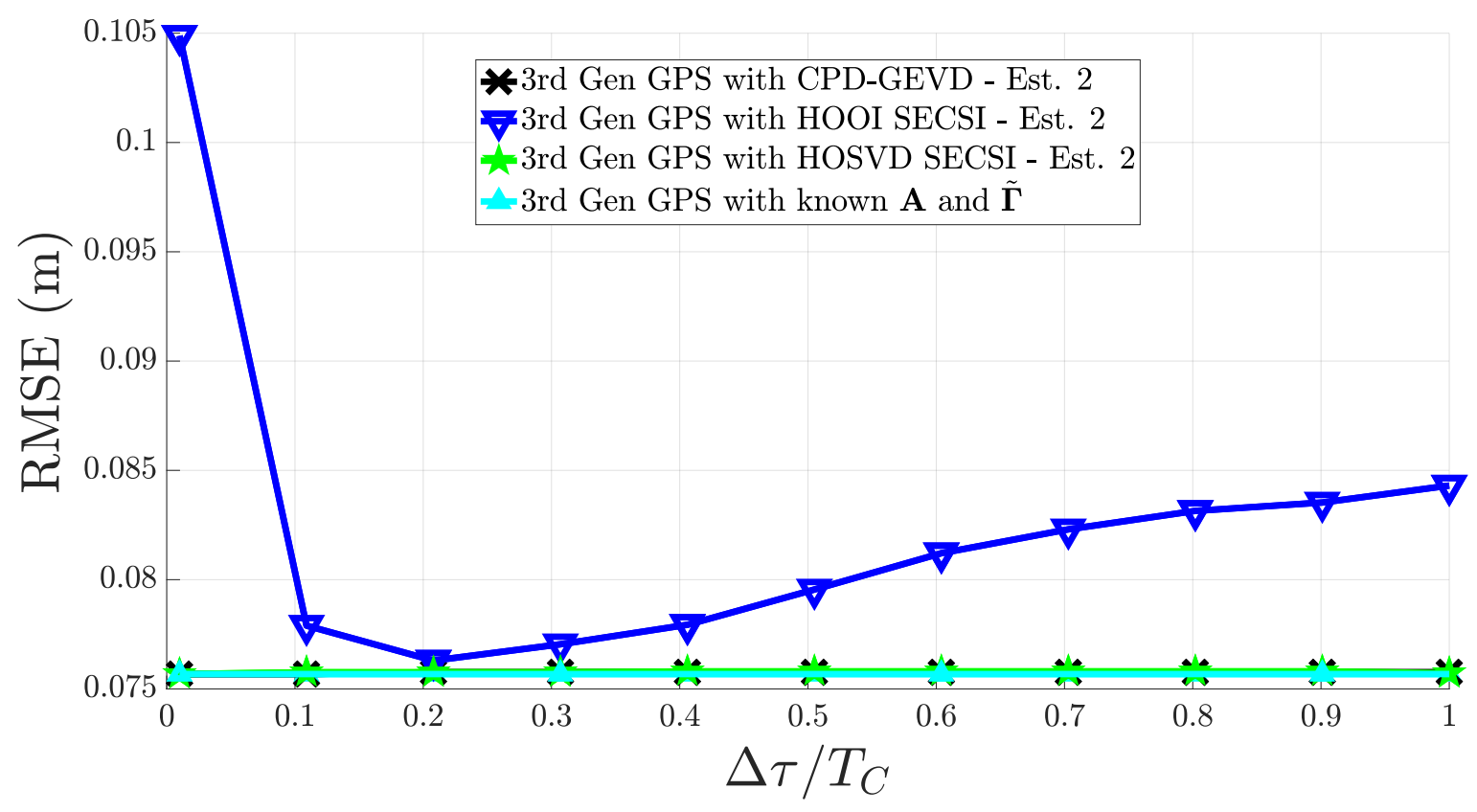

Figure 3.16: State-of-the-art CPD-GEVD, HOSVD SECSI, HOOI SECSI, and proposed SECSI method simulations using the second factor estimate with $M=8$ antennas and $L_{d}=2$ impinging signal. In both cases code samples are collected during $K=30$ epochs, and have $N=245520$ samples.

In Figure 3.17 we use the third estimate from both CPD-GEVD, HOOI SECSI, and HOSVD SECSI methods. Note that the third estimate displays the same result as the first estimated by having and overall error of about $0.075 \mathrm{~m}$ for both state-of-the-art CPD-GEVD and HOSVD SECSI and HOOI SECSI.

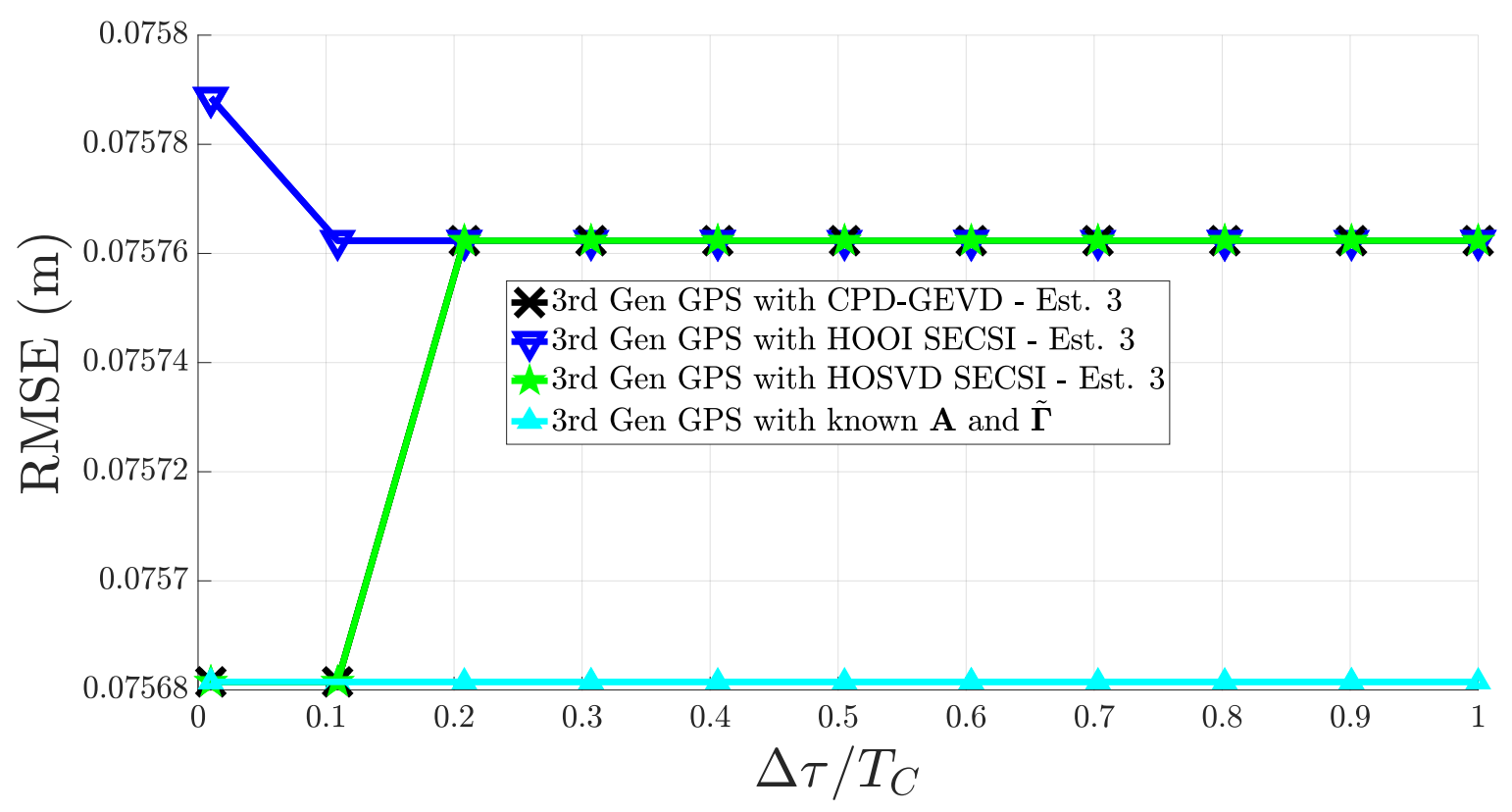

Figure 3.17: State-of-the-art CPD-GEVD, HOSVD SECSI, HOOI SECSI, and proposed SECSI method simulations using the third factor estimate with $M=8$ antennas and $L_{d}=2$ impinging signal. In both cases code samples are collected during $K=30$ epochs, and have $N=245520$ samples. 
Figure 3.18 displays the simulation results when using the fourth factor estimate from CPD-GEVD, HOOI SECSI, and HOSVD SECSI. Note that both HOSVD SECSI and HOOI SECSI have and unstable time-delay estimation while the CPD-GEVD an stable performance and successfully estimates the time-delay by showing an error of about $0.075 \mathrm{~m}$.

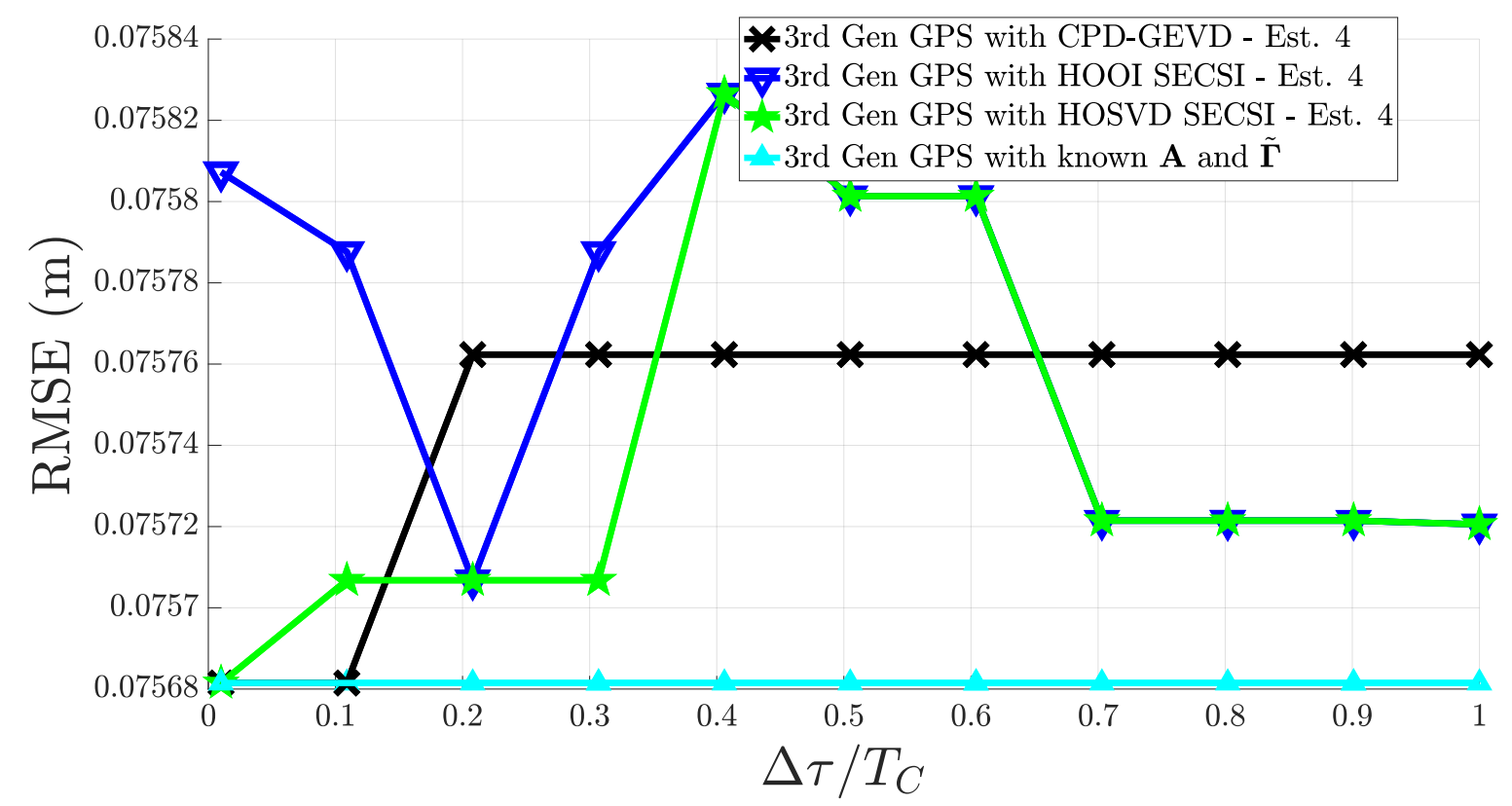

Figure 3.18: State-of-the-art CPD-GEVD, HOSVD SECSI, HOOI SECSI, and proposed SECSI method simulations using the fourth factor estimate with $M=8$ antennas and $L_{d}=2$ impinging signal. In both cases code samples are collected during $K=30$ epochs, and have $N=245520$ samples.

Figure 3.19 use the fifth factor estimate. Observe that similarly to the second generation the CPD-GEVD completely fails to estimate the time-delay by displaying a linear error. Still, the HOSVD SECSI successfully estimates the time-delay by having an approximate error of about $0.075 \mathrm{~m}$ while the HOOI SECSI shows a peak error of about $0.1 \mathrm{~m}$. 


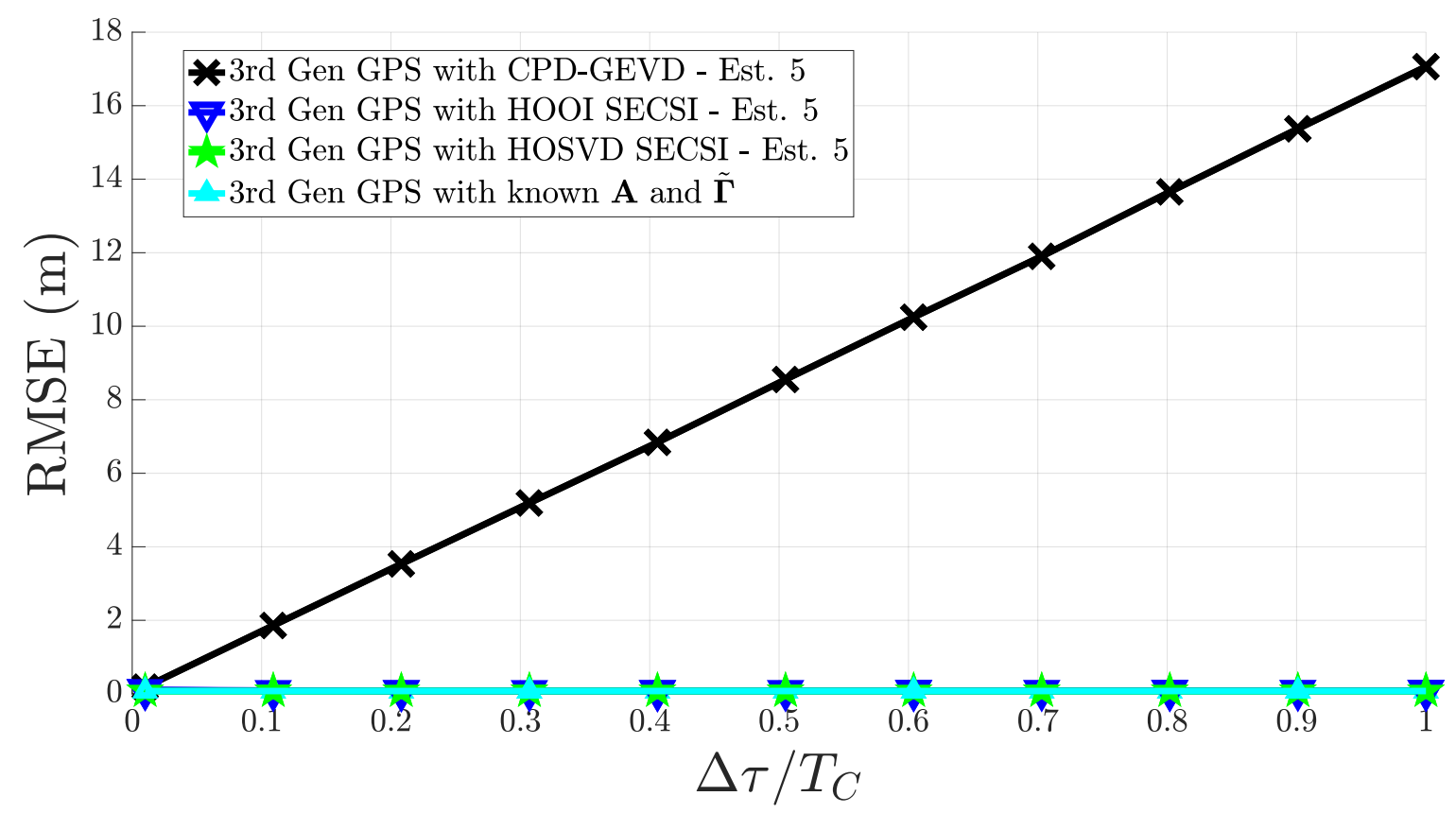

Figure 3.19: State-of-the-art CPD-GEVD, HOSVD SECSI, HOOI SECSI, and proposed SECSI method simulations using the fifth factor estimate with $M=8$ antennas and $L_{d}=2$ impinging signal. In both cases code samples are collected during $K=30$ epochs, and have $N=245520$ samples.

Lastly, Figure 3.20 use the sixth factor estimate. Observe that, again, the CPD-GEVD shows a linear error and fails to estimate the time-delay. However, both HOOI SECSI, and HOSVD SECSI methods present similar performance by having an approximate error of about $0.075 \mathrm{~m}$ 


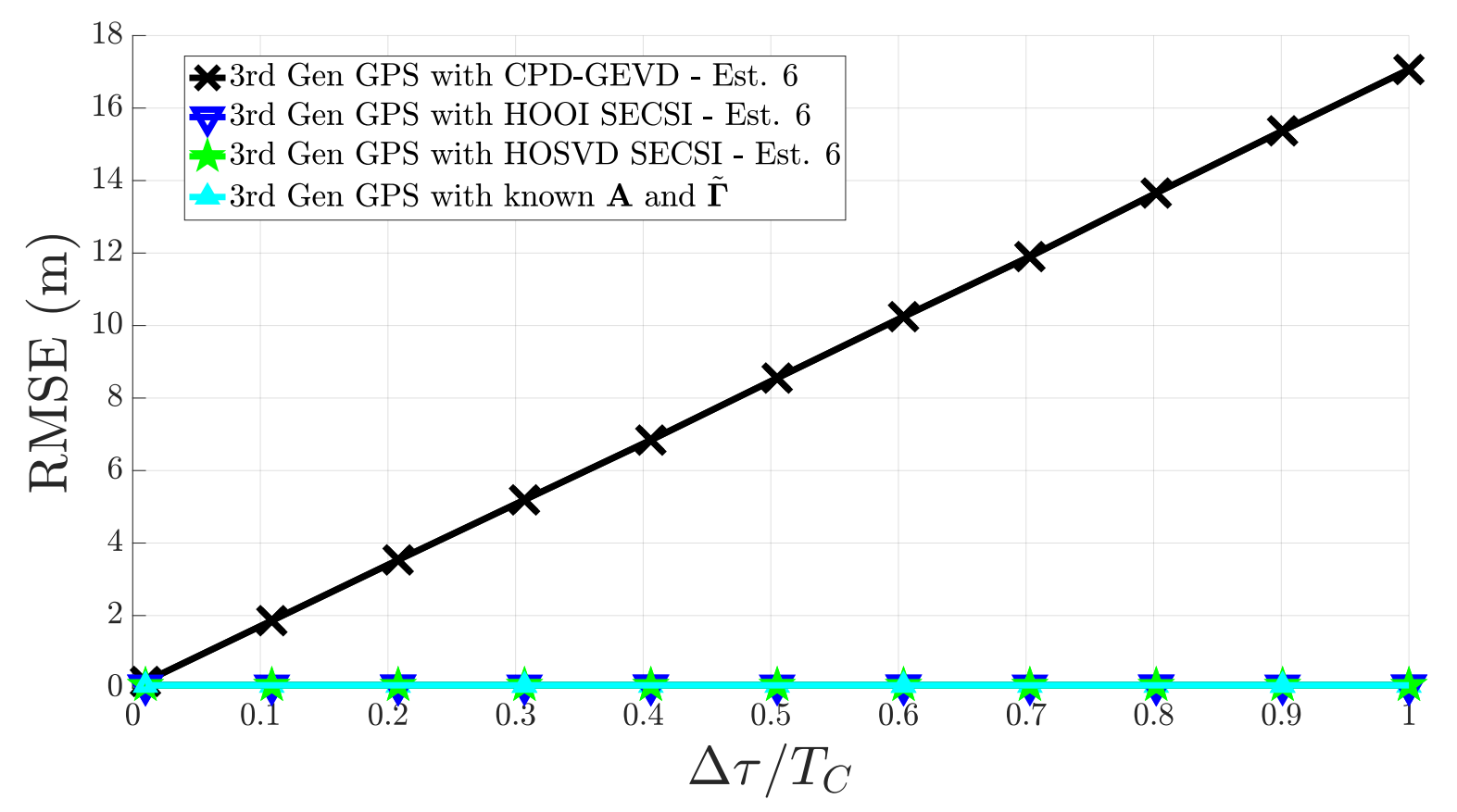

Figure 3.20: State-of-the-art CPD-GEVD, HOSVD SECSI, HOOI SECSI, and proposed SECSI method simulations using the sixth factor estimate with $M=8$ antennas and $L_{d}=2$ impinging signal. In both cases code samples are collected during $K=30$ epochs, and have $N=245520$ samples.

Once we show from Figures 3.15 through 3.20 that the CPD-GEVD presents the best results when using the first and third factor estimates, we decide to keep the state-of-the-art CPD-GEVD decomposition by using the first factor estimate. Once we show that the first and third estimates of the SECSI methods display the best performance and the process is similar to the state-of-the-art CPD-GEVD, we decided to pick the first factor estimate from the SECSI in order to perform time-delay estimation. Therefore, in Figure 3.21 the stateof-the-art techniques are compared with the proposed SECSI based solution. Both state-ofthe-art methods and the proposed SECSI method are equipped with an antenna array and receiving $L_{d}=2$ signals. Note that the state-of-the-art HOSVD+FBA+ESPS presents the worst result with a peak error of about $0.12 \mathrm{~m}$ at $\Delta \tau=0.3 T_{c}$. The state-of-the-art DoA/KRF and CPD-GEVD techniques and the proposed SECSI technique considerably outperform the state-of-the-art HOSVD+FBA+ESPS by showing the best results with an approximately error of around $0.075 \mathrm{~m}$. 


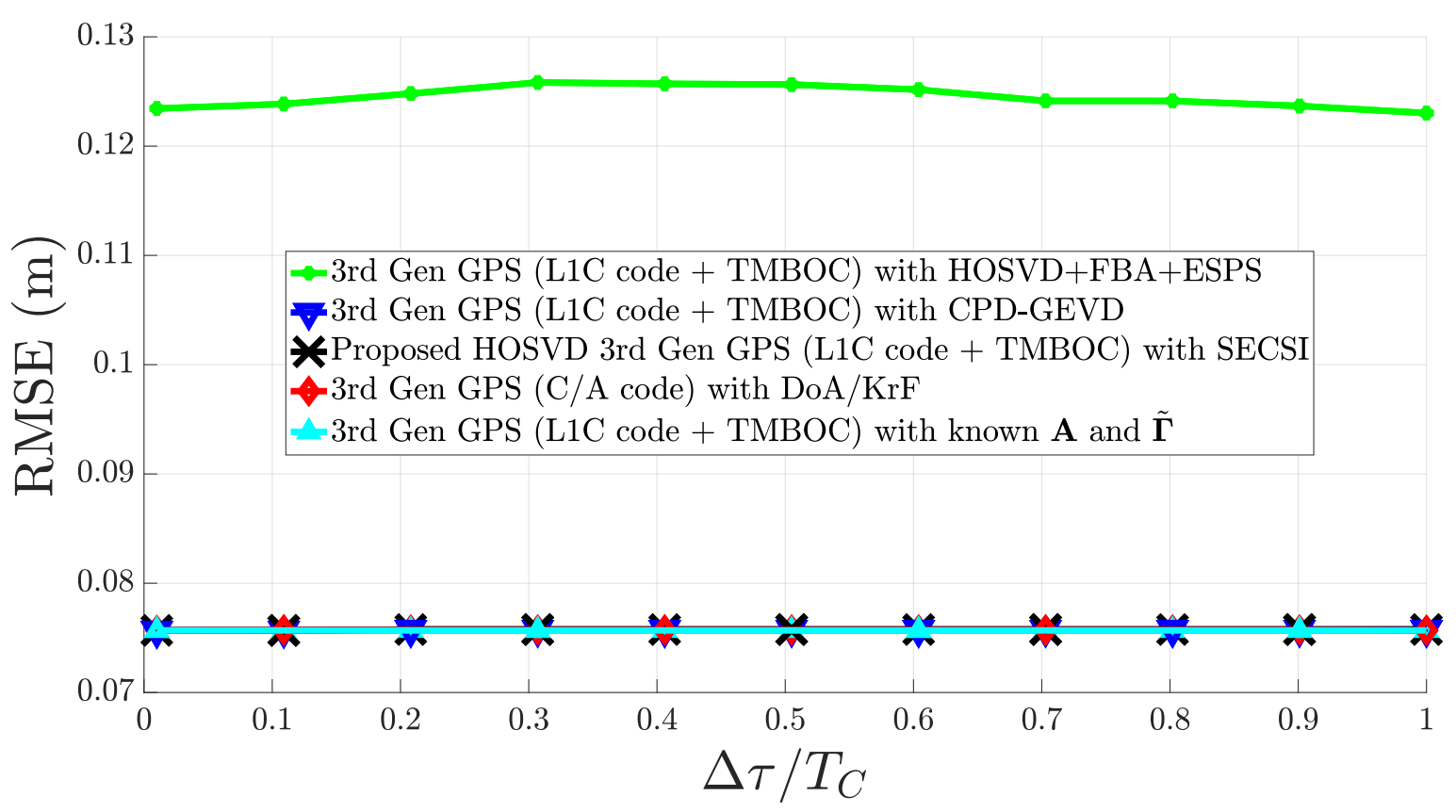

Figure 3.21: State-of-the-art techniques and proposed SECSI method simulation with $N=$ 245520 samples and $L_{d}=2$. In both cases code samples are collected during $K=30$ epochs with $M=8$ antenna.

In Figure 3.22 we have an antenna array that now have $L_{d}=3$ impinging signals and once the state-of-the-art CPD-GEVD method and the proposed SECSI method have similar solutions, the LOS component is obtained using the state-of-the-art CPD-GEVD and the first factor estimate obtained from the proposed HOSVD SECSI solution. Note that the HOSVD+FBA+ESPS shows an almost constant error of approximately $0.13 \mathrm{~m}$ which is similar to the error acquired when $L_{d}=2$. Note that the the state-of-the-art, DoA/KRF, and the proposed HOSVD SECSI solution achieve an overall error of approximately $0.078 \mathrm{~m}$ when $\Delta \tau>0.1 T_{c}$. However, when having two NLOS the proposed HOSVD SECSI shows a larger error when $\Delta \tau<0.1 T_{c}$ and the CPD-GEVD have a larger error when $\Delta \tau<0.2 T_{c}$. This larger error is due to the highly correlated NLOS signals when $\Delta \tau<0.2 T_{c}$ which results in a rank deficient received tensor. Still, we note that the proposed HOSVD SECSI have, again, a better performance when under highly correlated LOS and NLOS than the state-of-the-art CPD-GEVD. Furthermore, we observe that the third generation considerably outperforms the second generation once it has a lower time-delay error and shows a better performance under highly correlated noise when $L_{d}=3$. The better performance can be seen when comparing Figure 3.14 and Figure 3.22 and notice that the state-of-the-art CPDGEVD peak error at $\Delta \tau<0.1 T_{c}$ goes from approximately $0.2 \mathrm{~m}$ to about $0.08 \mathrm{~m}$. The better performance of the third generation GPS is a attributed to the TMBOC modulation once it introduces better performance in multipath scenarios and provide a better spectral separation of the signal. 


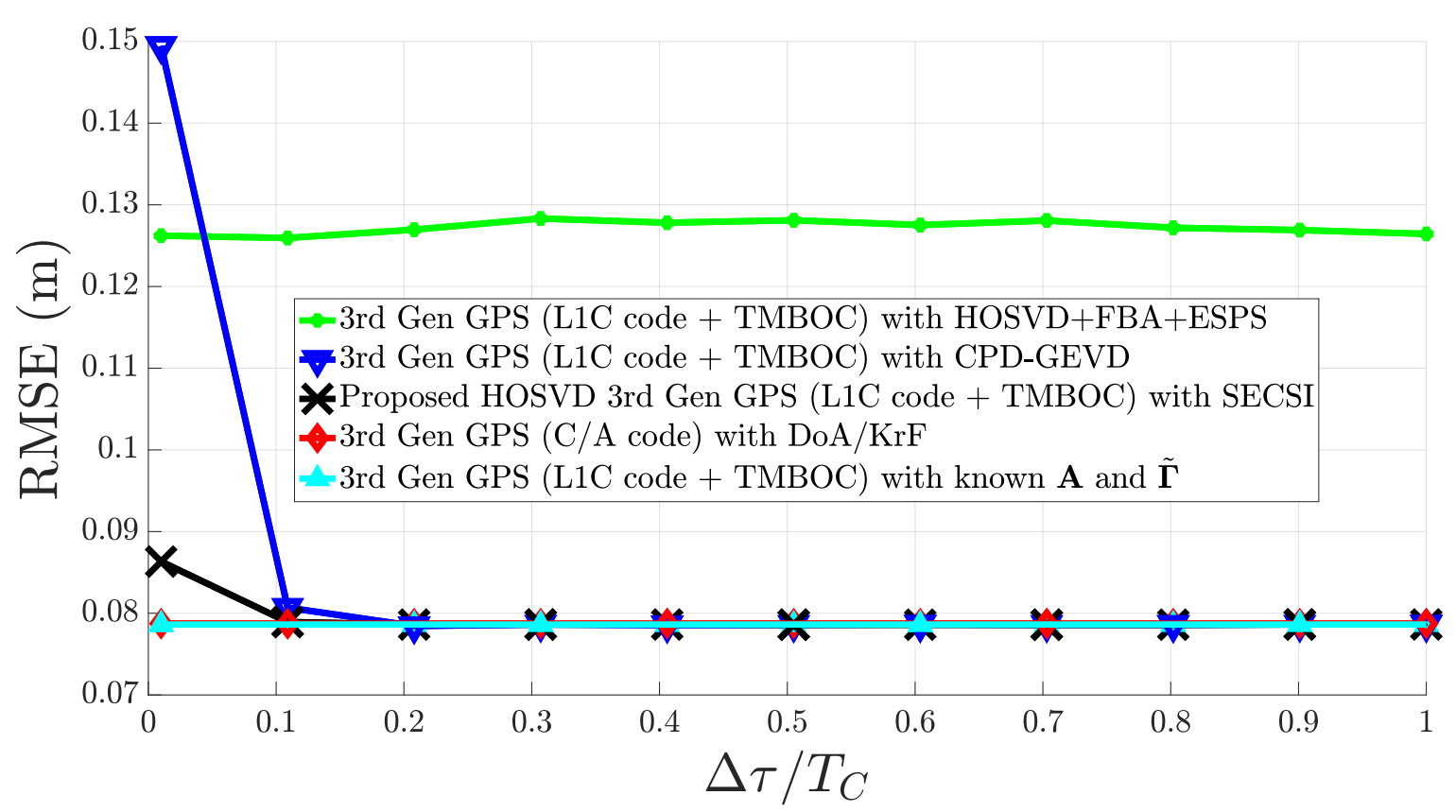

Figure 3.22: State-of-the-art techniques and proposed SECSI method simulation with $N=$ 245520 samples and $L_{d}=3$. In both cases code samples are collected during $K=30$ epochs with $M=8$ antenna.

Additionally, in Figure 3.23 and Figure 3.24 we compare both proposed second and third generation GPS SECSI based time-delay estimation. Furthermore, we compare the HOSVD SECSI based method against the HOOI SECSI based method when considering the first scenario with $L_{d}=2$. The LOS component is obtained using the first factor estimate obtained from the proposed SECSI solution for both HOSVD SECSI and HOOI SECSI based methods. Note that the HOOI SECSI and HOSVD based solution achieves a maximum error of about $0.079 \mathrm{~m}$ for the second generation GPS and a maximum error of about $0.75 \mathrm{~m}$ for the third generation GPS. We can note that the HOSVD SECSI slightly outperforms the HOOI SECSI when LOS and NLOS are strongly correlated. 


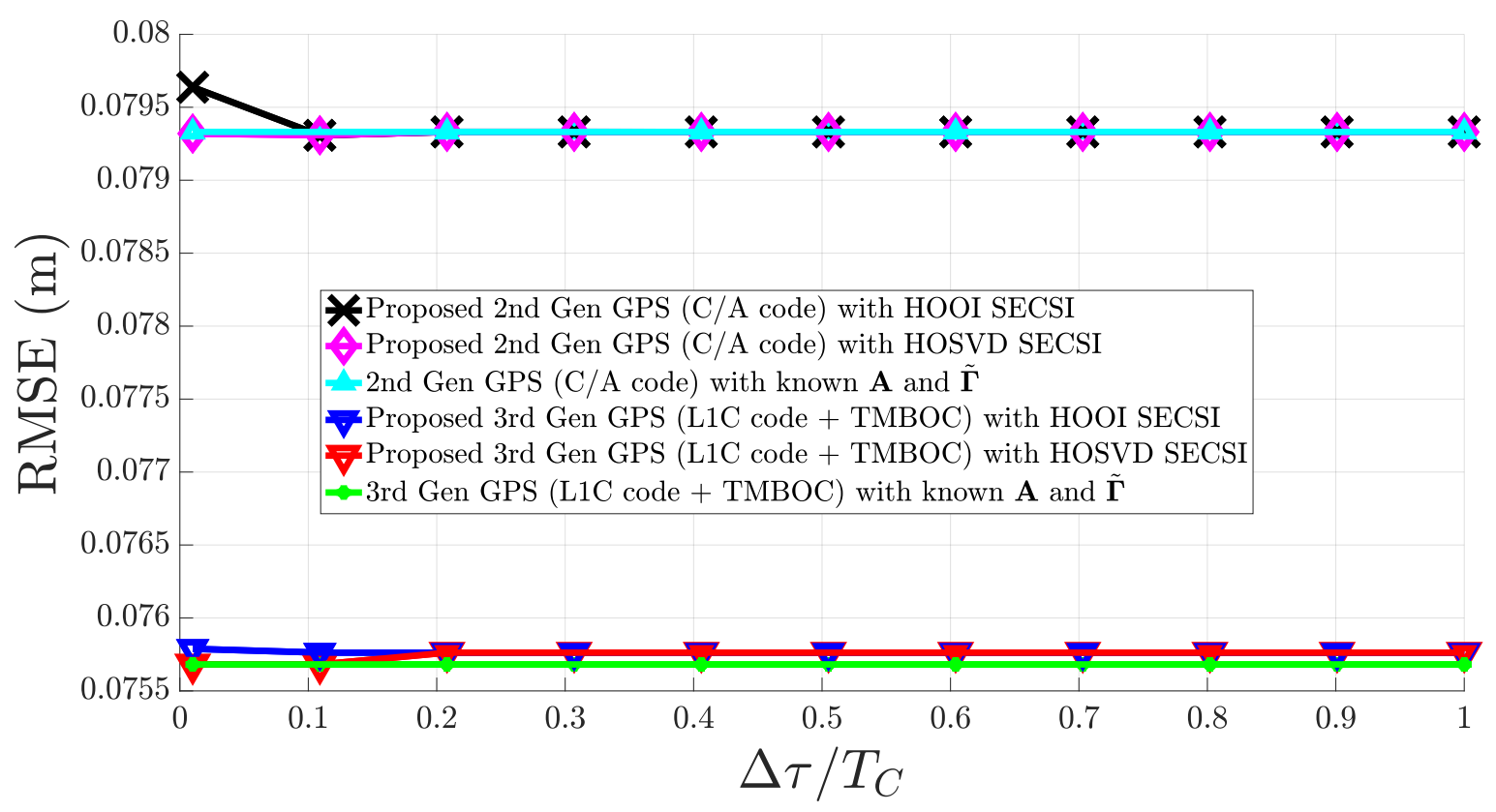

Figure 3.23: Proposed SECSI simulation with $L_{d}=2$ signals both 2 nd and 3rd with $N=$ 245520 samples. In both cases code samples are collected during $K=30$ epochs with $M=8$ antenna.

In Figure 3.24 we present the simulation results for the second scenario with $L_{d}=3$. The LOS component is obtained using the first factor estimate obtained from the proposed SECSI solution for both HOOI SECSI and HOSVD SECSI based methods. The proposed second generation GPS based solution successfully perform the time-delay estimation by achieving an error of approximately $0.082 \mathrm{~m}$ when $\Delta \tau<0.1 T_{c}$. The third generation GPS with the proposed HOSVD SECSI and the HOOI SECSI method achieves a peak error of about $0.09 \mathrm{~m}$ when $\Delta \tau<0.1 T_{c}$ once the LOS and NLOS are highly correlated. Therefore, we show that the proposed HOSVD SECSI and HOOI SECSI based time-delay estimation have similar performance when using both second and third generation GPS. 


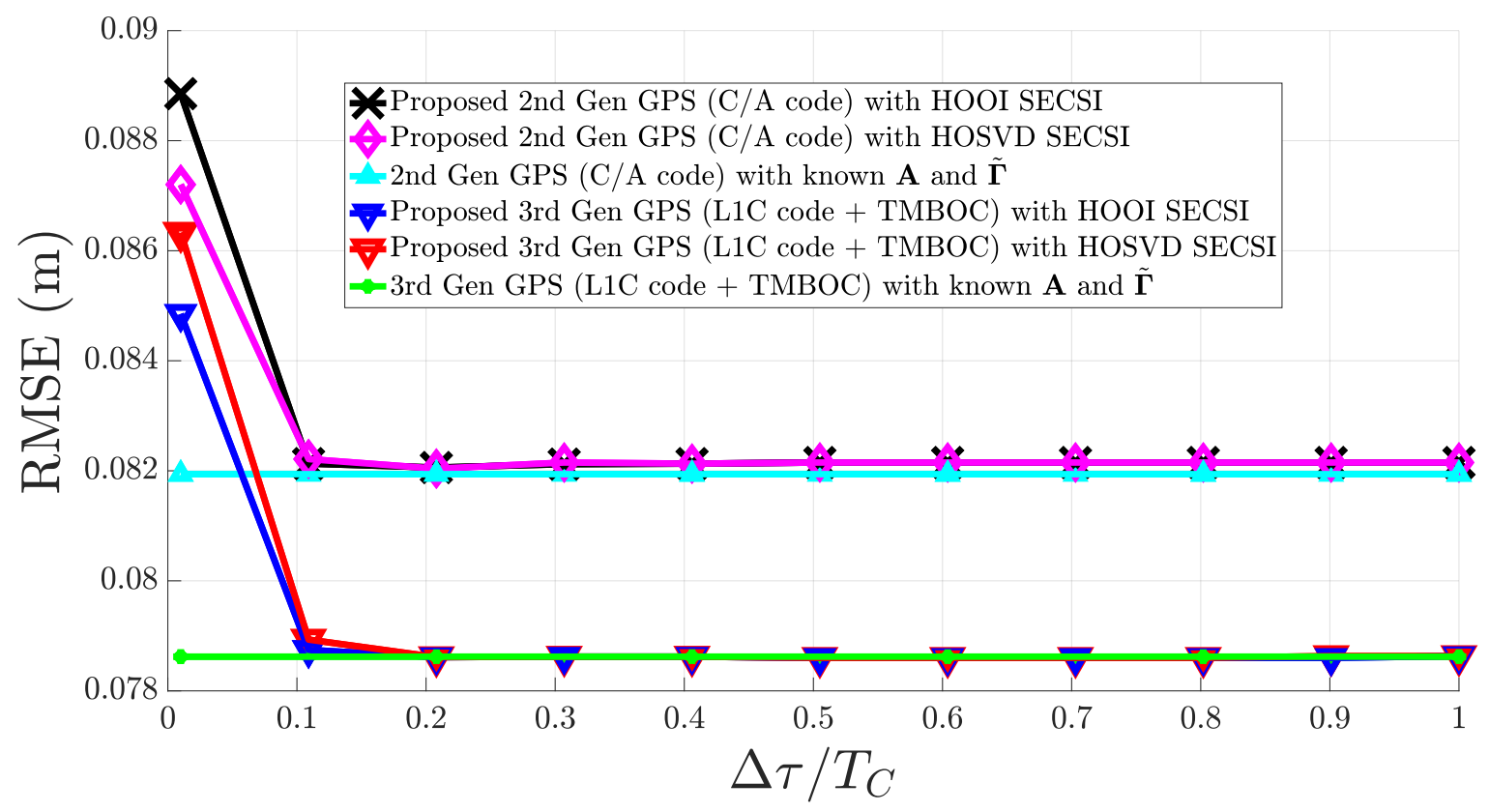

Figure 3.24: Proposed SECSI simulation with $L_{d}=3$ signals both 2 nd and 3rd with $N=$ 245520 samples. In both cases code samples are collected during $K=30$ epochs with $M=8$ antenna.

\subsubsection{Antenna array with errors}

In this section we present simulation results for the state-of-the-art second generation GPS and the proposed third generation GPS using the proposed HOSVD SECSI and HOOI SECSI based time-delay estimation method. In this scenario both state-of-the-art techniques and SECSI method suppose an antenna array with error with $L_{d}=2$ and $L_{d}=3$ impinging signals and a fixed relative delay $\Delta \tau$ at $0.5 T_{c}$. Once we decided to peak the first factor estimate of CPD-GEVD and SECSI methods, in this section we only present the simulation results to these case. Lastly, we show that when using the the third generation GPS both state-of-the-art tensor based methods and the proposed SECSI method outperform the second generation GPS.

In Figure 3.25 we show the results for the second generation when considering the antenna array with errors and $L_{d}=2$ impinging signals. We show that the state-of-the-art HOSVD+FBA+ESPS and DoA/KRF are sensitive to imperfections added to the antenna array with an error of about $0.99 \mathrm{~m}$ at $p=10^{-1}$ and $3.7 \mathrm{~m}$ at $p=10^{-1.5}$, respectively. However, the state-of-the-art CPD-GEVD and the proposed HOSVD SECSI as well as the HOOI SECSI can maintain similar performance as when using a perfect array. 


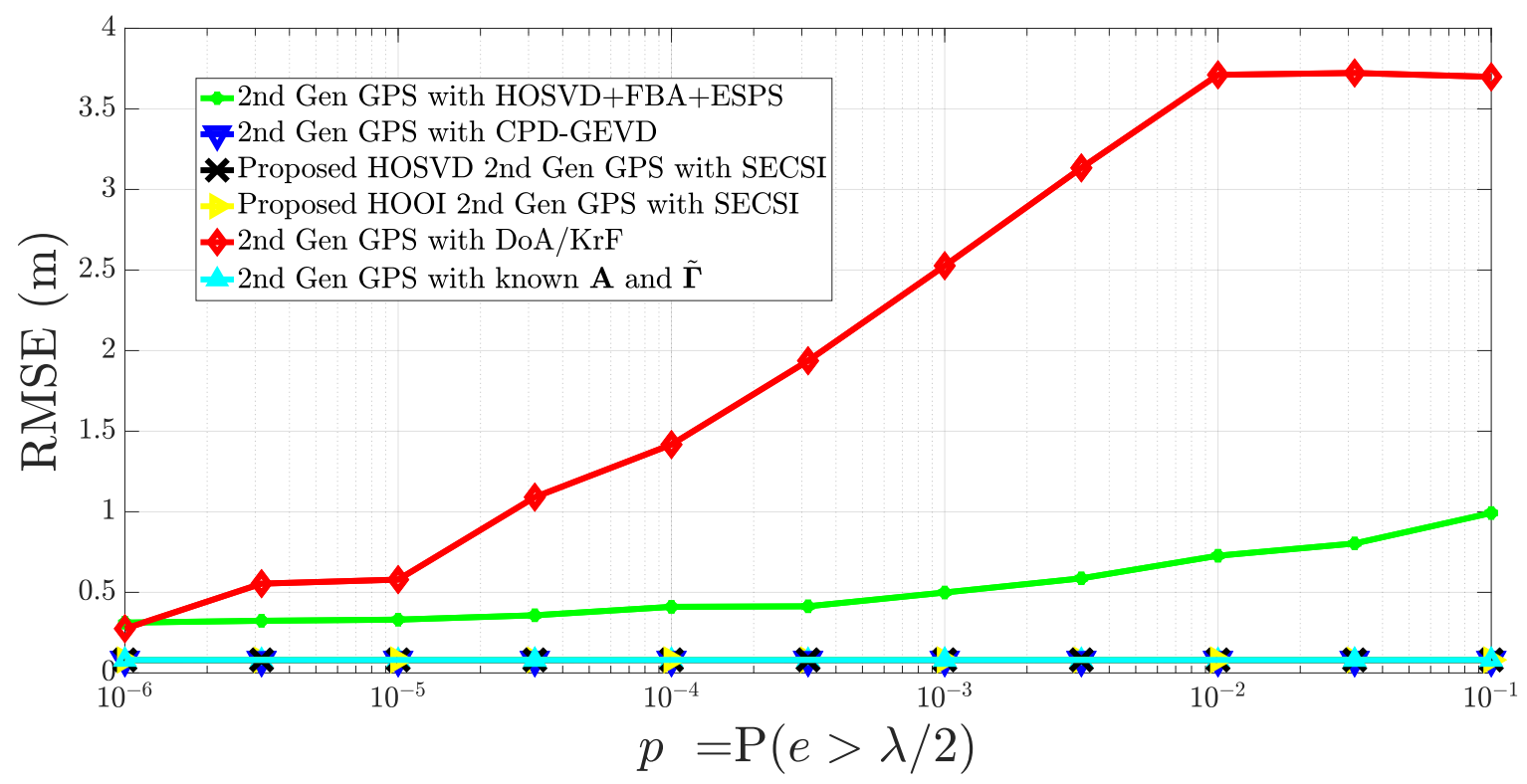

Figure 3.25: State-of-the-art techniques and Proposed SECSI simulation with $L_{d}=2$ signals for 2 nd generation with $N=245520$ samples. In both cases code samples are collected during $K=30$ epochs with $M=8$ antenna.

In Figure 3.26 we show the results for the third generation when considering the antenna array with errors and $L_{d}=2$ impinging signals. Again, even with the third generation the state-of-the-art HOSVD+FBA+ESPS and DoA/KRF are sensitive to imperfections added. Actually, the third generation GPS signal do not introduces a significant gain when compared with the results obtained when using the second generation GPS once it shows an error of about $0.92 \mathrm{~m}$ at $p=10^{-1}$ for HOSVD+FBA+ESPS and $3.69 \mathrm{~m}$ at $p=10^{-1.5}$ for DoA/KRF. Furthermore, the state-of-the-art CPD-GEVD and the proposed HOSVD SECSI and HOOI SECSI are able to perform the time-delay estimation and show similar performance to the scenario with a perfect array.

In Figure 3.27 we show the results for the second generation when considering the antenna array with errors and $L_{d}=3$ impinging signals. We show that by adding an extra NLOS component we have an increase in the time-delay estimation when using the state-ofthe-art HOSVD+FBA+ESPS and DoA/KRF. Moreover, the state-of-the-art CPD-GEVD and the proposed HOSVD SECSI and HOOI SECSI are robust against array imperfections and show similar results to the scenario with a perfect array.

In Figure 3.28 we show the results for the third generation when considering the antenna array with errors and $L_{d}=3$ impinging signals. We show that by adding an extra NLOS component we have an increase in the time-delay estimation when using the state-of-the-art HOSVD+FBA+ESPS and DoA/KRF. However, we can notice that the addition of the extra NLOS has a low impact in the time-delay estimation once the HOSVD+FBA+ESPS has an increase of about $0.1 \mathrm{~m}$ and the DoA/KRF maintain the same error. In addition, observe that the third generation GPS with $L_{d}=3$ displays a lower error than the second generation GPS with $L_{d}=2$. Furthermore, the state-of-the-art CPD-GEVD and the proposed HOSVD 


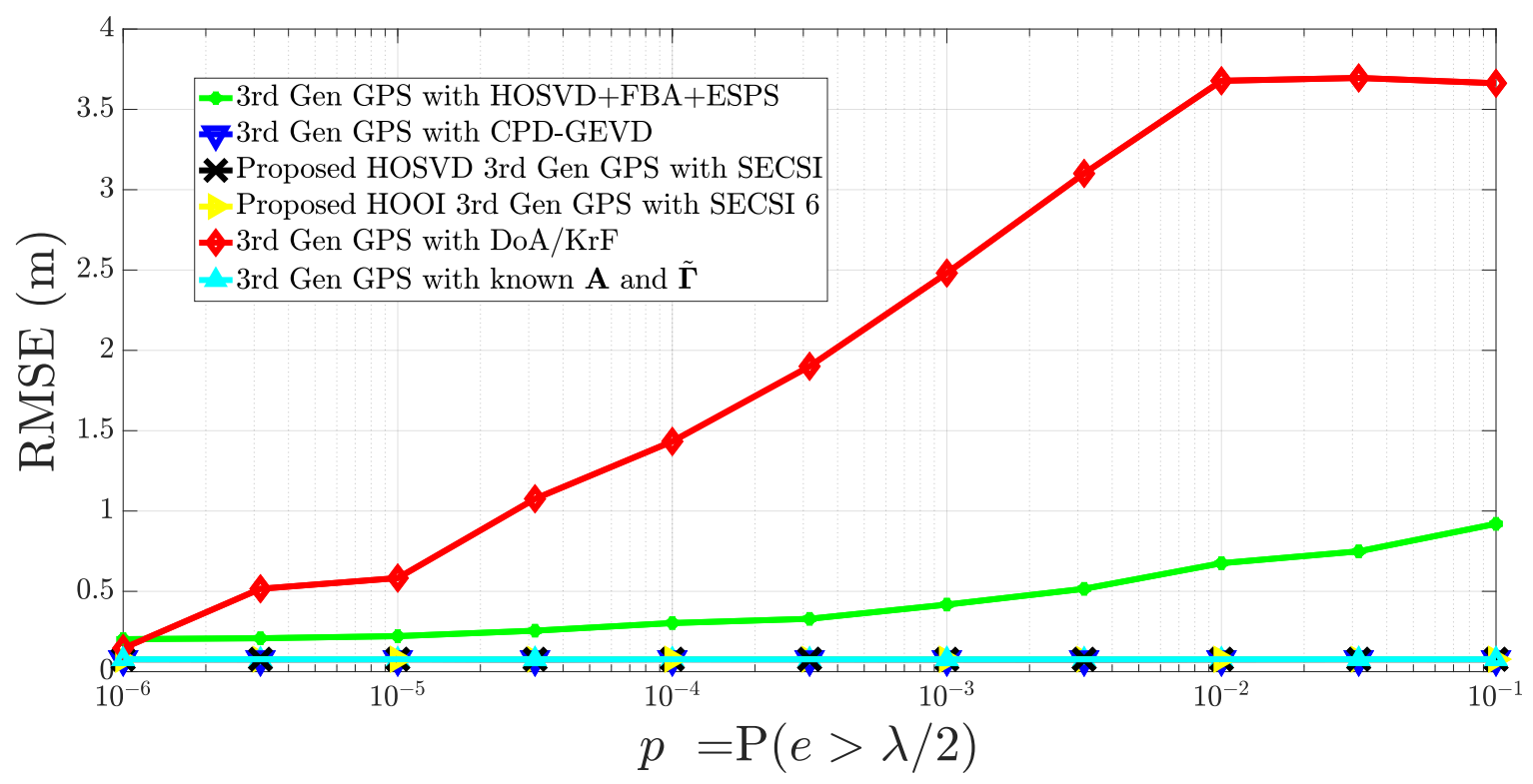

Figure 3.26: State-of-the-art techniques and Proposed SECSI simulation with $L_{d}=2$ signals for 3rd Gen with $N=245520$ samples. In both cases code samples are collected during $K=30$ epochs with $M=8$ antenna.

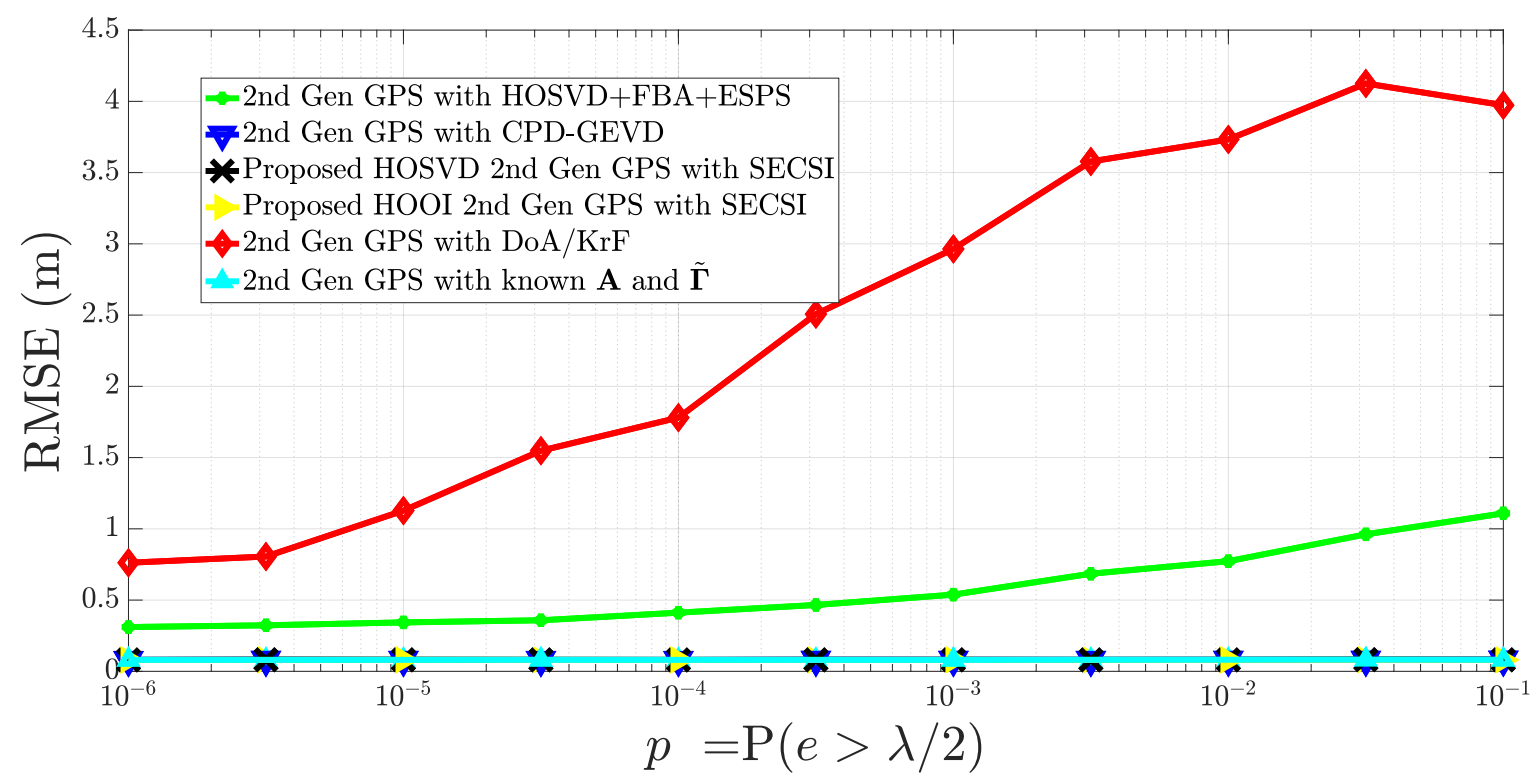

Figure 3.27: State-of-the-art techniques and Proposed SECSI simulation with $L_{d}=3$ signals for 2 nd Gen with $N=245520$ samples. In both cases code samples are collected during $K=30$ epochs with $M=8$ antenna. 


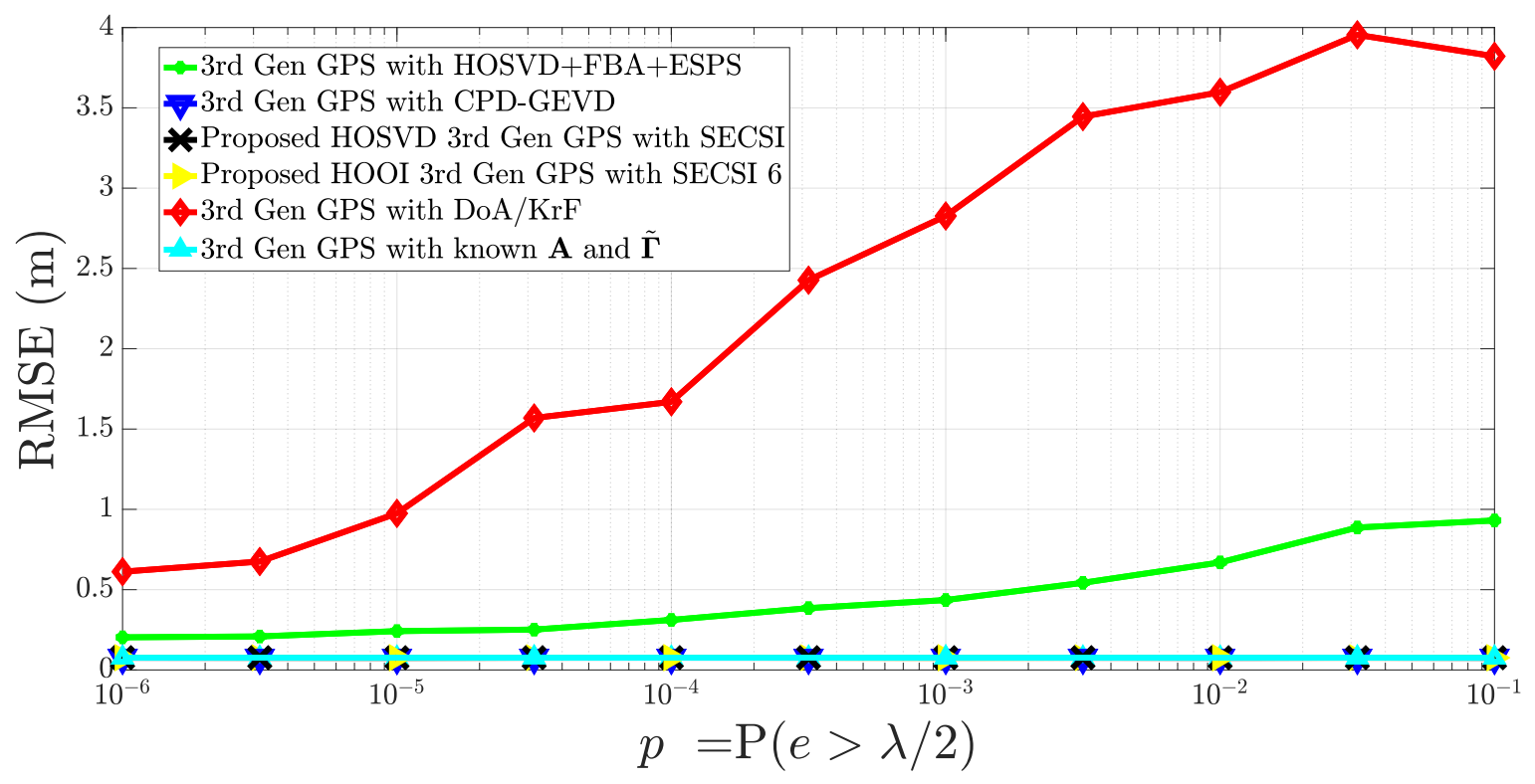

Figure 3.28: State-of-the-art techniques and Proposed SECSI simulation with $L_{d}=3$ signals for 3rd with $N=245520$ samples. In both cases code samples are collected during $K=30$ epochs with $M=8$ antenna.

SECSI and HOOI SECSI are robust against array imperfections and show similar results to the scenario with a perfect array.

\subsubsection{Received Signals with Varying DoA}

In this section we present simulation results for the state-of-the-art second generation GPS and the proposed third generation GPS using the proposed HOSVD SECSI based timedelay estimation method. We again suppose two scenarios using the state-of-the-art techniques and SECSI method. These two scenarios suppose an antenna array with $L_{d}=2$ and $L_{d}=3$ impinging signals and a fixed relative delay $\Delta \tau$ at $0.5 T_{c}$. When we have $L_{d}=2 \mathrm{im}$ pinging signals we define the first NLOS as having an angle of arrival $\phi_{\mathrm{NLOS}_{1}}=\phi_{\mathrm{LOS}}+\Delta \phi$ and when we have $L_{d}=3$ signal components we define a second NLOS with an angle of arrival $\phi_{\mathrm{NLOS}_{2}}=\phi_{\mathrm{NLOS}_{1}}+\Delta \phi$. Still, both scenarios suppose we draw a random angle from $-0.25 \mathrm{rad}$ to $0.25 \mathrm{rad}$ for LOS component and add eleven different angles of arrival to each NLOS. Therefore, each simulation will vary the NLOS angle of arrival based on the LOS angle of arrival. In this sense, we can evaluate the minimum DoA difference from LOS to NLOS. We again use the first factor estimate of CPD-GEVD and SECSI methods, in this section we only present the simulation results to these case.

In Figure 3.29 we show the results for the second generation when considering we receive $L_{d}=2$ impinging signals, where we use LOS DoA to compute the NLOS DoA. We show that the state-of-the-art DoA/KRF is sensitive to the angle of arrival. However, it stabilizes when we have $\Delta \phi=0.005 \times 0.25 \mathrm{rad}$. Moreover, the state-of-the-art CPD-GEVD and the proposed HOSVD SECSI have a higher error than DoA/KRF when we have a $\Delta \phi=0 \times 0.25$ rad, which in practice means we have only one signal being received. However, the state-of- 
the-art CPD-GEVD and the proposed SECSI outperforms the DoA/KRF method once these methods stabilizes at $\Delta \phi=0.0025 \times 0.25 \mathrm{rad}$.

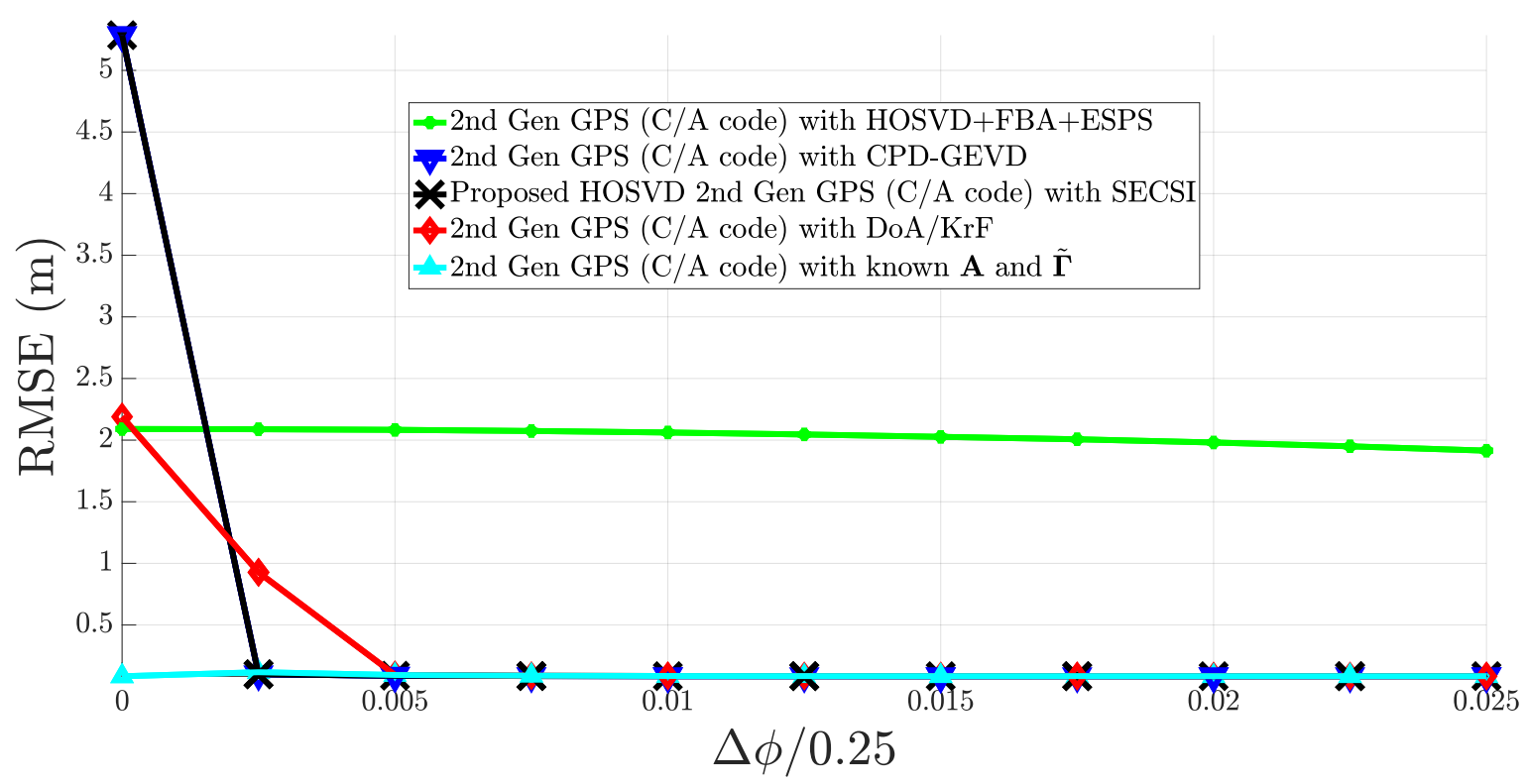

Figure 3.29: State-of-the-art techniques and Proposed SECSI simulation with $L_{d}=2$ signals for 2 nd Gen with $N=245520$ samples. In both cases code samples are collected during $K=30$ epochs with $M=8$ antenna.

In Figure 3.30 we show results for second generation when considering we have $L_{d}=3$ impinging signals, where we use LOS DoA to compute the NLOS DoA. We show that the state-of-the-art DoA/KRF is sensitive to the angle of arrival. However, it stabilizes when we have $\Delta \phi=0.015 \times 0.25 \mathrm{rad}$. Moreover, the state-of-the-art CPD-GEVD and the proposed HOSVD SECSI have a higher error than DoA/KRF when we have a $\Delta \phi=0 \times 0.25$ rad, which in practice means we have only one signal being received. However, the stateof-the-art CPD-GEVD and the proposed SECSI outperforms the DoA/KRF method once these methods stabilizes at $\Delta \phi=0.025 \times 0.25 \mathrm{rad}$. Still, we see that the proposed SECSI outperforms the CPD-GEVD technique when we have a DoA difference $\Delta \phi=0 \times 0.25$. 


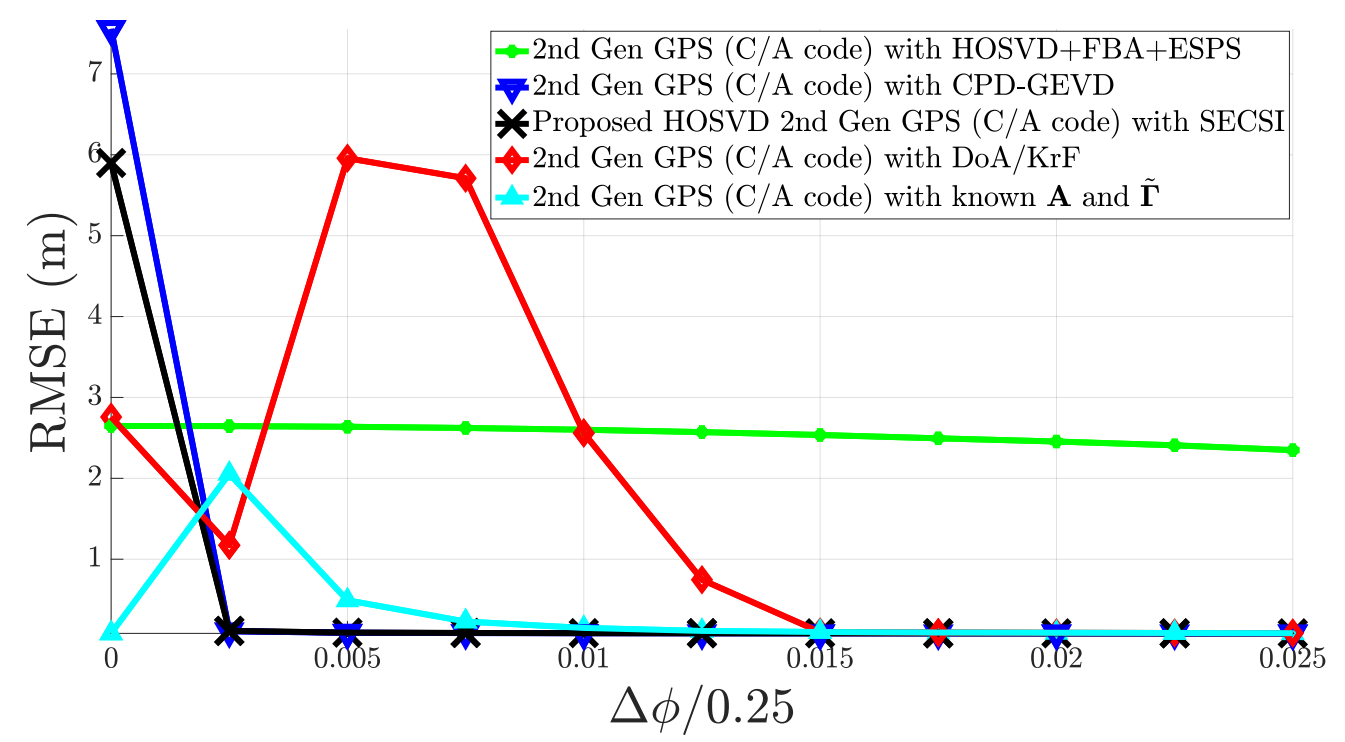

Figure 3.30: State-of-the-art techniques and Proposed SECSI simulation with $L_{d}=3$ signals for 2 nd Gen with $N=245520$ samples. In both cases code samples are collected during $K=30$ epochs with $M=8$ antenna.

In Figure 3.31 we show results for third generation when considering we have $L_{d}=2$ impinging signals. Again we show that the state-of-the-art DoA/KRF is sensitive to the angle of arrival. However, it stabilizes when we have $\Delta \phi=0.005 \times 0.25 \mathrm{rad}$. Moreover, the state-of-the-art CPD-GEVD and the proposed HOSVD SECSI have a higher error than DoA/KRF when we have a $\Delta \phi=0 \times 0.25 \mathrm{rad}$, which in practice means we have only one signal being received. Furthermore, observe that the third generation GPS slightly improves the time-delay estimation.

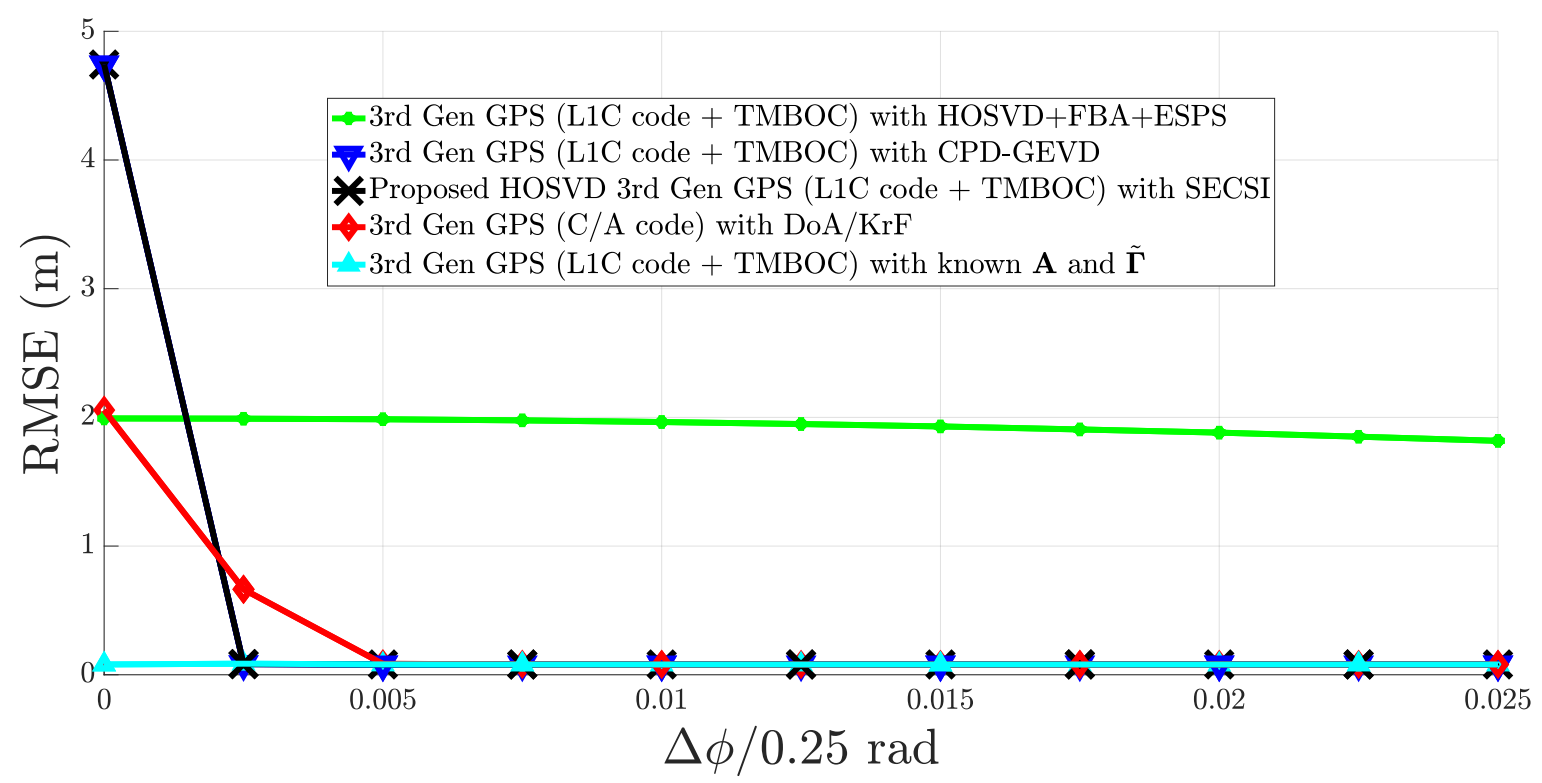

Figure 3.31: State-of-the-art techniques and Proposed SECSI simulation with $L_{d}=2$ signals for 2 nd Gen with $N=245520$ samples. In both cases code samples are collected during $K=30$ epochs with $M=8$ antenna. 
In Figure 3.32 we show results for third generation when considering we have $L_{d}=3$ impinging signals. Note that the state-of-the-art DoA/KRF is sensitive to the angle of arrival. However, it stabilizes when we have $\Delta \phi=0.015 \times 0.25 \mathrm{rad}$. Moreover, the state-of-the-art CPD-GEVD and the proposed HOSVD SECSI have a higher error than DoA/KRF when we have a $\Delta \phi=0 \times 0.25 \mathrm{rad}$, which in practice means we have only one signal being received. Therefore, observe that the proposed SECSI improves the time-delay estimation. Furthermore, note that the third generation GPS display a lower error than its second generation GPS counterpart.

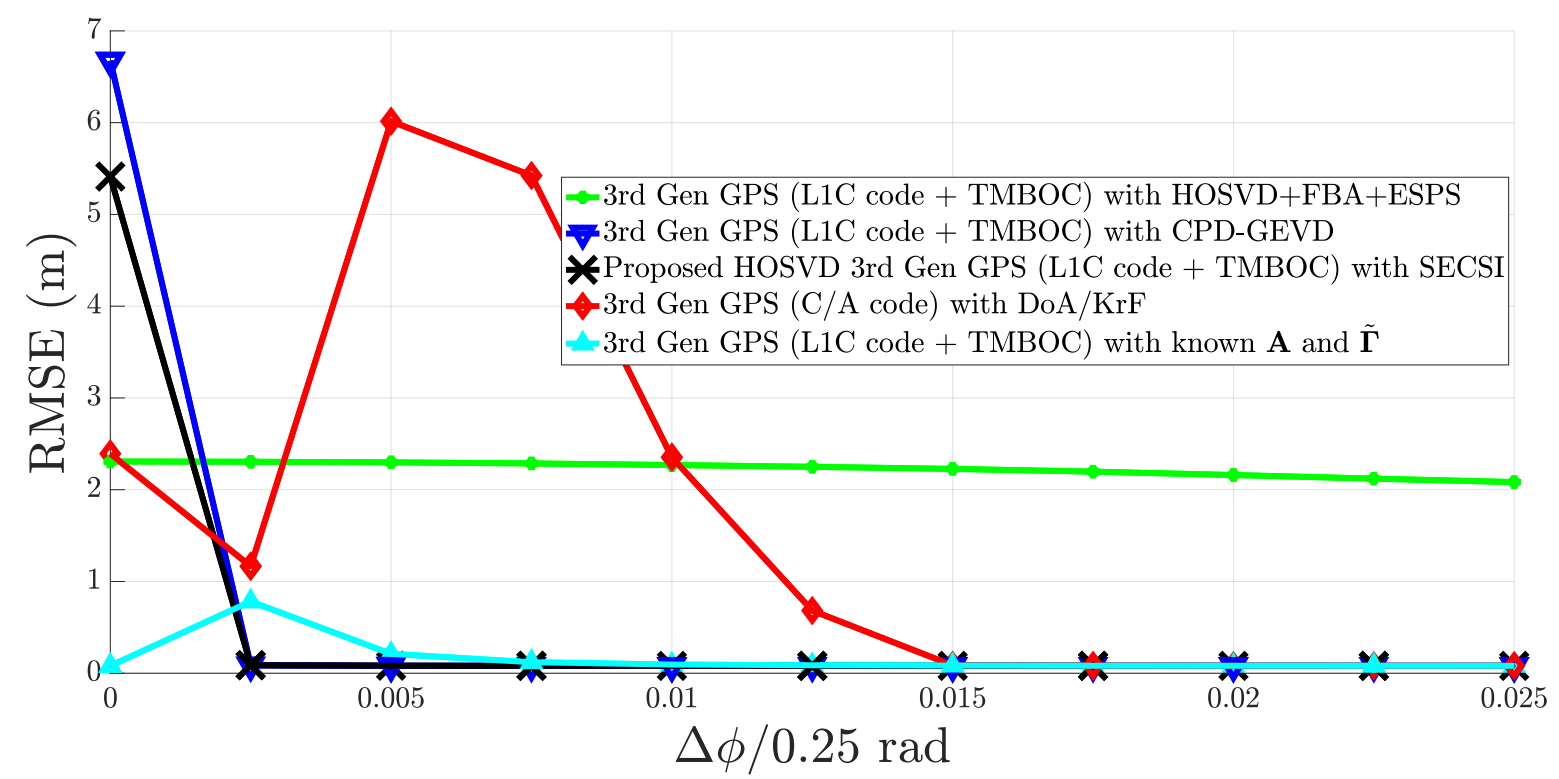

Figure 3.32: State-of-the-art techniques and Proposed SECSI simulation with $L_{d}=3$ signals for 2 nd Gen with $N=245520$ samples. In both cases code samples are collected during $K=30$ epochs with $M=8$ antenna. 


\section{Chapter 4}

\section{Conclusion}

Hereby we studied both state-of-the-art Higher Order Singular Value Decomposition (HOSVD), Canonical Polyadic Decomposition - Generalized Eigenvalue Decomposition (CPD-GEVD), and proposed to use the Semi-algebraic framework for approximate Canonical Polyadic decomposition via simultaneous matrix diagonalization (SECSI), which outperforms the current state-of-the-art-techniques for multipath mitigation and time-delay estimation. In addition, both state-of-the-art method and the proposed method showed compatibility with the C/A signals as well as with the L1C signaling combined with TMBOC.

Furthermore, we explored all possible factor estimates yielded by a SECSI Decomposition with Higher Order Singular Value Decomposition (HOSVD) low-rank approximation and Higher Order Orthogonal Iteration (HOOI) low-rank approximation by performing 2000 MC simulations using two different scenarios. The first scenario considered an antenna array receiving $L_{d}=2$ signals and showed that in this case the first and third factor estimated by the SECSI with HOSVD and HOOI can be used to perform time-delay estimation. Yet, we denote that SECSI with HOSVD yielded similar result to the state-of-the-art CPD-GEVD when combined with both second and third generation GPS. Still, we used the same scenario however we substituted the HOSVD low-rank approximation by the HOOI low-rank approximation technique combined with the SECSI decomposition. This time, we observed that when using the HOOI method we still have a similar performance with the state-of-theart CPD-GEVD. Additionally, we showed that the SECSI with HOSVD or HOOI have a better performance when the LOS and NLOS are strongly correlated.

Additionally to the first scenario, we performed $2000 \mathrm{MC}$ with an antenna array receiving $L_{d}=3$ signals from both second and third generation GPS. Again, we used the HOOI and HOSVD methods combined with the SECSI decomposition. In this scenario we showed that the second generation GPS has a similar performance when combined with the SECSI with HOSVD or HOOI method. Furthermore, we showed that the SECSI with HOSVD and HOOI yield a smaller error when combined with the third generation GPS. Furthermore, we showed that SECSI with HOSVD or HOOI have a better performance with with third generation GPS under strongly correlated LOS and NLOS signals. Additionally, we showed state-of-the-art 
CPD-GEVD combined with the third generation GPS considerably outperforms the second generation GPS when having strongly correlated signals.

Finally, we performed $2000 \mathrm{MC}$ considering an antenna array with imperfections and receiving $L_{d}=2$ and $L_{d}=3$ signals. Therefore, we show that the state-of-the-art CPD-GEVD and the proposed HOSVD SECSI and HOOI SECSI are robust against antenna array imperfections and have similar performance when compared with the simulations using a perfect array. Moreover, we show that the third generation GPS scheme with $L_{d}=3$ impinging signals has a better performance than the second generation GPS scheme with $L_{d}=2$.

\subsection{Future Works}

We have shown in our simulations that the CPD-GEVD has a poor performance when the signals are strongly correlate i.e. when $\Delta \tau<=0.1 T_{c}$. However, [9] shows that in order to CPD-GEVD works at least two factor matrices should be full-rank. Yet, in this work we needed to guarantee that the three factor matrices $\Gamma,\left(\mathbf{C Q}_{\omega}\right)$, and $\mathbf{A}$ were full-rank. Therefore, if we had a rank deficient A the CPD-GEVD would eventually fail. Thus, we should further investigate the CPD-GEVD method in order to identify when and why this decomposition fails even if we guarantee that at least two factor matrices are full-rank

Moreover, herein we overviewed the state-of-the-art tensor based time-delay estimation methods and proposed a new tensor based method. Although, there are several tensor decomposition methods that can be used and may bring further improvements in different scenarios. Therefore, we should investigate new tensor based decomposition and compare it against the methods shown in this thesis. Furthermore, we should create and perform various simulations using different scenarios. 


\section{Bibliography}

[1] K. Uchida, N. Arai, K. Moriya, Y. Miyamoto, T. Kakihara, and T. Tokai, "Development of automatic system for monitoring fishing effort in conger-eel tube fishery using radio frequency identification and global positioning system," Fisheries Science, vol. 71, no. 5, pp. 992-1002, 2005.

[2] M. Antonini, M. Ruggieri, R. Prasad, U. Guida, and G. F. Corini, "Vehicular remote tolling services using EGNOS," IEEE Aerospace and Electronic Systems Magazine, vol. 20 , no. 10 , pp. 3-8, 2005.

[3] C. Kang, S.-H. Lee, and C. C. Chung, "On-road vehicle localization with GPS under long term failure of a vision sensor," in Intelligent Transportation Systems (ITSC), 2015 IEEE 18th International Conference on. IEEE, 2015, pp. 1638-1643.

[4] T. Esau, Q. Zaman, D. Groulx, K. Corscadden, Y. Chang, A. Schumann, and P. Havard, "Economic analysis for smart sprayer application in wild blueberry fields," Precision Agriculture, vol. 17, no. 6, pp. 753-765, 2016.

[5] F. Macchi, "Development and testing of an L1 combined GPS-Galileo software receiver," Ph.D. dissertation, University of Calgary, 2010. [Online]. Available: http://www.geomatics.ucalgary.ca/graduatetheses

[6] M. R. Zanatta, R. K. Miranda, J. P. C. L. da Costa, F. Antreich, and D. V. de Lima, “Antenna array based receivers for third generation global positioning system," in Workshop on Communication Networks and Power Systems, November 2017.

[7] B. Hammoud, F. Antreich, J. A. Nossek, J. A. Nossek, J. P. C. L. d. Costa, and A. L. F. d. Almeida, "Tensor-based approach for time-delay estimation," in WSA 2016; 20th International ITG Workshop on Smart Antennas, March 2016, pp. 1-7.

[8] J. Selva Vera, "Efficient multipath mitigation in navigation systems," Ph.D. dissertation, Universitat Politecnica de Catalunya, 2003.

[9] I. Domanov and L. D. Lathauwer, "Canonical polyadic decomposition of third-order tensors: Reduction to generalized eigenvalue decomposition," SIAM Journal on Matrix Analysis and Applications, vol. 35, no. 2, pp. 636-660, 2014. 
[10] S. U. Pillai and B. H. Kwon, "Forward/backward spatial smoothing techniques for coherent signal identification," IEEE Transactions on Acoustics, Speech, and Signal Processing, vol. 37 , no. 1 , pp. 8-15, 1989.

[11] M. Haardt, F. Roemer, and G. Del Galdo, "Higher-order SVD-based subspace estimation to improve the parameter estimation accuracy in multidimensional harmonic retrieval problems," IEEE Transactions on Signal Processing, vol. 56, no. 7, pp. 31983213, 2008.

[12] T.-J. Shan, M. Wax, and T. Kailath, "On spatial smoothing for direction-of-arrival estimation of coherent signals," IEEE Transactions on Acoustics, Speech, and Signal Processing, vol. 33, no. 4, pp. 806-811, 1985.

[13] P. R. Gomes, A. L. de Almeida, J. P. C. da Costa, J. Mota, D. V. de Lima, and G. Del Galdo, "Tensor-based methods for blind spatial signature estimation in multidimensional sensor arrays," International Journal of Antennas and Propagation, vol. $2017,2017$.

[14] D. V. de Lima, J. P. C. L. da Costa, F. Antreich, and G. Del Galdo, "High resolution time-delay estimation via direction of arrival estimation and Khatri-Rao factorization for multipath mitigation," in Workshop on Smart Antennas (WSA), Berlin, 2017.

[15] R. Roy, A. Paulraj, and T. Kailath, "Estimation of signal parameters via rotational invariance techniques - esprit," in Military Communications Conference Communications-Computers: Teamed for the 90's, 1986. MILCOM 1986. IEEE, vol. 3, Oct 1986, pp. 41.6.1-41.6.5.

[16] F. Roemer and M. Haardt, "Tensor-based channel estimation (TENCE) for two-way relaying with multiple antennas and spatial reuse," in 2009 IEEE International Conference on Acoustics, Speech and Signal Processing, April 2009, pp. 3641-3644.

[17] J. P. C. L. da Costa, D. Schulz, F. Roemer, M. Haardt, and J. A. Apolinário, "Robust RD parameter estimation via closed-form PARAFAC in kronecker colored environments," in 2010 7th International Symposium on Wireless Communication Systems, Sept 2010, pp. 115-119.

[18] F. Roemer and M. Haardt, "A closed-form solution for parallel factor (PARAFAC) analysis," in 2008 IEEE International Conference on Acoustics, Speech and Signal Processing, March 2008, pp. 2365-2368.

[19] _ _ "A closed-form solution for multilinear PARAFAC decompositions," in 2008 5th IEEE Sensor Array and Multichannel Signal Processing Workshop, July 2008, pp. 487491.

[20] —-, "A semi-algebraic framework for approximate cp decompositions via simultaneous matrix diagonalizations (secsi)," Signal Processing, vol. 93, no. 9, pp. 
2722 - 2738, 2013. [Online]. Available: http://www.sciencedirect.com/science/article/ pii/S0165168413000704

[21] T. Fu and X. Gao, "Simultaneous diagonalization with similarity transformation for non-defective matrices," in 2006 IEEE International Conference on Acoustics Speech and Signal Processing Proceedings, vol. 4, May 2006, pp. IV-IV.

[22] J.-F. Cardoso and A. Souloumiac, "Jacobi angles for simultaneous diagonalization," SIAM Journal on Matrix Analysis and Applications, vol. 17, no. 1, pp. 161-164, 1996. [Online]. Available: https://doi.org/10.1137/S0895479893259546

[23] D. V. de Lima, J. P. da Costa, F. Antreich, R. K. Miranda, and G. Del Galdo, "TimeDelay estimation via CPD-GEVD applied to tensor-based GNSS arrays with errors," in 2017 IEEE 7th International Workshop on Computational Advances in Multi-Sensor Adaptive Processing (CAMSAP) (IEEE CAMSAP 2017), Curaçao, Netherlands Antilles, Dec. 2017.

[24] J. P. C. L. da Costa, F. Roemer, M. Haardt, and R. T. de Sousa, "Multi-dimensional model order selection," EURASIP Journal on Advances in Signal Processing, vol. 2011, no. 1, p. 26, Jul 2011. [Online]. Available: https://doi.org/10.1186/1687-6180-2011-26

[25] K. Liu, J. P. C. da Costa, H. C. So, L. Huang, and J. Ye, "Detection of number of components in candecomp/parafac models via minimum description length," Digital Signal Processing, vol. 51, pp. 110 - 123, 2016. [Online]. Available: http://www.sciencedirect.com/science/article/pii/S1051200416000063

[26] J. P. C. L. da Costa, Parameter Estimation Techniques for Multi-Dimensional Array Signal Processing. Shaker Verlag, 2010.

[27] M. S. Braasch and A. Van Dierendonck, "GPS receiver architectures and measurements," Proceedings of the IEEE, vol. 87, no. 1, pp. 48-64, 1999.

[28] R. D. Van Nee, "Spread-spectrum code and carrier synchronization errors caused by multipath and interference," IEEE Transactions on Aerospace and Electronic Systems, vol. 29, no. 4, pp. 1359-1365, 1993.

[29] B. W. Parkingson and J. J. Spilker, "Global positioning system: Theory and applications," American Institute of Aeronautics and Astronautics (AIAA), vol. 1, 1996.

[30] G. Siegert, G. Del Galdo, F. Klier, J. Mahr, G. Rohmer, A. Rügamer, and M. Landmann, "Multi-directional over the air testbed for robustness testing of GNSS receivers against jammers and spoofers," in 31st AIAA International Communications Satellite Systems Conference, International Communications Satellite Systems Conferences (ICSSC), 2013. 
[31] A. Konovaltsev, M. Cuntz, C. Haettich, and M. Meurer, "Performance analysis of joint multi-antenna spoofing detection and attitude estimation," in ION International Technical Meeting 2013, January 2013. [Online]. Available: http://elib.dlr.de/81401/

[32] L. D. Lathauwer, B. D. Moor, and J. Vandewalle, "On the best Rank-1 and Rank-(R1 ,R2 ,..,RN) approximation of higher-order tensors," SIAM Journal on Matrix Analysis and Applications, vol. 21, no. 4, pp. 1324-1342, 2000. [Online]. Available: https://doi.org/10.1137/S0895479898346995

[33] Navstar GPS, "Interface specification IS-GPS-800D," U.S. Air Force, Tech. Rep., 2013. [Online]. Available: http://www.gps.gov/technical/icwg/IS-GPS-800D.pdf

[34] J. J. Rushanan, "The spreading and overlay codes for the 11c signal," Navigation, vol. 54, no. 1, pp. 43-51, 2007.

[35] D. V. de Lima, "Multipath mitigation in time-delay estimation via tensor-based techniques for antenna array-based GNSS receivers," Master's thesis, University of Brasilia, March 2017.

[36] L. D. Lathauwer, B. D. Moor, and J. Vandewalle, "A multilinear singular value decomposition," SIAM Journal on Matrix Analysis and Applications, vol. 21, no. 4, pp. 1253-1278, 2000. [Online]. Available: https://doi.org/10.1137/S0895479896305696

[37] G. H. Golub and C. F. Van Loan, Matrix computations. JHU Press, 2012, vol. 3.

[38] M. Weis, G. Del Galdo, and M. Haardt, "A correlation tensor-based model for time variant frequency selective mimo channels," in Proceedings of International ITG/IEEE Workshop on Smart Antennas (WSA'07), 2007. 


\section{Appendix A}

\section{Tensor Calculus}

\section{A.1 Notation}

Scalar are represented by italic letters $(a, b, A, B)$, vector by lowercase bold letters (a, b), matrices by uppercase bold letters $(\mathrm{A}, \mathrm{B})$, and tensors by uppercase bold calligraphic letters $(\mathcal{A}, \mathcal{B})$.

The superscripts ${ }^{\mathrm{T}},{ }^{*},{ }^{\mathrm{H}},{ }^{-1}$, and ${ }^{+}$denote the transpose, conjugate, conjugate transpose (Hermitian), inverse of a matrix, and pseudo-inverse of a matrix, respectively.

For a matrix $\mathbf{A} \in \mathbb{C}^{M \times N}$, the element in the $m$-th row and $n$-th column is denoted by $a_{m, n}$, its $m$-th row is denoted by $(\mathbf{A})_{m,}$, and its $n$-th column is denoted by $(\mathbf{A})_{, n}$. The 2-norm of a matrix $\mathbf{A}$ is denoted by $\|\mathbf{A}\|_{2}$.

For a matrix $\mathbf{A} \in \mathbb{C}^{M \times N}$ with $M<N$, the $\operatorname{diag}\{\cdot\}$ operator extracts the diagonal:

$$
\operatorname{diag}\{\mathbf{A}\} \triangleq\left[\begin{array}{c}
a_{1,1} \\
a_{2,2} \\
\vdots \\
a_{M, M}
\end{array}\right]
$$

The $n$-th mode unfolding of tensor $\mathcal{A}$ is denoted as $[\mathcal{A}]_{(n)}$. The $n$-mode product between tensor $\mathcal{A}$ and a matrix $\mathrm{B}$ is represented as $\mathcal{A} \times{ }_{n}$ B. The $N$-th order identity tensor of size $L \times \cdots \times L$ is denoted by $\mathcal{I}_{N, L}$.

For two $N$-th order tensor $\mathcal{A}$ and $\mathcal{B}$, both of size $I_{1} \times I_{2} \times \cdots \times I_{N}$, composed of individual scalar elementes $a_{i_{1}, i_{2}, \ldots, i_{N}}$ and $b_{i_{1}, i_{2}, \ldots, i_{N}}$, respectively, its inner product is denoted 
by $\langle\mathcal{A}, \mathcal{B}\rangle$, and is defined as

$$
\langle\mathcal{A}, \mathcal{B}\rangle \triangleq \sum_{i_{1}=1}^{I_{1}} \sum_{i_{2}=1}^{I_{2}} \cdots \sum_{i_{N}=1}^{I_{N}} a_{i_{1}, i_{2}, \ldots, i_{N}} b_{i_{1}, i_{2}, \ldots, i_{N}}
$$

The norm of a tensor $\mathcal{A}$, denoted by $\|\mathcal{A}\|_{F}$, is the Frobenius norm described as

$$
\|\mathcal{A}\|_{F} \triangleq \sqrt{\langle\mathcal{A}, \mathcal{A}\rangle}
$$

\section{A.2 Matrix Calculus}

\section{A.2.1 Kronecker product}

Given two matrices $\mathbf{A} \in \mathbb{C}^{I \times J}$ and $\mathbf{B} \in \mathbb{C}^{K \times L}$ their Kronecker product, denote by $\otimes$, is denote as:

$$
\mathbf{A} \otimes \mathbf{B} \triangleq\left[\begin{array}{ccc}
a_{1,1} \mathbf{B} & \cdots & a_{1, J} \mathbf{B} \\
\vdots & \ddots & \vdots \\
a_{I, 1} \mathbf{B} & \cdots & a_{I, J} \mathbf{B}
\end{array}\right] \in \mathbb{C}^{I K \times J L}
$$

\section{A.2.2 Khatri-Rao product}

Given two matrices $\mathbf{A} \in \mathbb{C}^{I \times R}$ and $\mathbf{B} \in \mathbb{C}^{K \times R}$ their Khatri-Rao product, denote by $\diamond$, is denote as:

$$
\mathbf{A} \diamond \mathbf{B} \triangleq\left[(\mathbf{A})_{\cdot, 1} \otimes(\mathbf{B})_{\cdot, 1} \cdots(\mathbf{A})_{\cdot, R} \otimes(\mathbf{B})_{\cdot, R}\right] \in \mathbb{C}^{I J \times R}
$$




\section{A.2.3 Outer product}

The outer product is a special case of the Kronecker product where the outer product of two vectors $\mathbf{a} \in \mathbb{C}^{I}$ and $\mathbf{b} \in \mathbb{C}^{J}$ results in a matrix $\mathbf{C} \in \mathbb{C}^{I \times J}$

$$
\begin{aligned}
\mathbf{a} \circ \mathbf{b} & =\mathbf{a b}^{\mathrm{T}}=\left[\begin{array}{c}
a_{1} \\
\vdots \\
a_{I}
\end{array}\right]\left[\begin{array}{lll}
b_{1} & \cdots & b_{J}
\end{array}\right] \\
& =\left[\begin{array}{ccc}
a_{1} b_{1} & \cdots & a_{1} b_{J} \\
\vdots & \ddots & \vdots \\
a_{I} b_{1} & \cdots & a_{I} b_{J}
\end{array}\right]=\mathbf{C} \in \mathbb{C}^{I \times J},
\end{aligned}
$$

thus the elements of $\mathbf{C}$ satisfy $c_{i j}=a_{i} b_{j}, i \in 1, \ldots, I, j \in 1, \ldots, J$.

Once the outer product can be extended into other dimensions, an outer product of three vectors $\mathbf{a} \in \mathbb{C}^{I}, \mathbf{b} \in \mathbb{C}^{J}$, and $\mathbf{c} \in \mathbb{C}^{K}$ results in a third-order tensor $\mathcal{X} \in \mathbb{C}^{I \times J \times K}$

$$
\mathbf{a} \circ \mathbf{b} \circ \mathbf{c}=\mathcal{X}
$$

$$
\text { and } x_{i j k}=a_{i} b_{j} c_{k}, i \in 1, \ldots, I, j \in 1, \ldots, J, k \in 1, \ldots, K \text {. }
$$

\section{A.2.4 The vec $\{\cdot\}$ operator}

The vec $\{\cdot\}$ operator reshapes a matrix into a vector in such a manner that its vectors are stacked. For instance, for a matrix $\mathbf{A} \in \mathbb{C}^{M \times N}$

$$
\begin{aligned}
\operatorname{vec}\{\mathbf{A}\} & =\operatorname{vec}\left\{\left[\begin{array}{lll}
\mathbf{A}_{1} & \cdots & \mathbf{A}_{N}
\end{array}\right]\right\} \\
& =\left[\begin{array}{c}
\mathbf{A}_{1} \\
\vdots \\
\mathbf{A}_{N}
\end{array}\right] \in \mathbb{C}^{M N} .
\end{aligned}
$$

An importante property of $\operatorname{vec}\{\cdot\}$ operator is that for $\mathbf{X}=\mathbf{A B C}$ with $\mathbf{A} \in \mathbb{C}^{I \times J}$, a diagonal matrix $\mathbf{B} \in \mathbb{C}^{J \times J}$, and $\mathbf{C} \in \mathbb{C}^{J \times K}$

$$
\begin{aligned}
\operatorname{vec}\{\mathbf{X}\} & =\operatorname{vec}\{\mathbf{A B C}\} \\
& =\left(\mathbf{C}^{\mathrm{T}} \diamond \mathbf{A}\right) \operatorname{diag}\{\mathbf{B}\} \in \mathbb{C}^{I K}
\end{aligned}
$$




\section{A.2.5 The unvec $\{\cdot\}$ operator}

The unvec operator reshapes a vector into a matrix of determined size. Thus, for a vector $\mathbf{a}=\left[\mathbf{a}_{1}^{\mathrm{T}}, \ldots, \mathbf{a}_{N}^{\mathrm{T}}\right] \in \mathbb{C}^{M N}$

$$
\begin{aligned}
\underset{M \times N}{\operatorname{unvec}}\{\mathbf{a}\} & =\underset{M \times N}{\operatorname{unvec}}\left\{\left[\begin{array}{c}
\mathbf{a}_{1} \\
\vdots \\
\mathbf{a}_{N}
\end{array}\right]\right\} \\
& =\left[\begin{array}{lll}
\mathbf{a}_{1} & \cdots & \mathbf{a}_{N}
\end{array}\right]
\end{aligned}
$$

\section{A.3 Tensor Calculus}

\section{A.3.1 Tensors}

Vectors are generalizations of scalars, and matrices are generalizations of vectors, tensors are generalizations of matrices. However, matrices are limited to two dimensions while tensors can have any number of dimensions. Therefore, we apply the terms scalar, vector and matrix to 0-, 1-, and 2-dimensional structures. Thus, we reserve the term tensor to structures with 3 or more dimensions.

In (A.11) we examplify a scalar $I \in \mathbb{C}$, a vector $\mathbf{i} \in \mathbb{C}^{3}$, and an identity matrix $\mathbf{I} \in \mathbb{C}^{3 \times 3}$

$$
I=1, \quad \mathbf{i}=\left[\begin{array}{l}
1 \\
0 \\
1
\end{array}\right], \quad \mathbf{I}=\left[\begin{array}{lll}
1 & 0 & 1 \\
0 & 1 & 0 \\
1 & 0 & 1
\end{array}\right]
$$

while in (A.12) a third order tensor $\mathcal{I}_{3,3} \in \mathbb{C}^{3 \times 3 \times 3}$

$$
\begin{aligned}
\mathcal{I}_{3,3}= & {\left[\begin{array}{lll}
0 & 0 & 0 \\
0 & 0 & 0 \\
0 & 0 & 1
\end{array}\right] } \\
& {\left[\begin{array}{lll}
0 & 0 & 0 \\
0 & 1 & 0 \\
0 & 0 & 0 \\
0 & 0 & 0 \\
0 & 0 & 0
\end{array}\right] }
\end{aligned}
$$

Once higher-order tensors can be created, visualization becomes difficult. A $N$ dimensional tensor $\mathcal{A} \in \mathbb{C}^{I_{1} \times I_{2} \times \cdots \times I_{N}}$ can be seen in "slices" by maintaining its first two 
indexes fixed while varying the remaining $N-2$ indexes. For instance, by changing the third index of the third-order identity tensor from (A.12) while fixing the first and second indexes we have

$$
\left(\mathcal{I}_{3,3}\right)_{,,,, 1}=\left[\begin{array}{lll}
1 & 0 & 0 \\
0 & 0 & 0 \\
0 & 0 & 0
\end{array}\right],\left(\mathcal{I}_{3,3}\right)_{,,, 2}=\left[\begin{array}{lll}
0 & 0 & 0 \\
0 & 1 & 0 \\
0 & 0 & 0
\end{array}\right],\left(\mathcal{I}_{3,3}\right)_{\cdot,,, 3}=\left[\begin{array}{lll}
0 & 0 & 0 \\
0 & 0 & 0 \\
0 & 0 & 1
\end{array}\right]
$$

\section{A.3.2 $n$-mode unfolding}

Once tensor representation can be complex to understand, the $n$-mode unfolding provides a way to represent a tensor as a matrix. This is done by fixing the $n$-th index while varying the other indexes in reverse order, concatenating these vectors along the $n+1$-th dimension, then permuting the order of the dimensions from the $n$-th to the $n-1$-th dimensions. For instance, for a third-order tensor $\mathcal{A} \in \mathbb{C}^{2 \times 2 \times \times 2}$ we can write

$$
\mathcal{A}=\frac{\left[\begin{array}{ll}
5 & 6 \\
7 & 8
\end{array}\right]}{\left[\begin{array}{ll}
1 & 2 \\
3 & 4
\end{array}\right]}
$$

then $\mathcal{A}$ has the following unfoldings:

$$
\begin{aligned}
& {[\mathcal{A}]_{(1)}=\left[\begin{array}{llll}
1 & 5 & 2 & 6 \\
3 & 7 & 4 & 8
\end{array}\right],} \\
& {[\mathcal{A}]_{(2)}=\left[\begin{array}{llll}
1 & 3 & 5 & 7 \\
2 & 4 & 6 & 8
\end{array}\right],} \\
& {[\mathcal{A}]_{(3)}=\left[\begin{array}{llll}
1 & 2 & 3 & 4 \\
5 & 6 & 7 & 8
\end{array}\right] .}
\end{aligned}
$$

For a $N$-dimensional tensor, $\mathcal{A} \in \mathbb{C}^{I_{1} \times \cdots \times I_{N}}$, its $n$-mode unfolding, $[\mathcal{A}]_{(n)}$, will be of size $I_{n} \times \prod_{r \neq n} I_{r}$.

\section{A.3.3 $n$-mode product}

The $n$-mode product permits the calculation of the product of a matrix and a tensor by utilizing the $n$-mode unfolding. For instance, for an $N$-dimensional tensor $\mathcal{A} \in$ $\mathbb{C}^{I_{1} \times \cdots \times I_{n} \times \cdots \times I_{N}}$ and a matrix $\mathbb{B} \in \mathbb{C}^{M \times I_{n}}$, the $n$-mode product is then denoted as $\mathcal{A} \times{ }_{n} \mathbf{B}$. 
Therefore, we have the matrix product $\mathrm{B} \cdot[\mathcal{A}]_{(n)}$ folded back into a tensor of size $I_{1} \times \cdots \times M \times \cdots \times I_{N}$.

\section{A.3.4 PARAFAC model}

The PARAFAC model assumes that a given $N$-dimensional tensor $\mathcal{X} \in \mathbb{C}^{I_{1} \times \cdots \times I_{n} \times \cdots \times I_{N}}$ can be decomposed into a summation of a minimum number of rank-one tensors $\mathcal{X}^{(i)}, i=$ $1, \ldots, L$.

$$
\mathcal{X}=\sum_{\ell=1}^{L} \boldsymbol{\mathcal { X }}^{(\ell)}=\sum_{\ell=1}^{L} \mathbf{a}_{\ell}^{(1)} \circ \cdots \circ \mathbf{a}_{\ell}^{(N)}
$$

where $L$ is the model order of the noiseless tensor.

Through defining factor matrices $\mathbf{A}^{(i)}=\left[\mathbf{a}_{1}^{(i)}, \ldots, \mathbf{a}_{1}^{(i)}\right]$, the Equation (A.18) can be denoted in terms of the $n$-mode product of an $N$-dimensional identity tensor $\mathcal{I}_{N, L} \in \mathbb{R}^{L \times \cdots \times L}$ and loading matrices $\mathbf{A}^{(i)}$

$$
\mathcal{X}=\mathcal{I}_{N, L} \times{ }_{1} \mathbf{A}^{(1)} \times{ }_{2} \mathbf{A}^{(2)} \cdots \times{ }_{N} \mathbf{A}^{(N)} .
$$

Moreover, a useful property for a third-order tensor $\mathcal{A}=\mathcal{I}_{N, 3} \times{ }_{1} \mathrm{~A}^{(1)} \times{ }_{2} \mathrm{~A}^{(2)} \times{ }_{3} \mathrm{~A}^{(3)} \in$ $\mathbb{C}^{I_{1} \times I_{2} \times I_{3}}$ with $\mathbf{A}^{(1)} \in \mathbb{C}^{I_{1} \times d}, \mathbf{A}^{(2)} \in \mathbb{C}^{I_{2} \times d}$, and $\mathbf{A}^{(3)} \in \mathbb{C}^{I_{3} \times d}$, its unfolding are:

$$
\begin{aligned}
& {[\mathcal{A}]_{(1)}=\mathbf{A}^{(1)}\left(\mathbf{A}^{(2)} \diamond \mathbf{A}^{(3)}\right)^{\mathrm{T}} \in \mathbb{C}^{I_{1} \times I_{2} I_{3}},} \\
& {[\mathcal{A}]_{(2)}=\mathbf{A}^{(2)}\left(\mathbf{A}^{(3)} \diamond \mathbf{A}^{(1)}\right)^{\mathrm{T}} \in \mathbb{C}^{I_{2} \times I_{3} I_{1}},} \\
& {[\mathcal{A}]_{(3)}=\mathbf{A}^{(3)}\left(\mathbf{A}^{(1)} \diamond \mathbf{A}^{(2)}\right)^{\mathrm{T}} \in \mathbb{C}^{I_{3} \times I_{1} I_{2}} .}
\end{aligned}
$$

\section{A.3.5 Higher-Order SVD}

The Singular Value Decomposition (SVD) decomposes a given matrix $\mathbf{X} \in \mathbb{C}^{I_{1} \times I_{2}}$ into the following fashion:

$$
\mathbf{X}=\mathbf{U} \boldsymbol{\Sigma} \mathbf{V}^{\mathrm{H}}
$$

where $\mathbf{U} \in \mathbb{C}^{I_{1} \times I_{1}}$ is a unitary matrix holding the left-hand singular vectors and its columns are related to the column space of $\mathrm{X}, \Sigma \in \mathbb{C}^{I_{1} \times I_{2}}$ is a matrix containing the singular values $\sigma_{1}, \sigma_{s}, \ldots, \sigma_{\min \left(I_{1}, I_{2}\right)}$ in its diagonal, and $\mathbf{V} \in \mathbb{C}^{I_{2} \times I_{2}}$ is a unitary matrix holding the righthand singular vectors and its rows are related to the row space of $\mathbf{X}$.

In order to generalize the SVD to an $N$-th order tensor the $n$-mode product previously 
described can be applied

$$
\begin{aligned}
\mathbf{X} & =\mathbf{U} \boldsymbol{\Sigma} \mathbf{V}^{\mathrm{H}} \\
& =\boldsymbol{\Sigma} \times{ }_{1} \mathbf{U} \times{ }_{1} \mathbf{V}^{*} \\
& =\boldsymbol{\Sigma} \times{ }_{1} \mathbf{U}^{(1)} \times_{1} \mathbf{U}^{(2)},
\end{aligned}
$$

where $\mathbf{U}^{(1)}=\mathbf{U}$, and $\mathbf{U}^{(2)}=\mathbf{V}^{*}$. Also, $\boldsymbol{\Sigma}$ have the properties of pseudodiogonality, only its diagonal is non-zero, and ordering, where $\sigma_{1} \geq \sigma_{2} \geq \cdots \geq \sigma_{\min \left(I_{1}, I_{2}\right)} \geq 0$. Therefore, we can generalize the SVD to an $N$-th order tensor $\mathcal{X} \in \mathbb{I}_{\nVdash} \times \mathbb{I}_{\nvdash} \times \cdots \times \mathbb{I}_{\mathbb{N}}$ in terms of an $n$-mode product

$$
\mathcal{X}=\mathcal{R} \times{ }_{1} \mathbf{U}^{(1)} \times_{2} \mathbf{U}^{(2)} \cdots \times_{N} \mathbf{U}^{(N)},
$$

where the tensor $\mathcal{R} \in \mathbb{C}^{I_{1} \times I_{2} \times \cdots \times I_{N}}$ is the core tensor and has the property of allorthogonality, which mean that for two subtensors $\boldsymbol{\mathcal { R }}_{i_{n}=\alpha}$ and $\boldsymbol{\mathcal { R }}_{i_{n}=\beta}$ constructed by keeping the index $i_{n}$ fixed, their inner product $\left\langle\boldsymbol{\mathcal { R }}_{i_{n}=\alpha}, \boldsymbol{\mathcal { R }}_{i_{n}=\beta}\right\rangle=0$ for $\alpha \neq \beta$, and ordering.

Finally, the calculation of the HOSVD can be achieved by finding each unitary left singular vector matrix $\mathbf{U}^{(n)}$ from its respective $n$-mode unfolding by applying the SVD to $[\mathcal{X}]_{(n)}$. Then, the core tensor $\mathcal{R}$ can be computed by applying (A.25)

$$
\mathcal{R}=\mathcal{X} \times{ }_{1} \mathbf{U}^{(1)} \times_{2} \mathbf{U}^{(2)} \cdots \times{ }_{N} \mathbf{U}^{(N)} .
$$

\section{A.3.6 Dual-symmetric tensors}

A $2 N$-th order tensor $\mathcal{X} \in \mathbb{I}_{\nVdash} \times \cdots \times \mathbb{I}_{\mathbb{N}} \times \mathbb{I}_{\mathbb{N}+\nVdash} \times \ldots \mathbb{I}_{\nvdash \mathbb{N}}$ is dual-symmetric if and only if there can be a permutation of indexes $P$, resulting in a tensor $\mathcal{X}_{P}$ which follows the particular PARAFAC Decomposition [16]

$$
\mathcal{X}_{P}=\mathcal{I}_{2 N, L} \times{ }_{1} \mathbf{A}_{1}^{(1)} \cdots \times{ }_{N} \mathbf{A}_{N+1}^{(N)}\left(\mathbf{A}^{(1)}\right)^{*} \cdots \times{ }_{2 N}\left(\mathbf{A}^{(N)}\right)^{*}
$$

The dual-symmetric decomposition is useful in signal processing once every correlation tensor follows this decomposition [38]. In order to harness the relation in (A.27), a particular unfolding known as Hermitian-symmetric unfolding [11] applied to a dual-simmetric tensor. The Hermitian-simmetric unfolding of $\mathcal{X} \in \mathbb{C}^{I_{1} \times \cdots \times I_{2 N}}, \mathbf{X}_{\mathrm{H}}$ is defined as:

$$
\mathbf{X}_{\mathrm{H}}=\operatorname{unvec}_{K \times K}\{\operatorname{vec}\{\boldsymbol{\mathcal { X }}\}\} \in \mathbb{C}^{K \times K},
$$

with $K=I_{1}, \ldots, I_{N}$. Therefore, the unfolding can be represented in terms of its factor 
matrices as:

$$
\mathbf{X}_{\mathbf{H}}=\left(\mathbf{A}^{(N)} \diamond \cdots \diamond \mathbf{A}^{(1)}\right)\left(\mathbf{A}^{(N)} \diamond \cdots \diamond \mathbf{A}^{(N)}\right)^{\mathrm{H}} .
$$




\section{ATTACHMENT}

In the following attachments we describe previous works that the author had done before working with GNSS time-delay estimation. Firstly, the Attachment A briefly describes the Double-Arc positioner and the software created to operate the device. Finally, Attachment B presents our endeavor on mice's brain signal processing. Additionally to the work presented in the Attachment section, we also proposed a MIMO architecture for smartbands communication for postoperative patient care in [REF1]. 


\section{ATTACHMENT A}

\section{The Double-Arc Positioner}

Once the number of unmanned aerial vehicles (UAV), commonly known as drone, has been increasing there is a need to study and develop technologies capable of identifying these devices. Such technologies can be used by public organisms and can be crucial on decision making.

Therefore, the Double-Arc positioner created by the Ilmenau University of Technology (TU Ilmenau) was idealized to analyze radar cross section signals. Thus this device would be used to measure how a given material or object reflect radar signals. The Double-Arc positioner herein mentioned has 4 nodes as shown in Figure A.1. Each node is equipped with a signal transmitter and a servomotor to move the nodes 1, 2, and 3 in a semicircle. Furthermore, the fourth node is placed in the center of the device and is used to move the object on its on axis. Thus, both object and signal transmitters can be moved at same time while the receivers collect the reflected signals. Thus, this device can collect real data of real objects in order to study reflection behavior. Therefore, the Double-Arc may aid researchers on developing more robust and accurate radar cross section processing techniques. Such techniques may be used by a variety of institutions such as military, for monitoring airspace, and airports and power stations, for allowing automation of system capable of taken UAV down. In the case of airports it is crucial to have accurate techniques for identifying UAV once these devices may put aircraft security at risk. 


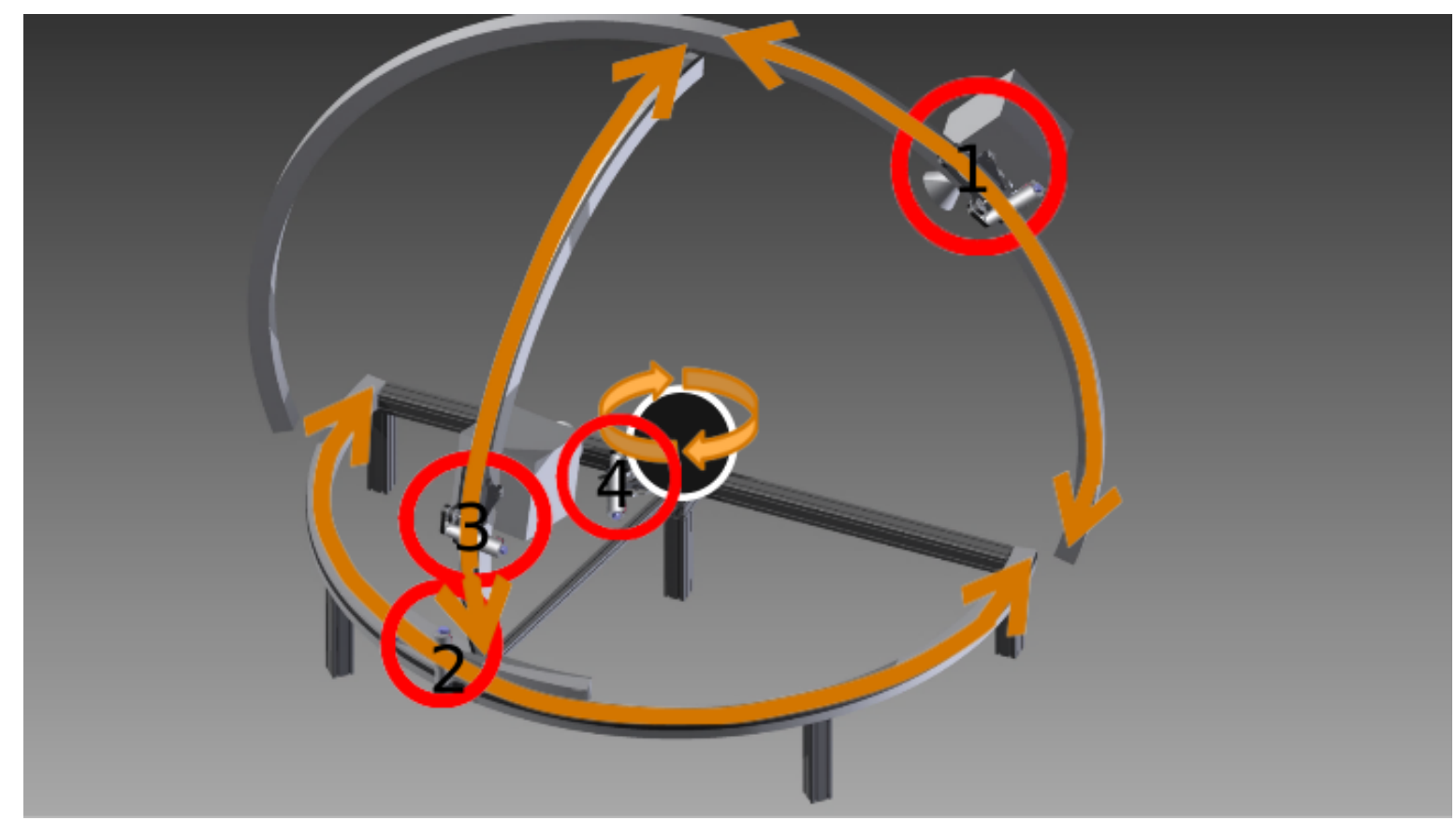

Figure A.1: Double-Arc positioner illustration

Figure A.2 shows the Double-Arc positioner in its final stage with the servomotors attached to it however without the transmitters and the limit switches. These limit switches were used to guarantee that the servomotors do not roam out of bounds. The transmitters would impinge radar signals over the device positioned in node 4.

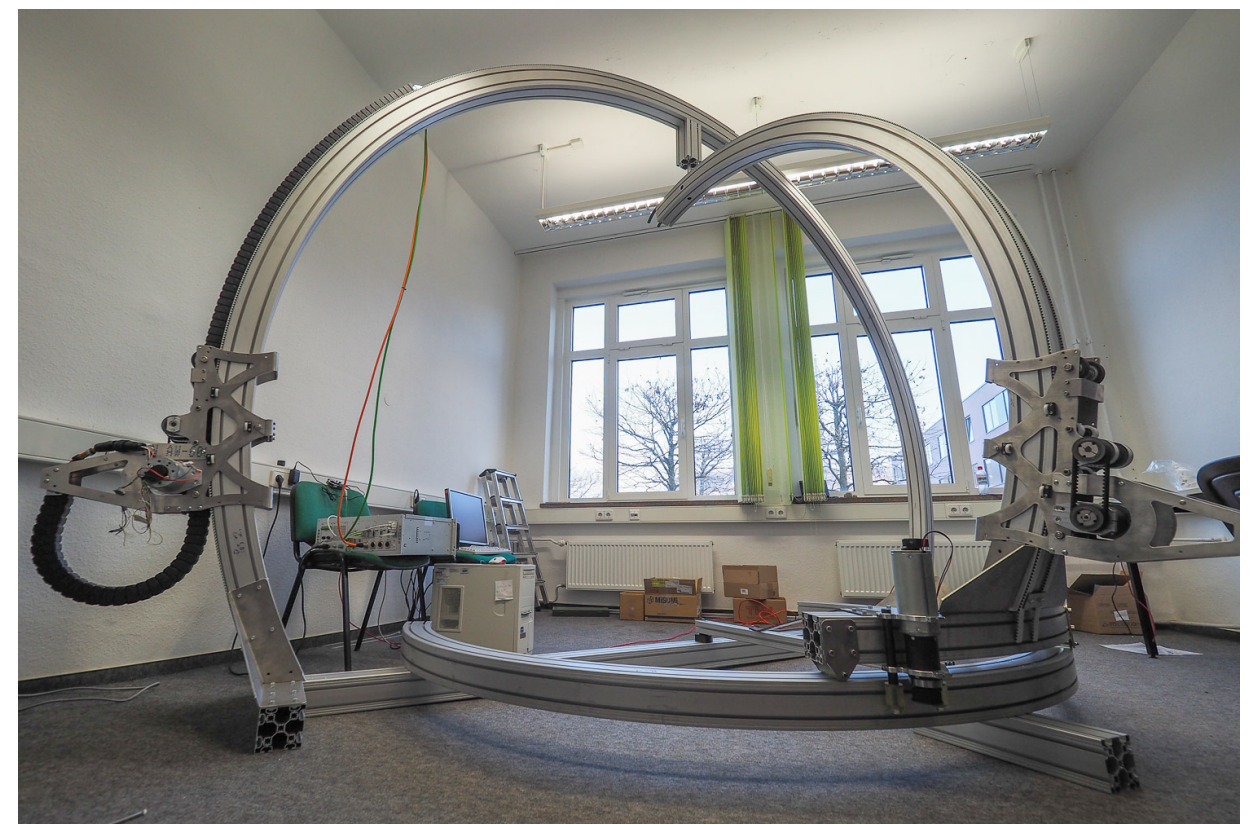

Figure A.2: Double-Arc positioner final stage 


\section{A.1 Software optimization}

Even though the Double-Arc positioner structure was basically done, it was still necessary to develop a software that could control the servomotors. In order to move the servomotors, a C++ code previously developed to a different project was need to be adapted. Additionally, a user interface to send commands to the servomotor was required.

The $\mathrm{C}++$ code was developed to a different project and needed to be embedded to a BeagleBone Black, and adapted to use the CANOpenSocket. The CANOpenSocket is a library that implements the CAN protocol and allows communication through CAN networks in Linux devices. As shown in Figure A.3 various computers should be able to connect to the BeagleBone. Thus, the $\mathrm{C}++$ code generate a SOAP server which allows communication between the software embedded in the BeagleBone Black via TCP/IP and any other device. Then, the BeagleBone receive the messages received through the SOAP server and forward then through the CAN network to the servomotor controller. Finally, the servomotor operates the servomotors by applying the necessary rotation to move the servomotors.

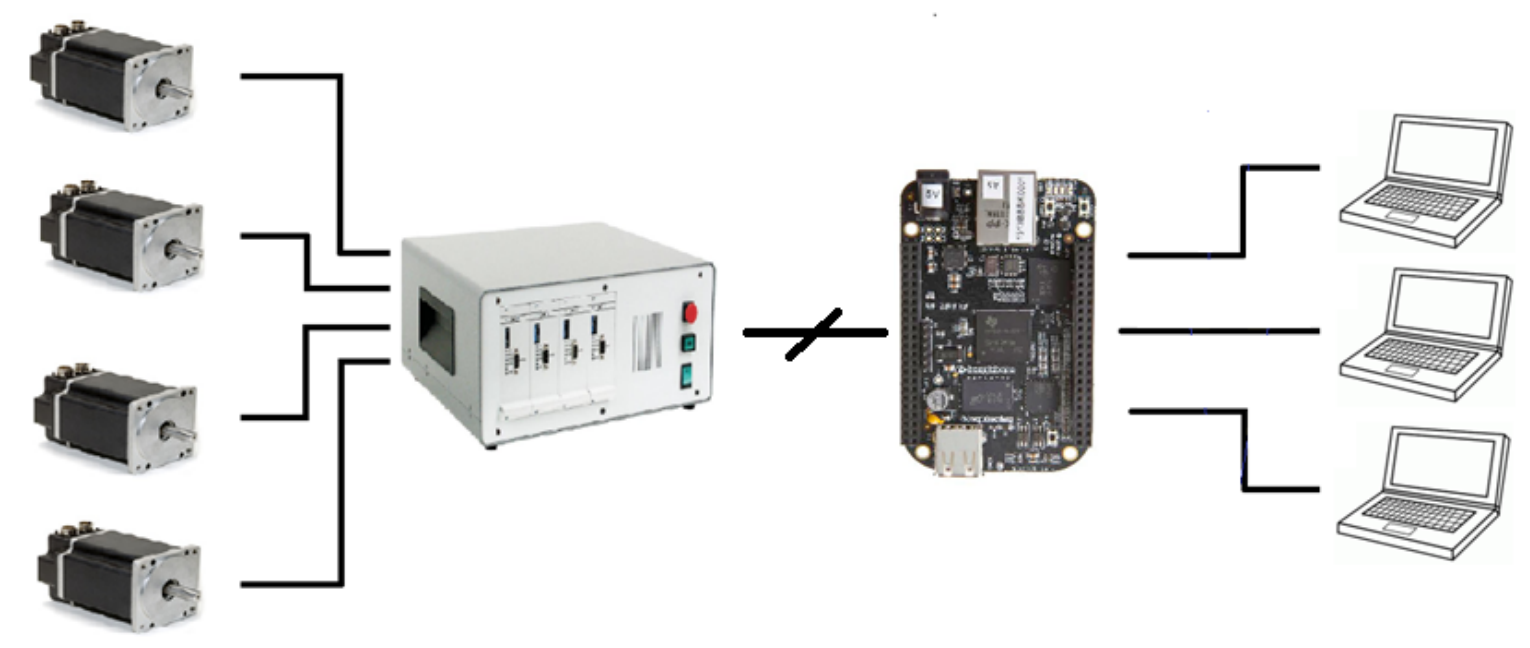

Figure A.3: Double-Arc controller scheme

Once a user interface was required to control the servomotor and all communication were given over the local network, it was firstly developed a PHP code to allow communication between the SOAP server and the user interface. This way, we would allow access to the BeagleBone from anywhere inside the TU Ilmenau network. In order to create the web user interface in in Figure A.4, we used common technologies such HTML, JavaScript and CSS. This interface allowed communication to the BeagleBone by sending commands to the SOAP server. The web user interface implemented simple commands such turn on and shutdown of servomotors. Furthermore, the interface is allowed to send commands to operate the servomotors by sending the position and speed of the servomotor. Therefore, the web user interface simplified command sending procedure by discarding command line interfaces. Besides of sending commands, the interface also displayed the servomotors' actual position 
and the messages sent through the CAN network.

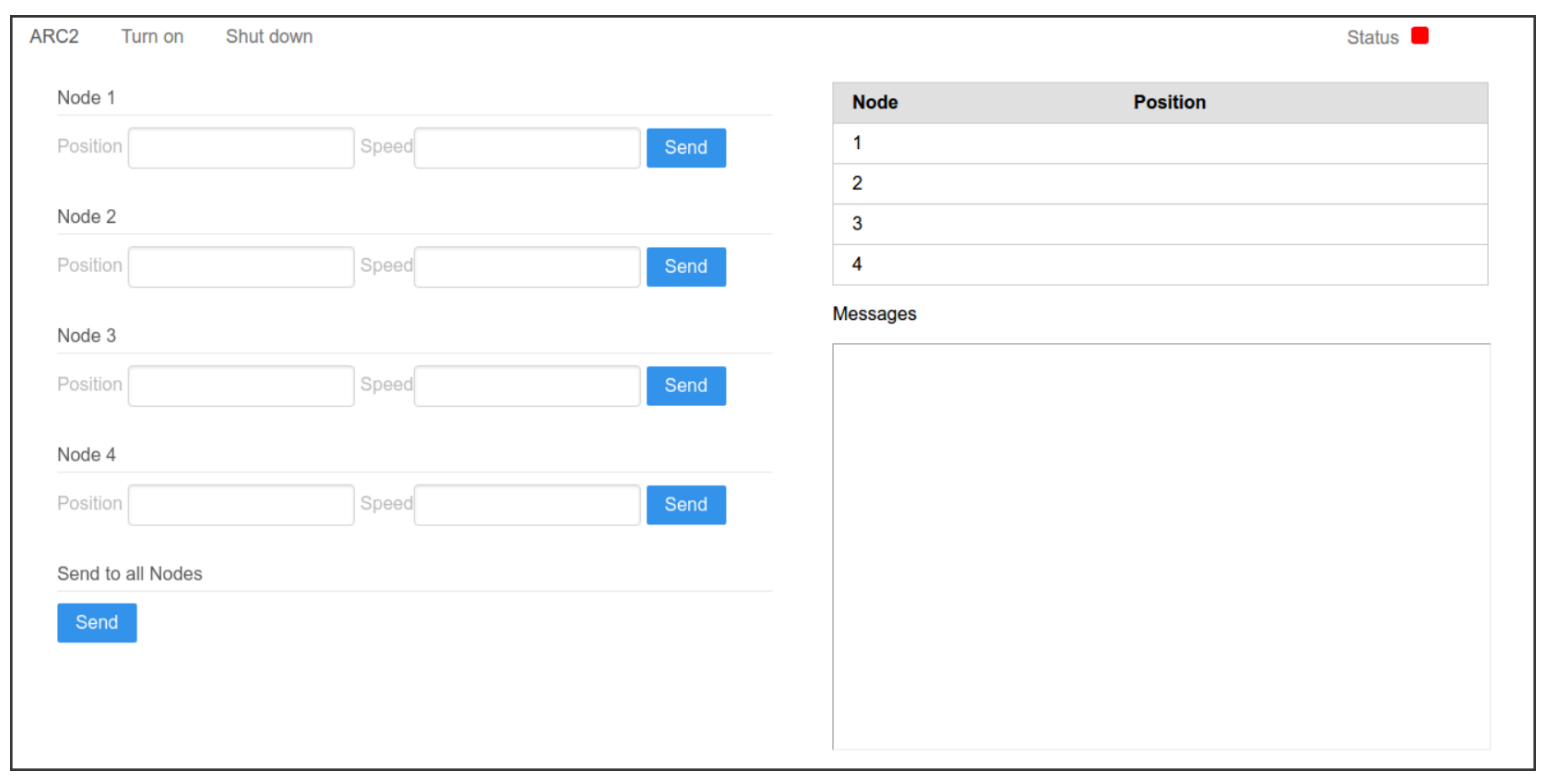

Figure A.4: Double-Arc web user interface 


\section{ATTACHMENT B}

\section{Intracranial Signals}

\section{B.1 Introduction}

By experimenting with animals, scientists can study neural mechanisms and circuits associated with processing and storage of information in the brain. The resulting knowledge arising from such experiments can greatly assist in the treatment of human brain diseases and further be used to understand how brain processes information. Thus, Local Field Potentials (LFP) recordings were introduced in the 1940s [REF2, REF3, REF4]. The LFP are electric potentials recorded from the extracellular space of brain tissue. After its introduction in 1952 [REF5], the Current Source Density (CSD) became a popular method to analyze LFPs [REF6]. Seldomly, Blind Source Separation (BSS) schemes are applied to decompose LFPs or the derived CSDs into their original informative sources. The literature has shown the application of the techniques: Principle Component Analysis (PCA) [REF7], Independent Component Analysis (ICA) [REF8], Spatiotemporal Independent Component Analysis (stICA) [REF9], and more recently the use of a real-valued Parallel Factor Analysis (PARAFAC) approximation [REF10].

With the advent of multilinear electrodes to record intracranial activity, voltage recordings of contacts across cortex laminae can be used to verify which cortex layers are activated by different stimuli. In addition, BSS schemes can support the identification of specific activation groups for given stimuli. Thus, BSS techniques can assist the analysis of such signals. In order to verify the possibility to use multi-way techniques [REF10] proposed an approximation of the real-valued PARAFAC for complex-valued data. In this sense, we propose an improvement in the model created by [REF10] by using Time-Scale Transformation (Wavelets) to study one dimensional cortical recordings. As discussed in [REF11], wavelets have advantages over traditional linear Time-Frequency Analysis (TFA), shuch Short-Time Fourir Transform (STFT), because of its flexibility which allows us to model each wavelet according to signal propoerties. Moreover, neuroeletric signals are highly non-stationary, which makes STFT unsuitable to decompose intracranial signal. This way, we propose to use Continuous Wavelet Transform (CWT) to perform TFA over recorded intracranial 
recordings. Thus, we create an artifial dimension by decomposing the time domain into time and scale, which allows us to perform multi-way analysis once we can stack each time and scale matrix of each electrode.

This paper is organized as follows. In Section B.2, we present biological concepts necessary to understand the origin of the signals recorded by electrodes in the extracellular space of brain tissue. In Section B.3, we show how the data under consideration can be modeled as in the standard BSS problem. In Section B.4, describes Time-Scale Representation (Wavelets) concepts. In Section B.5, we describe the proposed approximation of the realvalued PARAFAC for complex-valued data. In Section B.6, we present the results obtained obtained using the stICA and our PARAFAC, and in Section B.7, the conclusions are drawn.

\section{B.2 Biological Concepts}

Generally in cortical Local Field Potential (LFP) recordings, linearly spaced electrodes are inserted perpendicularly to the cortical surface. Figure B.1 shows the brain area of a mouse studied in this work, the somatosensory cortex.

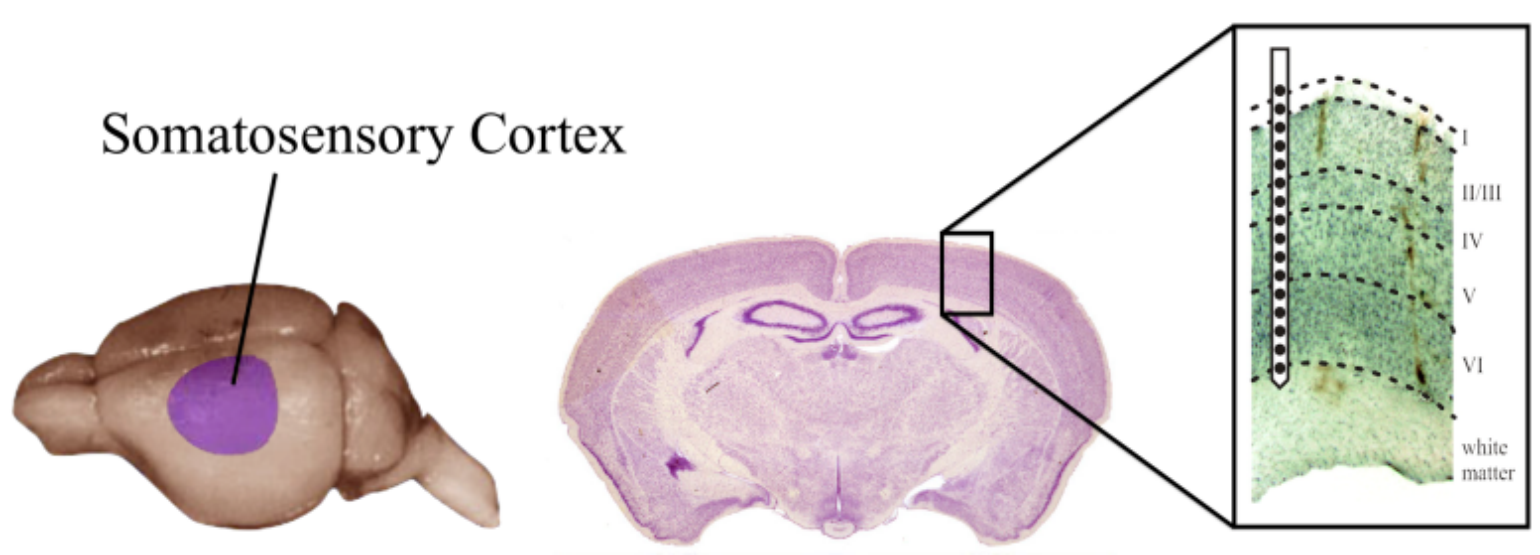

Figure B.1: To the left, perspective view of the brain of a mouse, with the shaded area corresponding to the somatosensory cortex. In the middle, cross section of this same brain. To the right, part of the somatosensory cortex is zoomed, showing a probe with several electrodes inserted for LFP recordings.

The right part of B.1 shows that the neocortex of the mammalian is constituted of six layers, each with specific neuronal population cell bodies. The cells that most contribute to the signal recorded are pyramidal cells [REF12], which receive this name due to their triangular shaped bodies. These cells have elongated projections that receive information from other neurons called apical dendrites, as opposed to other projections with the same function that are not elongated, the basal dendrites. In this sense, the recorded signals represent a superposition of signals originated from individual populations of neurons. Such populations belongs to a similar location and extension.

The Excitatory Postsynaptic Potentials (EPSP) and the Inhibitory Postsynaptic Potentials 
(IPSP) occur along the neurons [REF12, REF13], and the connection between two neurons is the synapse. In the EPSP, positive ions enter the cell through cell membrane in the location of the excitatory synapse, while in IPSP negative ions enter, or positive ions leave, the cell through cell membrane in the location of the inhibitory synapse [REF13]. Therefore, a current sink is created in the location of an excitatory synapse, which is compensated by a distributed current source along the soma dendritic membrane, and a current source is created at the location of an inhibitory synapse, which is compensated by a distributed current sink along the soma dendritic membrane. Since the conventional current flows out the extracellular space in the location of the current sink, which can be thought as positive charge leaving the extracellular space, the extracellular potential at this location becomes more negative. Since the conventional current flows in the extracellular space in the location of the current source, which can be thought as positive charge entering the extracellular space, the extracellular potential at this location becomes more positive. The superposition of similarly distributed currents in different neurons of the same population at the same time results in a higher power signal [REF14].

In the experiment under consideration, our goal is to analyze the evoked potentials (EPs). Since the EPs signals have a very small signal to noise ratio (SNR), several realizations of the same event with stimulus should be repeated and the EPs signals are averaged. For instance, suppose an experimental setting in which a rat received the same sensory stimulus every $\tau$ seconds in a single recording section. Although some criticism can be made, to isolate the evoked potential in one electrode, the continuously recorded data from an electrode has to be subdivided into $\tau_{s}$ epochs. Then, all these windows of the signals are averaged as illustrated in Figure B.2.

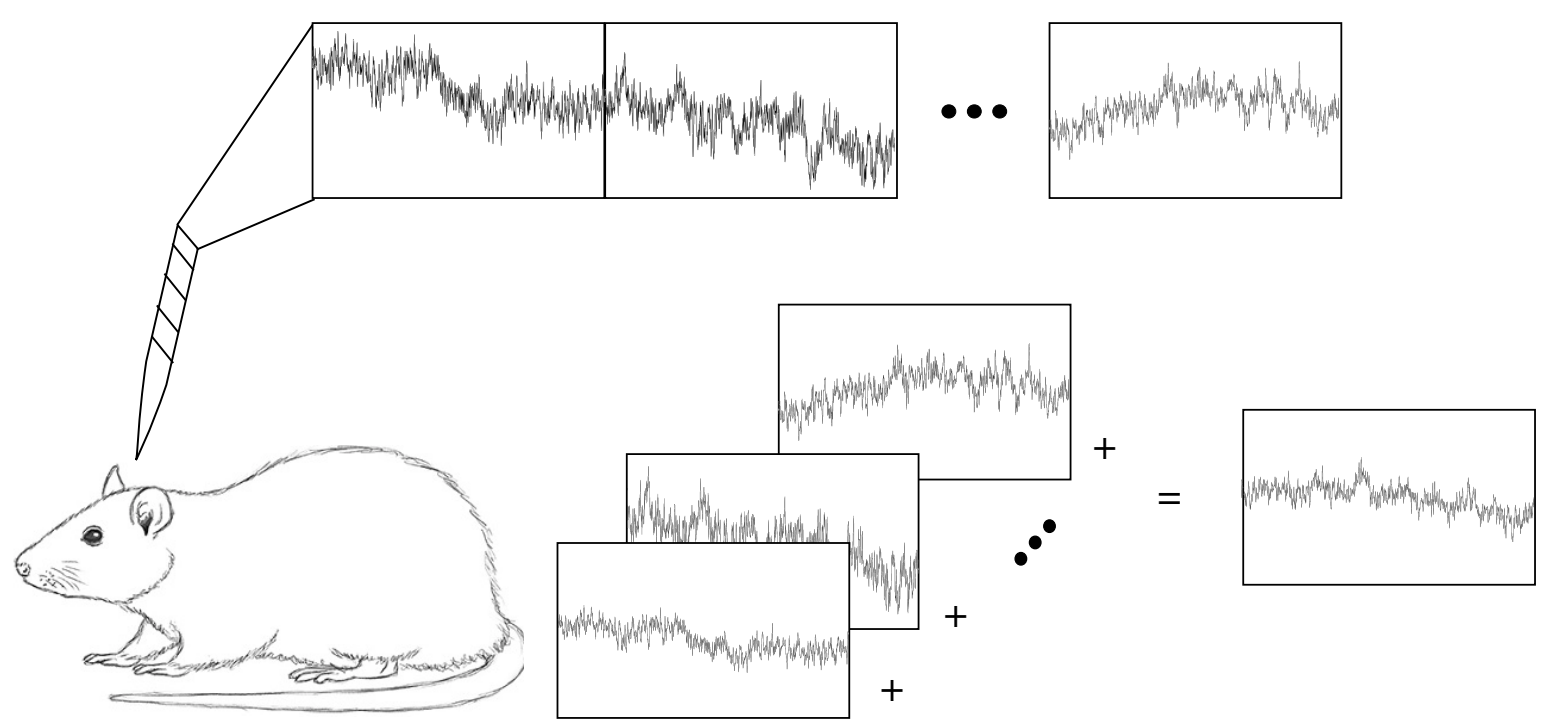

Figure B.2: Experimental setting in which a mouse receives sensory stimulus every few seconds. To isolate the evoked potential in one electrode, one must average over several realizations of the stimulus. This procedure drastically increases the signal to noise ratio compared to a single realization.

In order to estimate the net volume density of current entering or leaving the extracel- 
lular space at different locations, Current Source Density (CSD) analysis is used [REF15]. Consider an arbitrarily small volume of the brain, located at point $(x, y, z)$, where $x, y$ and $z$ are the cartesian coordinates. The net transmembrane current contributed by all cellular elements in this volume, or the Current Source Density (CSD), is related to the extracellular field potential $\phi(x, y, z)$ by the Poisson equation $i(x, y, z)=-\sigma \nabla^{2} \phi(x, y, z)$, where the conductivity $\sigma$ is considered constant and the same in all directions and $\nabla^{2}$ is the laplacian operator [REF16] [REF12]. It is generally assumed that most variation of neural activity is in the vertical direction, due to the laminar structure of the cortex [REF17] [REF12]. Therefore, the CSD can be estimated by LFP recordings from linearly spaced electrodes inserted perpendicularly to the cortical surface as

$$
i(z) \approx-\sigma \frac{\phi(z+\epsilon)-2 \phi(z)+\phi(z-\epsilon)}{\epsilon^{2}}
$$

where $\epsilon$ is the spacing between vertical electrodes.

\section{B.3 Data Model}

For the sake of simplification, current sources and sinks are referred as current sources, with a sink being a source with negative value. As [REF18] described, we neglect inductive effects and, for a fixed time interval, the potential at sensor $p$ at instant $n$ is due to $Q$ groups of synchronized point current sources, called aggregates,

$$
x_{p}(n)=\sum_{q=1}^{Q} h_{p q} s_{q}(n)+v_{p}(n),
$$

where $s_{q}(n)$ is the time course common to the point current sources of the $q$ th aggregate, $h_{p q}$ is a weight that depends on the magnitudes of the point current sources belonging to the $q$ th aggregate and their location with respect to the $p$ th sensor, and $v_{p}(n)$ is the noise at sensor $p$ at instant $n$.

We highlight that the synchronous point current sources of the $q$ th aggregate do not need to be from the same population. According to [REF7], they can be from spatially distinct neuron populations of separate cortical laminae if their transmembrane currents substantially covary over time.

With a change in notation, Equation (B.2) can be rewritten as

$$
x_{p n}=\sum_{q=1}^{Q} h_{p q} s_{q n}+v_{p n},
$$


with $p=1, \ldots, P$ and $n=1, \ldots, N$, which allows for the representation

$$
\mathbf{X}=\sum_{q=1}^{Q} \mathbf{h}_{q} \mathbf{s}_{q}+\mathbf{V},
$$

where $\mathbf{X}$ is a $P \times N$ matrix with elements $x_{p n}, \mathbf{h}_{q}$ is a $P \times 1$ column vector with elements $h_{p q}, \mathbf{s}_{q}$ is a $1 \times N$ row vector with elements $s_{q n}$, and $\mathbf{V}$ is a $P \times N$ matrix with elements $v_{p n}$. This is equivalent to [REF19]

$$
\mathbf{X}=\mathbf{H S}+\mathbf{V}
$$

where $\mathbf{x}_{p}$ are the rows of the $P \times N$ matrix $\mathbf{X}, \mathbf{h}_{q}$ are the columns of the $P \times Q$ matrix $\mathbf{H}$ and $\mathbf{s}_{q}$ are the rows of the $Q \times N$ matrix $\mathbf{S}$. Our objective is to estimate vectors $\mathbf{h}_{q}$ and $\mathbf{s}_{q}$ so that we can plot the CSD figure for each aggregate $q$ separately. In this work, whenever CSD analysis is performed before BSS, it will be considered as a preprocessing step, i.e. high pass filtering of the columns of $\mathbf{X}$.

\section{B.4 Time-Scale Representation}

\section{B.4.1 Continuous Wavelet Transform}

\section{B.5 Proposed technique}

As previously cited, Wavelets can be chosen and modeled according to the signal of interest. Then, hereby, we propose to use the Meyer Wavelet. The Meyer Wavelet is the mother wavelet that better matches EEG signals [REF11]. The Meyer scale function and Meyer wavelet are defined in the frequency domain as shown in Equation $v$ [?]

$$
v(x)=\left\{\begin{array}{lll}
0 & \text { if } & x<0 \\
x & \text { if } & 0 \leq x \leq 1 \\
1 & \text { if } & x>1
\end{array}\right.
$$

Meyer scale function:

$$
\Phi(w)=\left\{\begin{array}{l}
\frac{1}{\sqrt{2 \pi}} \quad \text { if } \quad w \leq \frac{2 \pi}{3} \\
\frac{1}{\sqrt{2 \pi}} \cos \left(\frac{\pi}{2} v\left(\frac{3 w}{2 \pi}-1\right)\right) \quad \text { if } \quad \frac{2 \pi}{3} \leq w \leq \frac{4 \pi}{3} \\
0 \quad \text { otherwise }
\end{array}\right.
$$


The wavelet's spectrum is given as follows

$$
\Psi(w)=\left\{\begin{array}{lll}
\frac{1}{\sqrt{2 \pi}} \sin \left(\frac{\pi}{2} v\left(\frac{3|w|}{2 \pi}-1\right)\right) \exp ^{\frac{2 \pi}{2}} & \text { if } & \frac{2 \pi}{3} \leq|w| \leq \frac{4 \pi}{3} \\
\frac{1}{\sqrt{2 \pi}} \cos \left(\frac{\pi}{2} v\left(\frac{3|w|}{4 \pi}-1\right)\right) \exp ^{\frac{2 \pi}{2}} & \text { if } & \frac{4 \pi}{3} \leq|w| \leq \frac{8 \pi}{3}
\end{array}\right.
$$

Where $\Psi$ is a Meyer wavelet in the frequency domain. In order to trasform Equation (B.8) to time domain, Inverse Fourier Transform should be performed. Thus we have the wavelet in the following fashion.

$$
\psi(t)=2 \int_{\frac{2 \pi}{3}}^{\frac{8 \pi}{3}} \Phi\left(\frac{w}{2}\right) \Phi(w-2 \pi) \cos (w(t-0.5)) d w
$$

In order to model the Meyer wavelet it was defined a scale of size $s=256$ with an effective support approximately between $[-28,28]$. The effective support was defined as follows:

$$
\text { es }=\max \left(\frac{1}{N} \sum_{n=1}^{N}|\mathcal{F}\{S(n)\}|\right),
$$

where $N$ is the number of signals, $\mathcal{F}$ is the Fourier Transform, and $S$ is the signal matrix, and es is the calculated effective support.

Then, after using the modeled Meyer Wavelet to perform the CWT we can rewrite the Equation B. 2 in the following tensor fashion

$$
y_{p i k}=\sum_{q=1}^{Q} \tilde{h}_{p q} d_{i q} g_{k q}+a_{p i k}
$$

where $y_{p i k}$ is the tensor gotten after performing CWT on the signal $X$ at tensor $p$ evaluated at time $i$ and frequency $k, g_{k q}$ is the $q$ th component frequency domain representation, whose amplitude is temporally modulated by the coefficients $d_{i q}$, and whose resulting timefrequency representation is scaled by $\tilde{h}_{p q}$ for sensor $p$. In addition, $a_{p i k}$ is the time-scale representation of the noise at sensor $p$ evaluated at time $i$ and frequency $k$.

Finally, each rank one tensor is then converted back to the time domain by applying the Inverse Continuous Wavelet Transform (ICWT).

\section{B.6 Results}

In the experiment a mouse received the same sensory stimulus every $\tau=7$ seconds while Local Field Potentials were recorded from its somatosensory cortex with 32 electrodes spaced $\epsilon=50$ micrometers from each other perpendicularly to the cortical surface. The sig- 
nals were acquired using a sampling rate of $40 \mathrm{kHz}$ and a referential montage. The proposed approach applied either stICA and PARAFAC after performing CSD analysis as a preprocessing. However, the approach previously proposed in [REF10] considered the voltage time courses from $-0.005 \mathrm{~s}$ to $0.095 \mathrm{~s}$.

In the previsou work [REF10], the Figure B.3, four CSD analyses are depicted. The first one corresponds to the original signals, i.e. without applying any blind source separation approach. The second and third CSD analyses correspond to the first and second components with greatest power obtained via the stICA based on [REF9]. Finally, the fourth CSD analysis corresponds to the sum of the two components obtained via stICA. Yet from the previous work, STFT was applied to obtain a three way complex-valued data composed of the electrodes by time by frequency dimensions. Figure B.4 depicts the original signal, the two components with greatest power and the recovered signal by adding the two components. Note that the results obtained in Figure B.4 are consistent with the ones obtained in Figure B.3 using stICA.

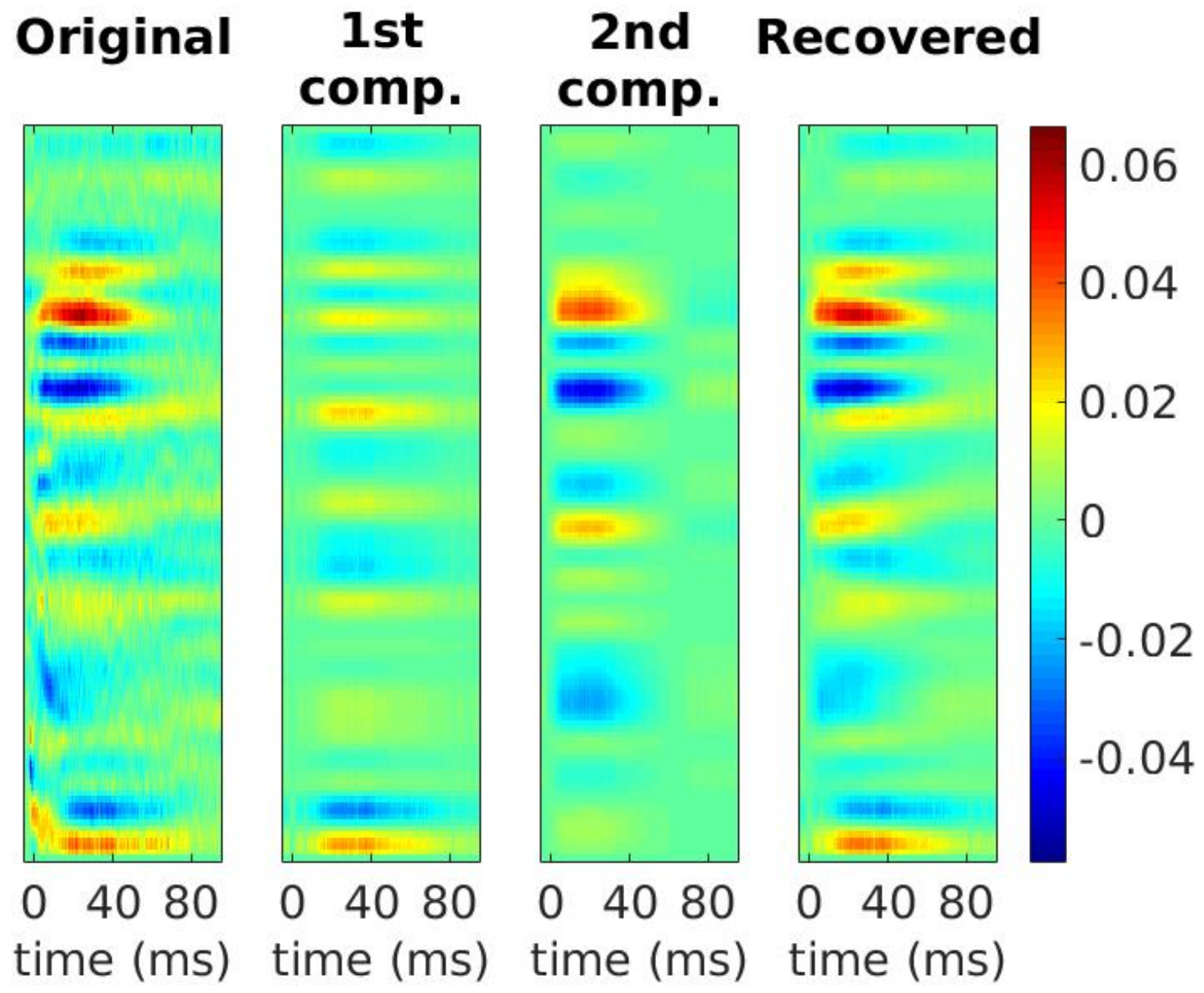

Figure B.3: Four CSD analyses: first CSD analysis of the original measurements, second CSD analysis of the first component with greatest power obtained with stICA, third CSD analysis of the second component with greatest power obtained with stICA, and fourth CSD analysis with the sum of the two components obtained with stICA

Therefore, the herein proposed approach was performed by blindly separating two and 


\section{Original 1st 2nd Recovered}

\section{comp. comp.}
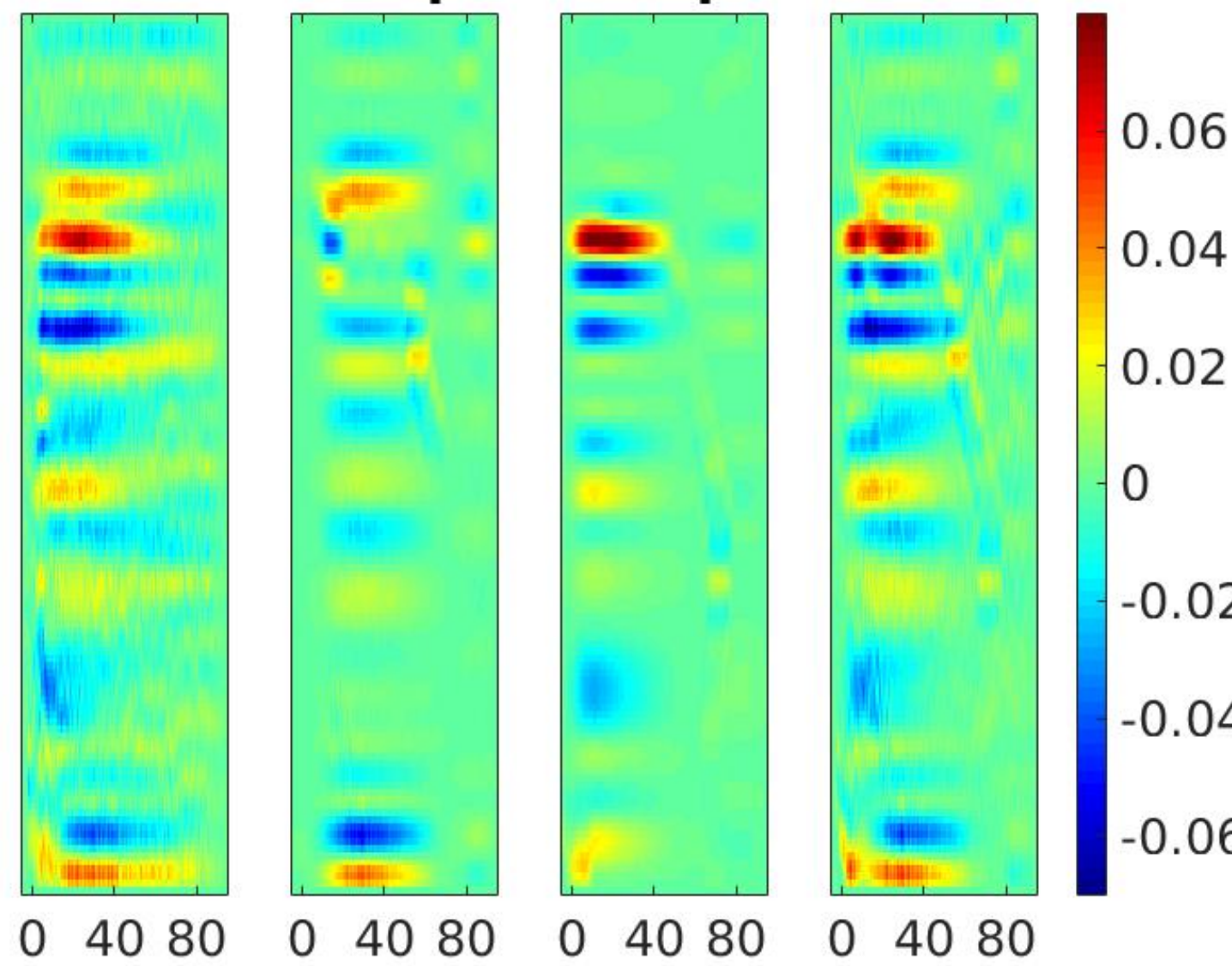

\section{time (ms)}
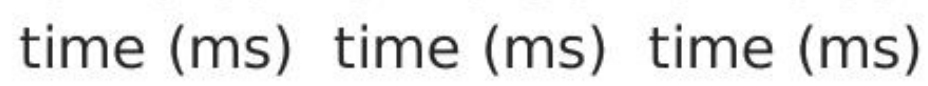

Figure B.4: Four CSD analyses: first CSD analysis of the original measurements, second CSD analysis of the first component with greatest power, third CSD analysis of the second component with greatest power, and fourth CSD analysis with the sum of the two components obtained with real-valued PARAFAC approximation

four components, respectively. Firstly, in Figure B.5 we show four CSD anasyses. The first one depicts the original signal, the second and third CSD analyses correspond to the first and second components with greatest power obtained via the proposed technique. Note that by comparing with Figure B.3 our proposed approach fails to separate the current sources and sinks and also shows a worst performance than the method proposed in [REF10]. Similarly, in Figure B.6, we performed blind separation of four components. The first CSD analyses depicts the original signal, the second, third, fourth and fifth CSD correspond to first, second, third, fourth, and fifth components obtained via the proposed technique. Again, observe that by comparing with Figure B.3 our proposed blind separation method using CWT fails to separate the current sources and sinks. 

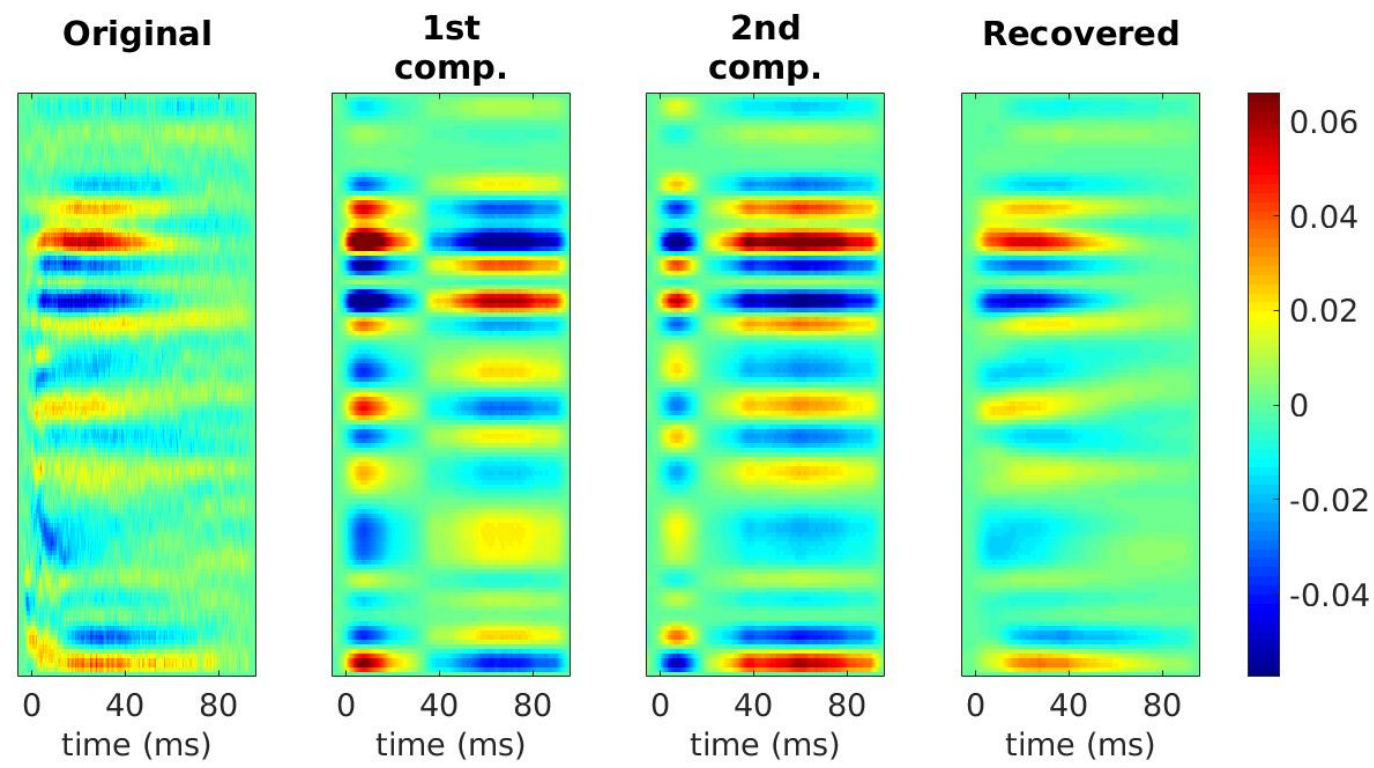

Figure B.5: Four CSD analyses using the proposed approach: first CSD analysis of the original measurements, second CSD analysis of the first component with greatest power, second CSD analysis of the second component with greatest power, and third CSD analysis with the sum of the two components obtained from the real-valued PARAFAC approximation with CWT
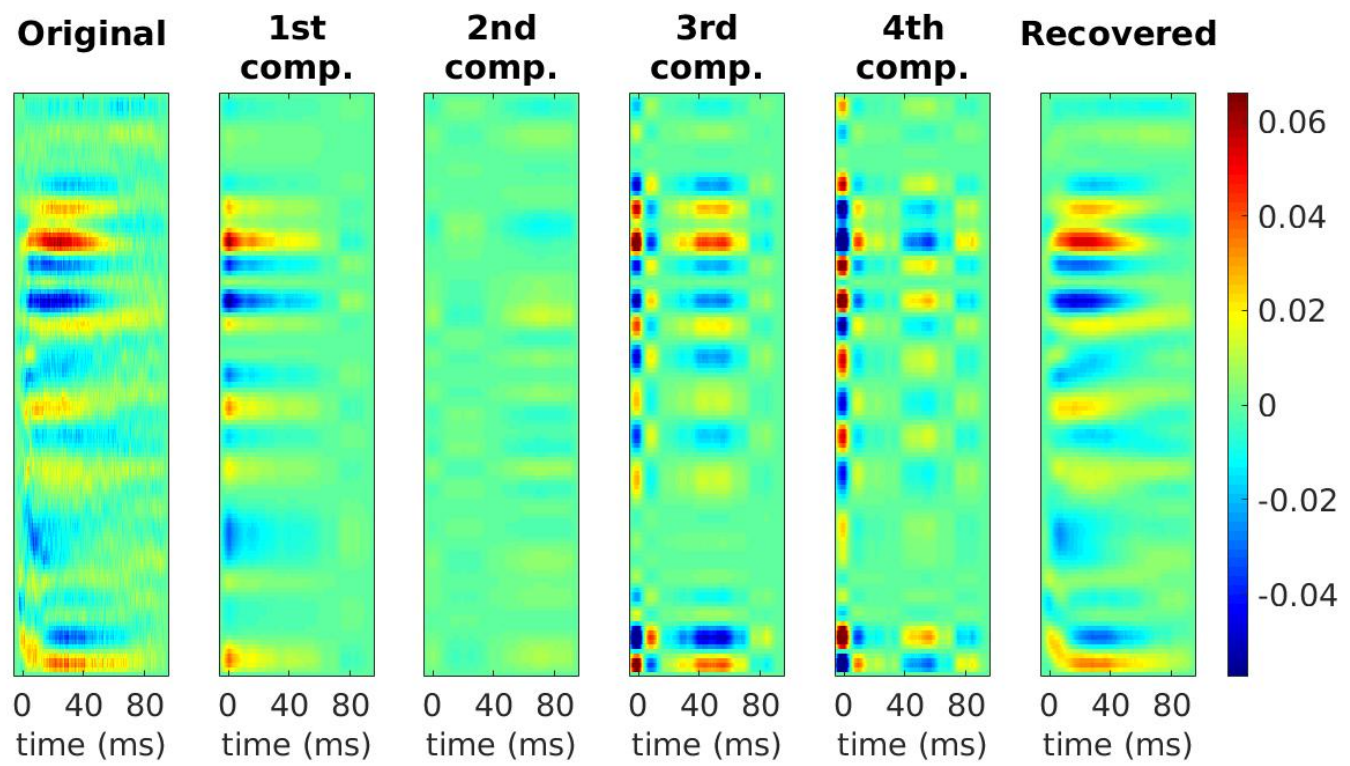

Figure B.6: Six CSD analyses using the proposed approach: first CSD analysis of the original measurements, second CSD analysis of the first component with greatest power, second CSD analysis of the second component with greatest power, third CSD analysis of the second component with greatest power, fourth CSD analysis of the second component with greatest power, fifth CSD analysis of the second component with greatest power, and sixth CSD analysis with the sum of the two components obtained from the real-valued PARAFAC approximation with CWT 


\section{B.7 Conclusion}

In this work, we propose the analysis of the somatosensory cortex signals of a mouse by applying BSS schemes. We also propose the use of multi-way analysis techniques to study recordings from a one dimensional grid of electrodes. For that, besides electrodes and time, another dimension is necessary. This can be achieved by means of Time-Scale Representation techniques. Thus, we proposed to use the Continuous Wavelet Transform (CWT) in order to create a virtual dimension. The CWT was performed in order to allow the use of the PARAFAC decomposition into the measured data. However, through performing blind separation with different numbers of extracted components, we show that the CWT approach herein proposed is not compotible with the used PARAFAC decomposition approach. 


\section{References}

[REF1] J. B. Prettz, J. P. C. L. da Costa, J. R. Alvim, R. K. Miranda, and M. R. Zanatta, "Efficient and low cost mimo communication architecture for smartbands applied to postoperative patient care," in 2017 Second Russia and Pacific Conference on Computer Technology and Applications (RPC), Sept 2017, pp. 1-5.

[REF2] T. H. Bullock, "Problems in the comparative study of brain waves," Yale J Biol Med, vol. 17, no. 5, pp. 657-680.3, 1945.

[REF3] R. Galambos, "Cochlear potentials from the bat," Science, vol. 93, p. 215, 1941.

[REF4] W. H. Marshall, C. N. Woolsey, and P. Bard, "Cortical representation of tactile sensibility as indicated by cortical potentials," Science, vol. 85, pp. 388-390, 1937.

[REF5] W. H. Pitts, "Investigations on synaptic transmission," in Cybernetics, Trans. 9th Conf. Josiah Macy Foundation H. von Foerster, New York, 1952, pp. 159-166.

[REF6] E. Bellistri, J. Aguilar, and L. M. d. J. R. Brotons-Mas, G. Foffani, "Basic properties of somatosensory-evoked responses in the dorsal hippocampus of the rat," $J$ Physiol., vol. 591, no. 10, pp. 2667-2686, 2013.

[REF7] D. S. Barth, S. Di, and C. Baumgartner, "Laminar cortical interactions during epileptic spikes studied with principal component analysis and physiological modeling," Brain Research, vol. 484, no. 1-2, pp. 13-35, 1989.

[REF8] V. A. Makarov, J. Makarova, and O. Herreras, "Disentanglement of local field potential sources by independent component analysis," Journal of Computational Neuroscience, vol. 29, no. 3, pp. 445-457, 2010.

[REF9] S. Leski, E. Kublik, D. A. Swiejkowski, A. Wrobel, and D. Wojcik, "Extracting functional components of neural dynamics with independent component analysis and inverse current source density," Journal of Computational Neuroscience, vol. 29, no. 3, pp. 459-473, 2012.

[REF10] J. P. C. L. da Costa, R. K. Miranda, and M. R. Zanatta, “Tensor based Blind Source Separation for Current Source Density Analysis of Evoked Potentials from Somatosensory Cortex of Mice," in 8th International IEEE EMBS Neural Engineering Conference (NER'17), Shanghai, China, May 2017. 
[REF11] V. J. Samar, A. Bopardikar, R. Rao, and K. Swartz, "Wavelet Analysis of Neuroelectric Waveforms: A Conceptual Tutorial," Brain and Language, vol. 66, no. 1, pp. 7-60, 1999. [Online]. Available: http://linkinghub.elsevier.com/retrieve/ pii/S0093934X98920247

[REF12] G. T. Einevoll, C. Kayser, N. K. Logothetis, and S. Panzeri, "Modeling and analysis of local field potentials for studying the function of cortical circuits," Nature Reviews Neuroscience, vol. 14, no. 11, pp. 770-785, 2013.

[REF13] F. Lopes da Silva, "EEG: Origin and measurement," in EEG - fMRI: Physiological Basis, Technique, and Applications, C. Mulert and L. Lemieux, Eds. Springer Science and Business Media, 2009, pp. 19-38.

[REF14] R. Nieuwenhuys, H. Donkelaar, and C. Nicholson, “The Central Nervous System of Vertebrates, Volume 1," vol. 1, 1998.

[REF15] H. T. Glabska, E. Norheim, A. Devor, A. M. Dale, G. T. Einevoll, and D. K. Wojcik, "Generalized laminar population analysis (gLPA) for interpretation of multielectrode data from cortex," Frontiers in Neuroinformatics, vol. 10, pp. 1-15, 2016.

[REF16] C. Nicholson and J. A. Freeman, "Theory of current source-density analysis and determination of conductivity tensor for anuran cerebellum," Journal of Neurophysiology, vol. 38, no. 2, pp. 356-368, 1975.

[REF17] D. K. Wojcik, "Current source density (CSD) analysis," in Encyclopedia of Computational Neuroscience, D. Jaeger and R. Jung, Eds. New York: Springer, 2014.

[REF18] J. Mocks, "Decomposing event-related potentials: A new topographic components model,” Biological Psychology, vol. 26, no. 1-3, pp. 199-215, June 1988.

[REF19] R. Bro, "Multiway analysis in the food industry," Ph.D. dissertation, Universiteit van Amsterdam, 1998. 